UNIVERSIDAD POLITÉCNICA DE MADRID

ESCUELA TÉCNICA SUPERIOR DE INGENIEROS

AERONÁUTICOS

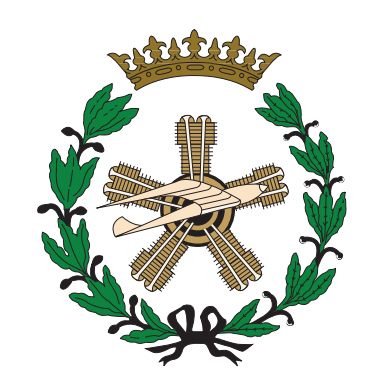

\title{
Adaptive Reduced Order \\ Modeling of Aeroelastic Flows \\ BASED ON Proper ORTHOGONAL \\ DECOMPOSITION
}

\author{
Doctoral Thesis \\ by \\ Rubén Moreno Ramos \\ M.Sc in Aerospace Engineering
}

MADRID, 2017 

DEPARTAMENTO DE MATEMÁTICA APLICADA

A LA INGENIERIA AEROESPACIAL

ESCUELA TÉCNICA SUPERIOR DE INGENIEROS

AERONÁUTICOS

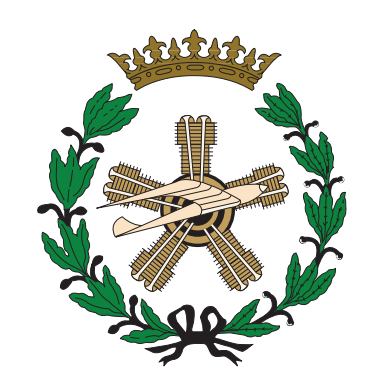

\title{
Adaptive Reduced ORder \\ Modeling of Aeroelastic Flows \\ BASEd on Proper OrThogonal \\ DECOMPOSITION
}

\author{
Doctoral Thesis \\ by \\ Rubén Moreno Ramos \\ M.Sc in Aerospace Engineering \\ Directed by \\ Fernando Varas Mérida José Manuel Vega de Prada \\ Associate Professor \\ Professor
}

MADRID, 2017 



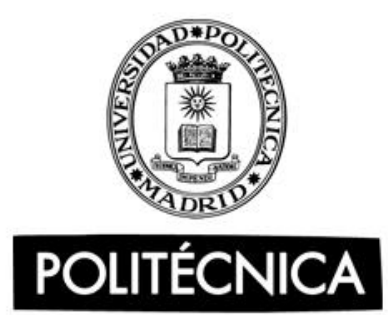

Tribunal nombrado por el Sr. Rector Magfco. de la Universidad Politécnica de Madrid, el día...... de...... de $20 \ldots$.

Presidente:

Vocal:

Vocal:

Vocal:

Secretario:

Suplente:

Suplente:

Realizado el acto de defensa y lectura de la Tesis el día..........de. de $20 \ldots$ en la E.T.S.I. /Facultad.

Calificación

EL PRESIDENTE

LOS VOCALES

EL SECRETARIO 



\section{Abstract}

Aeroelasticity is defined as the science that studies the mutual interaction between the aerodynamic, inertial, and structural forces. Aircraft flexibility has gained nowadays a more prominent role in overall aircraft design due to the continuous search for performance improvements, and weight reduction, supported by new materials, manufacturing techniques and more accurate (and less conservative) stress sizing methods. This has caused that aeroelastic stability and aeroelastic response impose design constraints in the aircraft, in spite of still relying on not so accurate and conservative methods due to the huge complexity of the phenomena. Among the inertial, structural and aerodynamic forces, it is the aerodynamic forces that aeroelasticians have more difficulties to model and simulate accurately. A current industry standard is the use of the doublet lattice model (DLM), which is a linear, unsteady, potential theory based, finite element method for modeling oscillating interfering lifting surfaces in subsonic flows. Since it lacks accuracy, specially in the transonic regime, additional corrections need to be added, based on empirical data or computational fluid dynamics (CFD) analyses, which add uncertainty and hence conservativeness. Several successful applications exist that directly couple a CFD model to the structural model, but the associated computational cost makes it inappropriate for industrial applications as a DLM substitute.

Reduced order models (ROM) based on proper orthogonal decomposition (POD) have been shown to be a very good option to produce CFD equivalent accuracy at a much lower cost. Until now, such ROMs would primarily be able to reproduce data in similar conditions to previously existing simulations, and usually for steady flows, since for unsteady analyses the complexity is not only spatial but also temporal. With the advent of self adaptive POD based ROMs, in which the ROM works in parallel with a full numerical solver, taking over when accuracy is not compromised, good results have been obtained in speeding up time accurate simulations for the Complex-Ginzburg Landau equation.

The work presented in this thesis serves as a proof of concept on the extension of such self adaptive POD based ROMs for unsteady aerodynamic prediction in a computational aeroelasticity framework. The method is first presented and, then, an application to unsteady aerodynamics and aeroelastic simulations is performed for inviscid and viscous turbulent flows. An unsteady CFD solver has been developed in parallel with the $\mathrm{ROM}$ application, since full control of the simulation was required. Additionally, in order to reduce the computational cost while maintaining representativity to industrial environments, the simulations have been performed on an airfoil instead of a lifting surface. The results show very good potential for such simulations and even higher gains are expected when moving to three-dimensional simulations. 


\section{Resumen}

Se define como Aeroelasticidad la ciencia que estudia la interacción entre las fuerzas aerodinámicas, incerciales y elásticas. La flexibilidad de la estructura que conforma un avión gana cada vez más importancia en su diseño global, debido a la contínua búsqueda de mejoras en la eficiencia a través principalmente de la reducción de peso, apoyada por mejoras en las técnicas de fabricación y técnicas de dimensionado estructural menos conservativas. De esta manera, el diseño de los aviones se ve limitado por la respuesta aeroelástica y por inestabilidades a pesar de que, debido a la tremenda complejidad de los fenómenos, su predicción depende de métodos conservativos no demasiado precisos. Las fuerzas aerodinámicas no estacionarias son la principal fuente de incertidumbre. A día de hoy, el estándar en la industria es el uso del modelo de entramado de dobletes (DLM por sus siglas en inglés), que es un método de elementos finitos basado en teoria potencial. Debido a sus limitaciones, especialmente visibles en el régimen transónico, son necesarias correcciones adicionales basadas en datos experimentales o provenientes de simulaciones de dinámica de fluidos computacional (CFD), que añaden incertidumbre y, por lo tanto, conservadurismo. Se ha conseguido con éxito, en varias instancias, acoplar directamente los métodos CFD con el modelo estructural y realizar simulaciones aeroelásticas, pero el elevado coste computacional que requiere lo hace inaplicable en un entorno industrial como sustituto del DLM.

Los modelos de orden reducido ( $R O M$ ) basados en la decomposición ortogonal propia (POD) han demostrado ser una buena opción para producir resultados equivalentes al CFD a un coste mucho menor. Hasta ahora, dichos ROM eran capaces de reproducir soluciones cuyas condiciones no se alejaban mucho de la base de datos preprocesada, principalmente en simluaciones estacionarias debido a que la complejidad temporal se añade a la espacial en el caso no estacionario. Con el advenimiento de los ROM basados en POD, en los cuales el ROM trabaja en paralelo al algoritmo numérico y lo sustituye siempre y cuando la precisión de los resultados no se vean comprometidos, se han obtenido buenos resultados en simulaciones temporales de la ecuación compleja de Ginzburg-Landau.

El trabajo presentado en esta tesis sirve como prueba de concepto para la extensión de dichos $\mathrm{ROM}$ a la predicción de fuerzas aerodinámicas no estacionarias en simulaciones de aeroelasticidad computacional. Se presenta el método en primer lugar para, a continuación, demostrar su aplicación en el cálculo de fuerzas aerodinámicas y en la simulación de respuesta aeroelástica, tanto para flujos no viscosos como viscosos turbulentos. Cómo se desea tener total control sobre el algoritmo numérico, ha sido necesario desarrollar un código CFD en paralelo a la aplicación de los modelos de orden reducido. Adicionalmente, siendo necesario reducir el coste computacional, las simulaciones se han realizado sobre un perfil aerodinámico en lugar de una superficie sustentadora. Los resultados muestran muy buen potencial para dichas simulaciones e incluso se esperan ganancias mayores en casos tridimensionales. 


\section{Agradecimientos}

En primer lugar quiero agradecer a mi mujer Patricia por su apoyo, ánimo y amor, sin ellos no habría sido capaz de terminar. A mi hija Marta por todo el tiempo de juegos que le he robado, espero poder devolvérselos pronto. A mi madre por enseñarme el valor de la constancia y a mi padre por enseñarme a cuestionar lo establecido. A mi hermana por demostrarme el valor del trabajo duro.

También quiero agradecer a mis directores de tesis, José Manuel Vega y Fernando Varas, por su guía, ayuda inestimable y sobre todo por adaptarse a mis complicados, y cambiantes, plazos que me han permitido ser capaz de compaginar la familia, el trabajo y los estudios.

Quiero agradecer también a mi familia y amigos por todo vuestro apoyo. Gracias por entender y suplir mis ausencias en épocas de estudio.

\section{Aknowledgements}

I would like to thank also all the excellent engineers that I am honored to have had as mentors along my professional career. Paul F. Taylor, Héctor Climent, Detlef Schierenbeck, Kai Seyffarth, Félix Arévalo, Miguel Martín-Cifuentes, Mercedes Oliver, Jean Paul Esquerré, and many more I am sure I forget right now, thanks for showing me the way. Thanks for showing me that the better is enemy of the good. 


\section{Contents}

1 Introduction and targets 1

2 Theoretical background $\quad 6$

2.1 Fluid structure interaction . . . . . . . . . . . . . . . . 6

2.2 POD based reduced order model . . . . . . . . . . . . . . . . 7

2.2 .1 POD decomposition . . . . . . . . . . . . . . 7

2.2.2 Flow field as combination of POD modes . . . . . . . . . . 9

2.2 .3 Residual minimization . . . . . . . . . . . . . . . . . . . 9

2.3 Adaptive POD on the fly . . . . . . . . . . . . . . . . . 10

2.3.1 POD based ROM drawbacks . . . . . . . . . . . . . 10

2.3 .2 POD on the fly . . . . . . . . . . . . . . . 10

2.4 Definition of the inner product . . . . . . . . . . . . . 12

2.4.1 Standard inner product $\ell^{2} \ldots \ldots \ldots \ldots \ldots 12$

2.4.2 Standard inner product $L^{2} \ldots \ldots \ldots \ldots \ldots$

2.4.3 Energy based $L_{E}$ inner product . . . . . . . . . . . . . 13

2.4 .4 Selection based inner product . . . . . . . . . . . . . . . 13

3 Computational fluid dynamics solver $\quad \mathbf{1 5}$

3.1 Introduction . . . . . . . . . . . . . . . . . . . . 15

3.2 Governing equations . . . . . . . . . . . . . . . . 15

3.3 Computational domain . . . . . . . . . . . . . . . . 16

3.4 Convective terms . . . . . . . . . . . . . . . . . 16

3.4.1 Divergence based terms . . . . . . . . . . . . . 17

3.4 .2 Gradient terms . . . . . . . . . . . . . . . . . 18

3.4.3 Convection differencing scheme . . . . . . . . . . . . . . 19

3.5 Diffusive terms . . . . . . . . . . . . . . . . . . . 19

3.6 Scaling and non-dimensionalization . . . . . . . . . . . . . . 20

3.7 Boundary conditions . . . . . . . . . . . . . . . . . . . . . 21

3.8 Primitive and conserved variables . . . . . . . . . . . . . . . 24

4 Fluid structure interaction $\quad 25$

4.1 Structural model . . . . . . . . . . . . . . . . . . . . 25

4.1 .1 Lagrangian mechanics . . . . . . . . . . . . . 25 
4.1.2 Example: Airfoil with control surface . . . . . . . . . . 26

4.2 Fluid-structure coupling . . . . . . . . . . . . . . . . . . . . . . 29

4.2 .1 Rigid body . . . . . . . . . . . . . . . . . . . 29

4.2 .2 Deformable body . . . . . . . . . . . . . . . . . . 30

5 Inviscid simulation application $\quad 32$

5.1 Forced oscillation . . . . . . . . . . . . . . . . . . 33

5.1.1 Computational fluid domain model . . . . . . . . . . . . 33

5.1 .2 Sub-mesh selection . . . . . . . . . . . . . . . . . 36

5.1 .3 Oscillatory forcing . . . . . . . . . . . . . . 37

5.1.4 Results for the time dependent subsonic flow at $\mathrm{M}=0.2 \ldots 38$

5.1.5 Results for the time dependent transonic flow at $\mathrm{M}=0.8 \ldots \ldots 4$

5.2 Remarks on solution methods and ROM efficiency . . . . . . . . . . . . 44

5.2 .1 Number of modes . . . . . . . . . . . . . . . . . . . . . 44

5.2.2 Solution method and tunable parameters effects . . . . . . . 45

5.3 Aeroelastic simulation . . . . . . . . . . . . . . . . . . . . 51

5.3 .1 Structural model . . . . . . . . . . . . . . . . . . . . . 51

5.3 .2 Analytical flutter solution . . . . . . . . . . . . . . 52

5.3.3 Results for the subsonic flow at $\mathrm{M}=0.2 \ldots \ldots \ldots \ldots$. . . . . . 54

5.3.4 Results for the transonic flow at $\mathrm{M}=0.8 \ldots \ldots \ldots$

5.4 Remarks on ROM efficiency . . . . . . . . . . . . . . . . . 58

5.4.1 ROM efficiency and number of modes . . . . . . . . . . 58

5.4.2 Solution method and tunable parameters effects . . . . . . . 58

5.5 Conclusions . . . . . . . . . . . . . . . . . . . . 60

6 Turbulent viscous flow simulations $\quad 61$

6.1 Additions to the numerical solver for simulations of turbulent flow . . . 61

6.1.1 Turbulence model . . . . . . . . . . . . . . . . . . . 61

6.1 .2 High order convection scheme . . . . . . . . . . . . 63

6.1 .3 Flow solver algorithm . . . . . . . . . . . . . . 66

6.1 .4 Numerical solver validation . . . . . . . . . . . . . . . 67

6.2 Forced harmonic oscillation of a NACA0012 airfoil . . . . . . . . . . 67

6.2 .1 Computational fluid domain model . . . . . . . . . . . . 67

6.2 .2 Sub-mesh selection . . . . . . . . . . . . . . . . . . 69

6.2.3 ROM solution algorithm in the presence of turbulence model . . 71

6.2 .4 Oscillatory forcing . . . . . . . . . . . . . . 72

6.2.5 Results for the time dependent subsonic flow at $\mathrm{M}=0.3 \ldots 73$

6.2.6 Results for the time dependent transonic flow at $\mathrm{M}=0.8 \ldots 76$

6.2.7 Remarks on the ROM efficiency . . . . . . . . . . . . . . 76

6.3 Aeroelastic simulation . . . . . . . . . . . . . . . . . . . . . . 82

6.3.1 Results for the subsonic flow at $\mathrm{M}=0.3 \ldots \ldots \ldots$. . . . . . 82

6.3.2 Results for the transonic flow at $\mathrm{M}=0.8 \ldots \ldots \ldots$. . . . . . 84 
6.3.3 Remarks on the ROM efficiency . . . . . . . . . . . . . . . . 84

6.4 Conclusions . . . . . . . . . . . . . . . . . . . . . 88

$\begin{array}{llr}7 & \text { Conclusions and future work } & 89\end{array}$

$\begin{array}{lr}\text { Appendices } & 97\end{array}$

$\begin{array}{lr}\text { A Inviscid fluid solver Jacobians } & 98\end{array}$

$\begin{array}{ll}\text { B Theodorsen unsteady aerodynamics } & 101\end{array}$

$\begin{array}{ll}\text { C Inviscid aerodynamic solver validation } & 104\end{array}$

$\begin{array}{ll}\text { D Viscous aerodynamic solver validation } & 106\end{array}$

D.1 2D Zero pressure gradient flat plate (2DZP) . . . . . . . . . . . 106

D.2 2D Airfoil near wake (2DANW) . . . . . . . . . . . . . 108

D.3 2D NACA 4412 airfoil trailing edge separation (2DN44) $\ldots \ldots \ldots \ldots$

D.4 2D NACA 0012 airfoil (2DNO0) . . . . . . . . . . . . . . . . . . 114

D.5 2D NACA 0012 Transonic comparison with experiment . . . . . . . 117

D.6 2D NACA0012 forced oscillation comparison with inviscid simulations . 118 


\section{List of Figures}

1.1 The Collar triangle . . . . . . . . . . . . . . . . . . . . . 1

1.2 Sketch showing the ROM and full numerical solver time intervals . . . . 4

2.1 Adaptive ROM Flowchart . . . . . . . . . . . . . . . . . . . . . 11

3.1 Finite volume discretization . . . . . . . . . . . . . . . . . 17

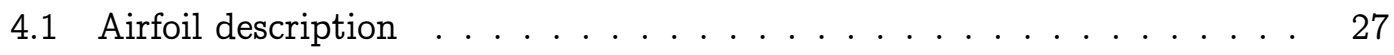

4.2 Non-inertial reference frame . . . . . . . . . . . . . . . . . 30

4.3 Mesh deformation example using Luke and Blades deformation method 31

5.1 Horizontal tail section sketch . . . . . . . . . . . . . . . 33

5.2 Airfoil mesh geometry . . . . . . . . . . . . . . . . . 34

5.3 Airfoil mesh . . . . . . . . . . . . . . . . . . . . . . . 35

5.4 Sub-mesh selection . . . . . . . . . . . . . . . . . 37

5.5 Rescaled forcing function for the pitch and heave motions, plotting the rescaled angle of attack and height vs. $f \cdot t \ldots \ldots 38$

5.6 POD on the fly vs. the CFD solver, with $\mathrm{M}=0.2$ at sea level, considering pitch motion with $a=0.01 \mathrm{rad}$ and $f=2.5 \mathrm{~Hz} \ldots \ldots . \ldots 39$

5.7 Counterpart of figure 5.6-top for $a=0.1 \mathrm{rad}$ and $f=10 \mathrm{~Hz}(k=0.48) . \quad 40$

5.8 Complete color maps and blow up near the leading edge of the instantaneous (rescaled) pressure distributions of the run in figure 5.7 at the minimum angle of attack $(f t=1 / 2)$, as obtained using the CFD alone and the POD on the fly method . . . . . . . . . . . . . 41

5.9 Counterpart of figure 5.6-top for $\mathrm{M}=0.8$, considering the following values of the forcing amplitude and frequency: $a=0.01 \mathrm{rad}, f=2.5 \mathrm{~Hz}$, $k=0.029 ; a=0.1 \mathrm{rad}, f=2.5 \mathrm{~Hz}, k=0.029$ and $a=0.1 \mathrm{rad}, f=40$ $\mathrm{Hz}, k=0.46 \ldots \ldots \ldots \ldots \ldots \ldots$

5.10 Counterpart of figure 5.8 for the run considered in figure 5.9-middle plot at times with a minimum and zero angle of attack . . . . . . . .

5.11 POD modes number evolution along the various timesteps performed by the POD on the fly ROM for the $\mathrm{M}=0.8$ case. . . . . . . . . . . . .

$5.12 \Delta T^{\mathrm{CFD}}$ effect on the ROM efficiency and number of retained modes for the $\mathrm{M}=0.2$ pitch motion with $a=0.01 \mathrm{rad}$ and $f=2.5 \mathrm{~Hz} \ldots \ldots$. 
$5.13 \varepsilon_{1}$ effect on the ROM efficiency and number of retained modes for the $\mathrm{M}=0.2$ pitch motion with $a=0.01 \mathrm{rad}$ and $f=2.5 \mathrm{~Hz} \Delta T^{\mathrm{CFD}}=0.10$.

$5.14 \varepsilon_{1}$ effect on the ROM efficiency and number of retained modes for the $\mathrm{M}=0.2$ pitch motion with $a=0.01 \mathrm{rad}$ and $f=2.5 \mathrm{~Hz} \Delta T^{\mathrm{CFD}}=0.005$.

$5.15 \mathcal{Q}_{0}$ effect on the ROM efficiency and number of retained modes for the $\mathrm{M}=0.2$ pitch motion with $a=0.01 \mathrm{rad}$ and $f=2.5 \mathrm{~Hz} . \Delta T^{\mathrm{CFD}}=0.10$,

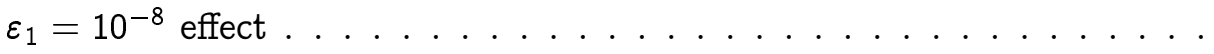

$5.16 \mathcal{Q}_{0}$ effect on the ROM efficiency and number of retained modes for the $\mathrm{M}=0.2$ pitch motion with $a=0.01 \mathrm{rad}$ and $f=2.5 \mathrm{~Hz} . \Delta T^{\mathrm{CFD}}=0.10$, $\varepsilon_{1}=10^{-12}$ effect

$5.17 \mathcal{Q}_{0}$ effect on the ROM efficiency and number of retained modes for the $\mathrm{M}=0.2$ pitch motion with $a=0.01 \mathrm{rad}$ and $f=2.5 \mathrm{~Hz} . \Delta T^{\mathrm{CFD}}=0.005$,

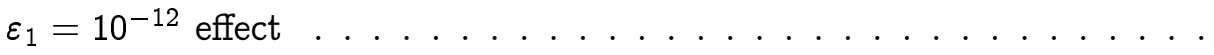

5.18 Comparison between Gauss Newton residual minimization and Galerkin projection for the $L_{E}$ and $\ell^{2}$ inner product selection effect on the ROM efficiency and number of retained modes for the $M=0.2$ pitch motion with $a=0.01 \mathrm{rad}$ and $f=2.5 \mathrm{~Hz} . \Delta T^{\mathrm{CFD}}=0.10, \varepsilon_{1}=10^{-12}$ effect $\ldots \ldots$

5.19 Comparison between Gauss Newton residual minimization and Galerkin projection for the $L_{E}$ and $\ell^{2}$ inner product selection effect on the ROM efficiency and number of retained modes for the $M=0.2$ pitch motion with $a=0.01 \mathrm{rad}$ and $f=2.5 \mathrm{~Hz} . \Delta T^{\mathrm{CFD}}=0.005, \varepsilon_{1}=10^{-12}$ effect $\ldots \ldots$

$5.20 E_{\text {res }}$ vs. $E_{\text {projres }}$ for a Galerkin based ROM residual minimization based

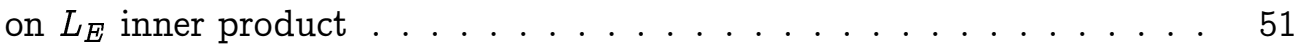

5.21 Airfoil normal modes . . . . . . . . . . . . . . . . 52

5.22 Frequency and damping evolution with incoming flow sped of the aeroelastic system (vg-vf flutter plot) at $\mathrm{M}=0.2$ and $\mathrm{M}=0.3 \ldots \ldots 5$

5.23 POD on the fly vs. the CFD solver. $\mathrm{M}=0.2$ at a speed below flutter point 54

$5.24 \mathrm{POD}$ on the fly vs. the CFD solver. $\mathrm{M}=0.2$ at a speed above flutter point 55

$5.25 \mathrm{~K}$ effect on control surface rotation, for $\mathrm{M}=0.2$ at a speed below flutter

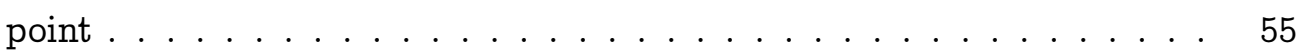

5.26 POD on the fly vs. the CFD solver. $\mathrm{M}=0.8$ at a speed below flutter point 56

$5.27 \mathrm{POD}$ on the fly vs. the CFD solver. $\mathrm{M}=0.8$ at a speed above flutter point 57

5.28 Iso Mach contours during simulation at $\mathrm{M}=0.8 \ldots \ldots . \ldots 57$

5.29 Number of retained modes and ROM vs NS times ratio for the $\mathrm{M}=0.2$ aeroelastic cases . . . . . . . . . . . . . . . . 58

5.30 Number of retained modes and ROM vs NS times ratio for the $\mathrm{M}=0.8$ aeroelastic cases . . . . . . . . . . . . . . . . . . 59

$5.31 K$ Effect on the number of retained modes and ROM vs NS times ratio for the $\mathrm{M}=0.2$ stable case . . . . . . . . . . . . . . . 59

6.1 Variable succesive gradients and Limiter effect on the scheme's face values 64

6.2 Sweby's diagram and Van Leer limiter . . . . . . . . . . . . . . 65 
6.3 Typical triangular unstructured mesh with the presence of a virtual cell to estimate the ratio of successive gradients . . . . . . . . . . 65

6.4 Airfoil mesh geometry . . . . . . . . . . . . . . . . . . 68

6.5 NACA0012 Airfoil mesh used for turbulent simulations . . . . . . . . . 70

6.6 Sub-mesh selection . . . . . . . . . . . . . . . . . 70

6.7 Rescaled forcing function for the pitch and heave motions, plotting the rescaled angle of attack and height vs. $f \cdot t \ldots \ldots \ldots \ldots 72$

6.8 POD on the fly vs. the $\mathrm{CFD}$ solver, with $\mathrm{M}=0.3$ at sea level, considering pitch motion with $a=0.01 \mathrm{rad}$ and $f=10.0 \mathrm{~Hz}(k=0.32) \ldots \ldots$.

6.9 Number of retained modes and ROM vs NS times ratio for the $\mathrm{M}=0.3$ at sea level, $a=0.01 \mathrm{rad}$ and $f=10.0 \mathrm{~Hz} \ldots \ldots \ldots . \ldots 74$

6.10 Counterpart of figure 6.8 for $a=0.1 \mathrm{rad} \ldots \ldots \ldots . \ldots 75$

6.11 Number of retained modes and ROM vs NS times ratio for the $\mathrm{M}=0.3$ at sea level, $a=0.1 \mathrm{rad}$ and $f=10.0 \mathrm{~Hz} \ldots \ldots \ldots . \ldots 75$

6.12 POD on the fly vs. the CFD solver, with $\mathrm{M}=0.8$ at sea level, considering pitch motion with $a=0.01 \mathrm{rad}$ and $f=2.5 \mathrm{~Hz} \ldots \ldots . \ldots 77$

6.13 Number of retained modes and ROM vs NS times ratio for the $\mathrm{M}=0.8$ at sea level, $a=0.01 \mathrm{rad}$ and $f=2.5 \mathrm{~Hz} \ldots \ldots \ldots \ldots 77$

6.14 POD on the fly vs. the CFD solver, with $\mathrm{M}=0.8$ at sea level, considering pitch motion with $a=0.01 \mathrm{rad}$ and $f=10.0 \mathrm{~Hz} \ldots \ldots \ldots$

6.15 Number of retained modes and ROM vs NS times ratio for the $M=0.8$ at sea level, $a=0.01 \mathrm{rad}$ and $f=10.0 \mathrm{~Hz} \ldots \ldots \ldots \ldots$

6.16 Turbulence solution strategy effect on the number of retained modes and ROM vs NS times ratio for the $\mathrm{M}=0.3, \mathrm{a}=0.1 \mathrm{rad}$ and $\mathrm{f}=10 \mathrm{~Hz}$ case . .

6.17 Turbulence solution strategy effect on the number of retained modes and $R O M$ vs NS times ratio for the $\mathrm{M}=0.8, a=0.01 \mathrm{rad}$ and $\mathrm{f}=10 \mathrm{~Hz}$ case . .

6.18 $\Delta T^{\mathrm{CFD}}$ effect on the number of retained modes and ROM vs NS times ratio for the $\mathrm{M}=0.3, \mathrm{a}=0.1 \mathrm{rad}$ and $\mathrm{f}=10 \mathrm{~Hz}$ case $\ldots \ldots$. . . . . 80

$6.19 \varepsilon_{1}$ effect on the number of retained modes and ROM vs NS times ratio for the $\mathrm{M}=0.3, \mathrm{a}=0.1 \mathrm{rad}$ and $\mathrm{f}=10 \mathrm{~Hz}$ case $\ldots \ldots \ldots$

6.20 Convection differencing scheme effect on the number of retained modes and $\mathrm{ROM}$ vs NS times ratio for the $\mathrm{M}=0.3, \mathrm{a}=0.1 \mathrm{rad}$ and $\mathrm{f}=10 \mathrm{~Hz}$ case

6.21 POD on the fly vs. the CFD solver. $\mathrm{M}=0.3$ at a speed below flutter point 82

6.22 Number of retained modes and ROM vs NS times ratio for the $\mathrm{M}=0.3$ at a speed below flutter point

6.23 POD on the fly vs. the CFD solver. $M=0.3$ at a speed above flutter point 83

6.24 Number of retained modes and ROM vs NS times ratio for the $\mathrm{M}=0.3$ at a speed above flutter point . . . . . . . . . . . . . . 84

6.25 POD on the fly vs. the CFD solver. $\mathrm{M}=0.8$ at a speed below flutter point 85

6.26 Number of retained modes and ROM vs NS times ratio for the $\mathrm{M}=0.8$ at a speed below flutter point . . . . . . . . . . . . . 85 
6.27 POD on the fly vs. the CFD solver. $M=0.8$ at a speed above flutter point 86

6.28 Number of retained modes and ROM vs NS times ratio for the $\mathrm{M}=0.8$ at a speed above flutter point . . . . . . . . . . . . . 86

6.29 $\Delta T^{\mathrm{CFD}}$ effect on the number of retained modes and ROM vs NS times ratio for the $\mathrm{M}=0.3$ case at a speed below the flutter point $\ldots \ldots$. . 87

$6.30 \varepsilon_{1}$ effect on the number of retained modes and ROM vs NS times ratio for the $\mathrm{M}=0.3$ case at a speed below the flutter point . . . . . . . . 87

C.1 Lift comparison between the CFD solver and Theodorsen analytical expressions . . . . . . . . . . . . . . . . . . 105

C.2 Lift comparison between the CFD solver and Theodorsen analytical expressions . . . . . . . . . . . . . . . . . . 105

D.1 2D Zero pressure gradient flat plate geometry description . . . . . . . 107

D.2 2DZP Mesh Description . . . . . . . . . . . . . . . . . . 107

D.3 2DZP Comparison between theoretical and CFD Obtained boundary layer velocity profile at $\mathrm{x}=0.97$, with different convection differencing schemes . . . . . . . . . . . . . . . . . . . . . . 108

D.4 2DANW Boundary conditions . . . . . . . . . . . . . . . 109

D.5 2DANW Mesh detail . . . . . . . . . . . . . . . . . . . . . . 109

D.6 Velocity profiles at different $x$ locations in the airfoil wake at $y=0$. . . 110

D.7 Velocity profiles at different $x$ locations in the airfoil wake . . . . . . . 110

D.8 2DN44 Boundary conditions . . . . . . . . . . . . . . . . . . . 111

D.9 2DN44 Boundary conditions . . . . . . . . . . . . . . . . . . . 111

D.10 2DN44 Velocity profiles along different lines in the vicinity of the trailing edge . . . . . . . . . . . . . . . . . . . 112

D.11 2DANW CFD Obtained pressure and velocity maps . . . . . . . . . 113

D.12 2DN44 Experimental and CFD generated velocity map and streamlines 113

D.13 2DN00 Boundary conditions . . . . . . . . . . . . . . . . . . . . . 114

D.14 2DN00 Mesh detail . . . . . . . . . . . . . . . . . . . . . . . . . . 114

D.15 2DN00 Obtained $C_{L}$ superimposed on top of available experimental data 115

D.16 Experimental (upper surface) and CFD generated pressure distributions over the airfoil for $\alpha=0^{\circ}, \alpha=10^{\circ}$ and $\alpha=15^{\circ} \ldots \ldots \ldots$

D.17 NACA0012 at $\mathrm{M}=0.756$, pressure data comparison between $\mathrm{CFD}$ and experimental data for $\alpha=0^{\circ}$ and $\alpha=1.95^{\circ} \ldots \ldots \ldots \ldots$

D.18 2D NACA0012. Lift comparison between inviscid and viscous turbulent simulations at $\mathrm{M}=0.30$ and $\mathrm{M}=0.80 \ldots \ldots \ldots \ldots \ldots$ 


\section{Chapter 1}

\section{Introduction and targets}

Aeroelasticity is defined as the science that studies the mutual interaction between the aerodynamic, inertial, and structural forces (Bisplinghoff et al., 1955). The importance of each contribution catalogs the possible phenomena and was schematically described in 1946 by Collar (Collar, 1978) in the famous Collar triangle (see figure 1.1), which is associated with dynamic aeroelastics; static aeroelasticity, instead, only involves steady aerodynamic and structural forces. Aeroelastic phenomena are of interest in a variety of fields, ranging from energy harvesting, both in the macro-scale (Xiao and Zhu, 2014) and in the micro-scale (Sousa et al., 2011), to biophysics, to e.g., understand snoring (Ellis, 2011). Although the most famous example of aerolastic instability is the Tacoma Narrows Bridge collapse in 1940 (Billah and Scanlan, 1991), the interest in dynamic aeroelasticity in aeronautics can be traced back to 1916, when the first recorded and documented flutter case occurred in a Handley Page O/400 twin engine

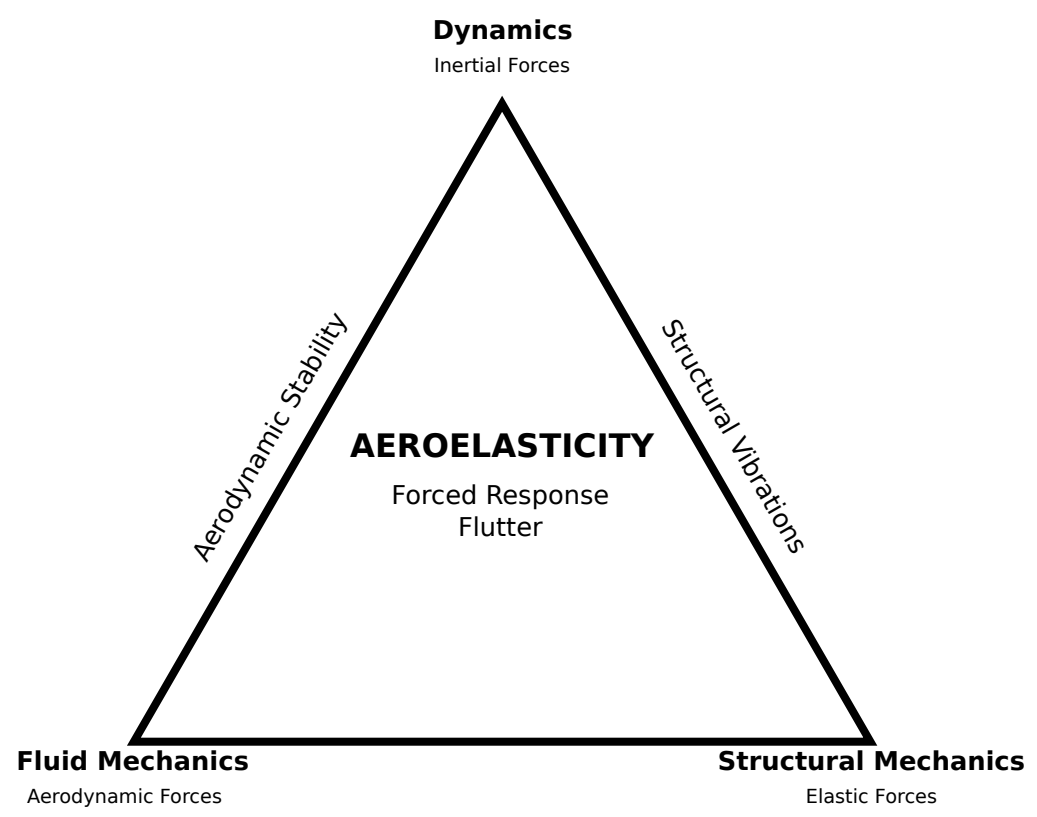

Figure 1.1: The Collar triangle 
bomber $^{1}$. Flutter findings in flight were especially important during the air races and speed records era (Tolve, 1975), as flight speed kept increasing and there was little or no knowledge on the phenomenon. Nowadays, aeroelastic stability certification requirements for transport category airplanes are clearly mandated by the airworthiness regulatory organisms like FAA (14 CFR 25.629) and EASA (CS 25.629).

Aeroelastic interaction frequently promotes instability. The nature and the consequences of the instability strongly depend on the particular problem, namely both on the incoming flow, especially on its Mach number and on whether it is steady or not, and on the topology (number of connected obstacles) and shape of the solid (whether it is slender or bluff). The aeroelastic equations can be seen as a dynamical system ((Holmes, 2005) and references therein). From this perspective, local instabilities of a given state, either steady or periodic, can be analyzed by linearizing the governing equations around the state. The instability sets in as the linearized system exhibits zero damping rate, which is analyzed through the eigenvalues of the Jacobian or the monodromy matrix for steady and periodic states, respectively. Near the instability threshold, as a given parameter such as the incoming velocity is increased, new states of the system come into play that are associated with the nearly unstable modes, a process known as bifurcation. The bifurcated solutions may either be stable or unstable, two situations that are known as super-critical or subcritical bifurcations, respectively. As the instability threshold is passed, supercritical bifurcations promote smooth transitions from the state of the system that is loosing instability. Subcritical bifurcations, instead, promote large drifts from the nearly unstable state that may be catastrophic in some cases.

Aeroelastic instabilities are most frequently oscillatory. Supercritical instabilities yield small oscillations near threshold that are relevant both in connection with fatigue, and strength substantiation if the speed increases abruptly. Subcritical instabilities lead to large departures from the basic state (even near threshold) that may lead to a collapse of the structure. Roughly speaking ${ }^{2}$, aeroelastic instabilities are classified into two categories:

- Flutter occurs for steady incoming flow, as an oscillatory instability (Hopf bifurcation) of the associated steady state that promotes self-sustained oscillations sets in. These oscillations result from the interplay of aerodynamic and elastic forces, including damping. Flutter, in turn, can be classified (Dowell, Crawley, et al., 1995; Razak et al., 2011) as binary flutter (the simplest case), stall flutter (which involves oscillations of either separation points or shock wave locations), and galloping flutter (transverse oscillations when aerodynamic forces are in phase with

\footnotetext{
${ }^{1}$ For this and additional excellent descriptions of events see Rodden (Theoretical and computational aeroelasticity, 2011)

${ }^{2}$ The literature is somewhat confusing in connection with this classification and history does not help. For instance, the word 'galloping' was first used for the famous Tacoma bridge, which was called 'galloping gertie' by construction workers due to its unusual oscillatory behavior; however, the collapse of the bridge is now attributed to binary flutter
} 
the elastic motion).

- Buffeting (Dowell, Crawley, et al., 1995) refers to instabilities promoted by unsteady (gusty or turbulent) incoming flows. In the aerospace industry, the most common form of buffet is the separation of the flow on the wing, and the impingement of the turbulent separated flow on the horizontal tail, leading typically to high loads and potentially to loss of control.

As appealing and useful as it is, especially for aeroelastic models exhibiting a few degrees of freedom, linearized theory is problematic for realistic descriptions. On the one hand, deriving the linearized equations of these descriptions (including the Navier-Stokes plus dynamic elasticity equations) is not an easy task. On the other, linearized analysis only provides the instability threshold, not the state of the system beyond threshold and, in particular, not the subcritical/supercritical character of the instability. Fully nonlinear detailed analysis of these phenomena relies on quite computationally expensive numerical simulations.

Among the inertial, structural, and aerodynamic forces that contribute to aeroelastic instability, it is the aerodynamic forces that aeroelasticians have more difficulties to model and simulate. A current industry standard is the use of the doublet lattice model (DLM) (Bisplinghoff et al., 1955), which is a linear unsteady, potential theory based, finite element method for modeling oscillating interfering lifting surfaces in subsonic flows (Rodden et al., 1998). For transonic flows, the usual approach is to generate corrections suggested by steady computational fluid dynamics (CFD) (Moreno et al., 2015), but the limited accuracy of such final model is uncertain and usually leads to extremely conservative analyses/results. This deficiency, and the fact that nonlinear aerodynamics are neglected, is promoting a demand from the industry for better aerodynamic models using plain CFD.

Prediction of steady forces over lifting surfaces and aerodynamic bodies by means of CFD is becoming a standard in aeronautical industry. It allows for accurately predicting the aircraft lift, drag, loads, and handling qualities, among others. Moreover, this is done at an affordable computational cost with the support of a decreasing number of testing campaigns, which are mainly needed to tune some solver parameters, associated with, e.g., turbulence models.

The use of CFD to predict unsteady aerodynamic forces for aeroelastic simulations requires, however, a huge computational effort and its accuracy is not guaranteed by the large majority of current solvers. This is due to the complexity of the flow, as highlighted in recent meetings on the subject (Heeg et al., 2013). The research community is making a tremendous effort on improving the unsteady CFD solvers available to the aeroelastic engineers. In some cases, a good correlation with wind tunnel tests has been reported (Moreno et al., 2012), but the computational cost still prevents them from being implemented in the industry. Increasing attention is being paid to the use of reduced order models (ROMs) in aeroelastic calculations. However, current ROMs for aeroelasticity, still rely somehow on the linearization of the aerodynamic forces. 
The resulting linearized equations are treated using various reduced order modeling methods, such as proper orthogonal decomposition (POD) (Dowell et al., 2001), Volterra kernels (Silva and Bartels, 2004), and frequency response functions (Raveh, 2004). Thus, these linearized models still exhibit some of the same drawbacks mentioned above in connection with DLM.

Fully nonlinear reduced order models have been already developed in various fields, including aerodynamics. These models take advantage of the well-known fact that in extended systems (modeled by partial differential equations and systems), the number of physically relevant degrees of freedom is much smaller than the number of numerical degrees of freedom (i.e. grid points or cells) required by standard CFD solvers. This is because of the redundancies associated with the underlying physical laws (e.g., mass and momentum conservation in the Navier-Stokes equations). A large class of ROMs first identify the physically relevant degrees of freedom by using POD and extracting the most energetic POD modes and then project the governing equations (or the CFD numerical scheme) onto these modes. Most ROMs based on this idea are designed to simulate attractors (not transients) and could be called pre-processed ROMs, because POD modes are calculated from a CFD run in a pre-process step. In principle, the CFD run should cover the relevant dynamics to be approximated by the ROM; this strategy can be improved using additional ingredients for fluid flows (Balajewicz et al., 2013).

A different perspective has been tested for simpler equations such as the Complex Ginzburg-Landau equation (Rapún et al., 2015), a paradigm of pattern forming systems that exhibits quite complex dynamic behaviors. The method consists in an adaptive strategy in which POD modes are calculated on demand as the simulation proceeds; this strategy has been proven very robust in both calculating particular solutions and constructing bifurcation diagrams (Terragni and Vega, 2014) that require a large number of particular time-dependent runs. The method uses both a numerical solver and a reduced order model in interspersed time intervals (see figure 1.2). The numerical solver is used to compute appropriate snapshots, which in turn are used to first calculate (at the outset of the temporal run) and later update (when needed) the POD manifold.

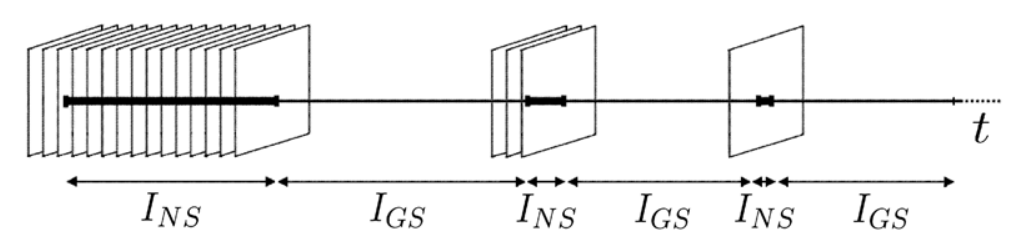

Figure 1.2: Sketch showing the ROM and full numerical solver time intervals

The ROM, which as mentioned is obtained upon projection of the governing equations onto the POD modes, is much less computationally expensive than the numerical solver and is intended to be used for the majority of the time. In other words, the adaptive strategy is implemented in a numerical scheme capable of switching seam- 
lessly between the full integration of the governing equations and an adaptive ROM that guarantees to capture the present simulation dynamics.

Note that such adaptive strategy requires two main ingredients:

- The necessity of updating the POD manifold must be detected using only information from the ROM. Appropriate a priori error/residual estimates can be used to this end.

- A convenient updating strategy of the POD modes at each updating event is required.

Additionally, the computational cost required to project the governing equations (especially, nonlinear terms) can be further reduced if only a given set of cells, where the main dynamics of the problem resides, are utilized. Such reduction of the computational cost has been used in the reduced modeling of several systems arising in non-linear elasticity (Ryckelynck, 2009) and steady aerodynamic forces (Alonso et al., 2009, 2010), as well as unsteady incompressible flows (Terragni et al., 2011). In particular, for the Complex Ginzburg-Landau equation, these ideas enable CPU acceleration factors in the range of 10-15 and 300-350 for simulations in one and two space dimensions, respectively.

Against this background, this thesis presents the extension of the above mentioned ideas to the simulation of unsteady, compressible, aeroelastic flows. This extension will be performed in various steps, solving the difficulties one after another. On the one hand, the method will be extended physically without, and then with, elastic deformation of the solid. On the other, the method will be first applied to inviscid and viscous $2 \mathrm{D}$ flows.

This thesis is organized as follows. Chapter 2 introduces the theoretical background behind POD based ROMs, including the new approaches required to make the method adaptive and useful in unsteady aerodynamic simulations. Chapter 3 describes the equations governing the unsteady behavior of the fluid. Chapter 4 describes the equations governing the dynamic behavior of a body immersed in a fluid, as well as the coupling between the fluid and structural solver through the interface forces and the numerical mesh deformation. It also shows the application to a particular case in which the body represents an airfoil capable of pitching and heaving coupled to a control surface that can rotate around a given hinge line. Chapters 5 and 6 exercise the newly developed method for inviscid and turbulent flows, respectively, at both subsonic and transonic regimes. First, the forces acting on an airfoil with a prescribed enforced motion are evaluated. Then, the complete coupled fluid structure interaction problem is studied by analyzing the impulse response of the airfoil. The thesis is finalized with Chapter 7, where some concluding remarks and future research topics are detailed. 


\section{Chapter 2}

\section{Theoretical background}

\subsection{Fluid structure interaction}

In a coupled fluid-structure interaction problem, the position and speed of each point and cell in the computational domain $\Omega$ depend on the body motion. At the same time, the body motion depends on the internal elastic, viscous, inertial and control forces, as well as on the forces acting on the boundary shared with the fluid.

The equations governing the problem can be written as

$$
\begin{array}{r}
\frac{\partial\left(\mathcal{V}_{i}(\tilde{x}, t) \mathcal{Q}_{i}\right)}{\partial t}+\mathcal{R}_{i}(\mathcal{Q}, \tilde{x})=0 \\
\mathcal{M} \frac{\partial^{2} \tilde{x}}{\partial t^{2}}+\mathcal{B} \frac{\partial \tilde{x}}{\partial t}+\mathcal{K} \tilde{x}+\phi(\tilde{x})=\mathcal{F}(\mathcal{Q})
\end{array}
$$

where $\Omega$ is discretized in $i=1, . ., M$ cells of volume $\mathcal{V}_{i}$ and $\mathcal{Q}_{i}=\{\rho, u, v, w, p\}_{i}$ is the state vector joining the density, speeds and pressure of the fluid, respectively. The equations are originally written in the conserved variables $(\rho, \rho u, \rho v, \rho v, \rho E)$ and later transformed to primitive variables, as shown in §3.8. A fluid state equation is additionally required to close the problem. Finally, a suitable algorithm to move and deform the mesh, so that it remains conformal to the body, is required.

The coordinates corresponding to the body motion are represented by $\tilde{x}$, and $\mathcal{M}, \mathcal{B}$ and $\mathcal{K}$ represent the mass, damping and stiffness matrices of the body, respectively. Finally, $\phi(\tilde{x})$ is a non-linear stiffness function, e.g., a free-play in a hinge, and $\mathcal{F}(\mathcal{Q})$ accounts for the generalized fluid forces on the body.

This problem is traditionally solved in a loosely coupled manner, since the characteristic time of the body motion is usually orders of magnitude higher than the aerodynamic counterpart one. In this way, the flow state is solved first, using the body state from the previous time step. The fluid forces acting on the body are computed and subsequently the body is evolved in time.

For the numerical simulations performed in this thesis, the fluid governing equations are evolved in time using an implicit, first order accurate, Euler algorithm, to which a 
pseudo-time sub-iteration step (Rumsey et al., 1996) has been added as

$$
\begin{aligned}
\left(\frac{\mathcal{V}_{i}^{n+1} \mathcal{Q}_{i}^{m+1, n+1}-\mathcal{V}_{i}^{n+1} \mathcal{Q}_{i}^{m, n+1}}{\Delta \tau}\right. & \left.+\frac{\mathcal{V}_{i}^{n+1} \mathcal{Q}_{i}^{m+1, n+1}-\mathcal{V}_{i}^{n} \mathcal{Q}_{i}^{n}}{\Delta t}\right)+\mathcal{R}_{i}^{m} \\
& +\frac{\partial \mathcal{R}_{i}^{n}}{\partial \mathcal{Q}}\left(\mathcal{Q}^{m+1, n+1}-\mathcal{Q}^{m, n+1}\right)=0
\end{aligned}
$$

where $\Delta \tau$ denotes the pseudo time-step, which is varied for each cell based on a constant CFL number $(u \Delta \tau / \Delta x)$ (Courant et al., 1967), and $\mathcal{Q}_{i}^{m, n+1}$ identifies the $m$ - th approximation to $\mathcal{Q}_{i}^{n+1}$. The Jacobian might be approximated by a lower order discretization, or even a diagonal approximation.

The solution is iterated in $\mathrm{m}$ until enough convergence has been achieved, as

$$
\begin{aligned}
\left(\frac{\mathcal{V}_{i}^{n+1}}{\Delta \tau}+\frac{\mathcal{V}_{i}^{n+1}}{\Delta t}\right. & \left.+\frac{\partial \mathcal{R}_{i}^{n}}{\partial \mathcal{Q}_{i}}\right)\left(\mathcal{Q}_{i}^{m+1, n+1}-\mathcal{Q}_{i}^{m, n+1}\right)= \\
& -\left(\mathcal{R}_{i}^{m}+\frac{\mathcal{V}_{i}^{n+1} \mathcal{Q}_{i}^{m, n+1}-\mathcal{V}_{i}^{n} \mathcal{Q}_{i}^{n}}{\Delta t}\right) .
\end{aligned}
$$

After reaching convergence, for example based on the relative change of the state vector and the reduction of the residuals a given order of magnitude, the forces acting on the body, $\mathcal{F}(\mathcal{Q})$, are calculated and the body state is then evolved in time. The evolution of the body changes the definition of the computational domain mesh, which might imply the update of the cell volumes, $\mathcal{V}_{i}$, that is used to further carry on the fluid integration in the next time step.

\subsection{POD based reduced order model}

\subsubsection{POD decomposition}

Once the complete problem for the governing equations has been integrated for an adequate timespan, the flow state variables for each time step can be concatenated into a snapshot matrix, such as

$$
S=\left[\begin{array}{ccccc}
\mathcal{Q}_{1}^{1} & \mathcal{Q}_{1}^{2} & \cdots & \cdots & \mathcal{Q}_{1}^{T} \\
\mathcal{Q}_{2}^{1} & \mathcal{Q}_{2}^{2} & \cdots & \cdots & \mathcal{Q}_{2}^{T} \\
\vdots & \vdots & \vdots & \vdots & \vdots \\
\mathcal{Q}_{M}^{1} & \mathcal{Q}_{M}^{2} & \cdots & \cdots & \mathcal{Q}_{M}^{T}
\end{array}\right]
$$

The POD modes are calculated from this snapshot matrix. Traditionally, POD modes have been calculated by obtaining the covariance matrix of the snapshot matrix as $R_{i j}=\left\langle S_{i}, S_{j}\right\rangle$ for a given inner product $\langle\cdot, \cdot\rangle$. It is customary expressing the eigenvalue decomposition of $R$ as

$$
R A=A D^{2}
$$


where the columns of $A$ are the orthonormal eigenvectors of $R$ and $D^{2}$ is the diagonal matrix of the eigenvalues of $R$, whose square roots are the singular values. Then, the POD modes are the columns of the following matrix

$$
\Phi=S A D^{-1}
$$

so that they are orthonormal with the considered inner product.

An additional important property of the POD modes is that, if the snapshot matrix is approximated as $S^{N}$ making use of the first $N$ POD modes, the relative root mean square error (RRMSE), defined as

$$
R R M S E_{N}^{T}=\sqrt{\frac{1}{M T}\left(\sum_{i j} \frac{S_{i j}^{N}-S_{i j}}{S_{i j}}\right)^{2}}
$$

can be estimated as

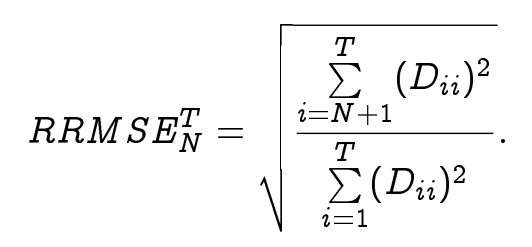

However, due to the fact that the covariance matrix requires the multiplication by the inner product of the snapshots, the POD modes associated with singular values such that $\left(D_{j j}\right) /\left(D_{11}\right)<10^{-8}$ are not valid (Rapún et al., 2015) with the standard double precision binary floating point format.

For the particular, and typical, case in which the inner product can be represented as

$$
\left\langle S_{i}, S_{j}\right\rangle=\left(S_{i}\right)^{T} \mathcal{G} S_{j}
$$

where $\mathcal{G}$ is a diagonal matrix, the accuracy of the POD modes is increased by avoiding the creation of the covariance matrix. In a first step, SVD decomposition is applied to

$$
\tilde{S}=\sqrt{\mathcal{G}} S
$$

so that

$$
\tilde{S}=U \Sigma V^{T}
$$

and therefore

$$
S=\sqrt{\mathcal{G}^{-1}} U \Sigma V^{T}
$$

Substituting these into the definition of the covariance matrix, and taking into account 
that $D$ and $\mathcal{G}$ are diagonal matrices, it follows that

$$
R=S^{T} \mathcal{G} S=V \Sigma U^{T} \sqrt{\mathcal{G}^{-1}} \mathcal{G} \sqrt{\mathcal{G}^{-1}} U \Sigma V^{T}=V \Sigma^{2} V^{T}
$$

Finally, comparing equation (2.6) and equation (2.14) it is trivial to show that

$$
\Phi=S V \Sigma^{-1}=\sqrt{\mathcal{G}^{-1}} U
$$

\subsubsection{Flow field as combination of POD modes}

For a given set of $N$ selected POD modes, the flow field can be expressed as a combination of the modes, where a reference value $\mathcal{Q}_{0}$ might potentially be used as

$$
\mathcal{Q}=\mathcal{Q}_{o}+\Phi \xi
$$

so that with the substitution into equation (2.3), it follows

$$
\sum_{j=1}^{N} \Phi_{i j}\left(\frac{\mathcal{V}_{i}^{n+1} \xi_{j}^{n+1}-\mathcal{V}_{i}^{n} \xi_{j}^{n}}{\Delta t}\right)+\sum_{k=1}^{M} \frac{\partial \mathcal{R}_{i}^{n}}{\partial \mathcal{Q}^{k}} \sum_{j=1}^{N} \Phi_{k j}\left(\xi_{j}^{n+1}-\xi_{j}^{n}\right)+\mathcal{R}_{i}^{n}=0
$$

which could be used to evolve the fluid equations.

\subsubsection{Residual minimization}

Unfortunately, the number of cells in which the dominion is divided is usually orders of magnitude higher than the number of modes retained, making computationally expensive the minimization of the residual. Further reduction needs to be applied to the system, which can be done in any of the following ways:

- Minimize the least squares residual on a subset of $M$ selected cells

$$
\min \left(\sum_{i=1}^{M}\left[\sum_{j=1}^{N} \Phi_{i j}\left(\frac{\mathcal{V}_{i}^{n+1} \xi_{j}^{n+1}-\mathcal{V}_{i}^{n} \xi_{j}^{n}}{\Delta t}\right)+\mathcal{R}_{i}\left(\mathcal{Q}_{0}+\Phi \xi^{n+1}\right)\right]^{2}\right)
$$

- Project the equation over the POD modes, using a defined inner product. This is known as Galerkin projection:

$$
\left\langle\Phi, \sum_{j=1}^{N} \Phi_{i j}\left(\frac{\mathcal{V}_{i}^{n+1} \xi_{j}^{n+1}-\mathcal{V}_{i}^{n} \xi_{j}^{n}}{\Delta t}\right)+\mathcal{R}_{i}\left(\mathcal{Q}_{o}+\Phi \xi^{n+1}\right)\right\rangle=0
$$

In this case, the definition of the inner product is essential, since it plays a key role in the characteristics of the equation above 


\subsection{Adaptive POD on the fly}

\subsubsection{POD based ROM drawbacks}

Despite the use of the POD projection based ROMs, the unsteady simulations would still be prohibitively expensive, in its original form, for their use on an industrial environment. In other words, the overhead of performing the POD decomposition over a several millions (a typical number of grid points) times several thousands (a possible number of snapshots) snapshots matrix has to be added to the cost of solving the fluid equations to calculate the snapshots.

Besides, the snapshots to create the ROM have been traditionally obtained from complete simulations time history. This allows to simulate problems with similar dynamics to the previous solutions but, since the full equations are not completely integrated, it is not able to capture behaviors of the system not previously encountered

On another note, POD based ROMs are known to traditionally suffer from stability problems, mainly due to the fact that the numerical scheme used to obtain the solution can be stable, but the projection on a truncated basis that does not cover the whole solution subspace traditionally is not. In this way, some modes that initially had very low energy become dominant in the solution and the ROM validity is jeopardized.

\subsubsection{POD on the fly}

The new methodology, introduced here in the context of unsteady aerodynamics and computational aeroelasticity, known as POD on the fly and previously presented in (Alonso et al., 2009, 2010; Rapún et al., 2015; Terragni and Vega, 2014; Terragni et al., 2011), overcomes these drawbacks.

A flowchart summarizing the particularities of the method can be seen in figure 2.1. Initially, the integration of the governing equations starts using the full numerical solver. Then, after a prescribed amount of time steps, for a time $T_{o}^{\mathrm{CFD}}$, have been completed, a POD basis is obtained and truncated to a number $\mathrm{N}$ of modes in such a way that

$$
R R M S E_{N}^{T} \leq \varepsilon_{1}
$$

where $R R M S E_{N}^{T}$ is defined in equation (2.9), and $\varepsilon_{1}$ is a tunable parameter.

At this point, the integration is switched to the ROM solver, and the system evolved in time, until the ROM loses its validity due to changes in system dynamics or truncation instabilities. It is therefore paramount to introduce in the time evolution the ability to detect this phenomenon. This is accomplished in this work by monitoring the residual reduction the ROM is able to achieve. In this way, the ROM integration will finish if

$$
E_{\text {res }}=\frac{\left\|\sum_{j=1}^{N} \Phi_{i j}\left(\frac{\mathcal{V}_{i}^{n+1} \xi_{j}^{n+1}-\mathcal{V}_{i}^{n} \xi_{j}^{n}}{\Delta t}\right)+\mathcal{R}_{i}\left(\mathcal{Q}_{o}+\Phi \xi^{n+1}\right)\right\|}{\left\|\mathcal{R}_{i}\left(\mathcal{Q}_{o}+\Phi \xi^{n}\right)\right\|}>\frac{1}{K}
$$




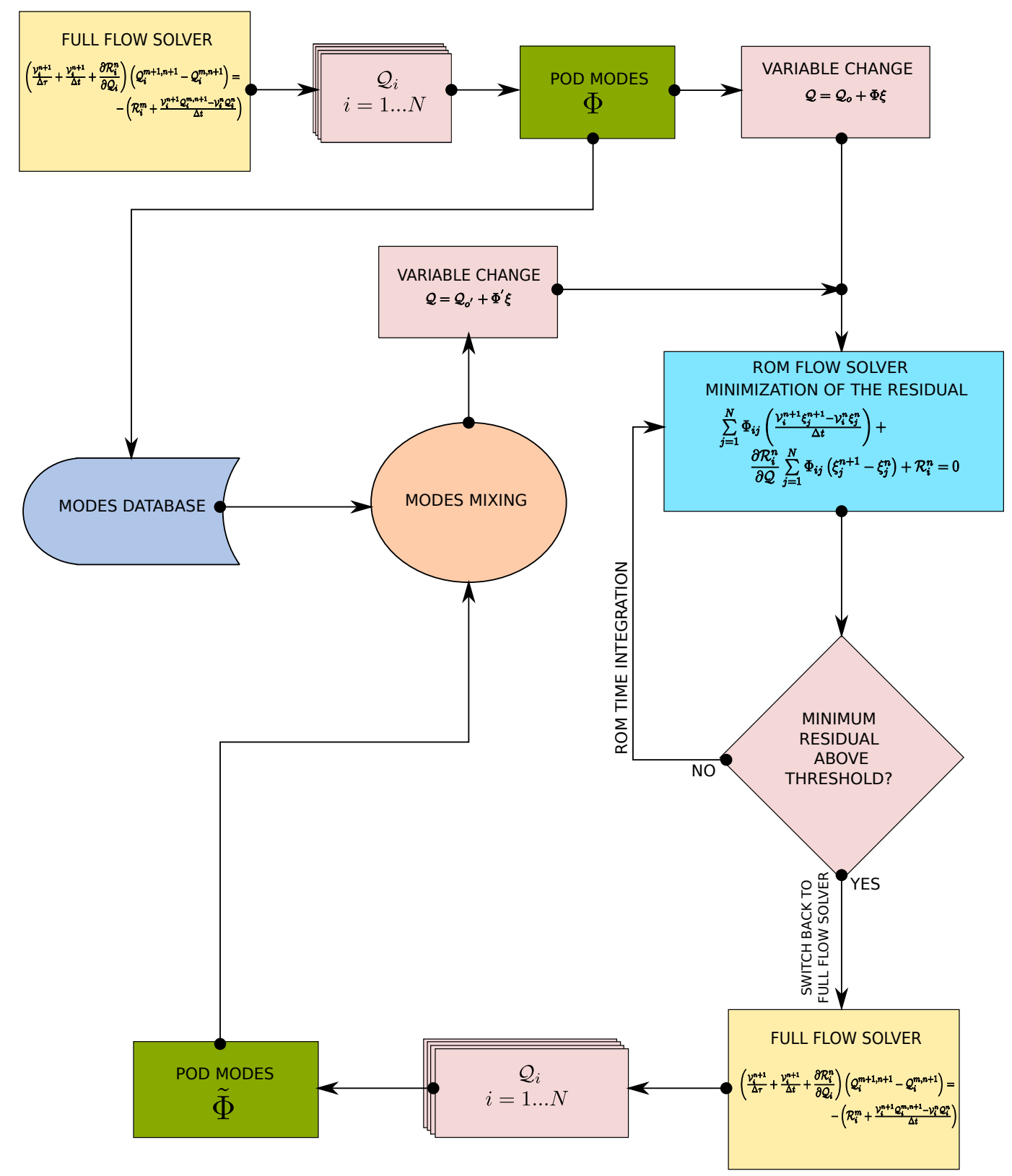

Figure 2.1: Adaptive ROM Flowchart 
where $K$ is a tunable parameter and $\|\cdot\|$ is associated with a previously defined inner product.

After switching back to the numerical solver, the system is evolved for a time $\triangle T^{\mathrm{CFD}}$. It must be noted that the selection of $\Delta T^{\mathrm{CFD}}$ needs to consider the balance between acquiring the new system dynamics and the efficiency of the overall integration scheme.

At the end of the period $\triangle T^{\mathrm{CFD}}$, the new system dynamics have been acquired, and a new POD basis $(\Phi)$ has been calculated from the corresponding matrix of snapshots. Since some of the previous system dynamics are most likely part of the present system evolution, both $\Phi$ and $\hat{\Phi}$ (the previous set of modes) need to be combined to create the new updated ROM. This is done by applying POD to the set of vectors

$$
\hat{\nu}_{1} \hat{\Phi}_{1}, \cdots, \hat{\nu}_{N_{\text {old }}} \hat{\Phi}_{N_{\text {old }}}, \nu_{1} \Phi_{1} \cdots, \nu_{N_{\text {new }}} \Phi_{N_{\text {new }}}
$$

where the weights $\hat{\nu}_{j}$ and $\nu_{j}$ are defined as

$$
\hat{\nu}_{j}=\min \left\{\frac{\hat{D}_{j j}}{\sqrt{\sum_{i=1}^{N_{\text {old }}}\left(D_{i i}\right)^{2}}}, \frac{\xi^{j}}{\sqrt{\sum_{i=1}^{N_{\text {old }}}\left(\xi^{j}\right)^{2}}}\right\}, \nu_{j}=\frac{D_{j j}}{\sqrt{\sum_{i=1}^{N_{\text {new }}}\left(D_{i i}\right)^{2}}} .
$$

In this way, those modes with higher relative "energy" (based on the inner product), and with higher relative contribution to the flow field, are retained in the newly created $\mathrm{ROM}$, and the process is ready to start over again.

\subsection{Definition of the inner product}

The inner product definition plays a key role in the definition of the ROM and its properties. In this work, the use of three different inner products has been explored. They are explained in the following subsections.

\subsubsection{Standard inner product $\ell^{2}$}

The standard inner product $\ell^{2}$ of two fluid state vectors is defined as

$$
\left\langle\mathcal{Q}_{i}, \mathcal{Q}_{j}\right\rangle=\sum_{c=1}^{M}\left(\rho_{i}^{c} \rho_{j}^{c}+u_{i}^{c} u_{j}^{c}+v_{i}^{c} v_{j}^{c}+w_{i}^{c} w_{j}^{c}+p_{i}^{c} p_{j}^{c}\right)=\left(\mathcal{Q}_{j}\right)^{T} \mathcal{I} \mathcal{Q}_{i}
$$

Examining the definition a bit closer, the lack of physical meaning is evident, since it adds variables with different units with no real relation to the system energy. In this way, POD modes obtained by using this inner product would ease in minimizing the total vector norm, but not necessarily conserve the system energy. 


\subsubsection{Standard inner product $L^{2}$}

The standard inner product $L^{2}$ of two fluid state vectors is defined as

$$
\left\langle\mathcal{Q}_{i}, \mathcal{Q}_{j}\right\rangle=\int_{\Omega}\left(\rho_{i} \rho_{j}+u_{i} u_{j}+v_{i} v_{j}+w_{i} w_{j}+p_{i} p_{j}\right) d \Omega
$$

For a cell-centered fluid solver discretization, like the one used in this work, it can be expressed as

$$
\left\langle\mathcal{Q}_{i}, \mathcal{Q}_{j}\right\rangle=\sum_{c=1}^{M} v^{c}\left(\rho_{i}^{c} \rho_{j}^{c}+u_{i}^{c} u_{j}^{c}+v_{i}^{c} v_{j}^{c}+w_{i}^{c} w_{j}^{c}+p_{i}^{c} p_{j}^{c}\right)=\left(\mathcal{Q}_{j}\right)^{T} \mathcal{V} \mathcal{Q}_{i},
$$

where $\mathcal{V}$ is the diagonal matrix formed by stacking the volumes for the different cells.

As in the $\ell^{2}$ case, the $L^{2}$ product has no physical meaning. It can be seen as a weighted $\ell^{2}$ product, with the advantage that the weights are equal to the residual definition in equation (2.17).

\subsubsection{Energy based $L_{E}$ inner product}

Several authors (Barone et al., 2009; Kalashnikova et al., 2014) highlight the importance of defining energy based inner products to increase the POD based ROM stability. Recalling that the total energy of the flow is expressed by

$$
\rho E=p \cdot \frac{\gamma}{\gamma-1}+\frac{1}{2} \cdot \rho\left(u^{2}+v^{2}+w^{2}\right)
$$

and that the POD based ROM in this work is referenced to the flow steady state, as expressed in equation (2.16), the inner product is defined as

$\left\langle\mathcal{Q}_{i}, \mathcal{Q}_{j}\right\rangle=\int_{\Omega}\left(\frac{\rho}{2}\left(u_{i} u_{j}+v_{i} v_{j}+w_{i} w_{j}\right)+\frac{\rho_{i} \rho_{j}}{2 \rho_{o}}\left(u_{o}^{2}+v_{o}^{2}+w_{o}^{2}\right)+\frac{p_{i} p_{j} \gamma}{p_{o}(\gamma-1)}\right) d \Omega$

This inner product resembles the flow total energy (it has energy units), but keeps a diagonal matrix based definition, with all the computational advantages stated before.

\subsubsection{Selection based inner product}

The main drawback of the traditional POD based ROMs is the computational overhead associated to the extraction of the POD modes. As previously highlighted, this cost is intrinsically associated to the definition of the inner product.

One way to overcome this drawback is (Alonso et al., 2009, 2010) to define the inner product only in a subset of the cells that form the computational domain in which the fluid is divided. By doing so, the evolution of the fluid variables for the non-selected cells is made slave to the ones of the selected domain. 
Recalling the definition of inner product in matrix form

$$
\left\langle S_{i}, S_{j}\right\rangle=\left(S_{i}\right)^{T} \mathcal{G} S_{j}
$$

and rearranging the vectors such that the $M$ selected subset cells are at the top, the $\mathcal{G}$ matrix is created such that

$$
\mathcal{G}=\operatorname{diag}\left(\mathcal{G}_{1}, \ldots, \mathcal{G}_{M}, 0, \ldots, 0\right)
$$

Since all the snapshots are used for the creation of the POD modes, a full reconstruction of the flow field is possible, while reducing significantly the cost associated to the extraction of the POD modes. Besides, the spatial derivatives corresponding to each of the POD modes, in the selected and slave cells, can be calculated and stored in order to further reduce the cost. Additionally, the residual equations for the rows corresponding to the non selected cells do not play any role in the minimization, so the cost can be further reduced by avoiding its calculation.

Due to the fact that the rows of the residual corresponding to the time evolution of the selected cells are calculated, but the residual depends on values from its neighbor cells, special care has to be put in choosing the cell selection, so that it allows for adequate imposition of the boundary conditions.

Also, due to the fact that the behavior of the non-selected cells is subordinated to the evolution of the selected cells, it is important to include in this selection those areas were significant and unique dynamics exist.

Lastly, it is typical of CFD solvers to accumulate errors in specific zones of the computational domain, like for example the trailing edges of lifting surfaces. By not selecting cells in this regions, the robustness and significance of the extracted modes is increased. 


\section{Chapter 3}

\section{Computational fluid dynamics solver}

\subsection{Introduction}

Due to the evident need, highlighted in the previous chapter, to have full control over the flow solver residual calculation, boundary condition enforcement, time integration and flow reconstruction, already available codes (OpenFoam and SU2, to cite some open source examples) were discarded and a new fluid dynamics solver developed. The finite volume method has been chosen due to its higher quality to computational cost ratio, and its simplicity in handling non structured meshes.

The new computational fluid dynamics (CFD) solver PyFlow (for lack of a better name) is written in Python, and consists of several classes, and methods associated to the classes, clustered along a main subroutine responsible of the variable definition, residual calculation, time integration, fluid-structure interaction and results output. Finally, an interface with ParaView (Ahrens et al., 2005) has been written to ease in the post-processing and visualization of the flow evolution.

\subsection{Governing equations}

The unsteady Reynolds averaged Navier-Stokes (URANS) equations may be written for a moving or stationary control volume in integral conservation form as:

- Conservation of Mass:

$$
\frac{d}{d t} \int_{\Omega} \rho d V+\int_{\Omega} \nabla \cdot(\rho \bar{u}) d V-\int_{\partial \Omega}(\rho \overline{\mathrm{w}}) \cdot d \bar{S}=0
$$

- Conservation of Momentum (neglecting body forces):

$$
\frac{d}{d t} \int_{\Omega} \rho \bar{u} d V+\int_{\Omega}(\nabla \cdot(\rho \bar{u} \otimes \bar{u})+\nabla p+\nabla \cdot \mathcal{T}) d V-\int_{\partial \Omega}(\rho \bar{u} \otimes \bar{w}) \cdot d \bar{S}=0
$$


- Conservation of total fluid energy:

$$
\begin{aligned}
\frac{d}{d t} \int_{\Omega} \rho E d V+\int_{\Omega}(\nabla \cdot(\bar{u}(\rho E))+\nabla \cdot(\bar{u} p)+ & \nabla \cdot(\mathcal{T} \cdot \bar{u})+\nabla \cdot j) d V \\
& -\int_{\partial \Omega}(\rho E \overline{\mathrm{w}}) \cdot d \bar{S}=0
\end{aligned}
$$

In the equations above, $\rho$ is the mean mass density, $\bar{u}=(u, v, w))$ is the mean fluid velocity, $p$ is the mean pressure, $E$ is the mean total energy density $\left(E=e+\frac{|\bar{u}|^{2}}{2}\right.$ with $e$ the specific internal energy), $\bar{w}$ represents the speed of the control volume face, $j=-k_{e} \nabla T$ is the diffusive flux of heat and $\mathcal{T}$ is the viscous stress tensor, represented making use of the Boussinesq eddy viscosity assumption (Boussinesq, 1877) as

$$
\begin{gathered}
D=\frac{1}{2}\left[(\nabla \bar{u})+(\nabla \bar{u})^{T}\right], \\
\mathcal{T}=-2 \mu_{e}\left(D-\frac{1}{3} \operatorname{tr}(D) I\right),
\end{gathered}
$$

where $\mu_{e}$ is the effective fluid viscosity and $k_{e}$ is the effective conductivity, i.e. they include the laminar and turbulent contributions.

The mean flow equations are closed by the equation of state for a calorically perfect gas, for which

$$
p=\rho R T, \quad e=c_{v} T=(\gamma-1) R T,
$$

where $R$ is the gas constant and $\gamma=c_{p} / c_{v}$ is the ratio of specific heats at constant pressure and volume, $c_{p}$ and $c_{v}$, respectively.

\subsection{Computational domain}

The computational domain discretization follows the work from Greenshields et al. (Greenshields et al., 2010), in which the spatial domain is divided into a number of contiguous control volumes (cells). There is no alignment, in general, with the coordinate system and the number of neighboring cells can vary from cell to cell. Each cell face is either internal, intersecting two cells, or external, if it is part of a boundary

As seen in figure 3.1, each face is assigned an owner and a neighbor cell. The face area vector $S_{f}$ is a vector normal to the face surface pointing away from the owner cell, whose magnitude is that of the area of the face. The vector $d$ connects the centroid of the owner cell $P$ to that of the neighboring cell $N$, and the vector $d_{f N}$ connects the center of the face to the centroid of the cell $\mathrm{N}$.

\subsection{Convective terms}

The convective terms at the cell faces in equations (3.1)-(3.3) are evaluated by interpolation of cell center values to the faces. The second-order semi-discrete, non-staggered schemes of Kurganov and Tadmor (KT)(Kurganov and Tadmor, 2000) and Kurganov, 
Noelle and Petrova (KNP)(Kurganov et al., 2001) ease the interpolation by only making use of the owner and neighbor cells values.

Upon closer inspection, the convective fluxes can be classified by whether they are divergence based or gradient based.

\subsubsection{Divergence based terms}

The treatment of the divergence terms $(\nabla \cdot \bar{u} q)$ in equations (3.1)-(3.3) is a critical aspect of the applied central scheme. Each term is integrated over the control volume by making use of the Gauss theorem, as follows

$$
\int_{\Omega} \nabla \cdot(\bar{u} q) d V=\int_{\partial \Omega}(\bar{u} q) \cdot d S \approx \sum_{f}\left(\bar{u}_{f} q_{f}\right) \cdot \bar{S}_{f}
$$

where $q$ is the convected variable.

If the above equation is combined with the terms that account for the volume control motion in equations (3.1)-(3.3), we finally have

$$
\int_{\Omega} \nabla \cdot(\bar{u} q) d V-\int_{\partial \Omega}(q \overline{\mathrm{w}}) \cdot d \bar{S} \approx \sum_{f}\left(\bar{u}_{f}-\overline{\mathrm{w}}\right) q_{f} \cdot \bar{S}_{f}=\sum_{f} q_{f} \phi_{f}
$$

where $\sum_{f}$ denotes a summation over the cell faces and $\phi_{f}$ is the volumetric flux, i.e. the volume of fluid flowing through the face per unit time. In essence, it can be seen that the convective fluxes must account for the relative reduction or enhancement of the flux through the control surface owing to the local control surface speed.

For incompressible flows, the values at the cell faces are usually calculated by linear interpolation of $\bar{u}$ from neighboring cells (central differencing) and interpolation of

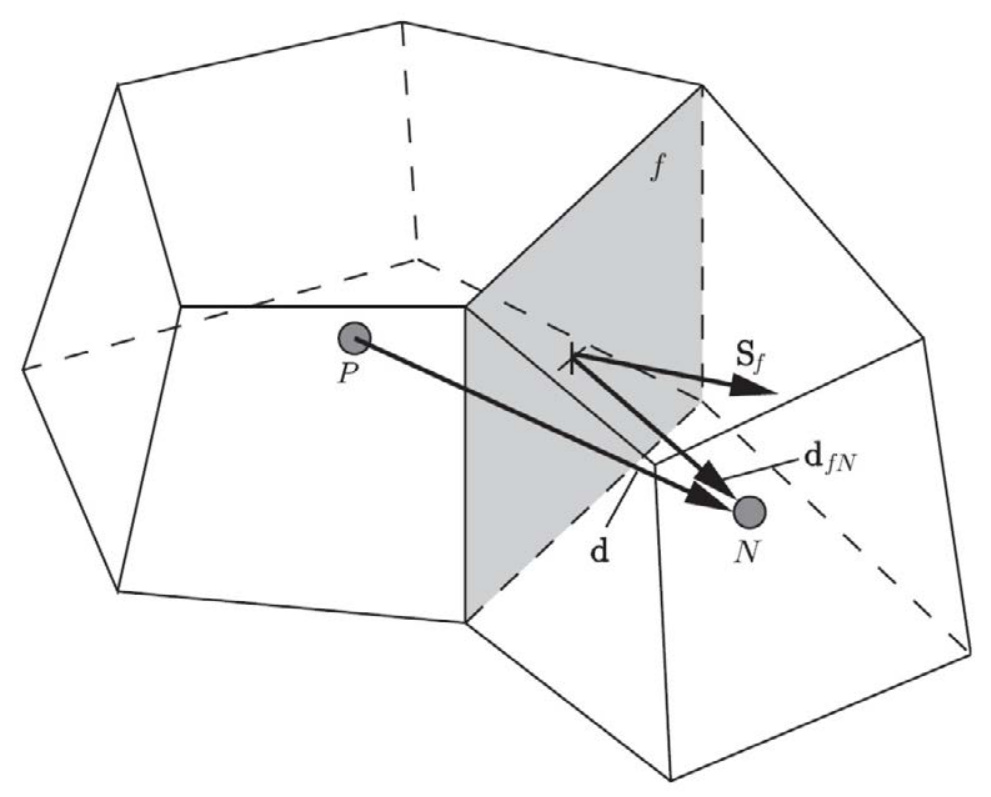

Figure 3.1: Finite volume discretization 
$q$ according to some upwinding scheme to stabilize the solution. For compressible flows, however, the fluid properties are not only transported by the flow, but also by wave propagation. This requires the flux interpolation to be stabilized based on the transport that can occur in any direction. The KNP method is implemented similarly to OpenFoam's rhoCentralFoam solver (Borm, 2010; Jasak et al., 2007), by splitting the interpolation procedure in two directions

$$
\sum_{f} q_{f} \phi_{f}=\sum_{f}\left[\alpha \phi_{P f} q_{P f}+(1-\alpha) \phi_{N f} q_{N f}+\omega_{f}\left(q_{N f}-q_{P f}\right)\right]
$$

where the subscripts $\mathrm{N}$ and $\mathrm{P}$ correspond to the owner and neighbor cells as illustrated in figure 3.1. The third term is strictly only required in cases where the convection term is a part of a substantive derivative, e.g. $\nabla \cdot(\rho \bar{u} \otimes \bar{u})$ in equation (3.2). It is an additional diffusion term using a volumetric flux $\omega_{f}$ based on the maximum speed of propagation of any discontinuity that might exist at a face between values interpolated in any direction.

The KNP method calculates $\alpha$ based on one-sided local speeds of propagation, and the weighting is additionally biased in the upwind direction. The volumetric fluxes associated with the local speeds of propagation are therefore defined as

$$
\begin{aligned}
& \psi_{P}=\max \left(\phi_{P f}+c_{f_{P f}}\left|S_{f}\right|, \phi_{N f}+c_{f_{N f}}\left|S_{f}\right|, 0\right), \\
& \psi_{N}=\min \left(\phi_{P f}-c_{f_{P f}}\left|S_{f}\right|, \phi_{N f}-c_{f_{N f}}\left|S_{f}\right|, 0\right),
\end{aligned}
$$

Here, $c_{f_{P N}}=\sqrt{\gamma R T_{P N}}$ is the speed of sound of the gas at the face. Finally, the weighting factor is calculated as

$$
\alpha=\frac{\psi_{P}}{\psi_{P}+\psi_{N}}
$$

and the diffusive volumetric flux is given by

$$
\omega_{f}=\alpha(1-\alpha)\left(\psi_{P}+\psi_{N}\right)
$$

\subsubsection{Gradient terms}

In an analogue way to the divergence terms, the integral of the gradient terms over a control volume is discretized as

$$
\int_{\Omega} \nabla \Psi d V=\int_{\partial \Omega} \Psi d S \approx \sum_{f} \Psi_{f} \bar{S}_{f}
$$

where $\Psi$ represents a generic variable.

Similarly to equation (3.9), the gradient term can be finally expressed as

$$
\sum_{f} \Psi_{f} \bar{S}_{f}=\sum_{f}\left[\alpha \bar{S}_{f} \Psi_{P f}+(1-\alpha) \bar{S}_{f} \Psi_{N f}\right]
$$




\subsubsection{Convection differencing scheme}

As shown in the previous subsections, the values of the different variables at the face between two cells are required to form the different convective terms, for which a differencing scheme needs to be selected. The scheme plays a significant role in the whole solution stability and accuracy. Furthermore, since we make use of unstructured meshes, it would be impractical to use differencing schemes that would require information from any other cell other than the ones that share the face.

The simplest case is to assign to each face the value of the corresponding cell, resulting in a bounded first order accurate scheme. If second order spatial accuracy were required, the value at the face is interpolated linearly between the cell centers; unfortunately, as proven by Godunov's theorem (Wesseling, 2009), this produces unphysical oscillations that seriously jeopardize the stability of the solution, making necessary the use of slope limiters (see $\S 6.1 .2$ ).

\subsection{Diffusive terms}

The diffusive terms in equations (3.2)-(3.3) are given by $\nabla \cdot \mathcal{T}, \nabla \cdot(\mathcal{T} \cdot \bar{u})$ and $\nabla \cdot\left(k_{e} \nabla T\right)$ respectively. According to the definition of $\mathcal{T}$, the calculation of $\nabla(\bar{u})$ constitutes the first step. By making use of Gauss theorem, an approximation of the gradient of $\bar{u}$ is given by

$$
V_{P} \nabla \bar{u}_{P}=\sum_{f} \bar{u}_{f} \bar{S}_{f}^{T}
$$

and the value of the velocity at the cell faces is calculated by linear interpolation between the neighboring cells, such that

$$
\begin{aligned}
w_{f} & =\frac{\left|\bar{S}_{f} \cdot \overline{d_{f N}}\right|}{\left|\bar{S}_{f} \cdot \bar{d}\right|} \\
\bar{u}_{f} & =w_{f} \bar{u}_{P}+\left(1-w_{f}\right) \bar{u}_{N} .
\end{aligned}
$$

Upon calculation of $\mathcal{T}$, the same linear interpolation scheme is used to calculate the volume integral of $\nabla \cdot \mathcal{T}$, as

$$
\int_{\Omega} \nabla \cdot \mathcal{T} \approx \sum_{f} \mathcal{T} \cdot \bar{S}_{f}=\sum_{f}\left(w_{f} \mathcal{T}_{P}+\left(1-w_{f}\right) \mathcal{T}_{N}\right) \cdot \bar{S}_{f}
$$

In a similar way, making use of the same expression as in equation (3.9) we have

$$
\begin{aligned}
\int_{\Omega} \nabla \cdot(\mathcal{T} \cdot \bar{u}) & \approx \sum_{f}\left(\mathcal{T} \cdot \bar{u}_{f}\right) \cdot \bar{S}_{f}= \\
& =\sum_{f}\left[\left(w_{f} \mathcal{T}_{P}+\left(1-w_{f}\right) \mathcal{T}_{N}\right) \cdot\left(\alpha \bar{u}_{P}+(1-\alpha) \bar{u}_{N}\right)\right] \cdot \bar{S}_{f}
\end{aligned}
$$


Finally, the thermal conductivity term is approximated by a first order interpolation, as

$$
\int_{\Omega} \nabla \cdot\left(k_{e} \nabla T\right) \approx \sum_{f} k_{e_{f}}(\nabla T)_{f} \cdot \bar{S}_{f} \approx \sum_{f} k_{e_{f}} \frac{\left|\bar{S}_{f}\right|^{2}}{\bar{S}_{f} \cdot \bar{d}}\left(T_{N}-T_{P}\right)
$$

The diffusive terms (see equation (3.4)) can also be decomposed into a Laplacian term and a term that depends on the gradient and its transpose. The gradient dependent term is treated as shown above. The Laplacian term can then be treated implicitly, in order to improve the stability of the numerical scheme, by discretizing it as

$$
\int_{\Omega} \nabla \cdot(\Gamma \nabla \Psi) d V \approx \sum_{f} \Gamma_{f} \bar{S}_{f} \cdot(\nabla \Psi)_{f}
$$

where $\Gamma$ is the diffusion coefficient. $\Gamma_{f}$ will be interpolated linearly from the cell center values. For the general case in which $\bar{S}_{f}$ is not parallel to $\bar{d}$, the evaluation of $\bar{S}_{f} \cdot(\nabla \Psi)_{f}$ is split (Jasak, 1996) into an orthogonal component and a non-orthogonal correction, as

$$
\bar{S}_{f} \cdot(\nabla \Psi)_{f}=\underbrace{\frac{\left|\bar{S}_{f}\right|^{2}}{\bar{S}_{f} \cdot \bar{d}}\left(\Psi_{N}-\Psi_{P}\right)}_{\text {orthogonal }}+\underbrace{\left[\bar{S}_{f}-\frac{\left|\bar{S}_{f}\right|^{2} \cdot \bar{d}}{\bar{S}_{f} \cdot \bar{d}}\right] \cdot(\nabla \Psi)_{f}}_{\text {non-orthogonal correction }},
$$

where the orthogonal part can be easily treated implicitly if required during the solution. Since the fluid equations are evolved in time making use of sub-iterations, the non-orthogonal correction is treated explicitly with the values from the previous subiteration, i.e. no orthogonal correction iterative loop is added to the solution scheme.

\subsection{Scaling and non-dimensionalization}

Upon closer inspection of the fluid governing equations, and both the conserved and primitive variables, it is clear that some of them differ from the others by orders of magnitude. In implementing a POD based ROM, this would mean that the majority of the modes tend to focus on minimizing the RMS error of the largest variables. In order to prevent this, the equations are rewritten in non-dimensional form and scaled adequately. Additionally, non-dimensionalized equations help to gain a greater insight into the relative size of various terms present in the equation

Following (Pulliam, 1986), considering $\infty$ as a reference to free stream quantities, the time variable is scaled as

$$
\tilde{t}=t \frac{a_{\infty}}{l}
$$

where the overtilde denotes a non-dimensional variable; $a_{\infty}$ is the speed of sound ( $a=$ $\sqrt{\gamma p / \rho}$ for ideal fluids) and $l$ is a reference length, e.g. the chord in an airfoil. In a 
similar way, the remaining variables are non-dimensionalized as:

$$
\begin{array}{rlrl}
\tilde{\rho} & =\frac{\rho}{\rho_{\infty}}, \\
\tilde{u}=\frac{u}{a_{\infty}}, & \tilde{v} & =\frac{v}{a_{\infty}}, \quad \tilde{w}=\frac{w}{a_{\infty}}, \\
\tilde{e}=\frac{e}{\rho_{\infty} a_{\infty}^{2}}, & \tilde{p} & =\frac{p}{\rho_{\infty} a_{\infty}^{2}}, \quad \tilde{T}=\frac{\gamma \tilde{p}}{\tilde{\rho}}=\left(\frac{a}{a_{\infty}}\right)^{2} .
\end{array}
$$

The molecular viscosity is defined from Sutherland's law (Sutherland, 1893) as

$$
\tilde{\mu}=\frac{\mu}{\mu_{\infty}}=\tilde{T}^{\frac{3}{2}}\left[\frac{1+\frac{\tilde{c}}{T_{\infty}}}{\tilde{T}+\frac{\tilde{c}}{T_{\infty}}}\right],
$$

where $\tilde{c}=110.4 \mathrm{~K}$ is the Sutherland's constant. Similarly, making use of the Prandtl number we have that

$$
\tilde{k}=\frac{\tilde{\mu}}{\operatorname{Pr}(\gamma-1)} .
$$

If the non-dimensional relations are substituted in equations (3.1)-(3.3) and the different terms rearranged, it is straight forward to see that they remain unchanged, with the exception of the effective fluid viscosity $\mu_{e}$ and the effective fluid conductivity $k_{e}$. If we define the Reynolds number, based on the free stream velocity, as $R e=\rho_{\infty} l u_{\infty} / \mu_{\infty}$, they become

$$
\tilde{\mu}_{e} \rightarrow \frac{M_{\infty}}{R e} \tilde{\mu}_{e}, \quad \tilde{k}_{e} \rightarrow \frac{M_{\infty} \tilde{k}_{e}}{R e} .
$$

In the remaining of this work, non-dimensional variables will be used, and overtildes dropped for simplicity.

\subsection{Boundary conditions}

The set of equations require boundary conditions on all sides of the domain, as well as on all surfaces which lie within the domain. In general, it is required to fix the value at the boundary (Dirichlet) or impose a given gradient at the boundary (Neumann). The fluid solver works with the face values of the primitive variables and their gradients, which are assigned for each physical boundary condition as follows in the next subsections. Note that the boundary conditions are imposed on the faces prior to the gradient calculations, so the gradients already account for the imposed face value by default.

\section{Subsonic inlet}

For a subsonic inlet boundary condition the free stream values are set for the density and the velocities, and zero gradient is imposed for the pressure, so that if $b$ is the 
boundary face, we have

$$
\begin{gathered}
\rho_{P b}=\rho_{N b}=\rho_{b}=1.0 \\
u_{P b}=u_{N b}=u_{b}=M_{\infty} \cos \left(\alpha_{\infty}\right) \cos \left(\beta_{\infty}\right), \\
v_{P b}=v_{N b}=v_{b}=M_{\infty} \sin \left(\alpha_{\infty}\right) \cos \left(\beta_{\infty}\right) \\
w_{P b}=w_{N b}=w_{b}=-M_{\infty} \sin \left(\beta_{\infty}\right) \\
p_{P b}=p_{N b}=p_{b}=p_{P} \\
\nabla p_{b}=0
\end{gathered}
$$

\section{Subsonic outlet}

For a subsonic outlet boundary condition the free stream values are set for the pressure, and zero gradient is imposed for the density and the velocities, so that if $b$ is the boundary face, we have

$$
\begin{gathered}
\rho_{P b}=\rho_{N b}=\rho_{b}=\rho_{P}, \\
u_{P b}=u_{N b}=u_{b}=u_{P}, \\
v_{P b}=v_{N b}=v_{b}=v_{P}, \\
w_{P b}=w_{N b}=w_{b}=w_{P}, \\
p_{P b}=p_{N b}=p_{b}=\frac{1}{\gamma}, \\
\nabla \rho_{b}=\nabla u_{b}=\nabla v_{b}=\nabla w_{b}=0 .
\end{gathered}
$$

\section{Subsonic inlet/outlet}

A generic subsonic inlet/outlet boundary condition can be imposed in the farfield making use of Riemann invariants. The final solution is a very stable and robust farfield boundary condition. This boundary condition has been implemented similarly as in FUN3D and CFL3D (Bartels et al., 2006; Carlson, 2011).

The Riemann invariants correspond to the incoming $R^{-}$and outgoing $R^{+}$characteristic waves, and they are given by

$$
R^{+}=U_{P}+\frac{2\left(T_{P}\right)^{\frac{1}{2}}}{\gamma-1}, \quad R^{-}=U_{\infty}-\frac{2}{\gamma-1}
$$

where

$$
U_{P}=\bar{u}_{P} \cdot \bar{n}, \quad U_{\infty}=\bar{u}_{\infty} \cdot \bar{n} .
$$

The normal velocity, $U_{b}$, and the speed of sound, $a_{b}$, at the boundary face $b$ are the sum and difference of the invariants:

$$
U_{b}=\frac{R^{+}+R^{-}}{2}, \quad a_{b}=\frac{\gamma-1}{4}\left(R^{+}-R^{-}\right) .
$$


The velocities are determined by decomposing the normal and tangential velocity vectors. The sign of $U_{b}$ determines whether the condition is at inflow $\left(U_{b}<0\right)$ or outflow $\left(U_{b}>0\right)$. For an inflow condition the entropy $\left(S_{b}\right)$ and the velocities are determined using the values from outside the domain. The values from inside the domain are used in the case of an outflow condition. Summarizing, we have:

- Inflow $\left(U_{b}<0\right)$

$$
\begin{gathered}
\bar{u}_{P b}=\bar{u}_{N b}=\bar{u}_{b}=\bar{u}_{\infty}+\left(U_{b}-U_{\infty}\right) \cdot \bar{n} \\
S_{b}=\frac{1}{\gamma} .
\end{gathered}
$$

- Outflow $\left(U_{b}>0\right)$

$$
\begin{gathered}
\bar{u}_{P b}=\bar{u}_{N b}=\bar{u}_{b}=\bar{u}_{P}+\left(U_{b}-U_{P}\right) \cdot \bar{n}, \\
S_{b}=\frac{T_{P}}{\gamma \rho_{P}^{\gamma-1}} .
\end{gathered}
$$

Finally, the fluid state variables are recovered as

$$
\begin{gathered}
\rho_{P b}=\rho_{N b}=\rho_{b}=\left(\frac{a_{b}^{2}}{\gamma S_{b}}\right)^{\frac{1}{\gamma-1}}, \\
p_{P b}=p_{N b}=p_{b}=\frac{\rho_{b} a_{b}^{2}}{\gamma} .
\end{gathered}
$$

\section{Slip wall}

For a slip wall, the flow normal to the wall needs to be null. The density and pressure values at the face are approximated with the nearest cell center point on the grid.

If the flow normal to the wall face $b$ is defined as

$$
\phi_{b}=\left(\bar{u}_{P}-\overline{\mathrm{w}}_{b}\right) \cdot \bar{n},
$$

where $\bar{w}_{b}$ accounts for the wall motion, we have that the fluid velocity at the face is determined by

$$
\bar{u}_{P b}=\bar{u}_{N b}=\bar{u}_{b}=\bar{u}_{P}-\left(\phi_{b}\right) \bar{n} .
$$

Finally, the remaining variables are set as

$$
\begin{aligned}
& \rho_{P b}=\rho_{N b}=\rho_{b}=\rho_{P}, \\
& p_{P b}=p_{N b}=p_{b}=p_{P} .
\end{aligned}
$$

\section{No-slip adiabatic wall}

For an adiabatic no-slip wall boundary condition, the relative velocity between the fluid and the body is required to be null. Additionally, no heat transfer occurs at the wall. 
The density and pressure values at the face are approximated with the nearest cell center point on the grid, which for the typical fine meshes used in practical applications is deemed to be a reasonable approximation.

The boundary condition is enforced as:

$$
\begin{gathered}
\rho_{P b}=\rho_{N b}=\rho_{b}=\rho_{P}, \\
p_{P b}=p_{N b}=p_{b}=p_{P}, \\
T_{P b}=T_{N b}=T_{b}=T_{P}\left[1+\frac{\gamma-1}{2}\left(u_{P}^{2}+v_{P}^{2}+w_{P}^{2}\right)\right] \\
\rho_{P b}=\rho_{N b}=\rho_{b}=\frac{\gamma p_{b}}{T_{b}} \\
\bar{u}_{P b}=\bar{u}_{N b}=\bar{u}_{b}=\overline{\mathrm{w}}_{b} .
\end{gathered}
$$

\subsection{Primitive and conserved variables}

In the previous sections two set of flow variables have been presented, the conserved variables $\mathrm{q}=(\rho, \rho u, \rho v, \rho w, \rho E)$ and the primitive variables $\mathrm{v}=(\rho, u, v, w, p)$. For practical reasons in the creation of the POD based modes, it is preferable to work with the primitive variables. Since the flow governing equations are expressed in the conserved variables, a transformation is required. This is achieved by expressing

$$
M=\frac{\partial \mathrm{q}}{\partial \mathrm{v}}=\left[\begin{array}{ccccc}
1 & 0 & 0 & 0 & 0 \\
u & \rho & 0 & 0 & 0 \\
v & 0 & \rho & 0 & 0 \\
w & 0 & 0 & \rho & 0 \\
\frac{u^{2}+v^{2}+w^{2}}{2} & \rho u & \rho v & \rho w & \frac{1}{\gamma-1}
\end{array}\right]
$$

so that, using the derivative chain rule, we have

$$
\frac{\partial \mathrm{v}}{\partial t}=\frac{\partial \mathrm{v}}{\partial \mathrm{q}} \frac{\partial \mathrm{q}}{\partial t}=M^{-1} \frac{\partial \mathrm{q}}{\partial t}
$$




\section{Chapter 4}

\section{Fluid structure interaction}

One key aspect of the present work is the new extension of the POD on the fly ROM to unsteady fluid structure interaction problems. To close the problem definition, it is necessary to focus on the structural model, as well as the interaction between the fluid and the structure.

\subsection{Structural model}

The foundation of aeroelasticity lays on the conditions of equilibrium and compatibility of an elastic deformable body, together with appropriate force and displacement boundary conditions (Bisplinghoff et al., 1955). The structural model describes the motion and deformation of the body under the given external and internal forces.

\subsubsection{Lagrangian mechanics}

The motion of the structural dynamical system is completely described by a set of independent generalized coordinates. In other words, the displacement of a point in the body can be written as

$$
\vec{r}=\vec{r}\left(x_{1}, x_{2}, \ldots, x_{N}\right) .
$$

According to Hamilton's principle in variational form applied to deformable bodies (Dowell, Crawley, et al., 1995)

$$
\int_{t_{o}}^{t_{1}}\left[\delta(\mathrm{T}-\mathrm{U})+\delta \mathrm{W}_{\mathrm{NC}}\right] d t=0
$$

where $\mathrm{T}$ is the body kinetic energy, $\mathrm{U}$ is the body potential energy, and $\delta \mathrm{W}_{\mathrm{NC}}$ represents the work performed by the external and non-conservative forces. This expression can be rewritten as

$$
\sum_{i} \int_{t_{o}}^{t_{1}}\left[\frac{\partial(\mathrm{T}-\mathrm{U})}{\partial \dot{x}_{i}} \delta \dot{x}_{i}+\frac{\partial(\mathrm{T}-\mathrm{U})}{\partial x_{i}} \delta x_{i}+\mathcal{F}_{i} \delta x_{i}\right] d t=0
$$


where, $\mathcal{F}_{i}$ represents the generalized forces, given by

$$
\mathcal{F}_{i}=F \frac{\partial \vec{r}}{\partial x_{i}} \delta x_{i}
$$

In the particular case of a body immersed in a fluid, the generalized forces are

$$
\mathcal{F}_{i}=\int_{\partial \Omega_{b o d y}} p \frac{\partial \vec{r}}{\partial x_{i}} \delta x_{i} d \bar{S}-\int_{\partial \Omega_{b o d y}}\left(\mu_{e} \frac{\partial \overline{u_{t}}}{\partial n} \bar{t}\right) \frac{\partial \vec{r}}{\partial x_{i}} \delta x_{i} d S
$$

where $\overline{u_{t}}$ represents the tangential velocity vector (which vanishes on the surface), $\bar{S}$ is the surface normal vector pointing out of the fluid, $n$ is a coordinate along the unit normal to the surface, and $\bar{t}$ is a vector tangential to the surface in the direction of the flow. The second term represents the friction forces on the body, which are traditionally neglected in many aeroelastic applications.

Integrating by parts the first term of equation (4.3), and noting that $\delta x_{i}=0$ at $t=t_{o}$ and $t=t_{1}$ because the state of the system is specified at these instants, we have

$$
\left.\sum_{i} \frac{\partial(\mathrm{T}-\mathrm{U})}{\partial x_{i}} \delta x_{i}\right|_{t_{o}} ^{t_{1}}+\int_{t_{o}}^{t_{1}}\left[-\frac{d}{d t} \frac{\partial(\mathrm{T}-\mathrm{U})}{\partial \dot{x}_{i}}+\frac{\partial(\mathrm{T}-\mathrm{U})}{\partial x_{i}}+\mathcal{F}_{i}\right] \delta x_{i}=0
$$

Since this equation must hold for all $\delta x_{i}$, it follows that each bracketed quantity must be zero, and the Lagrange equations are obtained as

$$
-\frac{d}{d t} \frac{\partial(\mathrm{T}-\mathrm{U})}{\partial \dot{x}_{i}}+\frac{\partial(\mathrm{T}-\mathrm{U})}{\partial x_{i}}+\mathcal{F}_{i}=0 \quad i=1,2 \ldots
$$

\subsubsection{Example: Airfoil with control surface}

Let us consider the three degrees of freedom (DoF) airfoil sketched in figure 4.1, which can heave and rotate around the center of rotation, located at a distance $x_{\text {rot }}$ from the nose. A control surface, attached to it, can rotate around a hinge placed at a distance $x_{c}$ from the center of rotation. Its motion can subsequently be described with three generalized coordinates, namely, the vertical deflection $h$, the rotation around the center of rotation $\theta$ and the control surface deflection angle $\beta$.

The system mass properties can be represented by the box mass $\left(M_{B}\right)$, the position of the box center of gravity $(\mathrm{CG})$ relative to the rotation center $\left(x_{B}\right)$, the mass inertia of the box around its CG $\left(I_{B}\right)$, the control surface mass $\left(M_{C s}\right)$, the position of the control surface $C G$ relative to the hinge $\left(x_{c s}\right)$, and the mass inertia of the control surface around its CG $\left(I_{c s}\right)$.

Invoking the Lagrange approach to describe the system dynamics, assuming small control surface rotations, the various ingredients in the governing equations are as follows. 


\section{Kinetic energy}

The kinetic energy of the system can be expressed as

$$
\mathrm{T}=\frac{1}{2} I_{B} \dot{\theta^{2}}+\frac{1}{2} I_{c s}(\dot{\theta}+\dot{\beta})^{2}+\frac{1}{2} M_{B} V_{B}^{2}+\frac{1}{2} M_{c s} V_{c s}^{2},
$$

with

$$
\begin{aligned}
V_{B} & =\dot{h}+x_{B} \dot{\theta} \\
V_{c s} & =\dot{h}+\left(x_{c}+x_{c s}\right) \dot{\theta}+x_{c s} \dot{\beta},
\end{aligned}
$$

Combining all the terms, and defining

$$
\begin{aligned}
M & =M_{B}+M_{c s} \\
I_{\theta} & =I_{B}+I_{c s}+M_{B} x_{B}^{2}+M_{c s}\left(x_{c}+x_{c s}\right)^{2} \\
x_{\theta} & =\frac{M_{B} x_{B}+M_{c s}\left(x_{c}+x_{c s}\right)}{M} \\
I_{\beta} & =I_{c s}+M_{c s} x_{c s}^{2}
\end{aligned}
$$

as the total mass of the system, the total inertia of the system around the rotation center, the location of the system CG and the inertia of the control surface around the hinge, respectively, the kinetic energy is written as

$$
\mathrm{T}=\frac{1}{2} M \dot{h}^{2}+\frac{1}{2} I_{\theta} \dot{\theta}^{2}+\frac{1}{2} I_{\beta} \dot{\beta}^{2}+M x_{\theta} \dot{h} \dot{\theta}+M_{c s} x_{c s} \dot{h} \dot{\beta}+\left[x_{c} x_{c s} M_{\beta}+I_{\beta}\right] \dot{\theta} \dot{\beta} .
$$

Potential energy

$$
\mathrm{U}=\frac{1}{2} M \omega_{h} h^{2}+\frac{1}{2} I_{\theta} \omega_{\theta} \theta^{2}+\frac{1}{2} I_{\beta} \omega_{\beta} \beta^{2}
$$

where

$$
\omega_{h}^{2}=\frac{k_{h}}{M} ; \quad \omega_{\theta}^{2}=\frac{k_{\theta}}{I_{\theta}} ; \quad \omega_{\beta}^{2}=\frac{k_{\beta}}{I_{\beta}}
$$

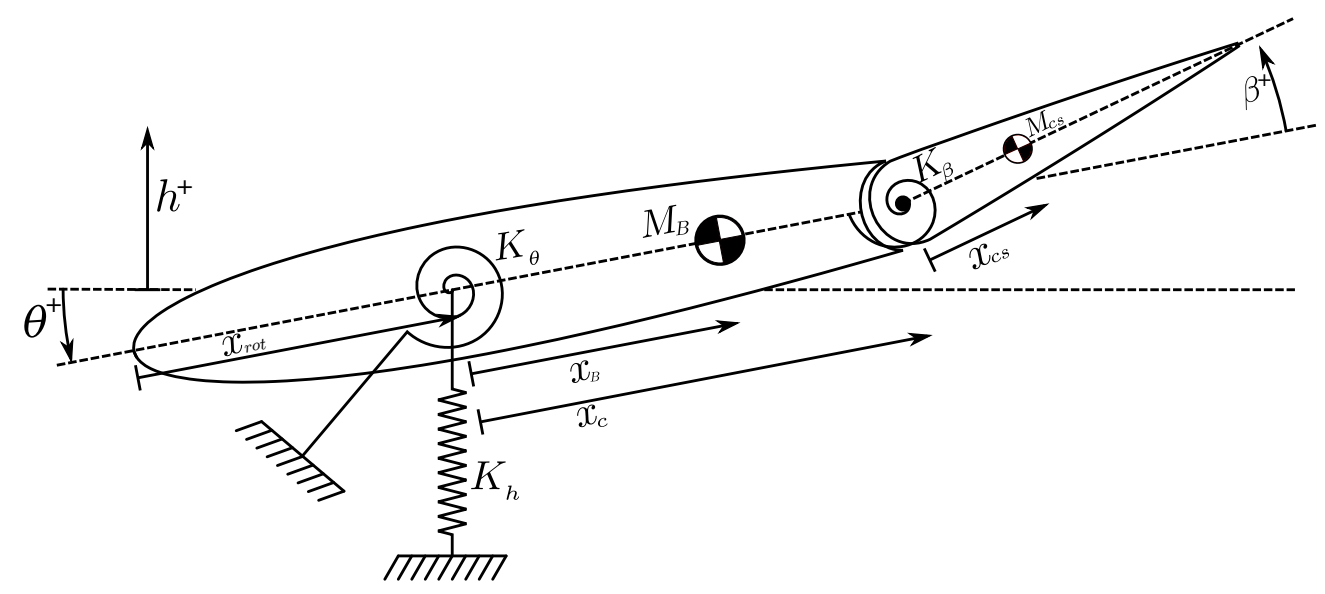

Figure 4.1: Airfoil description 
correspond to the uncoupled eigenfrequencies, i.e. those corresponding to the modes in which only the given degree of freedom is free.

\section{Energy dissipation}

If a structural damping formulation is used, which is in fact the most common approach in the industry, the energy dissipation is given by

$$
D=\frac{1}{2} M g_{h} \omega_{h} \dot{h}^{2}+\frac{1}{2} I_{\theta} g_{\theta} \omega_{\theta} \dot{\theta}^{2}+\frac{1}{2} I_{\beta} g_{\beta} \omega_{\beta} \dot{\beta}^{2}
$$

where $g_{h}, g_{\theta}$ and $g_{\beta}$ are the structural damping factors corresponding to the heave, pitch and control surface rotation respectively. Despite the fact that this formulation is justified only for harmonic motions, its use for non-harmonic motions is commonly accepted.

\section{Governing Equations}

Applying the Lagrange equations, namely

$$
\frac{d}{d t}\left(\frac{\partial T}{\partial \dot{x_{i}}}\right)+\frac{\partial U}{\partial x_{i}}+\frac{\partial D}{\partial \dot{x_{i}}}=\mathcal{F}_{i}
$$

the governing equations of the airfoil motion are:

$$
\begin{gathered}
\ddot{h}+x_{\theta} \ddot{\theta}+\frac{M_{c s}}{M} x_{c s} \ddot{\beta}+g_{h} \omega_{h}^{2} \dot{h}+\omega_{h}^{2} h=\frac{\mathcal{F}_{h}}{M}=-\frac{1}{M} \int_{\partial \Omega} p S_{f z} d s \\
x_{\theta} \ddot{h}+\frac{I_{\theta}}{M} \ddot{\theta}+\left[\frac{M_{c s}}{M} x_{c} x_{c s}+\frac{I_{\beta}}{M}\right] \ddot{\beta}+\frac{I_{\theta}}{M} g_{\theta} \omega_{\theta}^{2} \dot{\theta}+\frac{I_{\theta}}{M} \omega_{\theta}^{2} \theta=\frac{\mathcal{F}_{\theta}}{M}=-\frac{1}{M} \int_{\partial \Omega} p x S_{f z} d s \\
\frac{M_{c s}}{M} x_{c s} \ddot{h}+\left[\frac{M_{c s}}{M} x_{c} x_{c s}+\frac{I_{\beta}}{M}\right] \ddot{\theta}+\frac{I_{\beta}}{M} \ddot{\beta}+\frac{I_{\beta}}{M} g_{\beta} \omega_{\beta}^{2} \dot{\beta}+\frac{I_{\beta}}{M} \omega_{\beta}^{2} \beta=\frac{\mathcal{F}_{\beta}}{M}= \\
=-\frac{1}{M} \int_{\partial \Omega_{c s}} p\left(x-x_{c}\right) S_{f z} d s
\end{gathered}
$$

In order to integrate these equations in time, it is more convenient to express them in matrix form as

$$
\mathcal{M} \frac{d \tilde{x}}{d t}+\mathcal{L} \tilde{x}=\mathcal{F}
$$

with

$$
\begin{gathered}
\tilde{x}(t)=[\dot{h}, \dot{\theta}, \dot{\beta}, h, \theta, \beta]^{T}, \\
\mathcal{F}=\left[\frac{\mathcal{F}_{h}}{M}, \frac{\mathcal{F}_{\theta}}{M}, \frac{\mathcal{F}_{\beta}}{M}, 0,0,0\right]^{T},
\end{gathered}
$$




$$
\begin{aligned}
& \mathcal{M}=\left[\begin{array}{cccccc}
1 & x_{\alpha} & x_{c s} \frac{M_{c s}}{M} & 0 & 0 & 0 \\
x_{\alpha} & \frac{I_{\theta}}{M} & \frac{M_{c s}}{M} x_{c} x_{c s}+\frac{I_{\beta}}{M} & 0 & 0 & 0 \\
x_{c s} \frac{M_{c s}}{M} & \frac{M_{c s}}{M} x_{c} x_{c s}+\frac{I_{\beta}}{M} & \frac{I_{\beta}}{M} & 0 & 0 & 0 \\
0 & 0 & 0 & 1 & 0 & 0 \\
0 & 0 & 0 & 0 & 1 & 0 \\
0 & 0 & 0 & 0 & 0 & 1
\end{array}\right] \\
& \mathcal{L}=\left[\begin{array}{cccccc}
\omega_{h}^{2} g_{h} & 0 & 0 & \omega_{h}^{2} & 0 & 0 \\
0 & \frac{I_{\theta}}{M} g_{\theta} \omega_{\theta}^{2} & 0 & 0 & \frac{I_{\theta}}{M} \omega_{\theta}^{2} & 0 \\
0 & 0 & \frac{I_{\beta}}{M} g_{\beta} \omega_{\beta}^{2} & 0 & 0 & \frac{I_{\beta}}{M} \omega_{\beta}^{2} \\
-1 & 0 & 0 & 0 & 0 & 0 \\
0 & -1 & 0 & 0 & 0 & 0 \\
0 & 0 & -1 & 0 & 0 & 0
\end{array}\right]
\end{aligned}
$$

\section{$4.2 \quad$ Fluid-structure coupling}

In the previous section, it was shown how the forces generated by the fluid on the bodyfluid interface participate in the dynamic motion of the structure. The body interacts with the fluid through the motion of the interface and the imposition of the appropriate boundary conditions.

\subsubsection{Rigid body}

In the particular case in which the body is rigid, that is, when the distance between any two given points of the body remains constant in time, the numerical fluid domain moves rigidly together with the body. In other words, the relative distance between any two given points in the numerical mesh, and the volume of the cells, remain constant in time

If we consider two sets of coordinate axes, one that is fixed to the rigid body and is allowed to rotate following the body motion, and other that is fixed in space, the position of a given cell point $P$ (see figure 4.2) in the fluid domain is given by

$$
r^{\prime}=R+r
$$

Subsequently, the velocity is given by (Marion, 2013)

$$
\left(\frac{d r^{\prime}}{d t}\right)_{\text {fixed }}=\left(\frac{d R}{d t}\right)_{\text {fixed }}+\left(\frac{d r}{d t}\right)_{\text {rotating }}+\omega \times r
$$

In this way, the velocity of the control volume face specified in equations (3.1)-(3.3) is easily obtained. 


\subsubsection{Deformable body}

For generic deformable bodies, the simplest of which might be formed by two linked rigid bodies allowed to have relative motion, a new ingredient is required. The numerical fluid domain mesh needs to move in such a way that it is conformal to the structure. Additionally, the degradation of the mesh quality needs to be as small as possible, since it could introduce errors in the solution.

The explicit interpolation method developed by Luke and Blades (Luke et al., 2012) has been chosen in the present work due to its robustness, improved quality of the deformed mesh, efficiency and reduced numerical cost. In this method, the deformation of the volume mesh is given as a weighted sum of the rigid body displacements corresponding to each body-fluid interface surface point. If node $i$ is on the deforming surface, its displacement field $\vec{S}_{i}(\vec{r})$ is given by

$$
\vec{S}_{i}(\vec{r})=M_{i}+\vec{b}_{i}-\vec{r}
$$

where $M_{i}$ is a rotation matrix, $\vec{b}_{i}$ is a displacement vector associated with the $i^{\text {th }}$ node, and $\vec{r}$ is a coordinate vector in the original mesh. The displacement field in the volume mesh is then described through a weighted average of all boundary node displacement fields, as

$$
\vec{S}(\vec{r})=\frac{\sum \mathrm{w}_{i}(\vec{r}) \vec{S}_{i}(\vec{r})}{\sum \mathrm{w}_{i}(\vec{r})}
$$

The interpolation weight function is chosen such that the weighting is a function of the inverse of distance. Using a two-exponent function, near-boundary deformations are

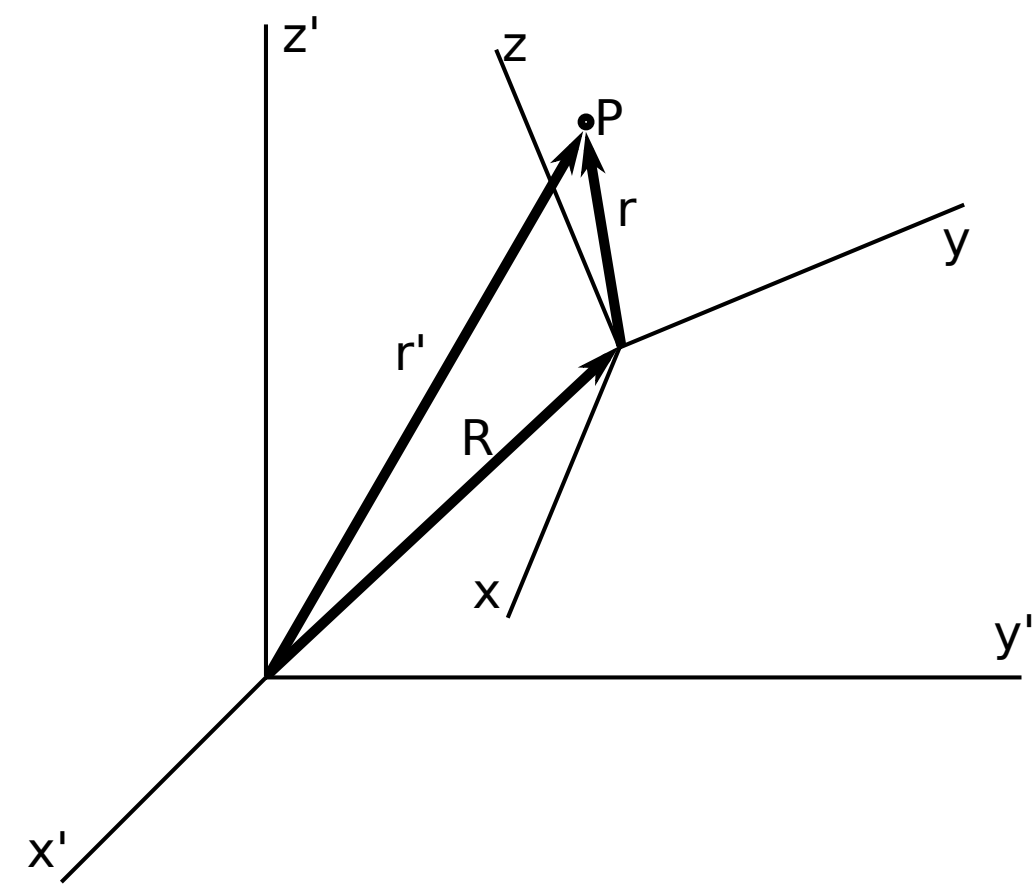

Figure 4.2: Non-inertial reference frame 
preserved while still providing a smooth transition. Finally, the area of the boundary face is used so that mesh refinement of a region (close to expected shocks, for example) does not increase its influence in the interpolation method. Taking the above into account, the resulting weight function is defined as

$$
\mathrm{w}_{i}(\vec{r})=\left|\bar{S}_{i}\right|\left[\left(\frac{L_{\text {def }}}{\left|\vec{r}-\overrightarrow{r_{i}}\right|}\right)^{a}+\left(\frac{\alpha L_{\text {def }}}{\left|\vec{r}-\overrightarrow{r_{i}}\right|}\right)^{b}\right]
$$

where $\mathrm{a}$ and $\mathrm{b}$ are user-defined tunable exponents, typically set to 3 and 5 respectively, $L_{d e f}$ is an estimated length of the deformation region, and $\alpha$ is an estimated size of the near body influence region, typically set to 0.2 to preserve viscous mesh quality in the boundary layer region.

One drawback to the method presented is the fact that the far-field cells, despite being very far from the body, are still deformed. This deformation is minimal but enough to affect the stability of the solution under certain circumstances. A simple modification has been introduced by including the far-field boundary faces in the calculation of the nodal displacement fields $\left(M_{i}\right.$ and $\vec{b}_{i}$ are null) and weights.

Figure 4.3 shows the application of the mesh deformation method to an airfoil with a control surface. As it can be seen, the orthogonality and the element size in the elements surrounding the airfoil are properly conserved.

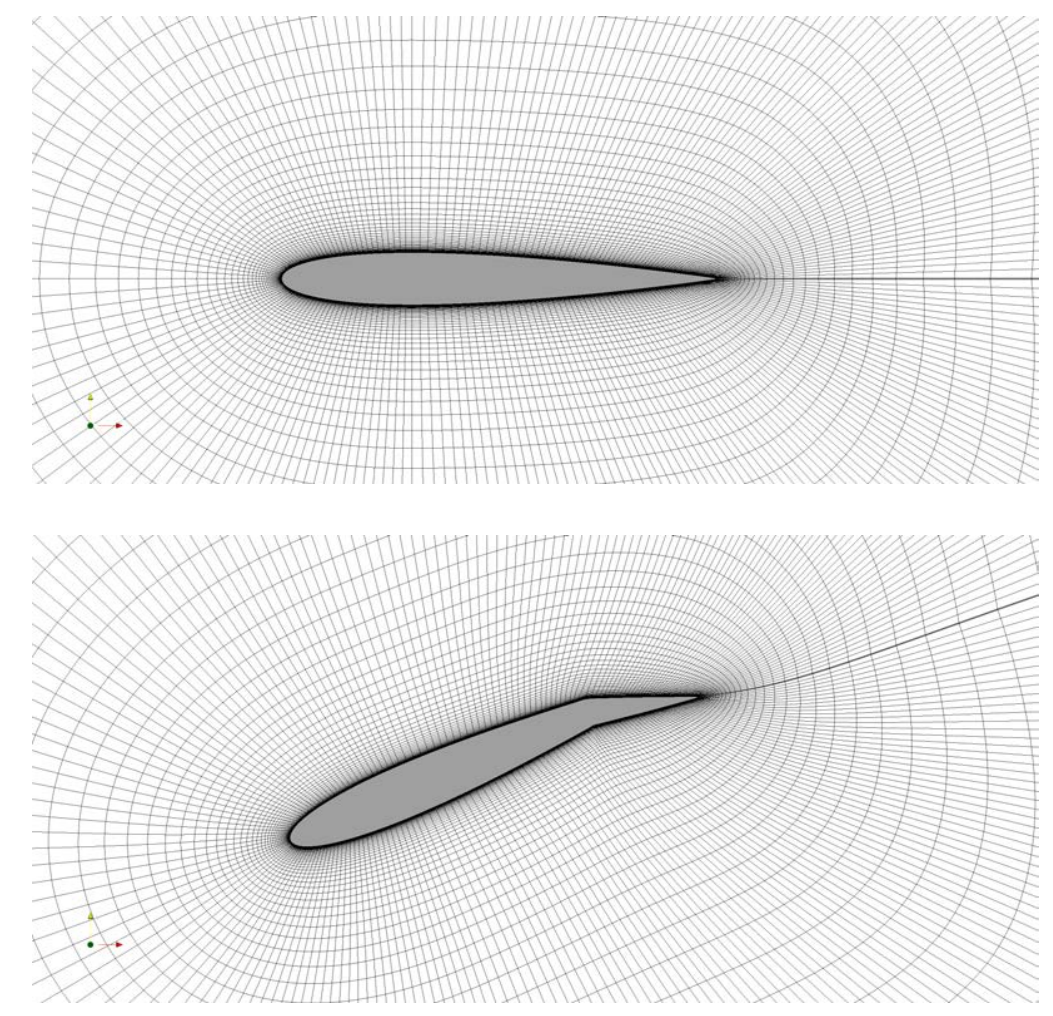

Figure 4.3: Mesh deformation example. Undeformed (top) and deformed (bottom) mesh using Luke and Blades deformation method 


\section{Chapter 5}

\section{Inviscid simulation application}

There are many fluid-structure coupling practical examples in the industry that could be used to show the benefits of applying the new methodology presented in the preceding chapters. The most common, and one that can result in very complex phenomena, is the interaction between a lifting surface and a control surface attached to it. The attachment stiffness between the control surface and the lifting surface typically includes some degree of non-linearity. Additionally, despite the tight manufacturing tolerances, the attachment is prone to exhibiting free-play regions. Furthermore, at certain angle of attack and Mach number combinations, the presence of the shock along the hinge line changes dramatically the behavior of the system.

Traditionally, in the pre-high-performance-computing era, such systems were studied by only retaining a wing section (see figure 5.1), usually the mean aerodynamic chord, so that the resulting system would be a three degrees of freedom (DoF) airfoil with heave, pitch and control surface rotation frequencies, namely the lifting surface first bending, first torsion and control surface rotation frequencies, respectively. Since analytical, potential based and linear, expressions exist (see Appendix B) for the aerodynamic forces acting on such an airfoil (Theodorsen, 1935), the analyses could be solved retaining at the same time all significant contributing factors to the phenomenom.

In the interest of showing the application of the method presented before, and in order to reduce the cost of the simulations, a similar approach has been taken in the present work by studying a three DoF airfoil. In the absence of potential based aerodynamics, the computational cost is lowered dramatically by reducing the 3D problem to a $2 \mathrm{D}$ problem, although the method is expected to show greater acceleration factors in the three-dimensional case. For the same reason, viscosity has been initially neglected in the computational fluid dynamics solver, avoiding the expensive calculation of the stress tensor. Neglecting the viscous terms might be seen as a significant simplification, but in reality the absence of dissipation in the fluid dynamics poses an additional challenge to the simulation stability.

For the reasons presented above, the current selection of the 3 DoF airfoil immersed in an inviscid flow at subsonic and transonic regimes is deemed as an adequate benchmark for the capabilities of the methods in a real industrial environment application. 
As an initial step, prior to performing any simulation, the CFD code has been validated for inviscid simulations (see Appendix C).

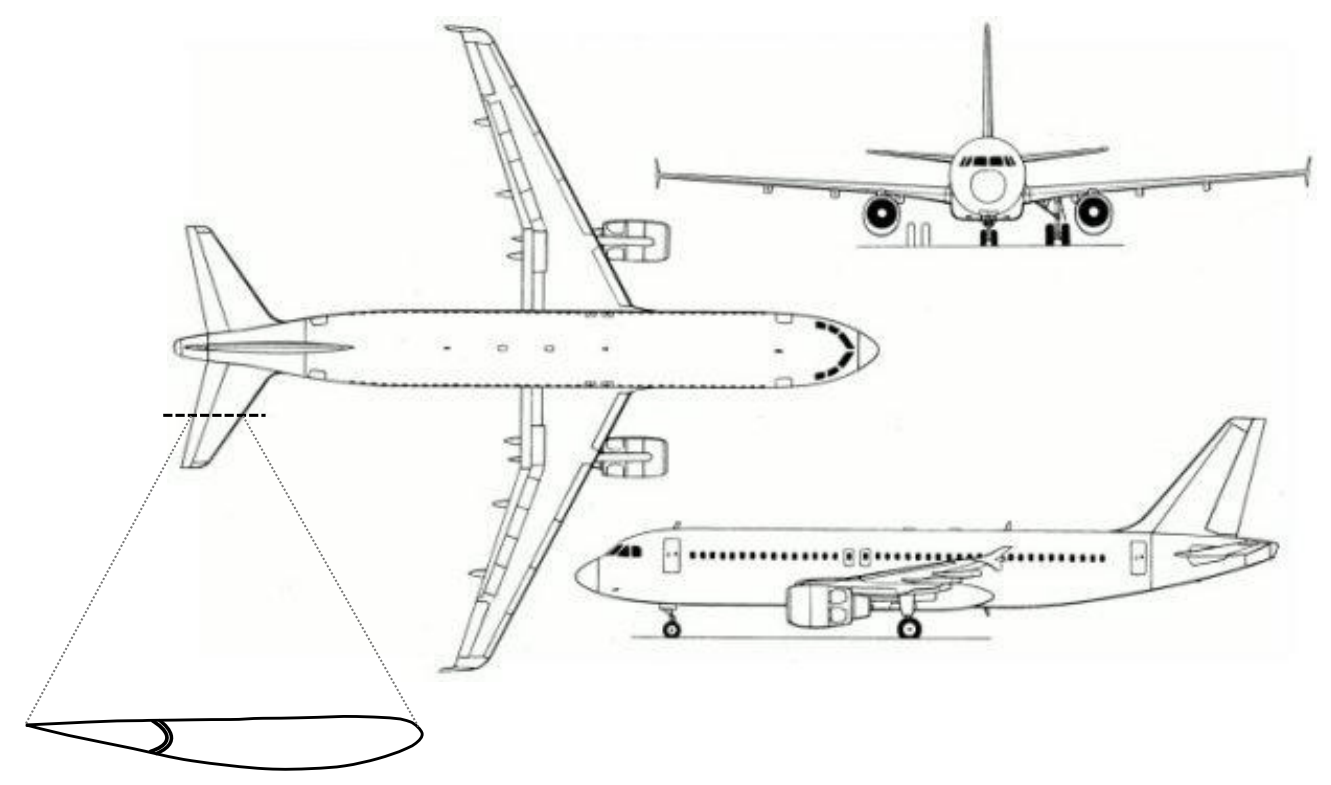

Figure 5.1: Horizontal tail section sketch

\subsection{Forced oscillation}

The prediction of the aerodynamic forces that an oscillating airfoil immersed in a fluid experiences is one of the key problems in unsteady aerodynamics, and a precursor to any computational dynamic aeroelasticity simulation. The analytical expressions for the aerodynamic forces acting on these airfoils, based on perturbation potential theory (Theodorsen, 1935), have made possible the prediction of instability boundaries of lifting surfaces in the origins of aeroelasticity. However, more precise simulations based on CFD are intended nowadays.

In the current example, the unsteady two-dimensional flow around an airfoil, subject to either pitch or heave oscillations, is considered in order to exercise the novel adaptive $\mathrm{ROM}$ approach presented in this thesis. This not only exercises the adaptive ROM, but also serves as a validation mean for the inviscid CFD solver.

In order to illustrate the benefits of the novel approach presented in this thesis, such an oscillating airfoil has been coupled to the inviscid flow solver previously described in Chapter 3.

\subsubsection{Computational fluid domain model}

The aerodynamic shape has been chosen, without loss of generality, as a 4 digits symmetric, uncambered, NACA airfoil with a $10 \%$ thickness to chord length ratio, known as NACA-0010 (Garrick, 1933). This type of airfoil (NACA 0008, NACA 0010 and NACA 
0012, in particular) has been commonly used in computational and experimental aeroelastic problems (Batina, 1985; Conner et al., 1997; Rivera et al., 1992).

The shape for the NACA 0010 airfoil is given by (Moran, 1984):

$$
y_{t}=0.5 c\left[0.2969 \sqrt{\frac{x}{c}}-0.1260 \frac{x}{c}-0.3516\left(\frac{x}{c}\right)^{2}+0.2843\left(\frac{x}{c}\right)^{3}-0.1015\left(\frac{x}{c}\right)^{4}\right] \text {, }
$$

where $c$ is the chord length, $x$ is the position along the chord (from 0 to $c$ ) and $y_{t}$ is the half thickness at a given value of $x$.

The fluid domain boundaries have been set as described in figure 5.2. A semi circular domain of radius 25 airfoil chords, with origin at $x=0.25 c$, defines the upstream boundary, and two 25 by 25 chords squares, whose corner has been smoothed to avoid numerical singularities during the mesh motion, define the upper, lower and downstream boundaries. Finally, the domain is extruded to a thickness of 1 chord.

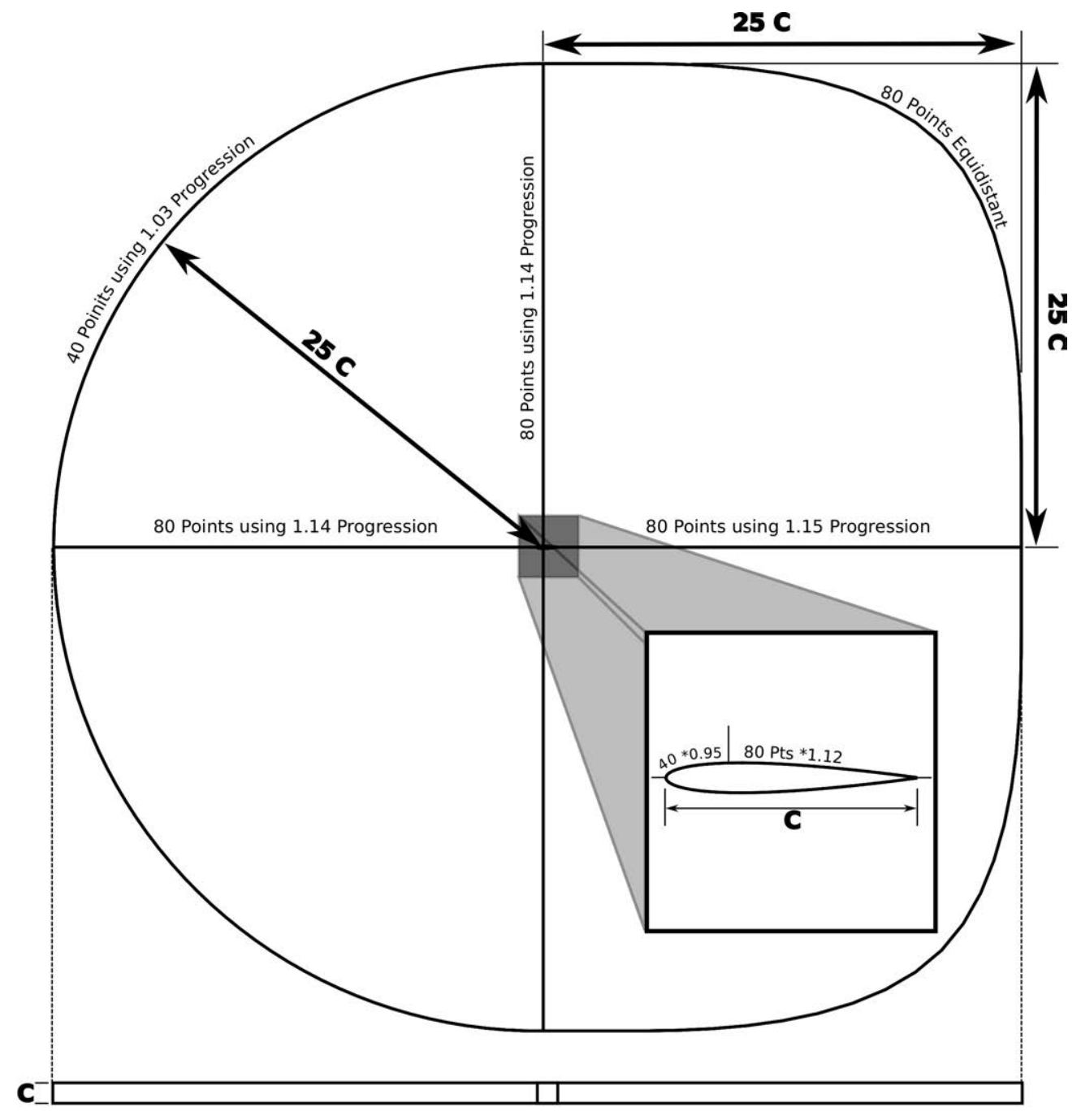

Figure 5.2: Airfoil mesh geometry 


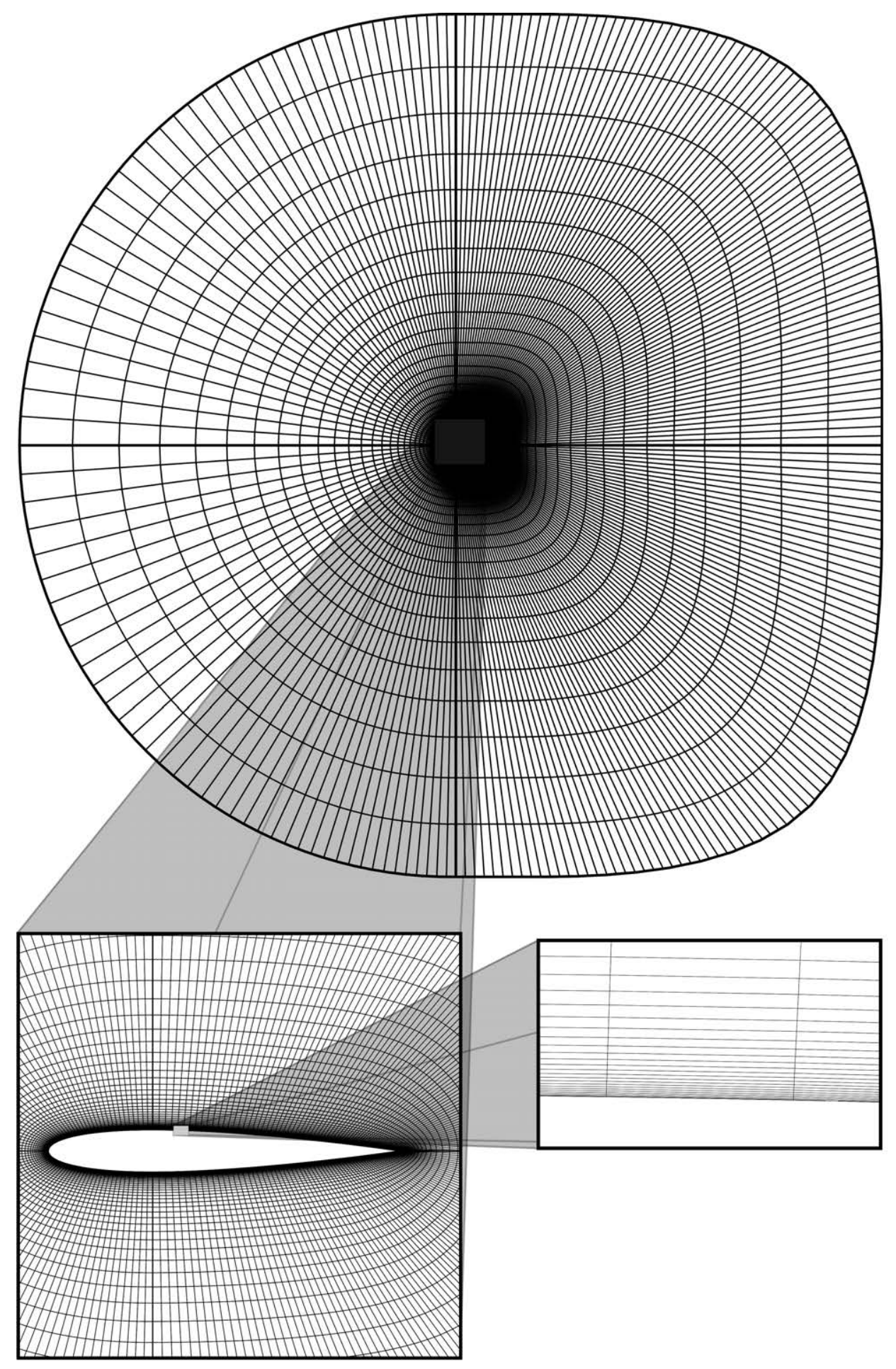

Figure 5.3: Airfoil mesh 
The computational fluid domain mesh has been generated in the boundary defined above using Gmsh (Geuzaine and Remacle, 2009), a three-dimensional finite element mesh generator with built-in pre- and post-processing utilities. First, a one-dimensional mesh seed is created along the boundaries (both internal and external); 40 points are distributed using a 0.95 distance growth rate in the two upstream quarters of the airfoil, and 80 points in the remaining two quarters (near the trailing edge), with 1.12 growth rate. Each of the far-field upstream quarters has 40 points, with 1.03 growth rate, and its radius includes 80 points with 1.14 growth rate. Finally, the downstream boundary and upper/lower boundaries include 80 equidistant points each.

In a second step, the two-dimensional mesh (see figure 5.3) is created using transfinite interpolation (Gordon and Hall, 1973).

Finally, since this two-dimensional problem is to be solved using a three-dimensional CFD solver, constructed as explained in Chapter 3, the two-dimensional mesh is extruded in the span direction to create the three-dimensional mesh consisting of 38080 points, 75446 faces, and 18802 cells.

Upon closer inspection, the created mesh is actually structured, i.e. regular in space. However, the solver created for this work will treat it as unstructured, so no fixed pattern in the cells connectivity is assumed.

\subsubsection{Sub-mesh selection}

A substantial and critical part of the novel adaptive POD method presented is the fact that, during the ROM integration, only a reduced amount of the cells (sub-mesh), in which the computational fluid domain is discretized, is actually evolved in time, and the remaining cells just act, in some sense, as "slave" to the "master" cells through the POD modes. Thus, the master cells need to be distributed in the computational domain in such a way that they allow the enforcement of the boundary conditions and transmission of information between the different regions. They should contain the interesting dynamics of the problem as well. Regions with known numerical noise, like the trailing edge of lifting surfaces, should be avoided.

Several methods have been proposed in literature, such as missing point estimation (Astrid et al., 2008) and hyper-reduction (Ryckelynck, 2009). These methods somehow optimize the sub-mesh selection according to the expected dynamics and/or the nature of the operators acting on the POD modes.

However, since the goal is to adapt to unknown possible dynamics, a more robust (and simpler) procedure is used here. A series of (radial) lines are traced at regular intervals from the airfoil wall to far-field faces, and 960 cells (see figure 5.4) in total are selected along these lines. No additional points are included to capture the shock that is present in the high transonic flow considered below. This is because the position of the shock is unknown, and the sub-mesh is selected beforehand. Of course, an adaptive selection would be more convenient, but this is well ahead the scope of the work presented in this thesis. 


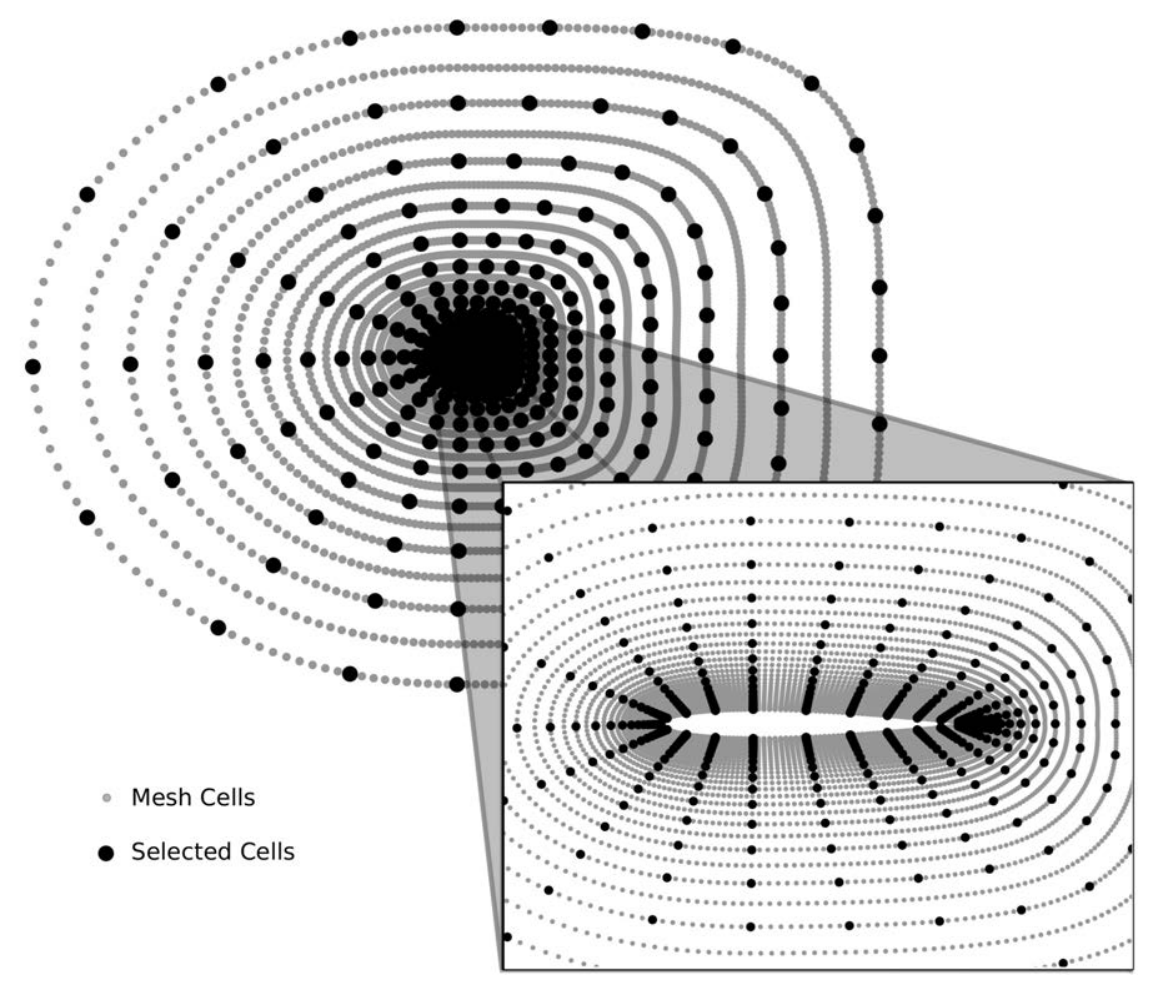

Figure 5.4: Sub-mesh selection

This sub-mesh is used both to calculate the POD modes and to project the governing equations. Note, invoking equation (2.30), that the inner product based on these $M_{0}=960$ cells can be written as defined in equation (2.29). The least squares minimization of the governing equations residuals based on these $M_{0}$ mesh points (see §2.2.3) is performed similarly.

\subsubsection{Oscillatory forcing}

The unsteady flow field is forced by monochromatic pitch or heave oscillations of the airfoil, with either the angle of attack or the vertical position of the airfoil varying as $A o A=a[\cos (2 \pi f t)-1]$ or $h=a \cos (2 \pi f t$ ) (see figure 5.5). For convenience, the reduced frequency (which is nondimensional and proportional to the Strouhal number), is defined in terms of the frequency $f$, the incoming velocity $u_{\infty}$, and the chord $c$, as

$$
k=\pi f c / u_{\infty}
$$

As anticipated, we consider pitch and heave motions with the following combinations of the forcing amplitude

$$
\text { pitch: } a=0.01 \text { and } 0.1 \mathrm{rad}, \text { heave: } a=0.05 c \text {, }
$$



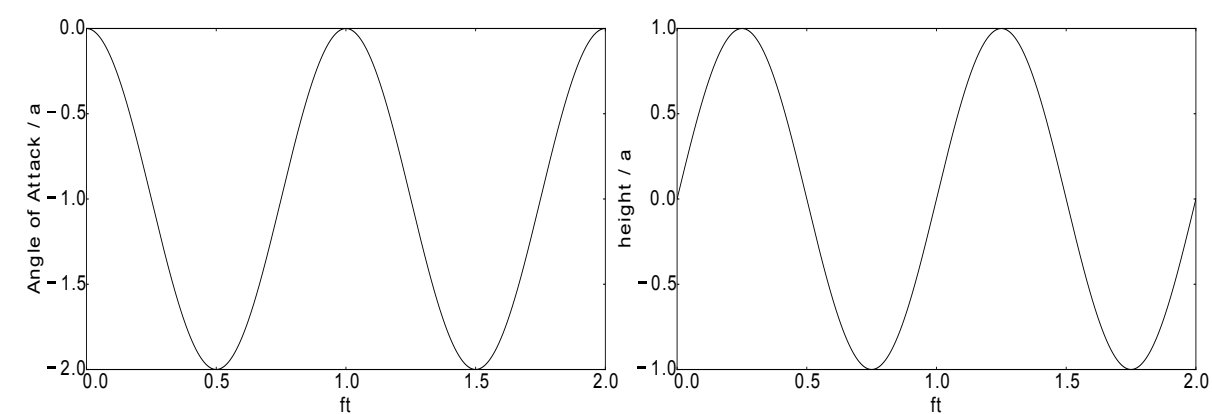

Figure 5.5: Rescaled forcing function for the pitch (left) and heave (right) motions, plotting the rescaled angle of attack and height vs. $f \cdot t$

and two forcing frequencies, one giving a small value of the reduced frequency $k$ and another giving $k \sim 0.5$. Thus, we have six combinations for the forcing mode, which moreover will be considered in the next two subsections for subsonic $(M=0.2)$ and transonic $(M=0.8)$ cases.

\subsubsection{Results for the time dependent subsonic flow at $M=0.2$}

As a first case to test the adaptive POD on the fly methodology, we consider the low subsonic regime, $\mathrm{M}=0.2$, at sea level altitude.

In an initial step, a steady simulation has been carried out to achieve the converged steady state. The unsteady simulation is started subsequently using the full numerical solver for a time $T_{0}^{\mathrm{CFD}}=0.1$, point at which the ROM takes over. For this case, to ensure a seamless transition between the full numerical solver and the ROM the reference value $\mathcal{Q}_{0}$ in equation (2.16) has been taken as the last snapshot corresponding to the full numerical solver, ensuring that $\xi$ equals 0 for the initial ROM time step. Additionally, the POD modes are calculated with respect to the initial steady state.

The ROM residuals are minimized in a least square sense (see equation (2.18)) making use of a Gauss-Newton algorithm (Wright and Nocedal, 1999, pp. 67-68), such that if $\mathfrak{J}$ is the Jacobian of the ROM residual $\mathfrak{R}$ with respect to the POD modes coordinates $\xi$, defined as

$$
\begin{gathered}
\mathfrak{R}_{i}=\sum_{j=1}^{N} \Phi_{i j}\left(\frac{\mathcal{V}_{i}^{n+1} \xi_{j}^{n+1}-\mathcal{V}_{i}^{n} \xi_{j}^{n}}{\Delta t}\right)+\sum_{k} \frac{\partial \mathcal{R}_{i}^{n}}{\partial \mathcal{Q}^{k}} \sum_{j=1}^{N} \Phi_{k j}\left(\xi_{j}^{n+1}-\xi_{j}^{n}\right)+\mathcal{R}_{i}^{n}=0 \\
\mathfrak{J}_{i j}=\Phi_{i j} \frac{\mathcal{V}^{i, n+1}}{\Delta t}+\sum_{k} \frac{\partial \mathcal{R}^{i, n}}{\partial \mathcal{Q}^{k}} \Phi_{k j}
\end{gathered}
$$

the incremental step to the values of $\xi$ that would reduce the residual norm is given as

$$
\Delta \xi^{n+1}=-\left(\mathfrak{J}^{T} \mathfrak{J}\right)^{-1}\left(\mathfrak{J}^{T} \mathfrak{R}\right)
$$

The Gauss-Newton algorithm is applied until convergence in the forces applied over 
the body and in the norm of the residuals has been achieved, or until a maximum number of iterations has been reached.

The tunable parameters of the problem, appearing in $\S 2.3 .2$, are selected for the subsonic case considered here as

$$
T_{0}^{\mathrm{CFD}}=0.1 \mathrm{~s}, \quad \Delta T^{\mathrm{CFD}}=0.1 \mathrm{~s}, \quad \epsilon_{1}=10^{-8}, \quad K=100
$$

In order to emphasize the robustness of the method, these tunable parameters will be maintained in all cases considered below in the subsonic case. Moreover, the CFD update run duration, $\Delta T^{\mathrm{CFD}}$, will be maintained along the simulation. The performance of the POD on the fly method would be increased by varying this parameter along the simulation, noting that as more updates are performed, the quality of the POD modes increases and thus the subsequent CFD update intervals could be decreased. Such selection of $\Delta T^{\text {CFD }}$ could be dynamically chosen along the simulation in an adaptive way (Rapún et al., 2015), but this improvement is beyond the scope of this thesis.

For the pitch oscillations at the lowest forcing amplitude in equation (5.3) and a small forcing frequency, $f=2.5 \mathrm{~Hz}$, which in the present case gives a reduced frequency of $k=0.12$, the outcome of the POD on the fly method is given in figure 5.6. The curves labeled NS are obtained using the CFD solver alone, while those labeled ROM are obtained combining the CFD solver and the low-dimensional ROM, using the POD on the fly method. As it can be seen, the length of the time intervals during which the ROM is used (shaded region in figure 5.6) steadily increases along the simulation,
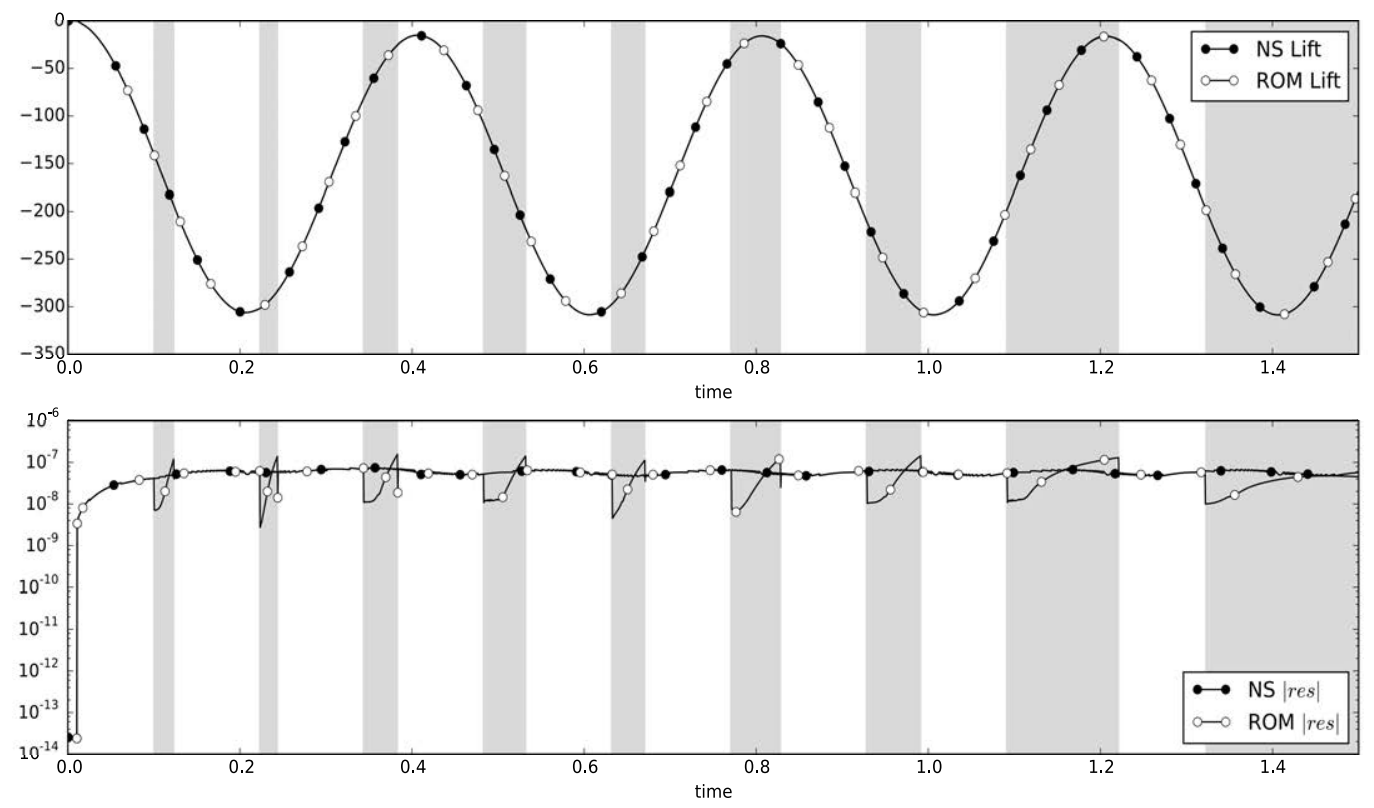

Figure 5.6: POD on the fly vs. the CFD solver, with $\mathrm{M}=0.2$ at sea level, considering pitch motion with $a=0.01 \mathrm{rad}$ and $f=2.5 \mathrm{~Hz}$. Top: the nondimensional lift vs. time, with those intervals in which the low dimensional ROM is used in the POD on the fly method indicated with shaded regions. Bottom: the residuals vs. time 
meaning that the updating strategy described in $\S 2.3 .2$ is very efficient to improve the POD modes as more information from the CFD solver (namely, a larger number of snapshots) is accounted for.

The CPU acceleration factor, defined as

$$
\text { CPU acceleration }=\frac{\text { NS CPU time }}{\text { ROM CPU time }}
$$

equals 1.6 in the considered time interval $(0 \leq t \leq 1.5 \mathrm{~s})$ and is still larger in larger time intervals (because the length of the intervals in which the ROM is used steadily increases). The upper plot also shows that the lift provided by both, the CFD alone and the POD on the fly methods, are plot-indistinguishable. Plot indistinguishable approximations mean hereinafter that the associated relative errors are smaller than $1 \%$. Moreover, the ROM lift curve does not show any artifacts at those values of $t$ in which the POD on the fly method switches between the CFD solver and the ROM. All these are consistent with the very small and comparable residuals of both the CFD solver and the POD on the fly ROM (lower plot). In fact, the plots of the residuals vs. time will be qualitatively similar in the remaining results below and, consequently, the counterpart of the lower plot will be omitted.

In the subsonic case, the performance of the ROM slightly worsens as either the pitch amplitude or frequency are increased. However, even for the largest selected amplitude in equation (5.3) and the largest considered frequency, the performance of the POD on the fly method is still reasonable. This is seen in figure 5.7, which shows that the total length of the time intervals in which the low dimensional ROM is used in the POD on the fly method is smaller than in figure 5.6. Consequently, the CPU acceleration factor defined in equation (5.7) is also smaller, namely CPU acceleration $\sim 1$.3. The approximation of the lift is as good as in the former case and, moreover, the pressure field is also very well approximated, which is seen in figure 5.8. Note that both pressure fields are plot indistinguishable.

The results above are concerned with pitch motions. Several tests for heave motions (omitted here) have shown that these motions are less demanding than pitch motions for the POD on the fly performance. This is due to the fact that the airfoil heave motion produces a constant incremental downwash along the airfoil chord.

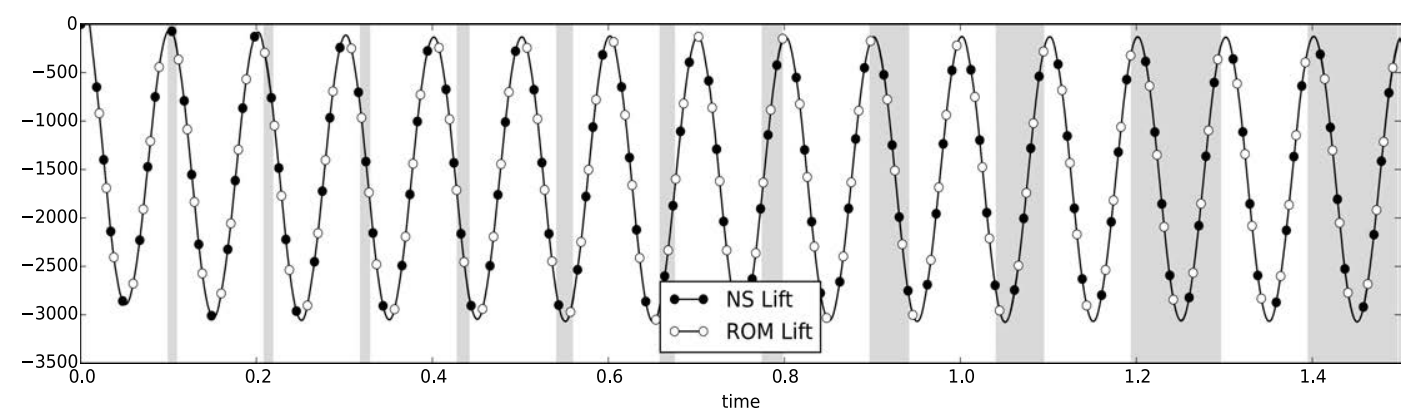

Figure 5.7: Counterpart of figure 5.6-top for $a=0.1 \mathrm{rad}$ and $f=10 \mathrm{~Hz}(k=0.48)$. 

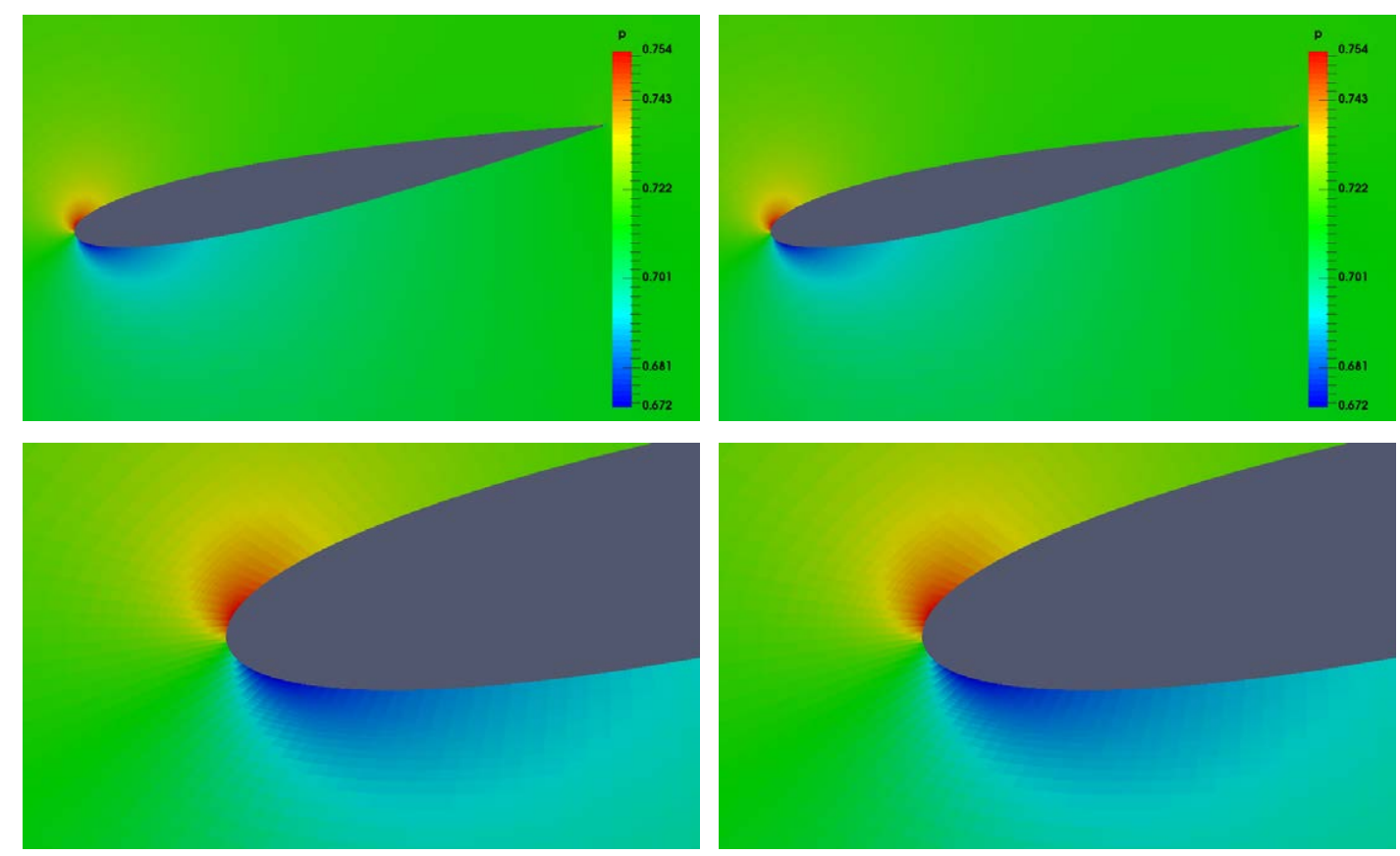

Figure 5.8: Complete color maps (top) and blow up near the leading edge (bottom) of the instantaneous (rescaled) pressure distributions of the run in figure 5.7 at the minimum angle of attack ( $f t=1 / 2$, see figure 6.7), as obtained using the CFD alone (left) and the POD on the fly method (right)

In summary, the POD on the fly method simulates the flow in a robust and efficient way. In particular, the truncation instability is avoided and the updating strategy recognizes well the relevant dynamical information. As expected, the performance of the POD on the fly method slightly worsens as either the forcing amplitude or frequency increases. However, the results are reasonably good even for the worst considered case.

\subsubsection{Results for the time dependent transonic flow at $M=0.8$}

Let us now increase the Mach number to $\mathrm{M}=0.8$, maintaining the sea level altitude to facilitate comparison with the subsonic case. For this transonic flow, the NACA0010 airfoil exhibits strong shocks in the pressure and suction sides of the airfoil, even at zero angle of attack. The presence of such discontinuities makes the ROM operation more challenging, since it needs to predict the quite noticeable shock movement as the angle of attack varies. Moving discontinuities are specially demanding for POD because, in the end, the POD modes are linear combinations of the snapshots, and the linear combination of shifted discontinuities is not a discontinuity, but a stair-like structure. This difficulty was already illustrated for steady transonic aerodynamics (Alonso et al., 2010), where a specific (fairly involved and difficult to implement in three space dimensions) shock treatment was developed. Such treatment is not used here, meaning that the updating strategy must adapt the POD modes to capture well the discontinuities.

The solution has been performed similarly as in the subsonic case presented above, 
with the exception that for the present transonic case, due to the presence of moving shocks, it is convenient that the updating events be more frequent. In other words, it is necessary that the updated POD modes correspond to not too different positions of the shocks. More frequent updating events can be forced by selecting smaller duration of the CFD update intervals in which the new snapshots are calculated. Thus, a lower value of $\Delta T^{\mathrm{CFD}}$ than for the subsonic case has been proven to show better ROM efficiency. Specifically, the tunable parameters of the POD on the fly method appearing in $\S 2.3 .2$ are now selected as

$$
T_{0}^{\mathrm{CFD}}=0.1 \mathrm{~s}, \quad \Delta T^{\mathrm{CFD}}=0.005 \mathrm{~s}, \quad \epsilon_{1}=10^{-8}, \quad K=100
$$

Comparison with the tunable parameters selected in the subsonic case, in equation (5.1.4), shows that all tunable parameters coincide except for $\Delta T^{\mathrm{CFD}}$, which further emphasizes the robustness of the method.

In principle, since the ROM needs to capture the shock motion, one could expect that the performance of the POD on the fly method worsens as either the forcing amplitude or frequency increases. Anticipating the results below, this conclusion turns out to be true for the forcing amplitude, but not in connection with the frequency. This is because large forcing frequencies do not necessarily promote a larger back and forth motion of the shock wave, but a smaller motion. In other words, for a given forcing amplitude $a$, the amplitude of the motion of the shock wave decreases as $f$ increases. This is due to the fact that for a large forcing frequency the shock is not able to move as fast as the airfoil moves.

As in the former subsection, we first concentrate in pitch motions. For the transonic case, the maximum pitch amplitude at the maximum frequency is a too extreme case for which the CFD run does not converge. This is seemingly due to the fact that the considered mesh and time intervals are not fine enough for this case. Hence, the effects of the forcing amplitude and frequency are shown individually in figure 5.9. As it can be seen, the performance of the ROM is qualitatively similar (in fact, even better in terms of the CPU acceleration factor) to that for the subsonic case considered in the former subsection. Note in particular that, as expected, the acceleration factor decreases as either the forcing frequency or the forcing amplitude increase, and it is more sensitive to increasing the forcing amplitude, as anticipated.

The oscillations of the residual (not shown in the figure) are somewhat larger and the timespans in which the ROM approximation is acceptable are smaller (and evolve somewhat irregularly) than in the subsonic case. However, the POD on the fly ROM still works fairly well. In particular, the relevant POD modes are efficiently captured as time increases. It is also obvious in the figure that the length of the intervals in which the low-dimensional ROM is used is fairly small after the first computation of the POD modes. This is because the POD modes do not contain initially enough information on the shock movement. However, the method recovers well and the lengths of the ROM intervals increase. All these are in spite of the fact that, in this unsteady simulation, 

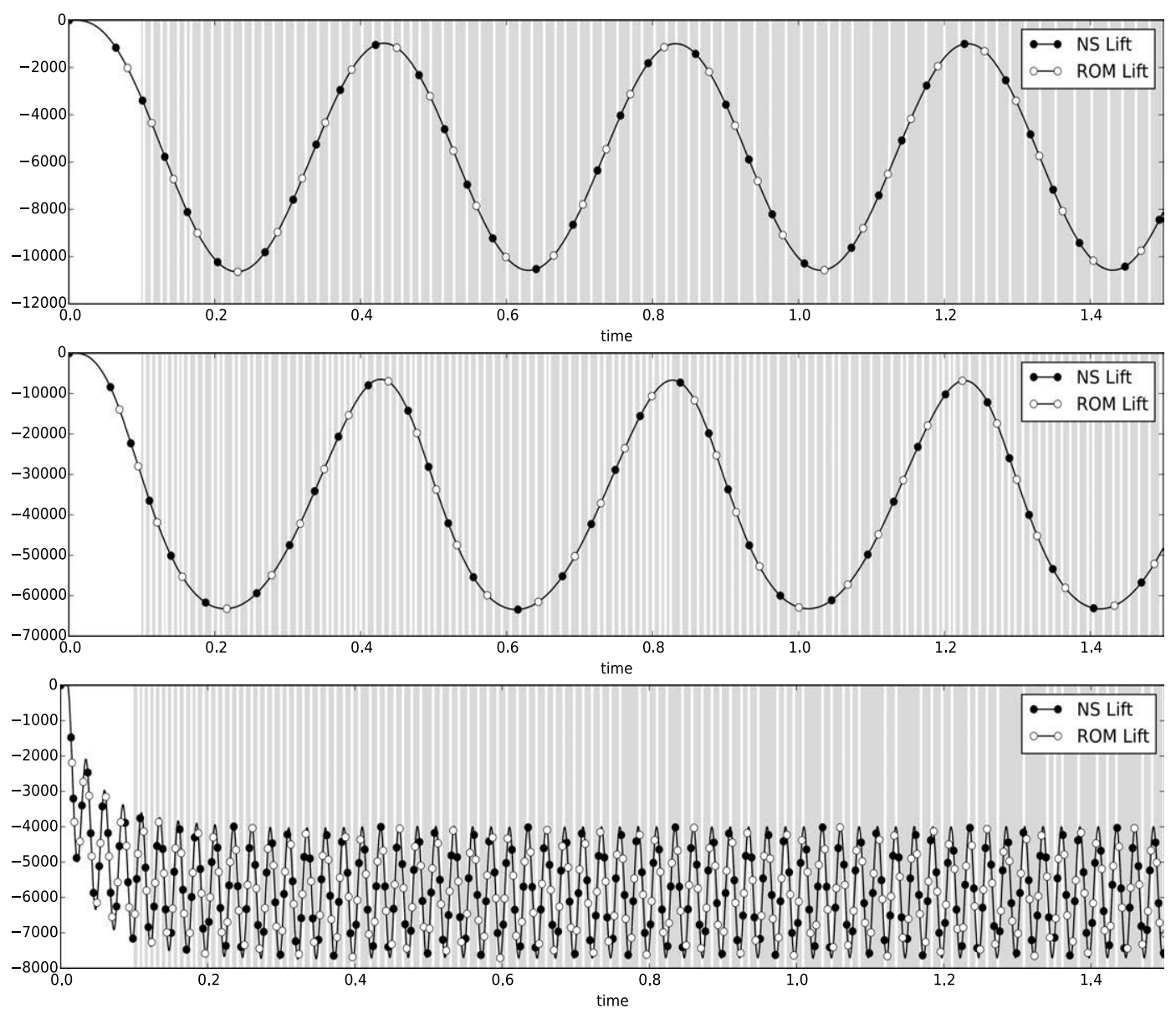

Figure 5.9: Counterpart of figure 5.6-top for $\mathrm{M}=0.8$, considering the following values of the forcing amplitude and frequency: $a=0.01 \mathrm{rad}, f=2.5 \mathrm{~Hz}, k=0.029$ (top); $a=0.1 \mathrm{rad}, f=2.5 \mathrm{~Hz}, k=0.029$ (middle) and $a=0.1 \mathrm{rad}, f=40 \mathrm{~Hz}, k=0.46$ (bottom). The CPU acceleration factors defined in equation (5.7) are 2.3, 1.7, and 2.2 in the upper, middle and lower plots, respectively.

the shocks significantly move along the pressure and suction sides of the airfoil, as seen in figure 5.10. Note that the pressure fields calculated with the ROM and the standard CFD solver are plot indistinguishable. In these plots, consistently with the CFD computed value using a cell centered finite volume scheme, a constant value of the pressure field is represented on each cell. Thus, the (apparent) artifacts appearing in the plots are solely due to the fact that the mesh is fairly coarse except in the vicinity of the airfoil.

As in the subsonic case, the heave motion has been shown to be a less demanding condition for the ROM efficiency and robustness. 

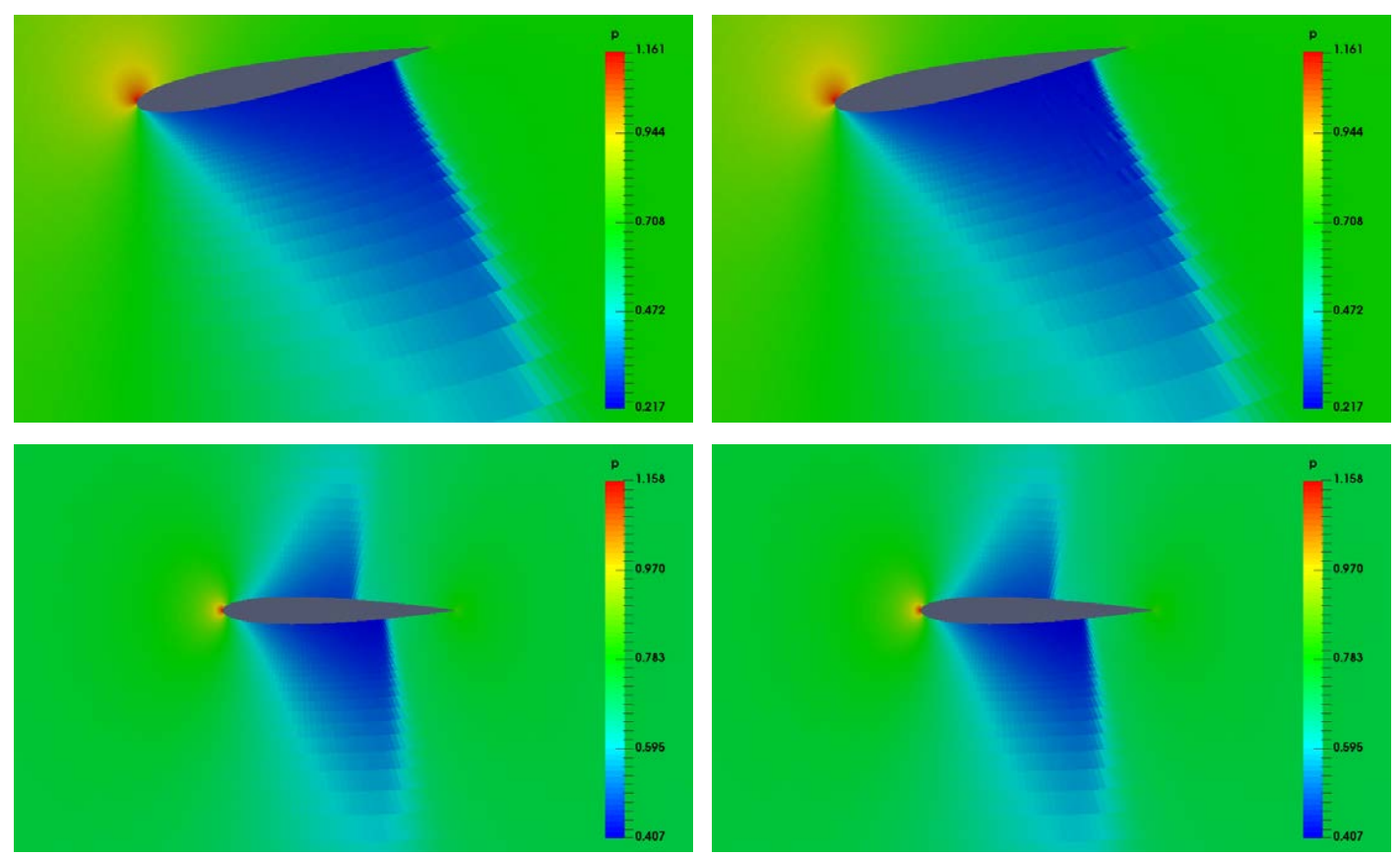

Figure 5.10: Counterpart of figure 5.8 for the run considered in figure 5.9-middle plot at times with a minimum (top) and zero (bottom) angle of attack (namely, at $f t=1 / 2$ and $f t=1$, respectively, see figure 5.5)

\subsection{Remarks on solution methods and ROM efficiency}

\subsubsection{Number of modes}

Now, if the computational cost of the ROM were negligible compared to that of the CFD solver, the CPU acceleration factor defined in equation (5.7) would be proportional to the total timespan in which the ROM is used, which is calculated by adding up the lengths of the various time intervals in which the low-dimensional ROM is used. However, the computational cost of the ROM is not negligible. Instead, it increases as the number of retained modes increases. Thus, it is convenient to also take into account the required number of POD modes, which are considered in figure 5.11. As shown, the number of modes increases as either the forcing amplitude or frequency increases. Moreover, this increase is larger when increasing the forcing frequency than when increasing the forcing amplitude. However, in the latter case, the timespans in which the ROM is used are larger than in the former case (see figure 5.9), which compensates the larger number of retained POD modes.

In any event, a further increase in the computational efficiency may result by a better selection of the the CFD update run duration, $\Delta T^{\mathrm{CFD}}$, as anticipated, and also by using generic mode libraries (Rapún et al., 2015), which highly reduces the required value of the numerical solver update run duration, increasing the overall efficiency of the method when simulating very complex dynamics in the Complex Ginzburg-Landau equation. 


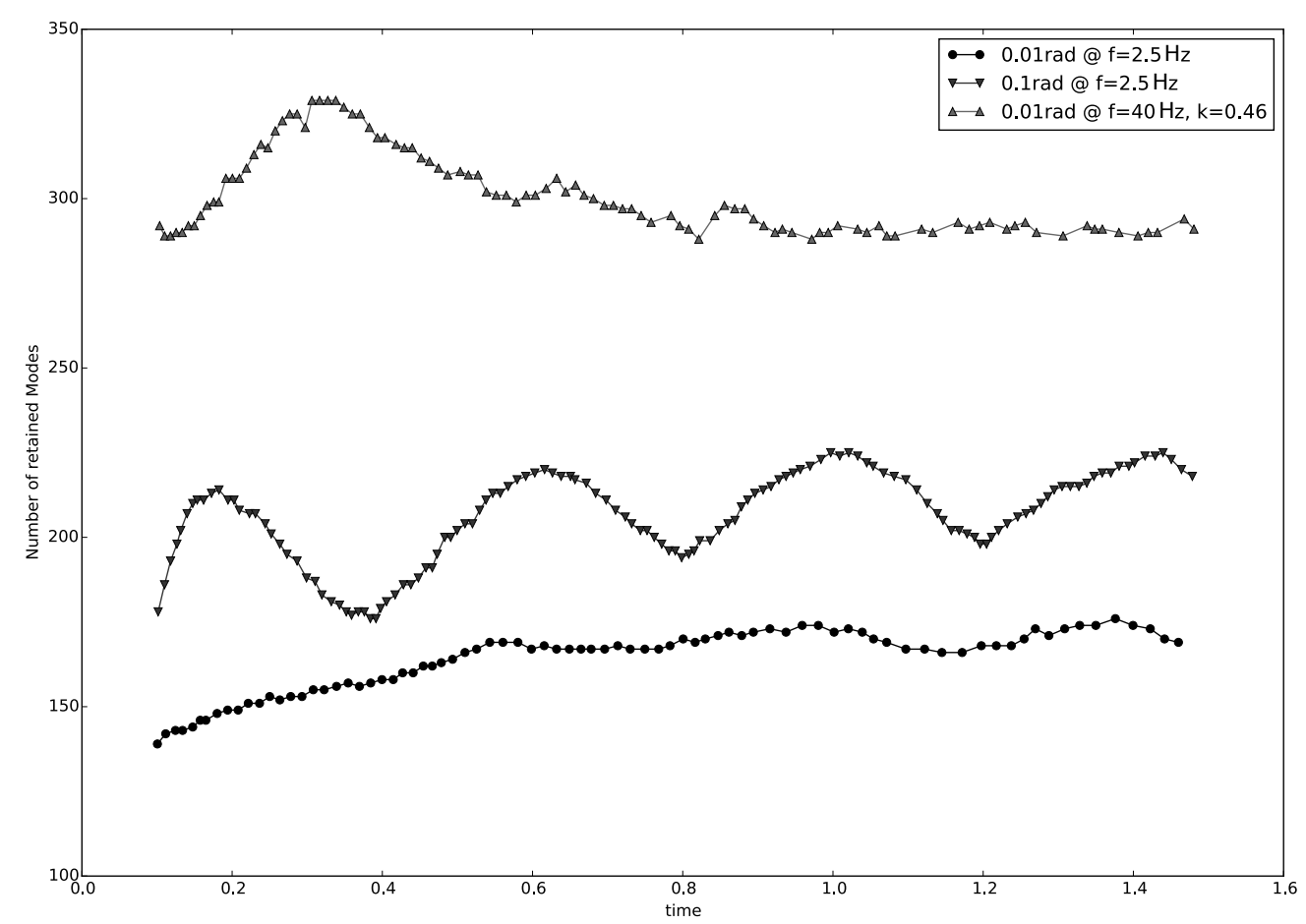

Figure 5.11: POD modes number evolution along the various timesteps performed by the POD on the fly ROM for the $\mathrm{M}=0.8$ case.

\subsubsection{Solution method and tunable parameters effects}

In the previous sections the selection of parameters and solution method used has been highlighted. Let's turn now the attention to the effect of different solution methods and parameters on the ROM efficiency. For this, the efficiency of the ROM for different values and solution strategies will be evaluated in the subsonic case at low amplitude and low reduced frequency.

\section{Effect of $\triangle T^{\mathrm{CFD}}$}

The effect of $\Delta T^{\mathrm{CFD}}$ has been studied by running different cases with values of 0.1 , $0.05,0.01$ and $0.005 \mathrm{~s}$ while keeping the remaining parameters and solution strategies unchanged. From the comparison in figure 5.12, it can be seen that, in general, the smaller the value of $\Delta T^{\mathrm{CFD}}$ the more efficient the ROM is. This has, however, a secondary consequence due to the ROM updating strategy, since the smallest value of $\Delta T^{\mathrm{CFD}}$ does not seem to keep enough modes to be able to advance the solution without having to revert to the NS (at least for the amount of time run) as it is the case for the 0.10 and 0.05 values.

\section{Effect of $\varepsilon_{1}$}

As highlighted in equation (2.21) the value of $\varepsilon_{1}$ controls directly the error of the POD approximation. The effect of reducing its value has been studied by, without modifying 


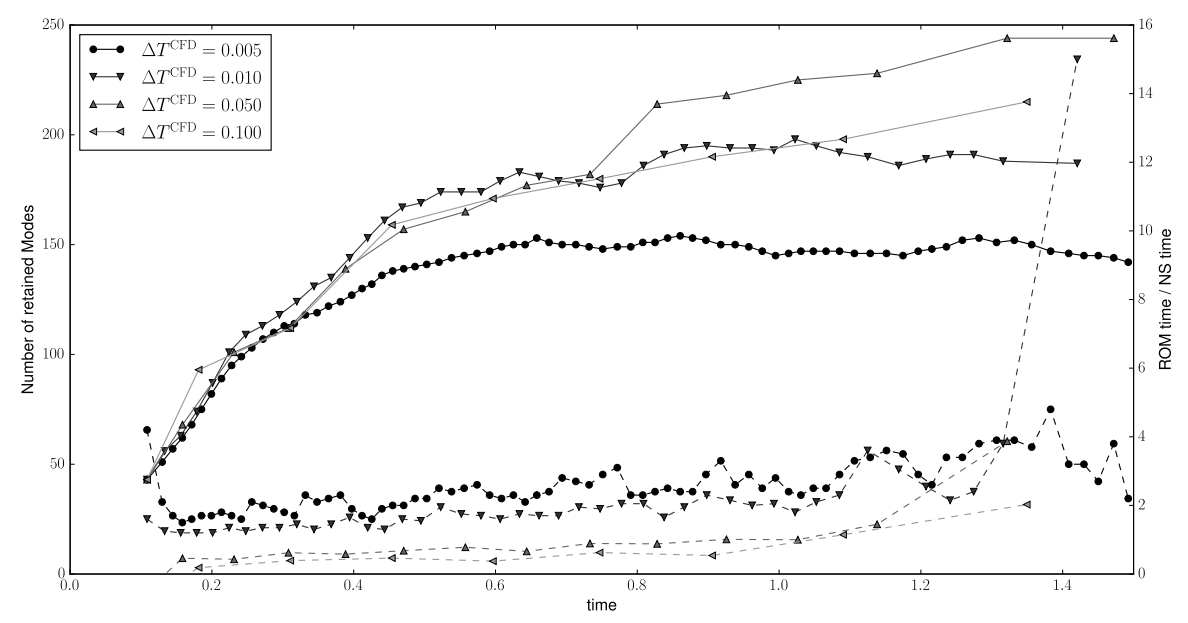

Figure 5.12: $\triangle T^{\mathrm{CFD}}$ effect on the ROM efficiency (--) and number of retained modes (-) for the $\mathrm{M}=0.2$ pitch motion with $a=0.01 \mathrm{rad}$ and $f=2.5 \mathrm{~Hz}$

any parameter or solution strategy, running two simulations for the cases of $\varepsilon_{1}=10^{-8}$ (the nominal) and $\varepsilon_{1}=10^{-12}$. As it can be seen in figure 5.13, the number of modes significantly increases, but the efficiency of the ROM remains equivalent.

If the effect is studied in conjunction with $\Delta T^{\mathrm{CFD}}=0.005$ the effect is slightly different. It can be seen in figure 5.14 how, starting at $t \approx 1 \mathrm{~s}$, the efficiency of the ROM starts to increase and the number of modes stabilizes for the lower $\varepsilon_{1}$ case.

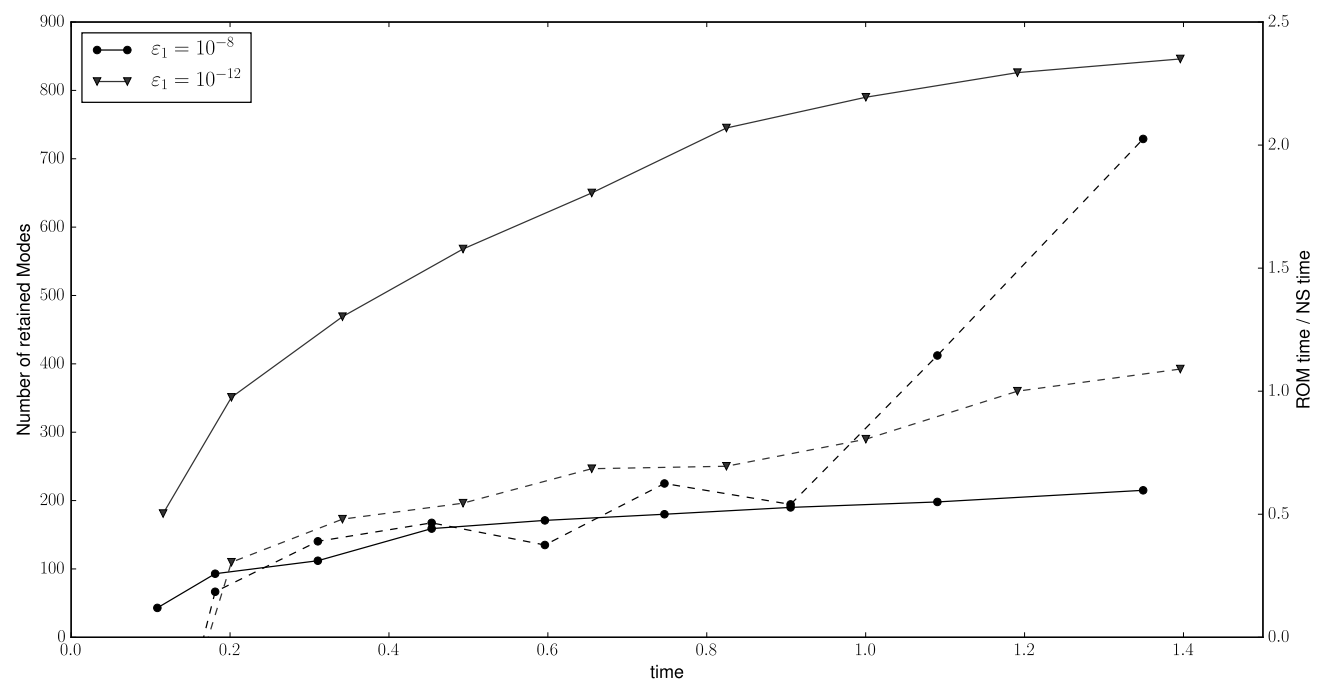

Figure 5.13: $\varepsilon_{1}$ effect on the ROM efficiency (--) and number of retained modes $(-)$ for the $\mathrm{M}=0.2$ pitch motion with $a=0.01 \mathrm{rad}$ and $f=2.5 \mathrm{~Hz} \Delta T^{\mathrm{CFD}}=0.10$

\section{Effect of the selection of $\mathcal{Q}_{0}$}

The baseline runs have been performed using as $\mathcal{Q}_{0}$ the data from the last snapshot, and the POD modes have been calculated using the delta of the snapshots with respect to the steady state. If the POD modes are calculated without using any reference, it 


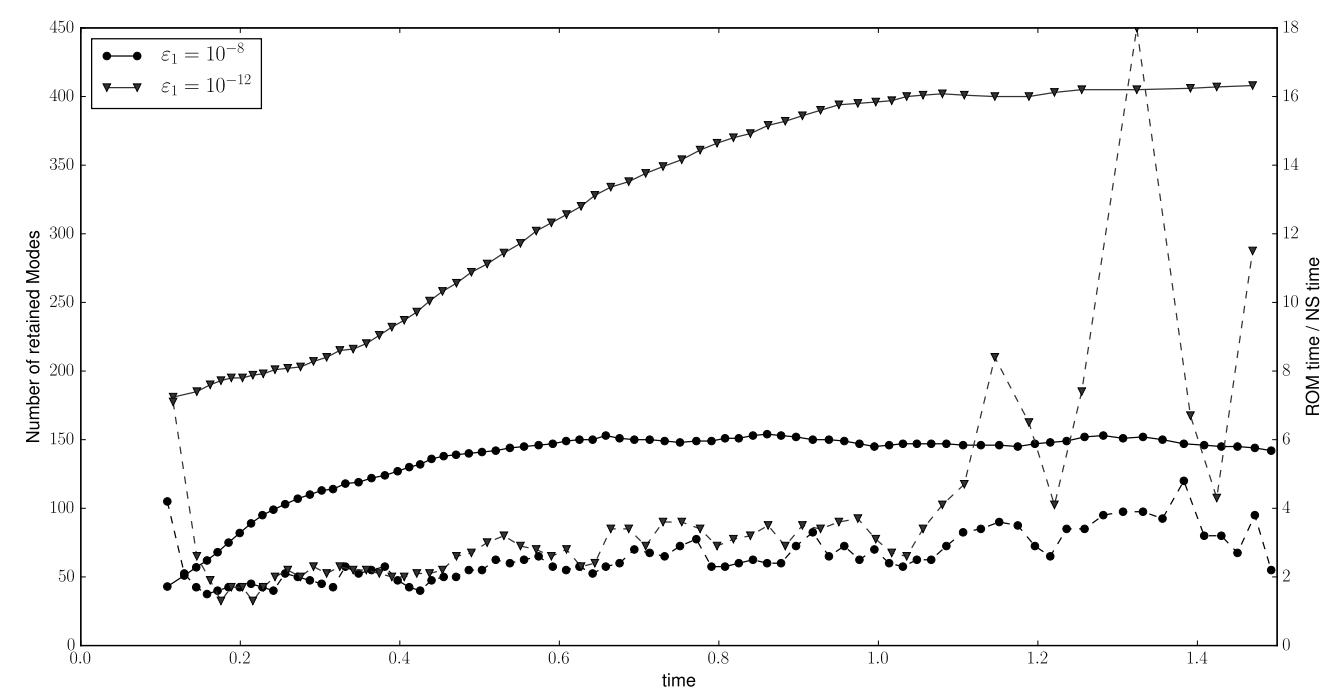

Figure 5.14: $\varepsilon_{1}$ effect on the ROM efficiency (--) and number of retained modes (-) for the $\mathrm{M}=0.2$ pitch motion with $a=0.01 \mathrm{rad}$ and $f=2.5 \mathrm{~Hz} \Delta T^{\mathrm{CFD}}=0.005$

makes sense to set $\mathcal{Q}_{0}=(0,0, \ldots, 0)$. In this case, the initial values of $\xi$ are not null, they are set as

$$
\xi_{0}=\left\langle\Phi^{T}, \mathcal{Q}_{N}\right\rangle
$$

such that the error in the ROM initial step depends on the value of $\varepsilon_{1}$. As it can be seen in figure 5.15, the performance of the ROM projection without reference is significantly worse, since, for the selected value of $\varepsilon_{1}=10^{-8}$, not enough POD modes are available in the basis to be able to reproduce the flow field accurately, much less to evolve the flow in time efficiently. If the value of $\varepsilon_{1}$ is reduced to the extreme value of $10^{-12}$, the availability of enough POD modes in the basis is not an impediment for the efficiency of the ROM and approximately the same ROM efficiency numbers are obtained (see figure 5.16), but the computational cost is higher since the number of retained modes is more than double. A similar trend occurs for the case in which $\Delta T^{\mathrm{CFD}}=0.005$, as can be seen in figure 5.17 .

\section{Least squares residual minimization vs Galerkin projection}

Traditionally, POD based ROMs are evolved in time making use of the Galerkin projection, although for the work presented in this thesis the least squares residual minimization has been found to be a more robust and better performing method. Due to the nature of the Galerkin projection, it has been performed for this check without any reference $\mathcal{Q}_{0}$ for the projection, and it has been solved making use of a fixed point iteration. The comparison between the different methods using the $L_{E}$ and $\ell^{2}$ scalar product definition can be found in figure 5.18. As it can be seen, the Galerkin projection using the $L_{E}$ inner product definition completely fails to evolve the solution in time. If the $\ell^{2}$ is used, the ROM is only capable of evolving the solution for small time intervals. The same conclusion is extracted for the lower value of $\Delta T^{\mathrm{CFD}}=0.005$, as 


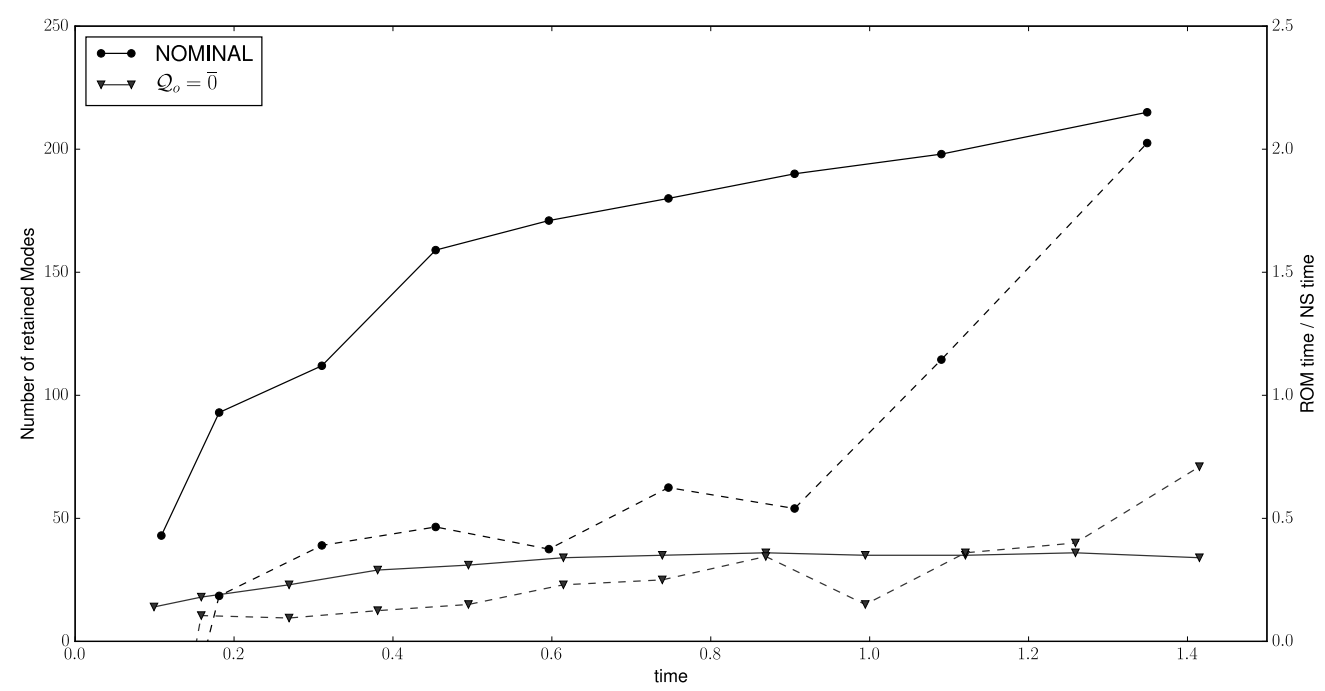

Figure 5.15: $\mathcal{Q}_{0}$ effect on the ROM efficiency (-- ) and number of retained modes $(-)$ for the $\mathrm{M}=0.2$ pitch motion with $a=0.01 \mathrm{rad}$ and $f=2.5 \mathrm{~Hz} . \Delta T^{\mathrm{CFD}}=0.10$, $\varepsilon_{1}=10^{-8}$ effect

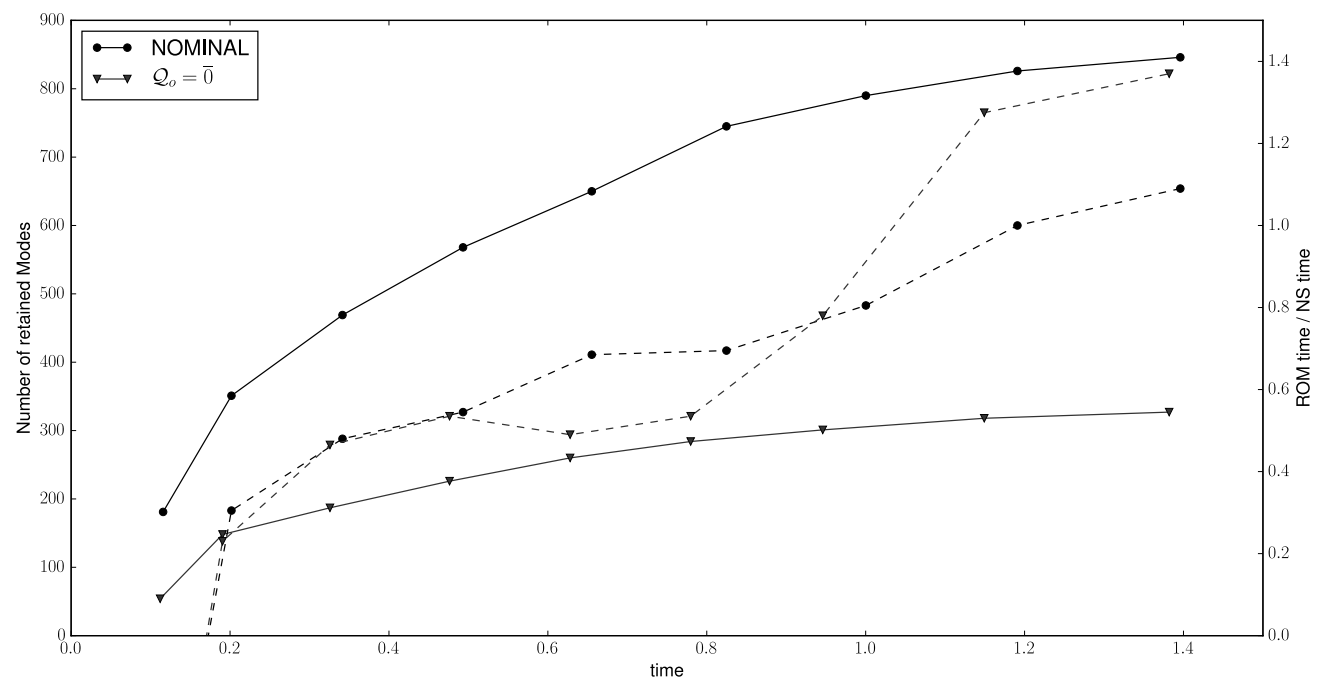

Figure 5.16: $\mathcal{Q}_{0}$ effect on the ROM efficiency (-- ) and number of retained modes $(-)$ for the $\mathrm{M}=0.2$ pitch motion with $a=0.01 \mathrm{rad}$ and $f=2.5 \mathrm{~Hz} . \Delta T^{\mathrm{CFD}}=0.10$, $\varepsilon_{1}=10^{-12}$ effect 


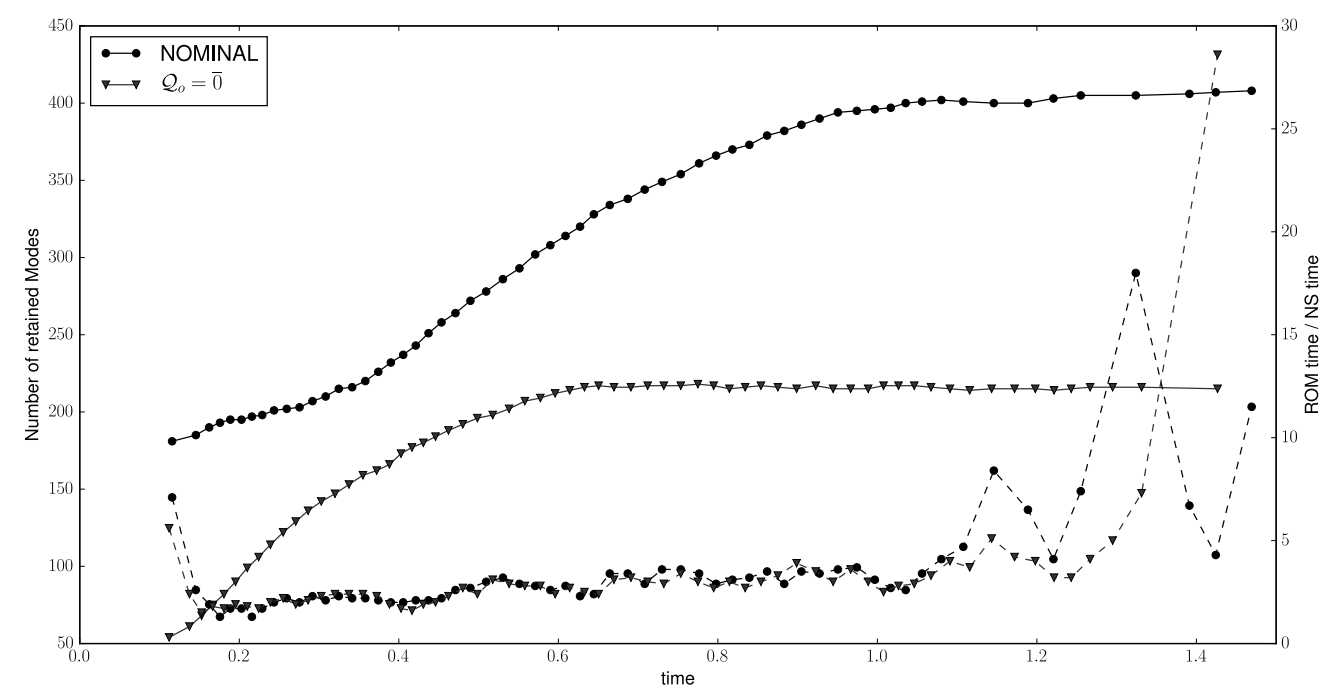

Figure 5.17: $\mathcal{Q}_{0}$ effect on the ROM efficiency (-- ) and number of retained modes $(-)$ for the $\mathrm{M}=0.2$ pitch motion with $a=0.01 \mathrm{rad}$ and $f=2.5 \mathrm{~Hz} . \Delta T^{\mathrm{CFD}}=0.005$, $\varepsilon_{1}=10^{-12}$ effect

can be seen in figure 5.19 .

There are two scenarios in which the Galerkin projection, minimized with a fixed point iteration, has been seen to fail. In the first one, the projected residual ratio, defined similarly as equation (2.21) as

$$
E_{\text {Proj }_{\text {res }}}=\frac{\left\|\left\langle\Phi, \sum_{j=1}^{N} \Phi_{i j}\left(\frac{\mathcal{\nu}_{i}^{n+1} \xi_{j}^{n+1}-\mathcal{V}_{i}^{n} \xi_{j}^{n}}{\Delta t}\right)+\mathcal{R}_{i}\left(\mathcal{Q}_{o}+\Phi \xi^{n+1}\right)\right\rangle\right\|}{\|\left\langle\Phi,\left(\mathcal{R}_{i}\left(\mathcal{Q}_{o}+\Phi \xi^{n}\right)\right\rangle \|\right.},
$$

is reduced with each sub-iteration, even to a very small number, but the non-projected residual ratio $\left(E_{\text {res }}\right)$ does not get below the threshold $1 / K$, so the simulation is reverted to the full numerical solver. This can be seen, for example, in figure 5.20.

In the second one, the minimization diverges after a small number of sub-iterations. 


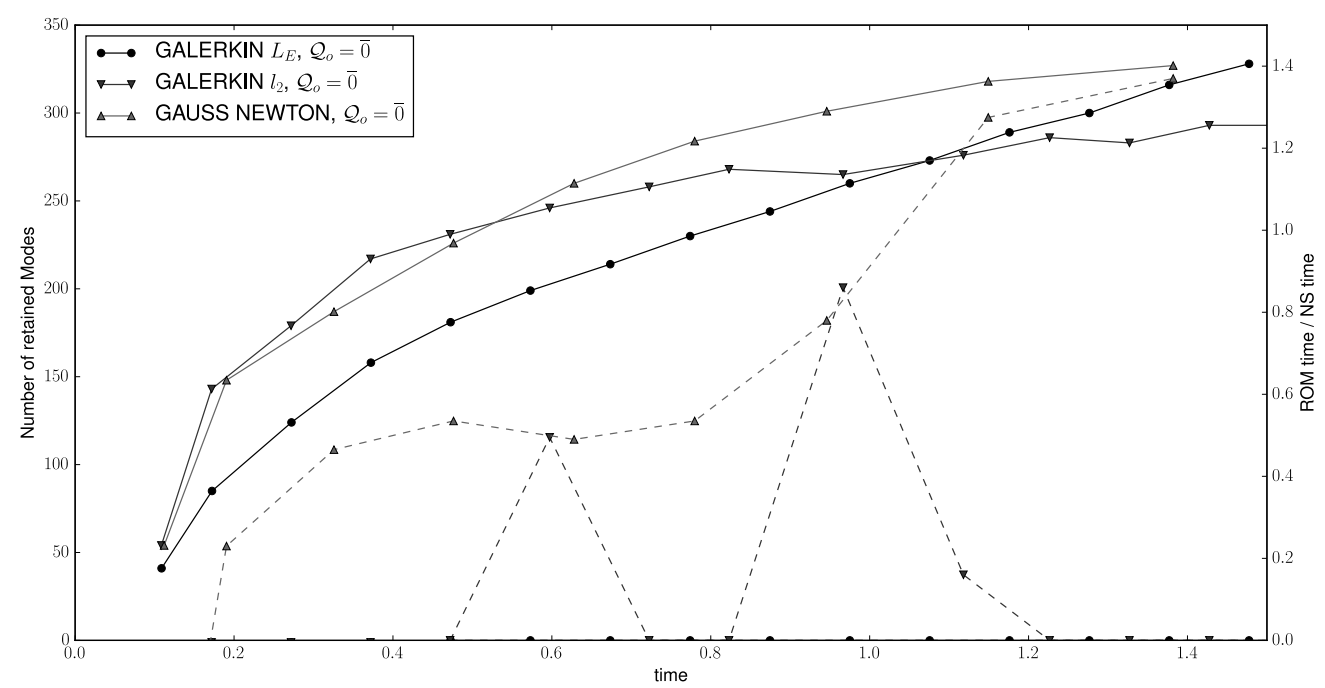

Figure 5.18: Comparison between Gauss Newton residual minimization and Galerkin projection for the $L_{E}$ and $\ell^{2}$ inner product selection effect on the ROM efficiency (--) and number of retained modes $(-)$ for the $\mathrm{M}=0.2$ pitch motion with $a=0.01 \mathrm{rad}$ and $f=2.5 \mathrm{~Hz} . \Delta T^{\mathrm{CFD}}=0.10, \varepsilon_{1}=10^{-12}$ effect

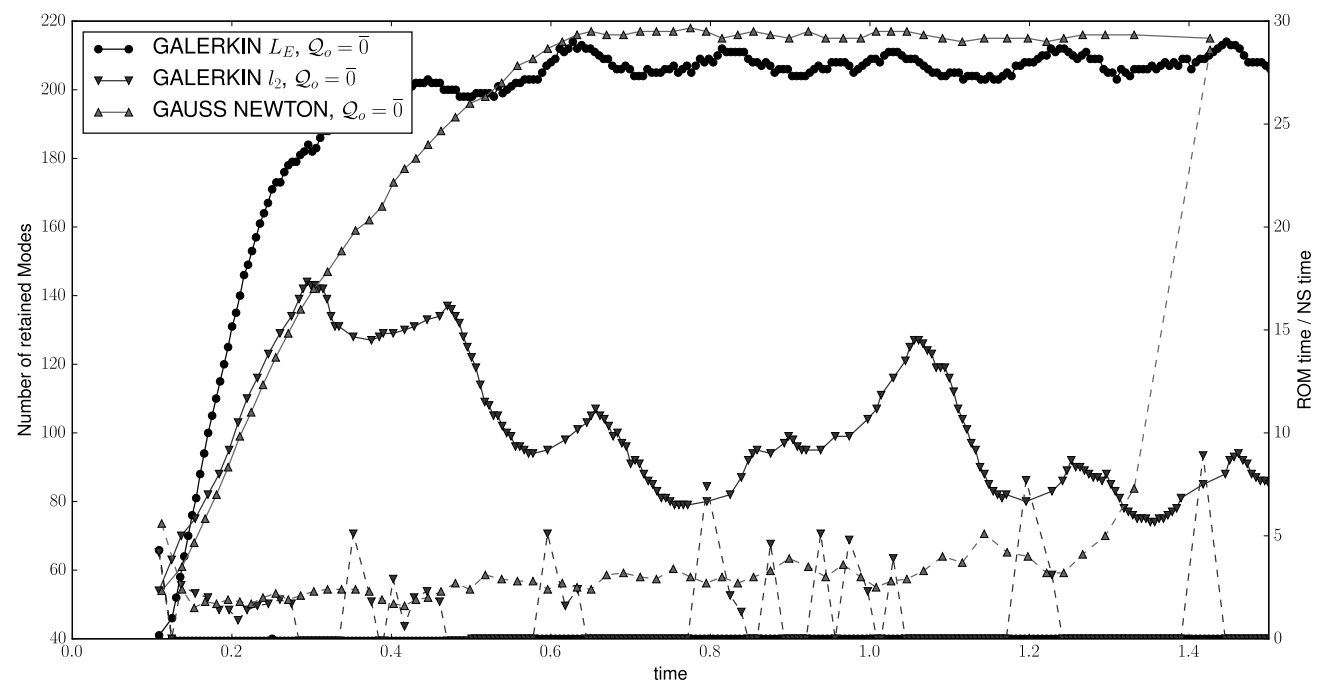

Figure 5.19: Comparison between Gauss Newton residual minimization and Galerkin projection for the $L_{E}$ and $\ell^{2}$ inner product selection effect on the ROM efficiency (-- -) and number of retained modes $(-)$ for the $\mathrm{M}=0.2$ pitch motion with $a=0.01 \mathrm{rad}$ and $f=2.5 \mathrm{~Hz} . \Delta T^{\mathrm{CFD}}=0.005, \varepsilon_{1}=10^{-12}$ effect 


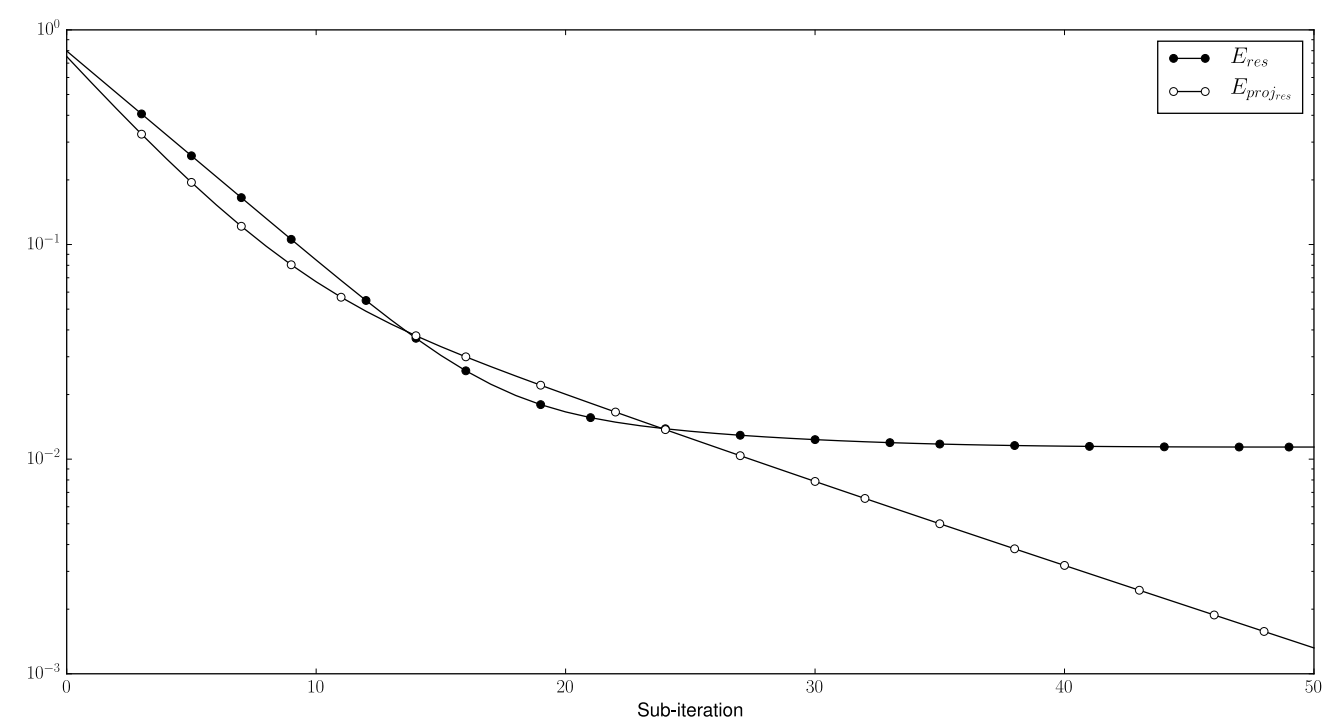

Figure 5.20: $E_{\text {res }}$ vs. $E_{\text {projres }}$ for a Galerkin based ROM residual minimization based on $L_{E}$ inner product

\subsection{Aeroelastic simulation}

Taking the same fluid solver as in the forced oscillation case and the structural model of the airfoil in $\S 4.1 .2$, both are coupled together, such that the fluid forces acting on the interface are able to deform the airfoil. Thus, the numerical mesh needs to deform to conform to the airfoil aerodynamic shape. In this way, aeroelastic simulations can be performed. As above, the fluid solver is composed of a numerical solver and an adaptive ROM, which takes over after a fixed amount of time and reverts back the solution to the full solver automatically when the convergence criteria is no longer fulfilled.

\subsubsection{Structural model}

The structural parameters for the airfoil with control surface (see §4.1.2), have been selected as

$$
\begin{array}{cccc}
x_{\text {rot }}=0.25 \mathrm{~m} & x_{c}=0.5 \mathrm{~m} & x_{\alpha}=0.125 \mathrm{~m} & x_{c s}=0.1 \mathrm{~m} \\
\frac{\omega_{h}}{2 \pi}=6 \mathrm{~Hz} & \frac{\omega_{\theta}}{2 \pi}=12 \mathrm{~Hz} & \frac{\omega_{\beta}}{2 \pi}=7 \mathrm{~Hz} \\
M=100 \mathrm{~kg} \mathrm{~m}^{-1} & \frac{M_{c s}}{M}=0.2 & \frac{I_{\theta}}{M}=0.2 \mathrm{~m}^{2} & \frac{I_{\beta}}{M}=0.003 \mathrm{~m}^{2} \\
g_{h}=g_{\theta}=g_{\beta}=0.005 &
\end{array}
$$

Since these parameters alone do not give a real indication on the qualitative dynamic stability of the system, aeroelasticians typically prefer to look at the eigenvalues and eigenvectors of the system's free vibration in the absence of damping, usually referred to as normal modes. Recalling equation (4.22) and assuming a solution of the form 
$\tilde{x}=\mathcal{U} e^{\lambda t}$, we have the following eigenvalue problem

$$
\lambda \mathcal{U}=-\mathcal{M}^{-1} \mathcal{L} \mathcal{U}
$$

where $\mathcal{U}$ are the eigenvectors of the system and $\lambda$ are the corresponding eigenvalues.

For this particular example, the graphical representation of the eigenvectors and the corresponding eigenvalues imaginary part (recall that since there is no damping the eigenvalues are pure imaginary) can be seen in figure 5.21. Examining closely the normal modes, the first two modes do not involve any airfoil pitch but do include heave and control surface rotations, while the third mode involves no heave but pitching, as well as control surface rotation. From a qualitative perspective, for a trained eye, it is clear that the first two modes are likely to couple together in a binary flutter mode and that, at higher speeds, the third mode might couple with the first and second modes.

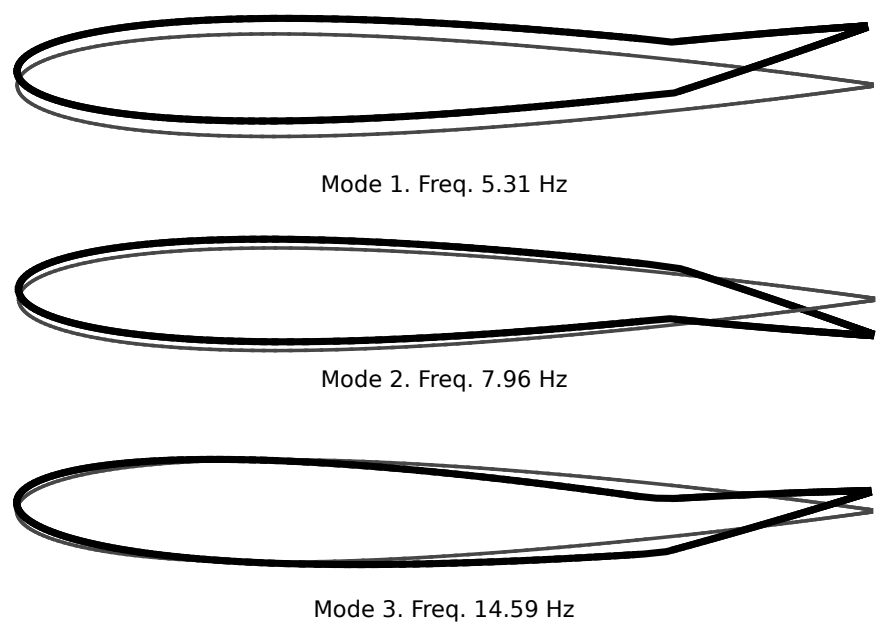

Figure 5.21: Airfoil normal modes

\subsubsection{Analytical flutter solution}

As mentioned above, if the airfoil is assumed to follow a pure harmonic motion of small amplitude, the Theodorsen function can be integrated to obtain an analytical expression for the forces acting on an airfoil as a function of the reduced frequency

$$
k=\frac{\omega c}{2 u_{\infty}} .
$$

If we recall equation (4.22), in the absence of external forces other than the self induced aerodynamic forces, and expressing $\tilde{x}=\mathcal{X} e^{p t}$, where $p$ is the Laplace variable that can be expressed as $p=\gamma \omega+i \omega$ (with $g=2 \gamma$ ), we have finally that

$$
\left[\mathcal{M} p+\mathcal{L}-Q\left(\text { Mach }, q_{\infty}, k\right)\right] \mathcal{X}=0
$$

Since $Q$, which accounts for the aerodynamic forces, depends on the imaginary 
part of $p$, this eigenvalue problem needs to be solved iteratively for a specific Mach number and a set of dynamic pressures $\left(q_{\infty}\right)$. In a first step, the iteration is started for a null dynamic pressure, so the first set of eigenvectors correspond to the structure natural modes, as obtained in equation (5.10). In a second step, the dynamic pressure is increased and the aerodynamic forces updated starting with the imaginary part of the eigenvalues from the first step. For each of the eigenvalues/eigenvector pairs, the new imaginary part of the eigenvalue is obtained, the aerodynamic forces updated and the process is repeated until convergence is achieved. Special care has to be put in following the same eigenvalue/eigenvector pair in each iteration. Once a solution has been obtained for all eigenvalues, the dynamic pressure is further increased and the iterative process starts again. This procedure is known as the p-k method (Hassig, 1971).

Finally, the complete evolution of the eigenvalues frequency and damping can be graphically represented against the speed, making use for example of the international standard atmosphere (ISA) to relate dynamic pressure and Mach to altitude and speed, in what is traditionally called a vg-vf plot.

As an additional note, it needs to be mentioned that the eigenvalues are only valid at the existing flutter points for each eigenvector $(\mathrm{g}=0)$ since this is the assumption used to generate the aerodynamic forces. In spite of this, the $\mathrm{p}-\mathrm{k}$ method is commonly accepted in the industry.

The predicted flutter dynamic pressure for the system, using Theodorsen's formulation without Prandtl-Glauert compressibility correction for the aerodynamic forces and the p-k method, is calculated to be approximately at sea-level at $\mathrm{M}=0.2$ and around $4500 \mathrm{~m}$ for $\mathrm{M}=0.3$, as represented in figure 5.22 .

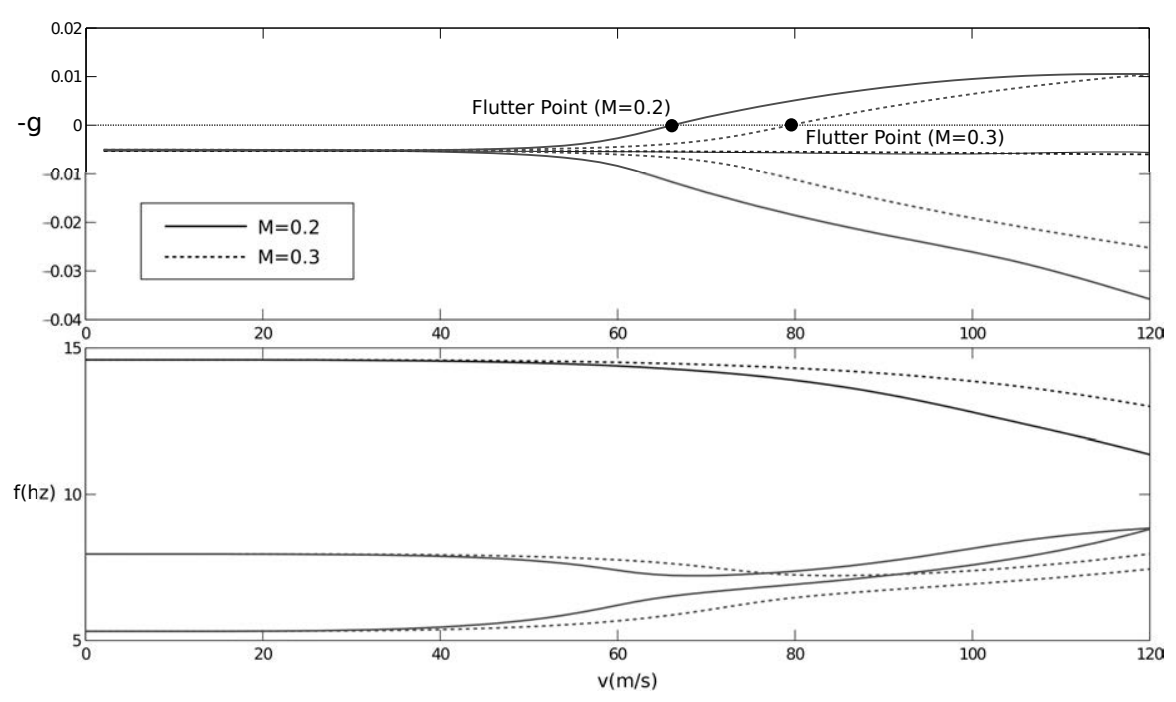

Figure 5.22: Frequency and damping evolution with incoming flow sped of the aeroelastic system (vg-vf flutter plot) at $\mathrm{M}=0.2$ and $\mathrm{M}=0.3$ 


\subsubsection{Results for the subsonic flow at $M=0.2$}

As a first case to test the adaptive POD on the fly methodology for aeroelastic simulations, we consider the impulse response of the structure in the low subsonic regime, $\mathrm{M}=0.2$.

At the light of the forced oscillation results, the POD modes have been calculated using the steady state as reference, and the ROM residuals have been minimized using a Gauss-Newton algorithm. The tunable parameters of the problem appearing in $\S 2.3 .2$ are selected for the subsonic case considered as

$$
T_{0}^{\mathrm{CFD}}=0.05 \mathrm{~s}, \quad \Delta T^{\mathrm{CFD}}=0.05 \mathrm{~s}, \quad \epsilon_{1}=10^{-10}, \quad K=100 .
$$

The aeroelastic simulations are run at a speed below (figure 5.23) and above (figure 5.24) the critical flutter speed. As in the forced oscillation case, the unshaded areas in both figures represent time spans at which the ROM has lost its validity, under the criteria presented above, and the full numerical solver has been run for a time $\Delta T^{\mathrm{CFD}}$ to update the modal basis. As it can be seen, the agreement is excellent for the initial phases of the simulation but the control surface rotation displacement deviates slowly from the numerical solver results as the simulation evolves, indicating that the update criteria would ideally need to be made more stringent. Additional runs show that just by doubling the value of $K$, the agreement is excellent for the whole simulation (see figure 5.25). The sensitivity to the value of $K$ is further explored in §5.4.2.

One additional interesting remark is the fact that for both simulation points very few updates are deemed necessary once the ROM has acquired a sufficient number of modes.

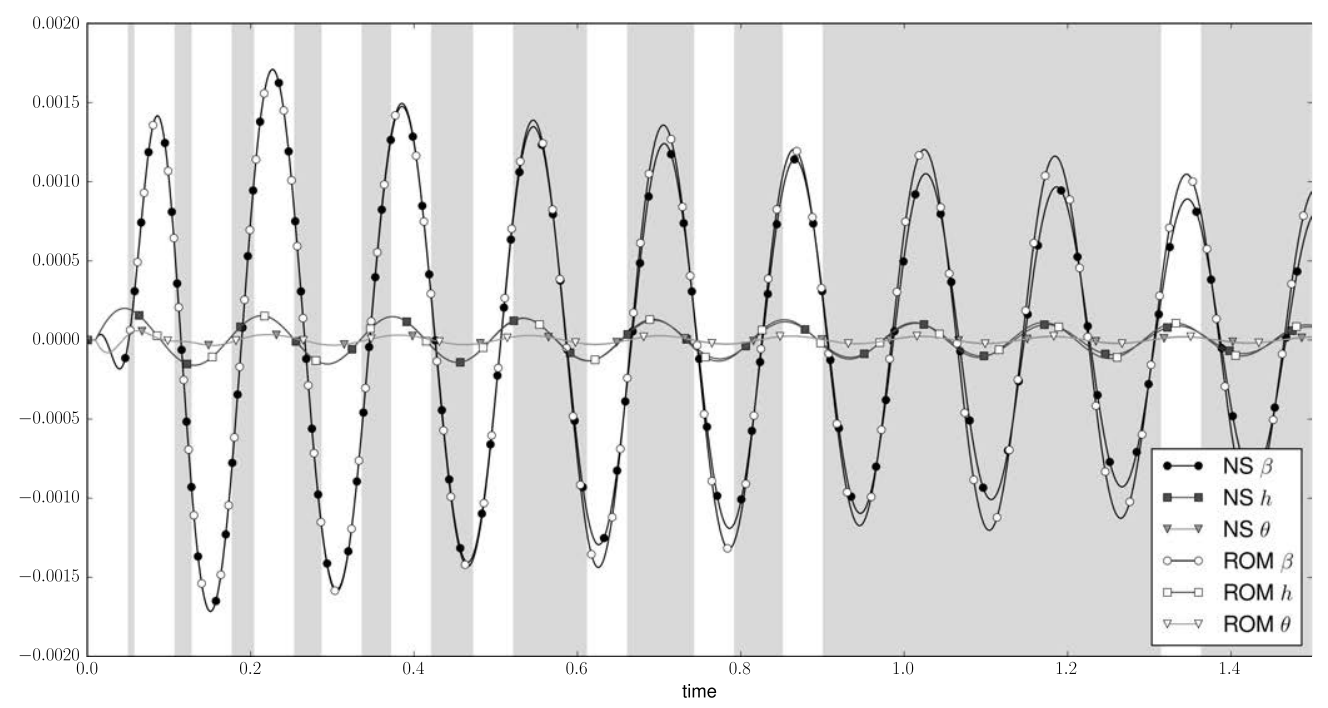

Figure 5.23: POD on the fly vs. the CFD solver. $M=0.2$ at a speed below flutter point 


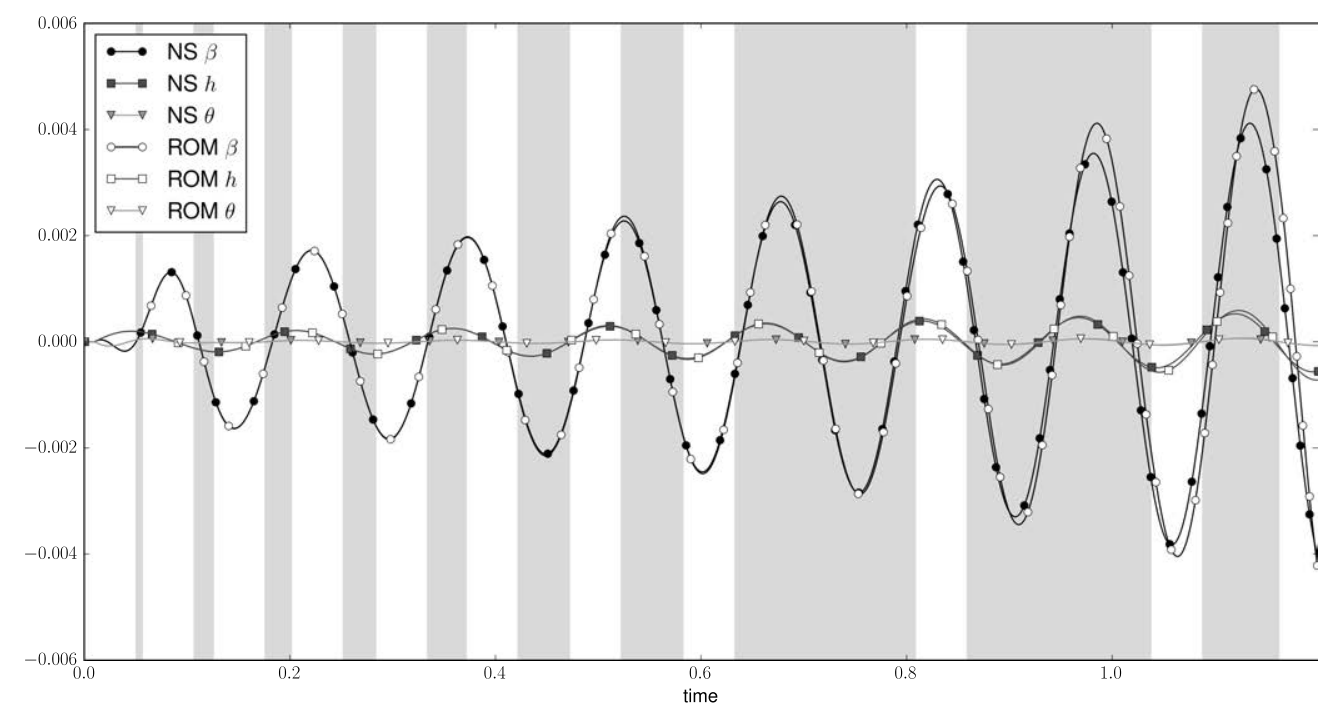

Figure 5.24: $\mathrm{POD}$ on the fly vs. the CFD solver. $\mathrm{M}=0.2$ at a speed above flutter point

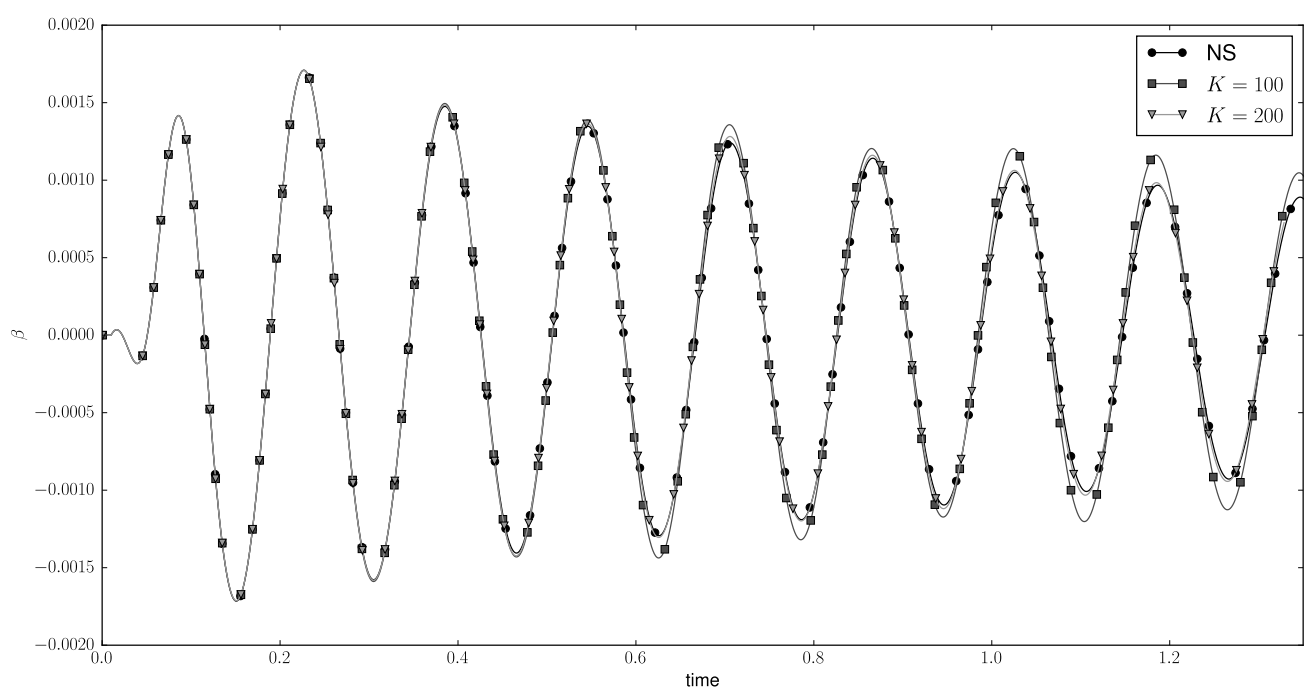

Figure 5.25: $K$ effect on control surface rotation, for $\mathrm{M}=0.2$ at a speed below flutter point 


\subsubsection{Results for the transonic flow at $\mathrm{M}=0.8$}

Let us now increase the Mach number to $M=0.8$. As shown for the forced oscillation case, the NACA0010 airfoil exhibits strong shocks in the pressure and suction sides of the airfoil, which need to be captured by the ROM (see for example figure 5.28)

At the light of the forced oscillation results, the POD modes have been calculated using the steady state as reference, and the ROM residuals have been minimized using a Gauss-Newton algorithm. As for the forced oscillation case, the ROM updates have been made more frequent, by reducing the value of $\Delta T^{\mathrm{CFD}}$. The tunable parameters of the problem appearing in $\S 2.3 .2$ are selected for the transonic case considered here as

$$
T_{0}^{\mathrm{CFD}}=0.05 \mathrm{~s}, \quad \Delta T^{\mathrm{CFD}}=0.005 \mathrm{~s}, \quad \epsilon_{1}=10^{-10}, \quad K=100 .
$$

The aeroelastic simulations are run at a speed below (figure 5.26) and above (figure 5.27) the critical flutter speed. Overall, the agreement is excellent, with both simulations practically indiscernible from each other. This comes, however, at the cost of more frequent ROM updates needed to capture the significant shock movement.

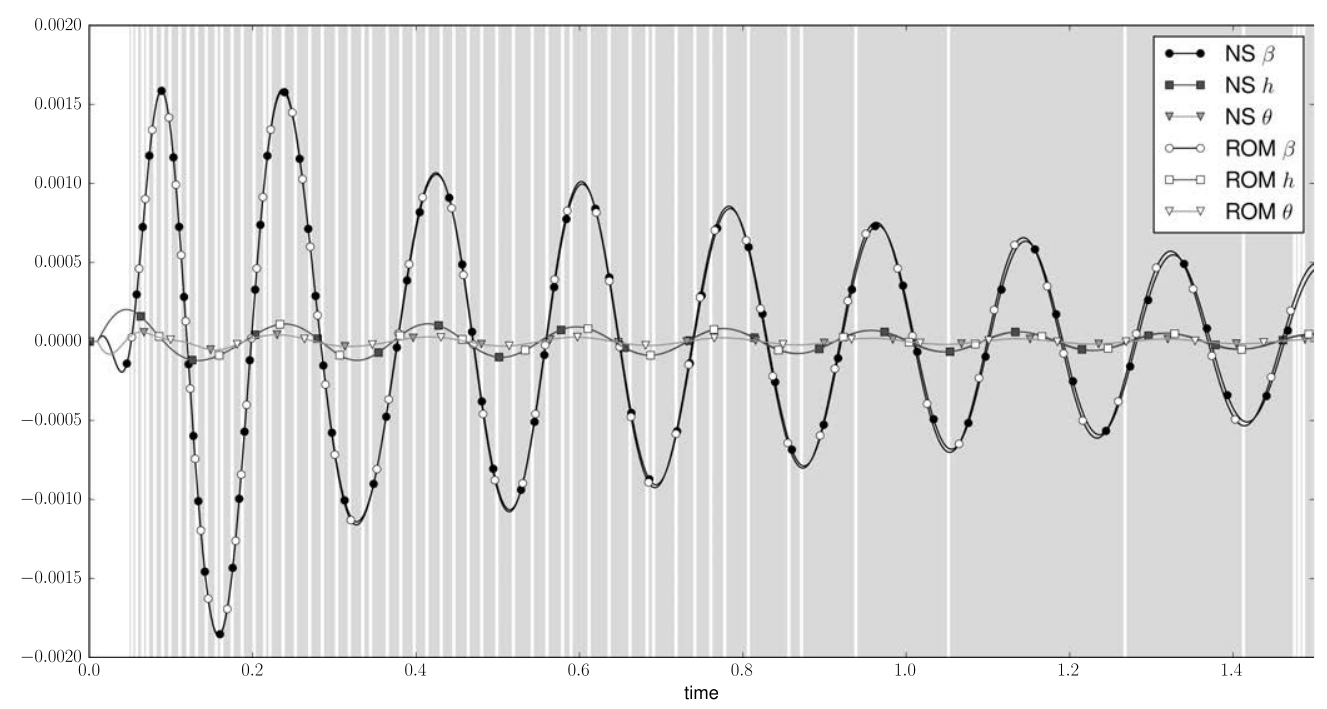

Figure 5.26: POD on the fly vs. the CFD solver. $M=0.8$ at a speed below flutter point 


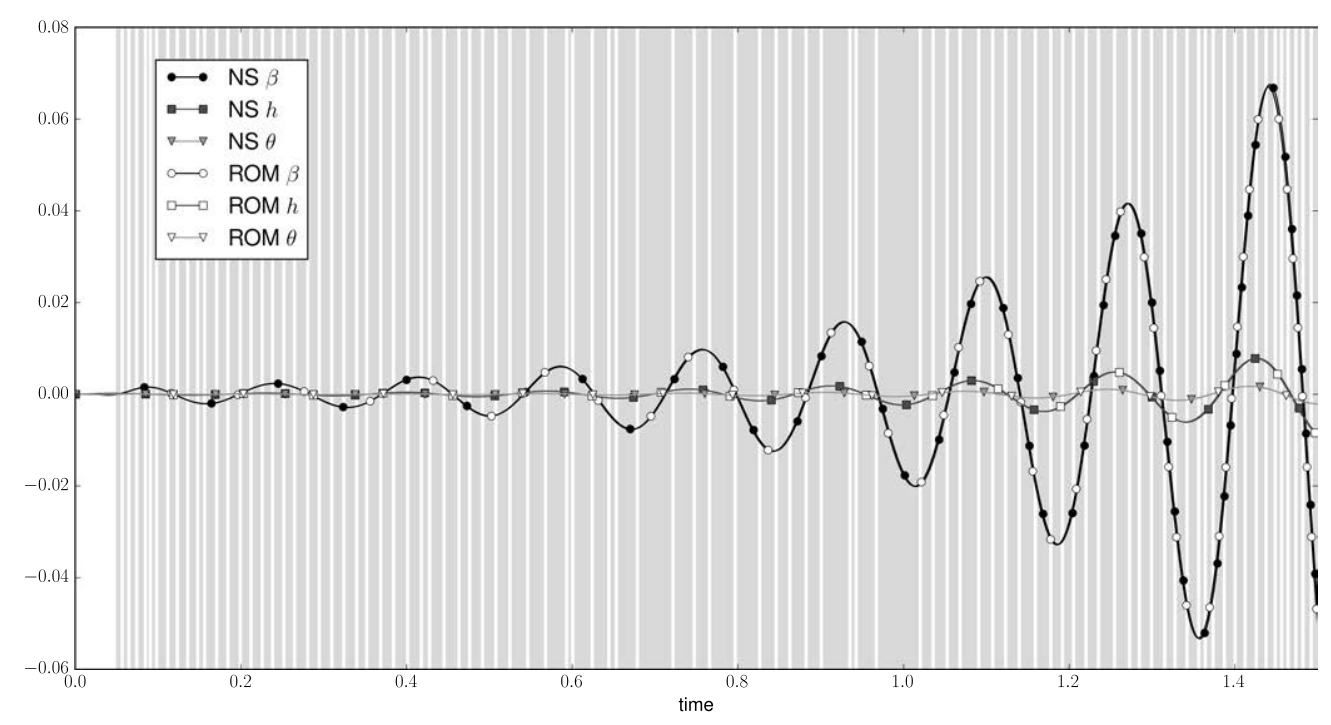

Figure 5.27: POD on the fly vs. the CFD solver. $\mathrm{M}=0.8$ at a speed above flutter point

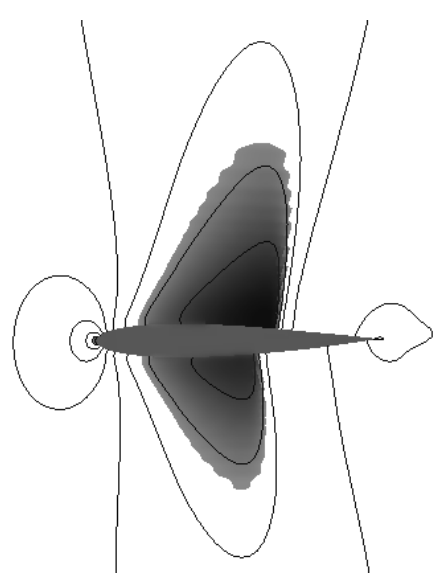

Figure 5.28: Iso Mach contours during simulation at $\mathrm{M}=0.8$ 


\subsection{Remarks on ROM efficiency}

\subsubsection{ROM efficiency and number of modes}

The ratio between the time the POD on the fly ROM is run and the time the full solver is run, in order to update the modal basis, is presented here as a first measure of the ROM efficiency.

For the subsonic case (figure 5.29), this ratio increases as the simulation time (and the number of restarts) increases, up to the point that almost no more restarts are needed. In the transonic case (figure 5.30) we have two distinct effects. For the stable and unstable cases, the ratio starts growing (up to very high values for the stable case) but then the ratio decreases mainly due to the fact that for the unstable case the amplitude keeps growing, and the shock movement increases.

The previous indicator neglects the fact that the computational cost of the ROM is not negligible. Instead, it increases as the number of retained modes increases. Thus, it is convenient to also take into account the required number of POD modes that are retained. As shown, the number of modes increases with the simulation time, converging to a given number for each of the considered conditions. For the unstable transonic case, since the distance the shock travels increases with each oscillation, the number of modes needed keeps increasing.

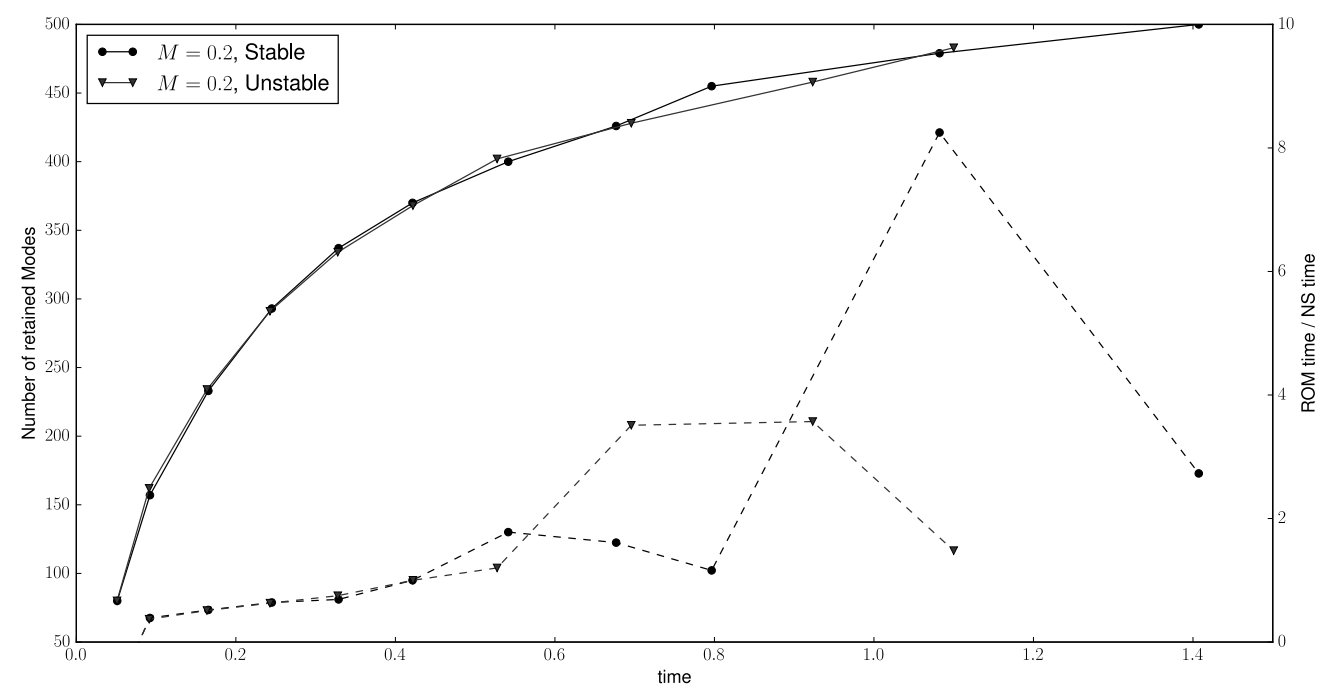

Figure 5.29: Number of retained modes (-) and ROM vs NS times ratio (-- ) for the $\mathrm{M}=0.2$ aeroelastic cases

\subsubsection{Solution method and tunable parameters effects}

In the previous sections, the selection of parameters and the solution method used has been highlighted. Let us turn now the attention to the effect of different solution methods and parameters on the ROM efficiency. For this, the efficiency of the ROM for different values and solution strategies will be evaluated in the subsonic case. 


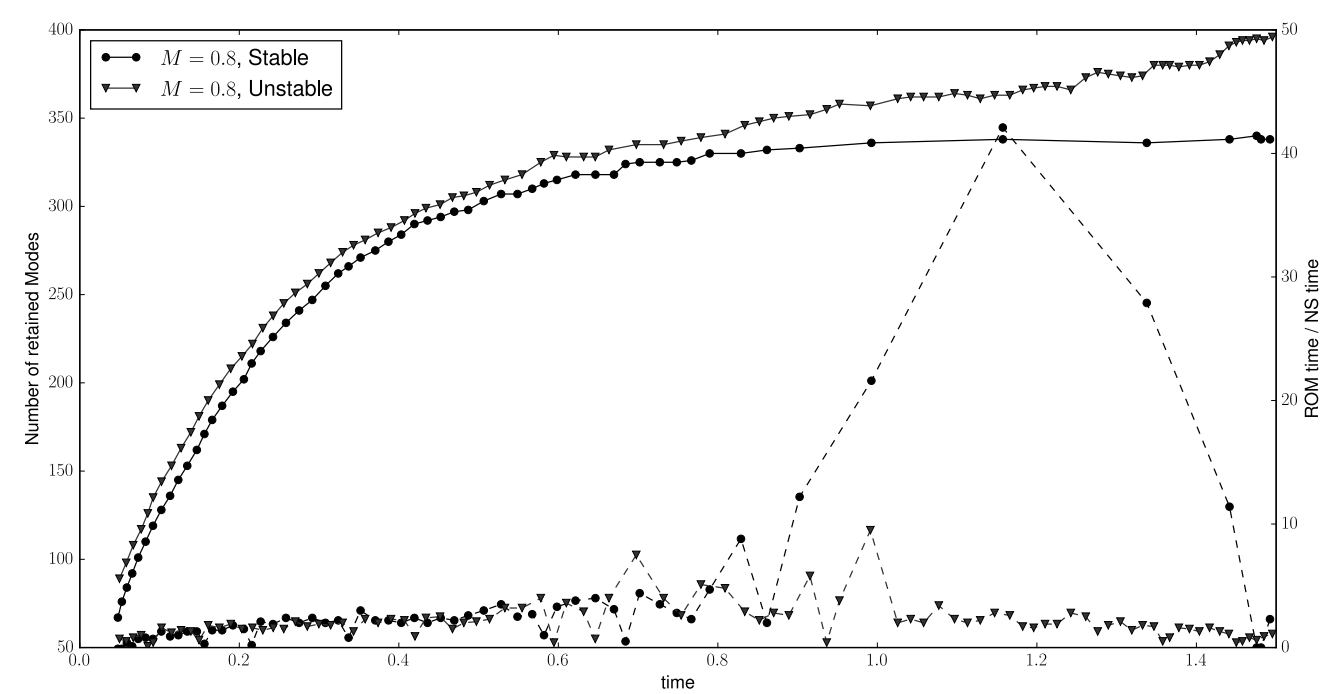

Figure 5.30: Number of retained modes (-) and ROM vs NS times ratio (-- ) for the $\mathrm{M}=0.8$ aeroelastic cases

\section{Effect of $K$}

As mentioned above for the subsonic case, it was needed to increase the value of $K$ in order to have a better approximation to the solution obtained with the numerical solver. Apart from limiting the validity of the ROM, its effect on the ROM efficiency and number of modes needs to be explored. The figure 5.31 shows that, not only doubling the value of $K$ results in an improvement of the approximation, but also, since the ROM solution does not depart from the full NS solution, the efficiency is also increased for approximately the same number of modes.

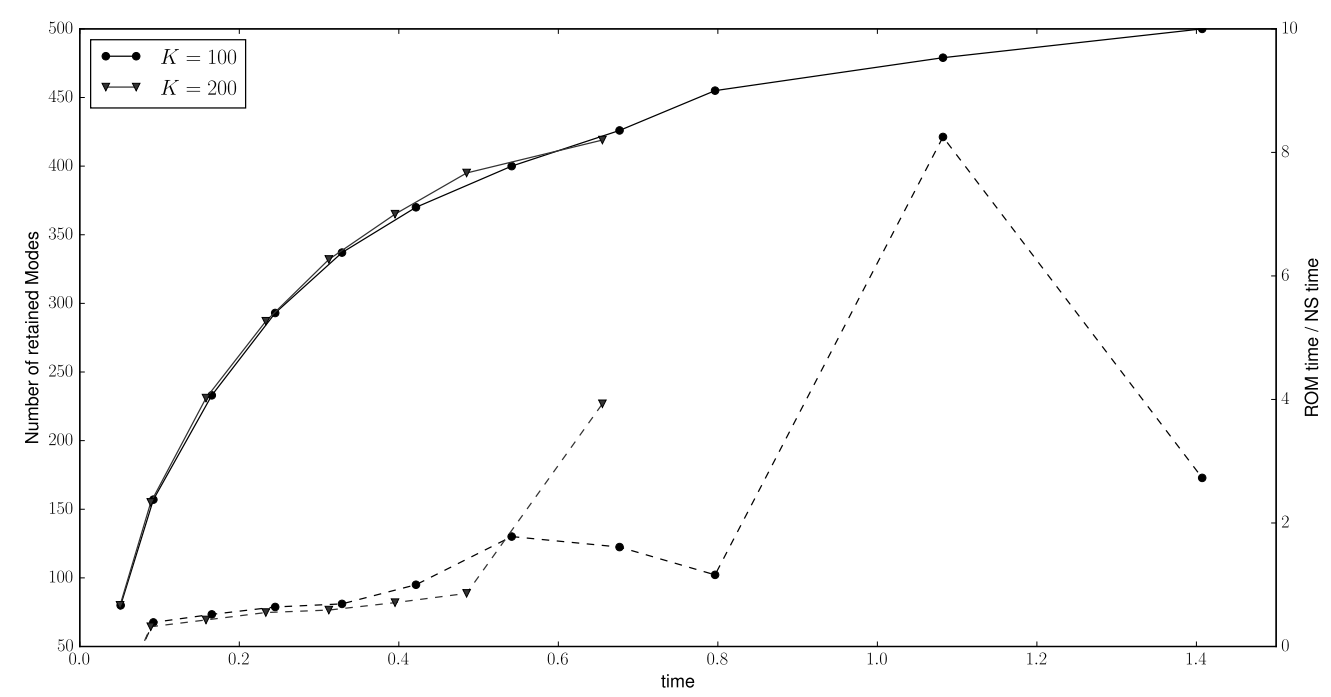

Figure 5.31: $K$ Effect on the number of retained modes $(-)$ and ROM vs NS times ratio (--) for the $\mathrm{M}=0.2$ stable case. Note that for $K=100$ no more updates are needed and, subsequently, no data points are available 


\subsection{Conclusions}

The extension of the novel POD on the fly method to the simulation of unsteady aerodynamics, and its application to aeroelastic simulations, has been successfully demonstrated in the cases where fluid viscosity is neglected. The lack of viscosity in the simulation has posed some additional challenges in the extension of the method, which have been resolved to obtain a robust method with high application potential. Several new ingredients have been incorporated additionally to the method, such as the ability to deal with deforming meshes and shock motion.

In order to demonstrate the application of the method, a two-dimensional airfoil has been considered, in contrast with the more computationally expensive, and expected to have higher gains, three-dimensional lifting surface.

The method has shown significant accelerations for the subsonic case both in the case of forced motions (pitching and heave oscillations) and impulse response, to the point that after a given point the ROM is capable of taking over the full simulation, reverting seldom to the full solver. In the transonic case the method has to deal with the motion of the strong shocks present on the airfoil, which imposes a shorter validity of the ROM, but the ROM is still capable of significant performance increases.

Several sensitivities to the tunable parameters available for the simulations have also been tested and analyzed. The minimization of the ROM residual in a least square sense has been proven to be the most robust method that allows to evolve the ROM in time. The use of a reference for the snapshot and the projection is closely linked to the error allowed in the reconstruction $\left(\varepsilon_{1}\right)$ and good results can be obtained for either method, proving once more its robustness.

A further increase in the computational efficiency may result by using generic mode libraries (Rapún et al., 2015), which highly reduces the required value of $\Delta T^{\mathrm{CFD}}$ (thus, increasing the overal efficiency of the method) when simulating very complex dynamics in the Complex Ginzburg-Landau equation. 


\section{Chapter 6}

\section{Turbulent viscous flow simulations}

The extension of the POD on the fly method to inviscid unsteady aerodynamics and dynamic aeroelastic simulations has been successfully performed in the previous chapter. Let us turn our attention to the simulations in the presence of viscosity at high Reynolds numbers. As mentioned, the absence of viscosity was a challenge for the inclusion of the ROM solver in the inviscid simulations. However, the inclusion of viscosity in the numerical solver adds significant challenges to the convergence of the numerical solver itself, some of which have not completely been solved. Therefore, the current results, although still acceptable and capable of showing the potential of the method, should be treated as preliminary.

There are two new main ingredients required to successfully simulate turbulent viscous flows at high Reynolds number: The addition of turbulence models (SpalartAllmaras in this work) and the consequent required increase in the spatial discretization accuracy.

This chapter introduces first the implementation details in the numerical solver, namely turbulence model and the addition of second order accurate spatial discretization schemes, and continues with the necessary adaptations required by the ROM solver in order to treat adequately such viscous simulations. Finally, the method is exercised by carrying out similar analyses as the ones presented for the inviscid case for an airfoil.

As an initial step prior to perform any simulation, the CFD code has been validated for viscous simulations (see Appendix D).

\subsection{Additions to the numerical solver for simulations of tur- bulent flow}

\subsubsection{Turbulence model}

In order to calculate the effective viscosity in the diffusive terms, which under the Boussinesq assumption constitute the Reynolds stress tensor, the formulation needs to be closed with a turbulence model. The one equation modified Spalart-Allmaras model (Allmaras and Johnson, 2012) has been used in this work, since it provides 
additional accuracy over algebraic models. In addition, it also avoids the complications involved in implementing algebraic models on unstructured meshes, is reasonably robust and inexpensive, and has been shown to yield favorable results in three-dimensional aerodynamic flows (Mavriplis, 1995).

The baseline S-A model (Spalart and Allmaras, 1992), without tripping terms, calculates the eddy viscosity $\nu_{t}$ as

$$
\nu_{t}=\frac{\mu_{t}}{\rho}=\tilde{\nu} f_{v_{1}}, \quad f_{v_{1}}=\frac{\chi^{3}}{\chi^{3}+c_{v_{1}}^{3}}, \quad \chi \equiv \frac{\tilde{\nu}}{\nu}
$$

where $\nu$ is the kinematic viscosity, $\nu_{t}$ is the turbulent kinematic viscosity and $\mu_{t}$ is the turbulent molecular viscosity. $\tilde{\nu}$ is the S-A auxiliary working variable, which obeys the transport equation

$$
\frac{\partial \tilde{\nu}}{\partial t}+\nabla \cdot \tilde{\nu}=P-D+T+\frac{1}{\sigma}\left[\nabla \cdot((\nu+\tilde{\nu}) \nabla \tilde{\nu})+c_{b_{2}}(\nabla \tilde{\nu})^{2}\right]
$$

$P$ and $D$ correspond to production and wall destruction terms respectively, and are given by

$$
P=c_{b_{1}} \tilde{S} \tilde{\nu}, \quad D=\left(c_{w_{1}} f_{w}\right)\left[\frac{\tilde{\nu}}{d}\right]^{2} .
$$

Here, $\tilde{S}$ is the modified vorticity,

$$
\tilde{S} \equiv S+\frac{\tilde{\nu}}{\kappa^{2} d^{2}} f_{v_{2}}, \quad f_{v_{2}}=1-\frac{\chi}{1+\chi f_{v_{1}}}
$$

where $S \equiv|\nabla \times \bar{u}|$ is the magnitude of the vorticity, and $d$ is the distance to the closest wall. Additionally, the function $f_{w}$ corresponds to

$$
f_{w}=g\left[\frac{1+c_{w_{3}}^{6}}{g^{6}+c_{w_{3}}^{6}}\right]^{1 / 6}, \quad g=r+c_{w_{2}}\left(r^{6}-r\right), \quad r=\min \left(\frac{\tilde{\nu}}{\tilde{S} \kappa^{2} d^{2}}, r l i m\right)
$$

The tunable constants values have been assigned taking their nominal values into account, which are

$$
\begin{array}{lll}
c_{b_{1}}=0.1355 & \sigma=\frac{2}{3} & c_{b_{2}}=0.622 \\
\kappa=0.41 & c_{w_{1}}=\frac{c_{b_{1}}}{\kappa^{2}}+\left(\frac{1+c_{b_{2}}}{\sigma}\right) & c_{w_{2}}=0.3 \\
c_{w_{3}}=2 & c_{v_{1}}=7.1 & \text { rlim }=10
\end{array}
$$

Additionally, in an attempt to add robustness to the turbulence model, Allmaras and Johnson introduced (Allmaras and Johnson, 2012) the following modified form of $\tilde{S}$, such that it remains positive for all the values of $S$

$$
\bar{S}=\frac{\tilde{\nu}}{\kappa^{2} d^{2}} f_{v_{2}}
$$




$$
\tilde{S}= \begin{cases}S+\bar{S} & : \bar{S} \geq-c_{v_{2}} S \\ S+\frac{S\left(c_{v_{2}}^{2} S+c_{v_{3}} \bar{S}\right)}{\left(c_{v_{3}}-2 c_{v_{2}}\right) S-\bar{S}} & : \bar{S}<-c_{v_{2}}\end{cases}
$$

with $c_{v_{2}}=0.7$ and $c_{v_{3}}=0.9$.

The discretization of the terms involved in the S-A turbulence model is carried over from the fluid governing equations and is not expanded here.

Finally, the effective molecular viscosity and the effective thermal conductivity are calculated combining the laminar and turbulent terms as

$$
\mu_{e}=\mu+\mu_{t}, \quad k_{e}=\frac{\mu}{\operatorname{Pr}(\gamma-1)}+\frac{\mu_{t}}{\operatorname{Pr}(\gamma-1)}
$$

where $P r_{t}$ corresponds to the turbulent Prandtl number.

\subsubsection{High order convection scheme}

The inviscid simulations presented before have used the Kurganov-Noelle-Petrova central scheme with a first order approximation, in which the values of the $\mathrm{P}$ and $\mathrm{N}$ cells were directly assigned to the each side of the cell faces. During the validation phase of the turbulent viscous flow solver, it was seen that the numerical diffusion added by the scheme was high enough to prevent the development of turbulent boundary layer profiles in the vicinity of the body (see $\S D .1$ ). The introduction of a second order spatial differencing scheme has been seen to solve the issue, but produces unphysical oscillations that require the use of flux limiters, included already in the original development of the KNP central scheme (Kurganov et al., 2001). This section will show the inclusion of flux limiters in the unstructured cell centered flow solver.

Godunov's theorem (Wesseling, 2009) proves that linear schemes which preserve monotonicity are, at most, only first order accurate (Hirsch, 2007). In other words, a scheme of order larger than one needs to be non-linear, i.e. it depends on the local shape of the solution, in order to be bounded and more than first-order-accurate at the same time (Jasak, 1996).

Following Sweby (Sweby, 1984), a second order scheme approximation of the face values for a general variable $\phi$ is written as a combination of the first order bounded differencing scheme (UD) and a high order correction (HO) using a limiter $\beta$, as

$$
\phi_{f}=\phi_{U D}+\beta\left(\phi_{H O}-\phi_{U D}\right)
$$

where $\beta$ is a function of $r$, the successive gradients of the convected variable (Van Leer, 1974). Assuming that the cells are oriented in the direction of the flow, as seen in figure 6.1, we have that

$$
r=\frac{\phi_{i}-\phi_{i-1}}{\phi_{i+1}-\phi_{i}}
$$

Furthermore, Sweby showed that if $\beta(r)$ lies in the shaded region of figure 6.2 the 


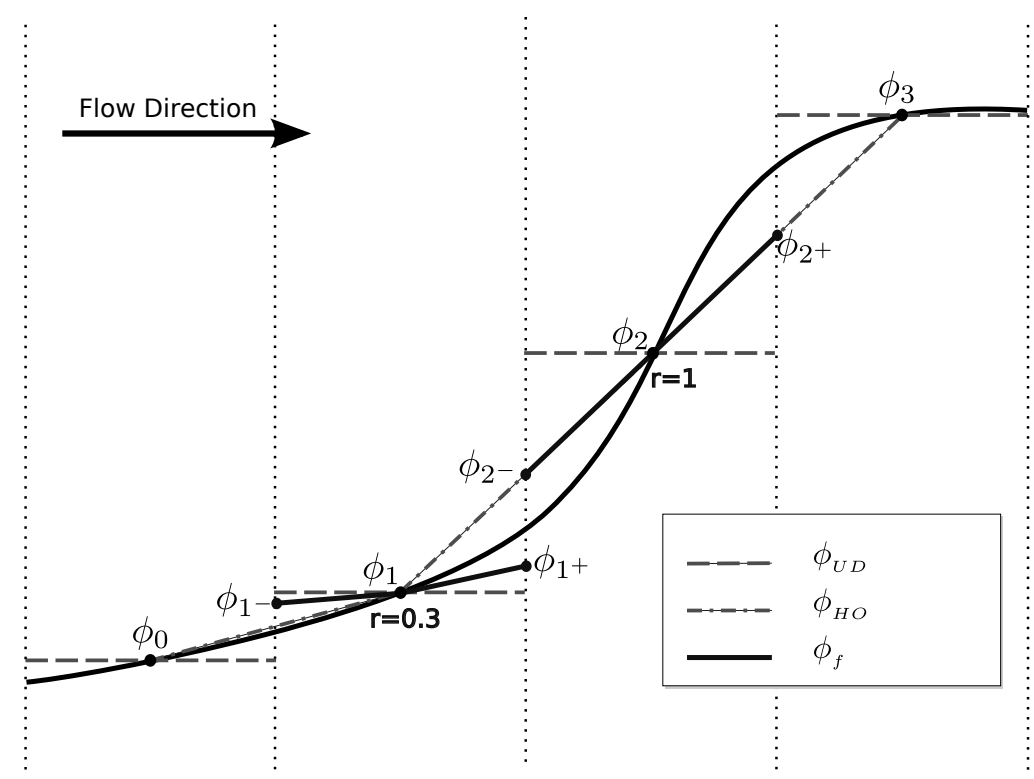

Figure 6.1: Variable succesive gradients and Limiter effect on the scheme's face values

scheme is second order accurate and satisfies the TVD (total variation diminishing) condition (Harten, 1983), such that if the total variation of the solution is defined as

$$
T V\left(\phi^{n}\right)=\sum_{f}\left|\phi_{N}^{n}-\phi_{P}^{n}\right|
$$

then, for every time step the solution fulfills

$$
T V\left(\phi^{n+1}\right) \leq T V\left(\phi^{n}\right)
$$

The TVD boundedness criterion derived by Sweby depends upon the Courant number, meaning that the solution would, strictly speaking, depend on the simulation time step. Despite this known deficiency, second order accurate TVD schemes are widely used for practical applications and have been selected for the work presented in this thesis. In particular, the Van Leer limiter, first expressed by Van leer (Van Leer, 1974) and adapted by Sweby (Sweby, 1984), has been used due to the good results shown in conjuction with the KNP and KT schemes (Greenshields et al., 2010). The shape of the limiter is plotted in figure 6.2 on top of Sweby's diagram, showing that it fulfills the requirement to be second order accurate and TVD. It can be expressed as

$$
\beta(r)=\frac{|r|+r}{1+|r|}
$$

It can also be easily seen that the limiter is symmetric, such that $\frac{\beta(r)}{r}=\beta\left(\frac{1}{r}\right)$, ensuring that the limiting actions for forward and backward gradients operate in the same way.

For unstructured meshes, the ratio of successive gradients can not be expressed using the values of the upstream and downstream consecutive cells, since these cells are not 


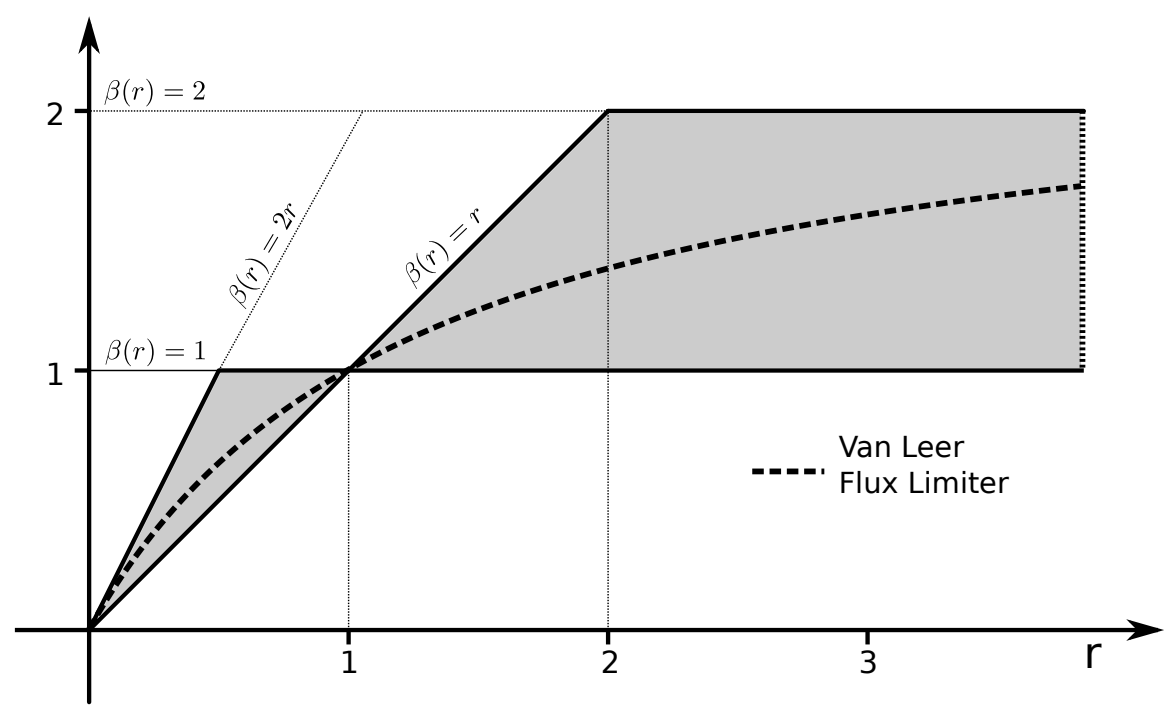

Figure 6.2: Sweby's diagram and Van Leer limiter

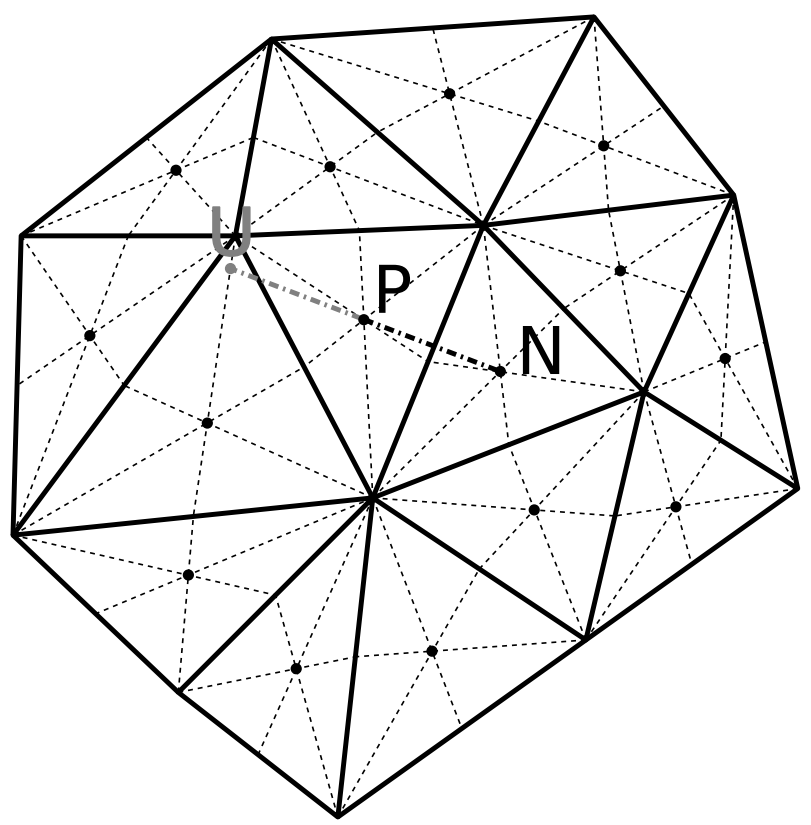

Figure 6.3: Typical triangular unstructured mesh with the presence of a virtual cell to estimate the ratio of successive gradients

generally known, and might not even exist. In this case, it is necessary to make use not only of the values at each cell center, but also of the gradients of the variable. Thus, we can construct a virtual node U (Darwish and Moukalled, 2003), representing the node upstream of node $\mathrm{P}$, as in figure 6.3 , so that referencing the nodes $i-1, i, i+1$ in the $1 D$ structured case as $U, P$ and $N$, respectively, it follows that

$$
r=\frac{\phi_{P}-\phi_{U}}{\phi_{N}-\phi_{P}}=\frac{\left(\phi_{N}-\phi_{U}\right)-\left(\phi_{N}-\phi_{P}\right)}{\phi_{N}-\phi_{P}}=\frac{\phi_{N}-\phi_{U}}{\phi_{N}-\phi_{P}}-1 .
$$


The $\phi_{U}$ values are replaced taking into account that

$$
\left(\phi_{N}-\phi_{U}\right)=\nabla \phi_{P} \cdot \overrightarrow{r_{U N}}=2 \nabla \phi_{P} \cdot \overrightarrow{r_{P N}}
$$

where $\overrightarrow{r_{P N}}$ is the vector joining the nodes $\mathrm{P}$ and $\mathrm{N}$. The position of the node $\mathrm{U}$ is chosen such that it lies along the line joining both cell centers that share the face, namely $\mathrm{P}$ and N. Other positions of $U$ could also be chosen, but with loss of accuracy as the cell gradient yields a second order accuracy only when the difference is centered around node P. Finally, the formulation of $r$ used in this work for unstructured meshes is represented as

$$
r=\frac{2 \nabla \phi_{P} \cdot \overrightarrow{r_{P N}}}{\phi_{N}-\phi_{P}}-1
$$

Combining the limiter with the linear interpolation scheme presented in equation (3.16) and naming $\phi_{P f}$ and $\phi_{N f}$ the values of the variable at the $\mathrm{P}$ and $\mathrm{N}$ sides of the face, we finally have

$$
\begin{array}{r}
\phi_{P f}=\phi_{P}\left(1-\beta\left(1-w_{f}\right)\right)+\phi_{N} \beta\left(1-w_{f}\right) \\
\phi_{N f}=\phi_{P} \beta w_{f}+\phi_{N}\left(1-\beta w_{f}\right)
\end{array}
$$

\subsubsection{Flow solver algorithm}

In principle, the inclusion of the turbulence auxiliary variable would not require any modifications to the solution algorithm of the flow governing equations, and would just require the addition of the relevant rows to the residuals $\mathcal{R}$ and fluid state variables $\mathcal{Q}$ in equation (2.3). Unfortunately, the slow convergence of the turbulence equation jeopardizes the overall solution, while increasing the size of the Jacobian and the overall cost.

Following a similar approach as in other codes (e.g. CFL3D), the turbulence equations are solved decoupled from the mean flow equations in the following way:

1. While keeping the turbulent viscosity and turbulent thermal conductivity constant, the mean flow equations are iterated for a given number of $t-\tau$ sub-iteration steps. The Laplacian terms are treated implicitly, while the orthogonal correction and gradient related terms are treated explicitly using the values from each previous sub-iteration. The exact Jacobian from the first sub-iteration is kept constant for the remaining sub-iterations.

2. The turbulence auxiliary variable is iterated using also a given number of $t-\tau$ sub-iteration steps while keeping the mean flow constant. The Laplacian terms are treated implicitly while the orthogonal correction, gradient related terms and nonlinear terms (like $\tilde{\nu}^{2}$ ) are treated explicitly using the values from each previous sub-iteration. An approximate Jacobian neglecting the nonlinear gradient terms, is built for the first sub-iteration and kept constant for the remaining subiterations. 
3. The turbulent viscosity and conductivity are calculated and added to form the effective viscosity and thermal conductivity terms.

4. The process is taken back to the first step until sufficient overall convergence is achieved.

\subsubsection{Numerical solver validation}

The presented additional ingredients to the numerical solver have been validated against several of the turbulence benchmark cases published by NASA (Rumsey, 2016). The validation results against the $2 \mathrm{D}$ Zero pressure gradient flat plate (2DZP), the 2D Airfoil near wake (2DANW), the 2D NACA 0012 airfoil (2DN00) and the 2D NACA 4412 airfoil trailing edge separation (2DN44) cases can be seen in Appendix D. Comparison against transonic available data for the 2D NACA 0012 airfoil as well as against the unsteady data generated with the already validated inviscid code are also presented in Appendix D.

With the presented results, the code has been deemed suitable to demonstrate the impact of the incorporation of the adaptive POD based ROM into the unsteady aerodynamic simulations for viscous turbulent flow.

\subsection{Forced harmonic oscillation of a NACA0012 airfoil}

The turbulent POD on the fly ROM has been exercised in an analogous way as in the inviscid solver. The unsteady two-dimensional flow around an airfoil subject to either pitch or heave oscillations is considered. Subsonic $(M=0.3)$ and transonic $(M=0.8)$ flow conditions at Reynolds $610^{6}$ have been selected.

Based on the good results for the inviscid case, only simulations involving deformation of the computational mesh are considered. That is, for these cases the farfield is kept stationary and the mesh deformed to conform to the rigid oscillating airfoil, even if the simulations could have been performed moving the numerical mesh rigidly with the body.

\subsubsection{Computational fluid domain model}

The aerodynamic shape has been chosen, without loss of generality, as a 4 digits symmetric, uncambered, NACA airfoil with a $12 \%$ thickness to chord length ratio, known as NACA-0012 (Garrick, 1933). The definition of the NACA 0012 airfoil is slightly altered from the original definition so that the airfoil closes with a sharp trailing edge. To do this, the following shape definition (Rumsey, 2016) has been used: 


$$
\begin{array}{r}
y_{t}=0.594689181 c\left[0.298222773 \sqrt{\frac{x}{c}}-0.127125232 \frac{x}{c}-0.357907906\left(\frac{x}{c}\right)^{2}\right. \\
\left.+0.291984971\left(\frac{x}{c}\right)^{3}-0.105174606\left(\frac{x}{c}\right)^{4}\right]
\end{array}
$$

where $c$ is the chord length, $x$ is the position along the chord (from 0 to $c$ ) and $y_{t}$ s the half thickness at a given value of $x$.

The mesh has been created following the recommendation from the Drag Prediction Workshop (Mavriplis et al., 2009). The far field boundaries are set such that a semi circular domain of radius 100 airfoil chords, with origin at $x=0.25 c$, defines the upstream boundary, and two 100 by 100 chords squares, whose corner has been smoothed, define the upper, lower and downstream boundaries. Finally, the domain is extruded to a thickness of 1 chord. A detail of the fluid domain can be seen in figure 6.4.

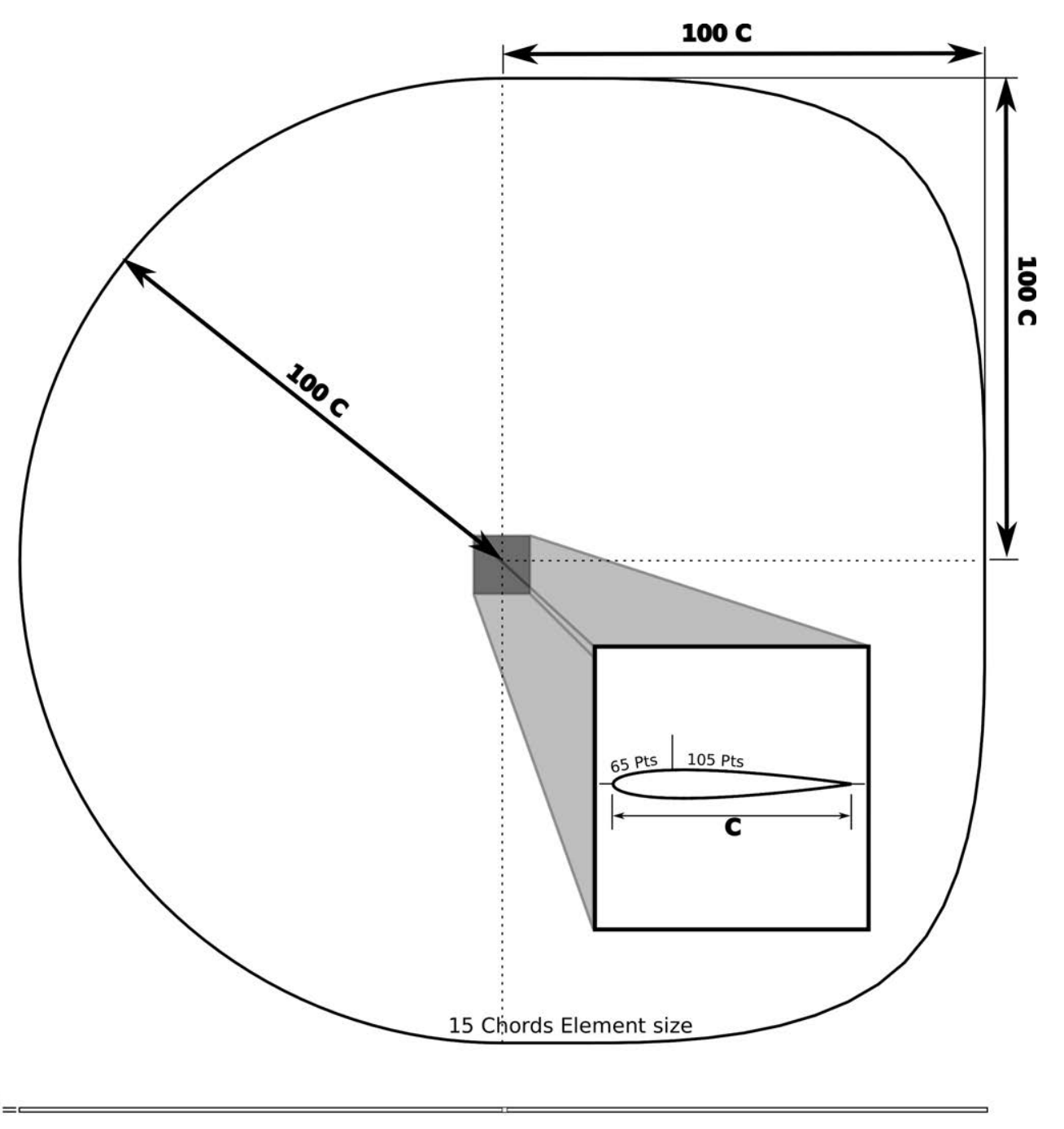

Figure 6.4: Airfoil mesh geometry 
The computational fluid domain has been generated in the boundary defined above using Gmsh (Geuzaine and Remacle, 2009), a three-dimensional finite element mesh generator with built-in pre- and post-processing utilities. First, a one-dimensional mesh seed is created along the boundaries (both internal and external); 65 points are distributed using a 0.95 distance growth rate in the two upstream quarters of the airfoil, and 105 points in the remaining two quarters (near the trailing edge), with 1.02 growth rate. The far field one-dimensional seed is created defining an element size of 15 chords.

In a second step, in order to allow the proper integration up to the wall of the turbulent flow, the airfoil mesh is extruded in the direction normal to the wall, creating a structured mesh. The first two cells are of the same height, set so that the distance of the first cell center to the wall is at a nondimensional wall distance $(\mathrm{y}+)$ close to 1 . The mesh is then extruded for 40 additional layers with a growth factor of 1.2. The remaining of the fluid domain between the last extruded layer and the farfield is filled by a unstructured Delaunay triangulation, forming what is known as a hybrid mesh, typically used by industrial unstructured solvers.

Finally, since this two-dimensional problem is to be solved using the three-dimensional CFD solver constructed as explained in Chapter 3, the two-dimensional mesh is extruded in the span direction to create the three-dimensional mesh in figure 6.5 consisting of 39134 points, 50333 faces, and 24975 cells.

Even if the mesh has some structured domains, the solver created for this work will treat it as unstructured, so no fixed pattern in the cells connectivity is assumed.

\subsubsection{Sub-mesh selection}

As in the inviscid case, the selection of the sub-mesh, in which the POD modes are going to be calculated and evolved, is crucial for the success and efficiency of the ROM time evolution. As a reminder, the selected cells act as the "master" cells, and the remaining just act, in some sense, as "slave" through the POD modes.

In order to adapt to unknown possible dynamics and shock motions, the same robust and simple approach as in the inviscid case has been chosen. A series of radial lines are traced at regular intervals from the airfoil wall to the far-field faces. Starting from the airfoil wall, the next selected cell is located at $0.0030 \mathrm{~m}\left(y^{+} \approx 1800\right)$, and the distance to the wall for the subsequent cells is obtained by geometric progression with a factor of 1.5. In the boundary layer extruded subdomain, it is straight forward to identify the selected cell. In the unstructured subdomain, the cell whose cell center is closest to the obtained distance, and aligned with the wall normal, is selected. Following this approach, $M_{o}=2053$ cells are selected.

One important issue that needs to be considered for the turbulent case is the number of retained cells. As mentioned, the POD modes are calculated for the selected cells, and the ROM is evolved minimizing the residuals of the selected cells, but the information of some other cells needs to be available in order to calculate the residuals. In the case of the inviscid simulations, the only necessary information was the corresponding values 


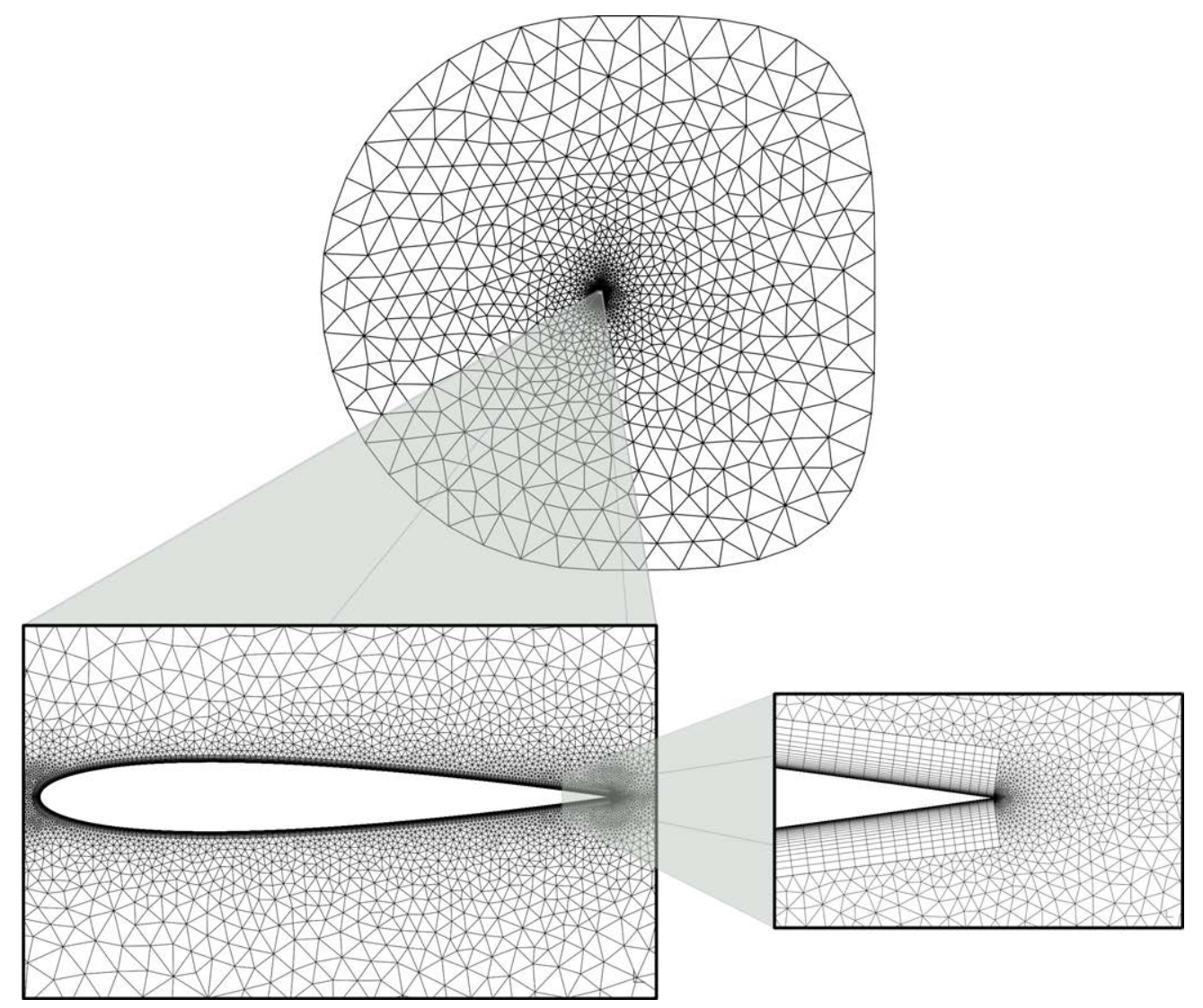

Figure 6.5: NACA0012 Airfoil mesh used for turbulent simulations

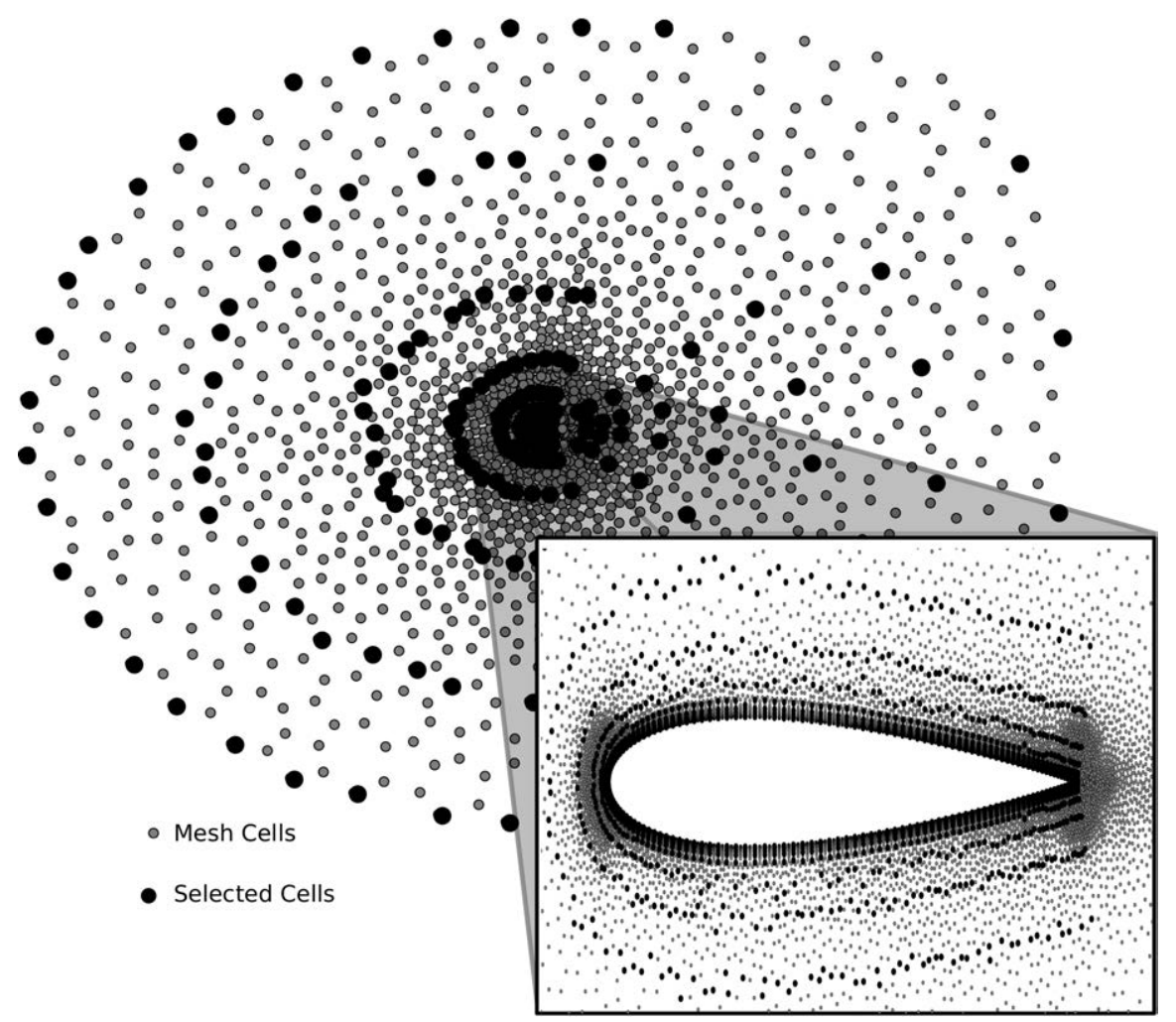

Figure 6.6: Sub-mesh selection 
of the neighboring cells, needed to calculate the gradients of the primitive variables. In the turbulent case, however, since the gradients of the neighboring cells are needed in order to implement the flux limiter presented in $\S 6.1 .2$, the values of the "neighbor's neighbor" of each selected cells need to be retained. This does not play a significant role in the simulation process, but increases the storage size in memory of each POD mode.

\subsubsection{ROM solution algorithm in the presence of turbulence model}

The addition of the turbulence model adds one or more additional variables, $\tilde{\nu}$ in our case for the Spalart-Allmaras turbulence model, but it could be different for other turbulence models (e.g. $k$ and $\omega$ or $k$ and $\epsilon$ for $k-\omega$ and $k-\epsilon$ turbulence models, respectively). There are several possible strategies to carry on the evolution of the turbulence variable during the ROM time evolution.

As in the inviscid case, the ROM evolution has been carried out by minimizing the least square residuals with a Gauss-Newton algorithm, with and without reference (null $\mathcal{Q}_{0}$ ). The Jacobian for the turbulence model has been approximated in such a way that only the diagonal terms are retained.

Several different approaches can be considered, as explained below.

\section{Augmented snapshot matrix}

The snapshot matrix, as described in equation (2.5) can be complemented with the turbulent auxiliary variable. Thus, naming $\mathcal{Q}_{t_{i}}=\{\rho, u, v, w, p, \tilde{\nu}\}_{i}$, the augmented snapshot matrix is defined as

$$
S=\left[\begin{array}{ccccc}
\mathcal{Q}_{t_{1}}^{1} & \mathcal{Q}_{t_{1}}^{2} & \cdots & \cdots & \mathcal{Q}_{t_{1}}^{T} \\
\mathcal{Q}_{t_{2}}^{1} & \mathcal{Q}_{t_{2}}^{2} & \cdots & \cdots & \mathcal{Q}_{t_{2}}^{T} \\
\vdots & \vdots & \vdots & \vdots & \vdots \\
\mathcal{Q}_{t_{M}}^{1} & \mathcal{Q}_{t_{M}}^{2} & \cdots & \cdots & \mathcal{Q}_{t_{M}}^{T}
\end{array}\right]
$$

From this snapshot matrix, the POD modes are calculated as in $\S 2.2 .1$ and the ROM solution is carried on in an equivalent way as for the inviscid case. This has, however, the inconvenient that in some regions of the flow field the value of $\tilde{\nu}$ is orders of magnitude higher than the values of the primitive variables. In this way, the POD modes would primarily try to minimize the error on the $S$ rows corresponding to $\tilde{\nu}$.

\section{Independent POD basis}

A way to solve the difference in orders of magnitude of the primitive and the auxiliary turbulence variable is to create an independent POD basis. For this, an additional snapshot matrix $\left(S_{\tilde{\nu}}\right)$ is created independently of the primitive variables snapshot matrix, 
defined as

$$
S_{\tilde{\nu}}=\left[\begin{array}{ccccc}
\tilde{\nu}_{1}^{1} & \tilde{\nu}_{1}^{2} & \ldots & \ldots & \tilde{\nu}_{1}^{T} \\
\tilde{\nu}_{2}^{1} & \tilde{\nu}_{2}^{2} & \ldots & \cdots & \tilde{\nu}_{2}^{T} \\
\vdots & \vdots & \vdots & \vdots & \vdots \\
\tilde{\nu}_{M}^{1} & \tilde{\nu}_{M}^{2} & \cdots & \cdots & \tilde{\nu}_{M}^{T}
\end{array}\right]
$$

From this snapshot matrix, an independent POD basis $\left(\Phi_{\tilde{\nu}}\right)$ is built, so that it can be used to evolve the turbulent model auxiliary variable in time during the ROM solution. This can be done in an analogous way to the full numerical solver, in which the turbulence model is solved loosely coupled from the primitive variables.

\section{The turbulence variable as "slave" to the primitive variables}

An additional approach can be taken if the snapshot matrix is built as in equation (6.20) but the selection based scalar product matrix $\mathcal{G}$ is created in such a way that the diagonal terms corresponding to the turbulence auxiliary variable are set to 0 . The resulting effect is that the turbulence variable in the complete fluid domain is made "slave" to the evolution of the primitive variables. In other words, the turbulence auxiliary variable is obtained in the fluid domain in the same way as the primitive variables are reconstructed in the non-selected cells. The evolution of the POD modes only depends on the projection of the mass, momentum and total energy equations into the POD modes.

This approach has been selected as the baseline in the examples below, since it is the one that best exemplifies the advantages of the method. That is, not only the values in one set of cells can be made slave to another set, but also one complete variable $(\tilde{\nu})$ can be made slave to a set of the primitive variables.

\subsubsection{Oscillatory forcing}

The unsteady flow field is forced by monochromatic pitch or heave oscillations of the airfoil, with either the angle of attack or the vertical position of the airfoil varying as $A o A=a[\cos (2 \pi f t)-1]$ or $h=a \cos (2 \pi f t$ ) (see figure 6.7). For convenience, the
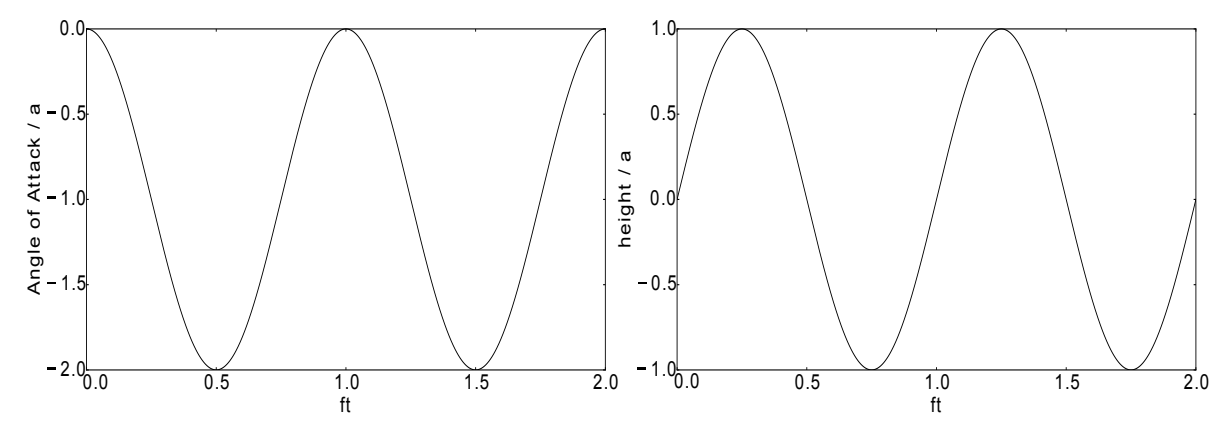

Figure 6.7: Rescaled forcing function for the pitch (left) and heave (right) motions, plotting the rescaled angle of attack and height vs. $f \cdot t$ 
reduced frequency (which is nondimensional and proportional to the Strouhal number), is defined in terms of the frequency $f$, the incoming velocity $u_{\infty}$, and the chord $c$ as

$$
k=\pi f c / u_{\infty}
$$

As anticipated, we consider pitch and heave motions with the following combinations of the forcing amplitude

$$
\text { pitch: } a=0.01 \text { and } 0.1 \mathrm{rad}, \text { heave: } a=0.05 c
$$

and two forcing frequencies, one giving a small value of the reduced frequency $k$ and another giving $k \sim 0.3$. Thus, we have six combinations for the forcing mode, which moreover will be considered in the next two subsections for a subsonic $(M=0.3)$ and a transonic $(M=0.8)$ case.

\subsubsection{Results for the time dependent subsonic flow at $M=0.3$}

As a first case to test the adaptive POD on the fly methodology, we consider the low subsonic regime, $\mathrm{M}=0.3$, at sea level altitude.

In an initial step, a steady simulation has been carried out to achieve the converged steady state. The unsteady simulation is started subsequently using the full numerical solver for a time $T_{0}^{\mathrm{CFD}}=0.05$, point at which the ROM takes over.

In the baseline approach, the turbulence auxiliary variable $\tilde{\nu}$ has been made slave to the primitive variables through the POD modes, as explained in §6.2.3. The POD modes are calculated without any reference (null $\mathcal{Q}_{0}$ ) using only the selected cells. The $\mathrm{ROM}$ residuals for the primitive variables, derived from the mean mass, momentum and energy conservation, are minimized in a least square sense (see equation (2.18)) making use of a Gauss-Newton algorithm. After convergence has been achieved, the flow field is reconstructed. If the condition from equation (2.21) is fulfilled for the primitive variables residuals, the ROM simulation is carried forward. In the opposite case, the solution is reverted back to the full NS solver.

The tunable parameters of the problem appearing in $\S 2.3 .2$ are selected for the subsonic case considered here as

$$
T_{0}^{\mathrm{CFD}}=0.05 \mathrm{~s}, \quad \Delta T^{\mathrm{CFD}}=0.020 \mathrm{~s}, \quad \epsilon_{1}=10^{-10}, \quad K=20
$$

For the pitch oscillations at the lowest forcing amplitude in equation (6.23) and the high forcing frequency, $f=10.0 \mathrm{~Hz}$, which in the present case gives a reduced frequency $k=0.32$, the outcome of the POD on the fly method is given in figure 6.8. Similarly to the previous chapter, the curves labeled NS are obtained using the CFD solver alone, while those labeled ROM are used combining the CFD solver and the low-dimensional ROM, using the POD on the fly method. The length of the time intervals during which the ROM is used (shaded region in the figure) steadily increases along the simulation, 
as it can also be seen in figure 6.9, meaning that the updating strategy described in $\S 2.3 .2$ is efficient to improve the POD modes as more information from the CFD solver (namely, a larger number of snapshots) is accounted for. As it can be seen, the forces produced on the airfoil with both methods are plot indistinguishable.

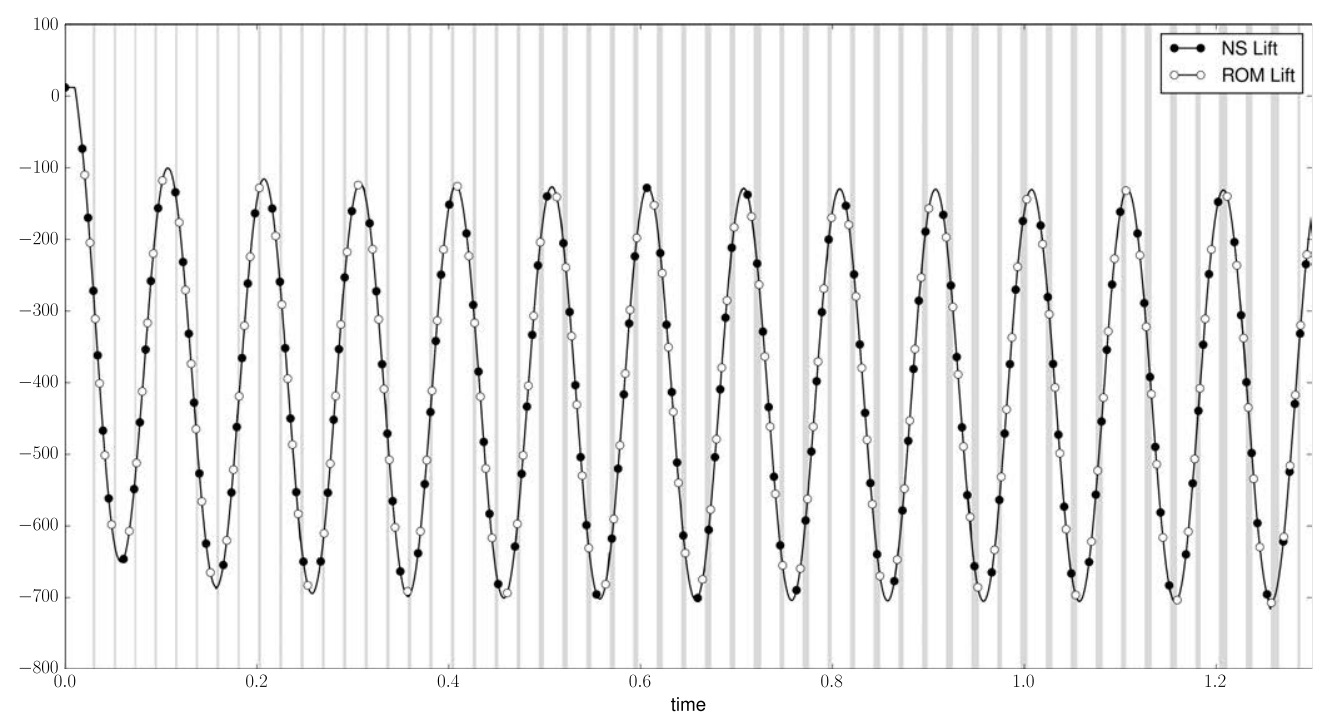

Figure 6.8: $\mathrm{POD}$ on the fly vs. the CFD solver, with $\mathrm{M}=0.3$ at sea level, considering pitch motion with $a=0.01 \mathrm{rad}$ and $f=10.0 \mathrm{~Hz}(k=0.32)$. Those intervals in which the low dimensional ROM is used in the POD on the fly method indicated with shaded regions

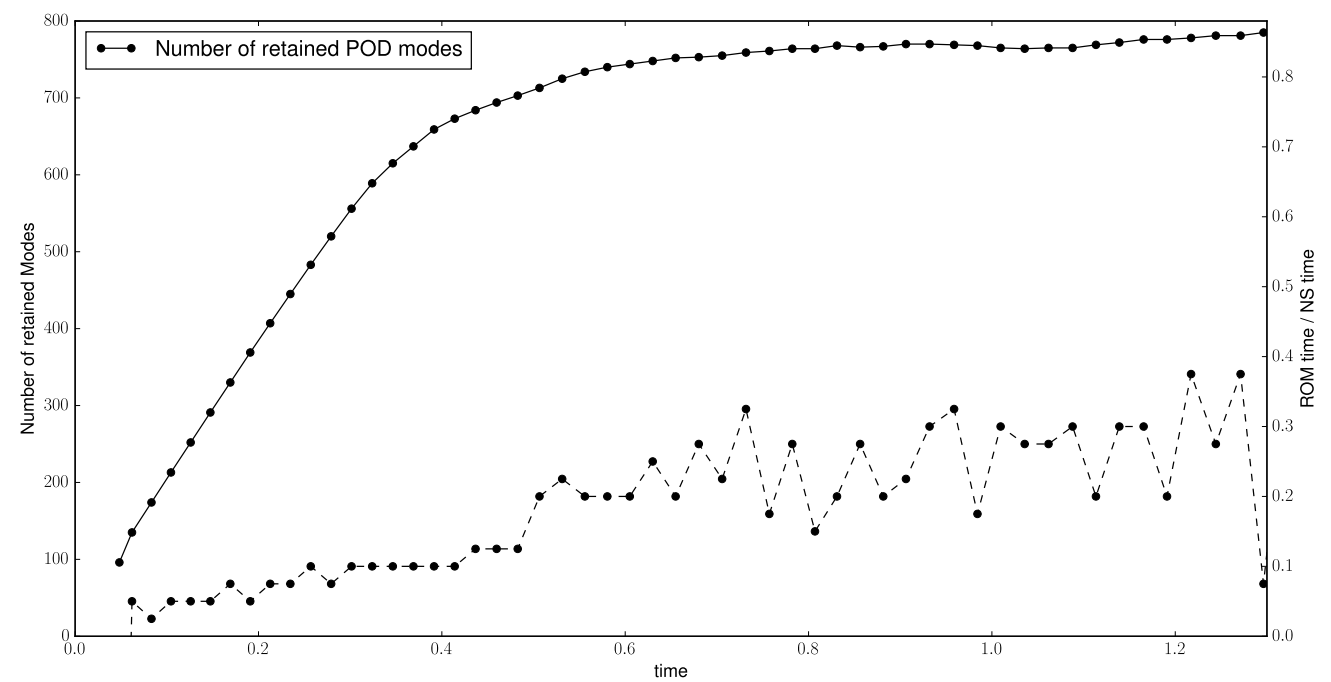

Figure 6.9: Number of retained modes and ROM vs NS times ratio for the $M=0.3$ at sea level, $a=0.01 \mathrm{rad}$ and $f=10.0 \mathrm{~Hz}$

The CPU acceleration factor, defined as

$$
\text { CPU acceleration }=\frac{\text { NS CPU time }}{\text { ROM CPU time }},
$$

is, however, relatively small, and the performance of the ROM is significantly worse 
than in the inviscid case.

If the amplitude of the oscillation is increased by an order of magnitude, to the case with $a=0.1 \mathrm{rad}$, the resultant time history can be seen in figure 6.10. The CPU acceleration factor for this case (see figure 6.11) is approximately two times higher than for the $a=0.01$ case, despite being a more demanding case, as highlighted in the inviscid case.

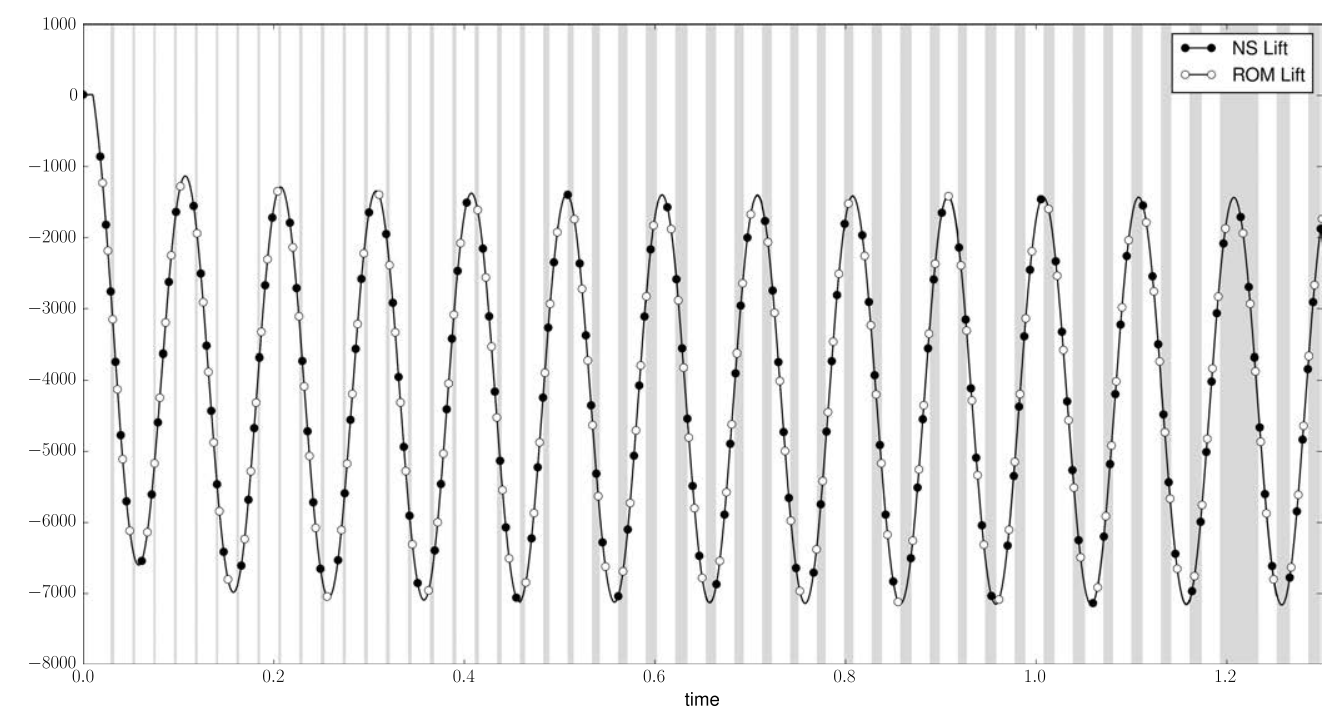

Figure 6.10: Counterpart of figure 6.8 for $a=0.1 \mathrm{rad}$

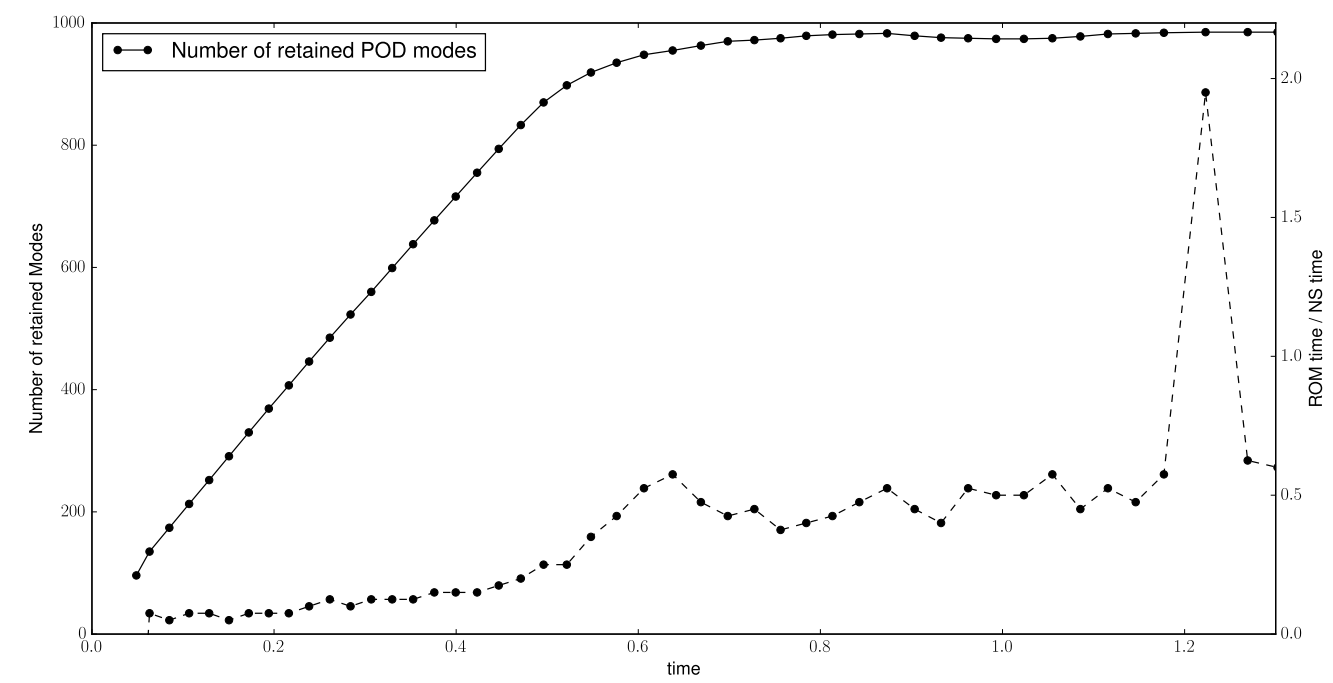

Figure 6.11: Number of retained modes and ROM vs NS times ratio for the $\mathrm{M}=0.3$ at sea level, $a=0.1 \mathrm{rad}$ and $f=10.0 \mathrm{~Hz}$

These results confirm the expected influence of the full solver numerical noise on the $\mathrm{ROM}$ efficiency. In other words, the higher the excitation, the higher the correlation between the primitive variables values in the flow field and the body motion, and hence the lower the noise effect.

Despite this deficiency, the ROM strategy has still shown to be able to update itself 
adequately in such a way that the efficiency steadily increases. Even when the CPU acceleration factor of the ROM might be considered small, it needs to be mentioned that due to the slow convergence of the full numerical solver, the time spent by each ROM update plus the iterations is negligible compared to a single full solver iteration.

\subsubsection{Results for the time dependent transonic flow at $M=0.8$}

Let us now increase the Mach number to $M=0.8$, maintaining the sea level altitude to facilitate the comparison with the subsonic case. For this transonic flow, similarly to the NACA0010 airfoil used in the inviscid case, the NACA0012 airfoil exhibits strong shocks in the pressure and suction sides, even at zero angle of attack. The same comments relative to the shock motion as in the inviscid case are also applicable here, with the particular addition of small regions in the vicinity of the shock where the viscous effects are important and need to be captured.

The solution has been performed equivalently to the subsonic case presented above, with the exception of a smaller $\Delta T^{\mathrm{CFD}}$, since the ROM needs to be updated more frequently to move along not too different shock positions, based on the lessons learned during the inviscid simulations. The tunable parameters of the problem appearing in $\S 2.3 .2$ are selected for the transonic case at $\mathrm{M}=0.8$ as

$$
T_{0}^{\mathrm{CFD}}=0.05 \mathrm{~s}, \quad \Delta T^{\mathrm{CFD}}=0.005 \mathrm{~s}, \quad \epsilon_{1}=10^{-10}, \quad K=20 .
$$

As in the former subsection, we concentrate in pitch motions, which are more demanding for the POD on the fly ROM. For the pitch oscillations at the lowest forcing amplitude in equation (6.23) and the low forcing frequency, $f=2.5 \mathrm{~Hz}$, which in the present case gives a reduced frequency $k=0.029$, the outcome of the POD on the fly method is given in figure 6.12. The length of the time intervals during which the ROM is used slowly increases along the simulation, although with an important oscillation, as it can also be seen in figure 6.13. The forces produced on the airfoil with both methods are plot indistinguishable.

A similar, but slightly better, result can be seen if the frequency is increased to $f=10.0 \mathrm{~Hz}$ (reduced frequency $k=0.12$ ) in figure 6.14 and figure 6.15.

\subsubsection{Remarks on the ROM efficiency}

In the previous sections the selection of parameters and solution method used has been highlighted. Let's turn now the attention to the effect of different solution methods and parameters on the ROM efficiency. For this, the efficiency of the ROM for different values and solution strategies will be evaluated for several relevant conditions presented above. 


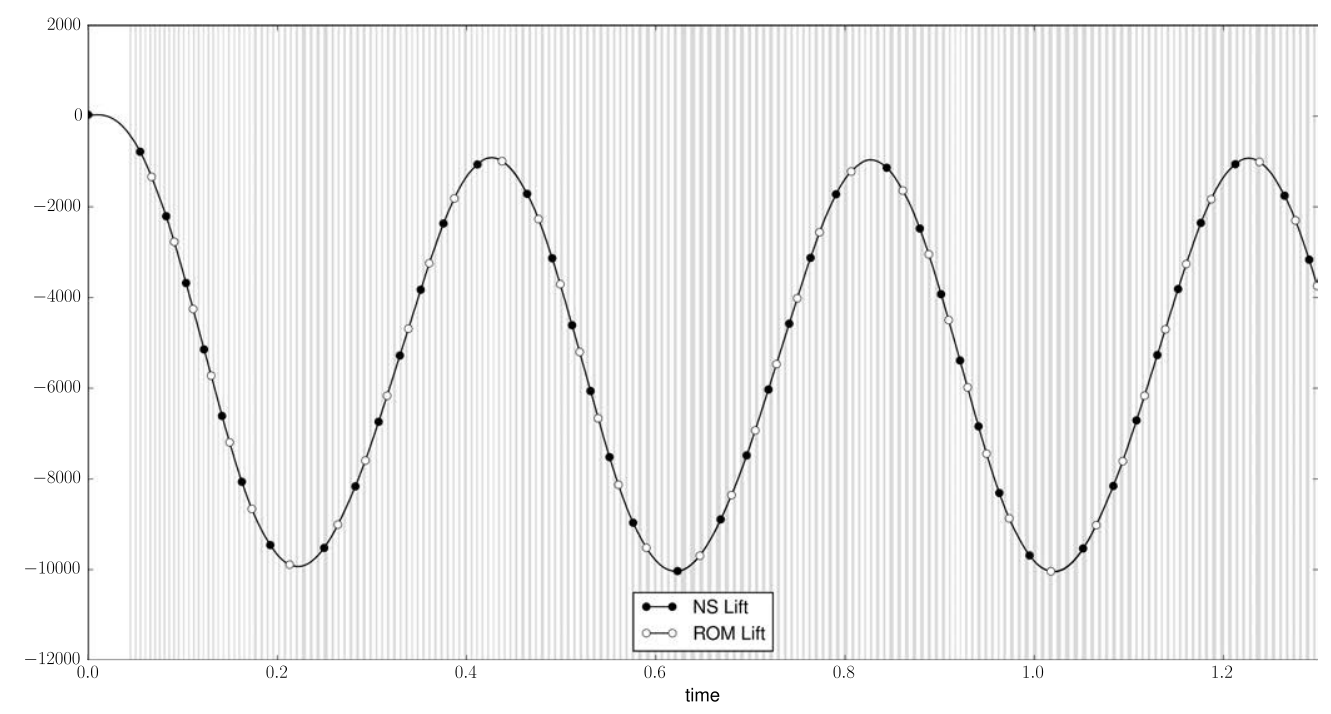

Figure 6.12: POD on the fly vs. the CFD solver, with $\mathrm{M}=0.8$ at sea level, considering pitch motion with $a=0.01 \mathrm{rad}$ and $f=2.5 \mathrm{~Hz}$. Those intervals in which the low dimensional ROM is used in the POD on the fly method indicated with shaded regions

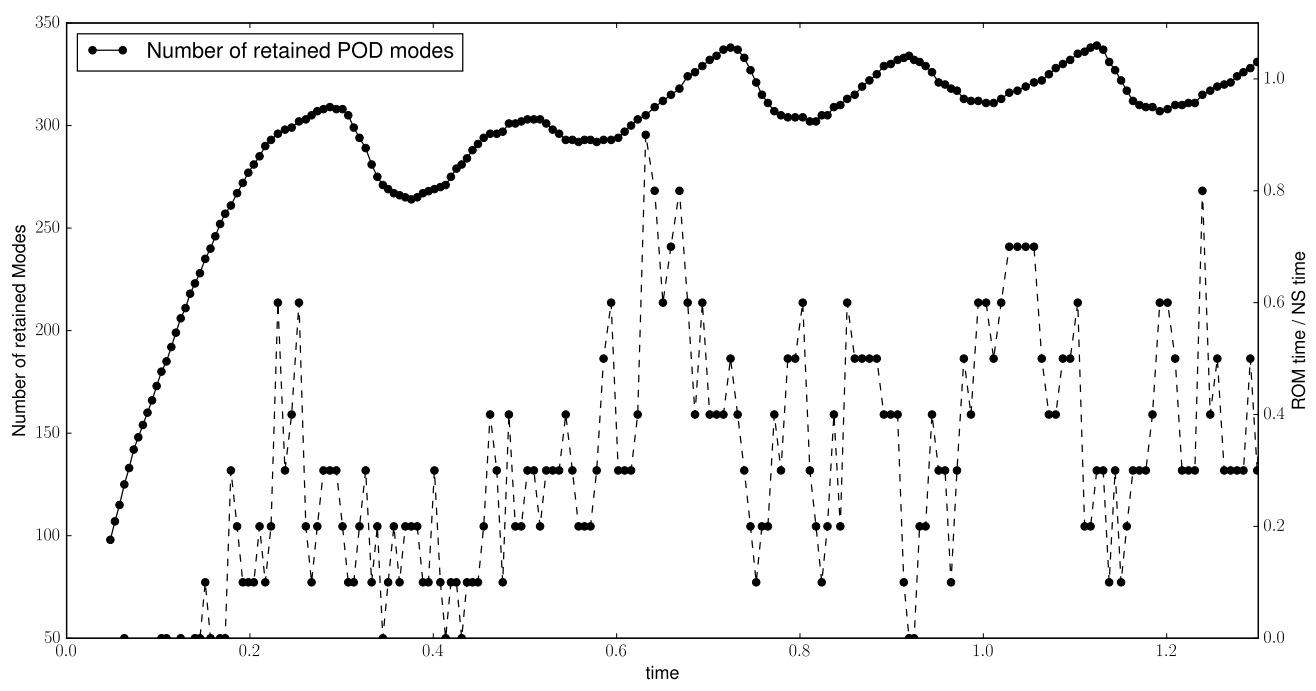

Figure 6.13: Number of retained modes and ROM vs NS times ratio for the $\mathrm{M}=0.8$ at sea level, $a=0.01 \mathrm{rad}$ and $f=2.5 \mathrm{~Hz}$ 


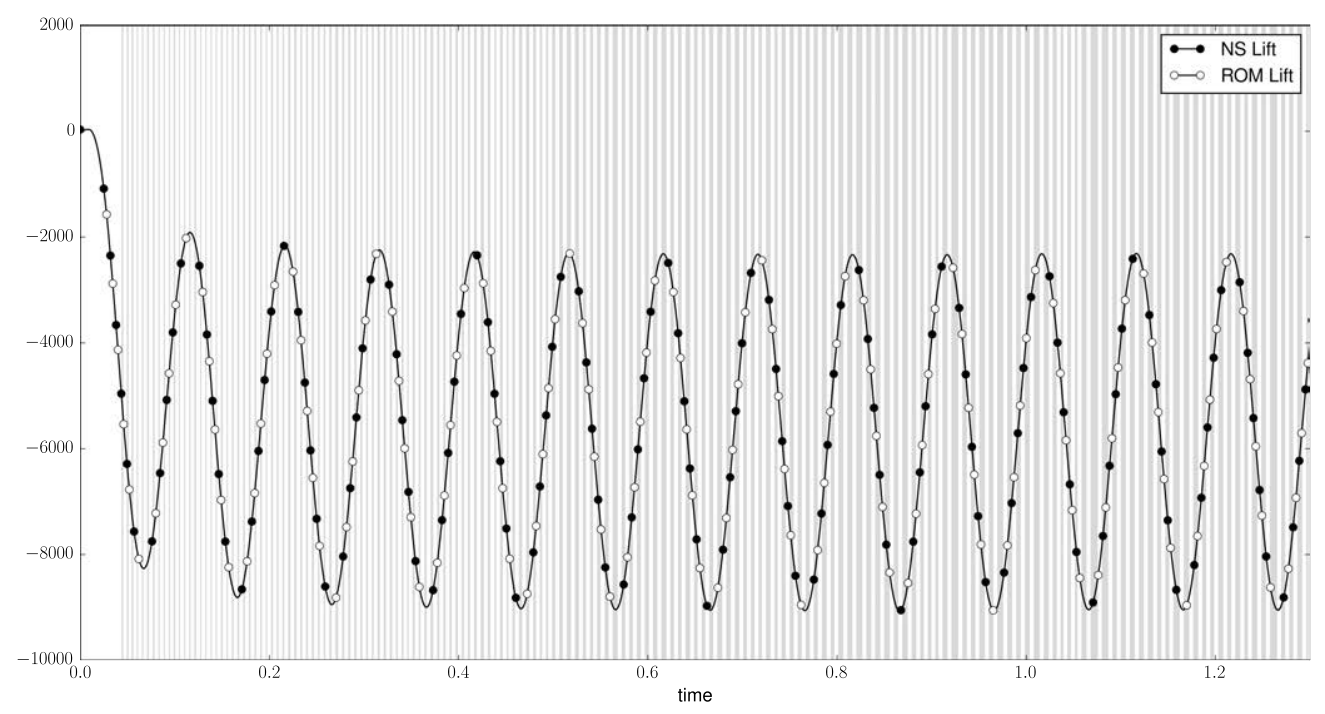

Figure 6.14: $\mathrm{POD}$ on the fly vs. the CFD solver, with $\mathrm{M}=0.8$ at sea level, considering pitch motion with $a=0.01 \mathrm{rad}$ and $f=10.0 \mathrm{~Hz}$. Those intervals in which the low dimensional ROM is used in the POD on the fly method indicated with shaded regions

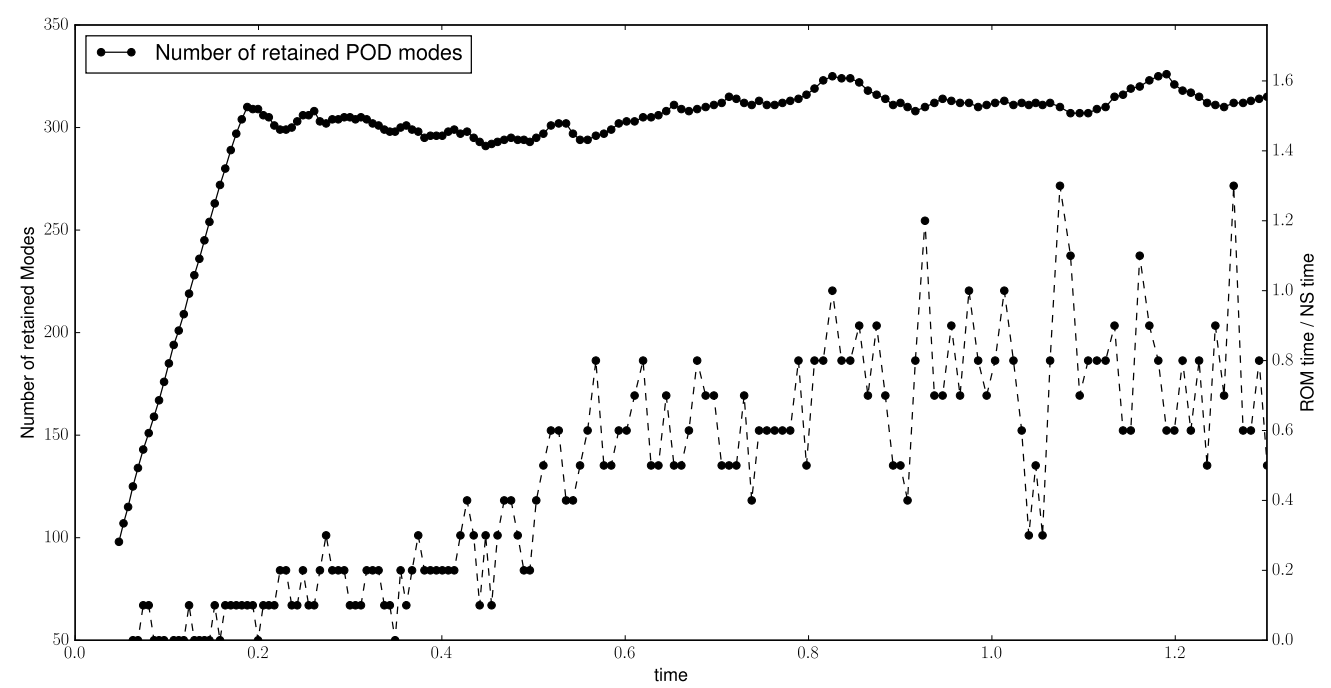

Figure 6.15: Number of retained modes and ROM vs NS times ratio for the $M=0.8$ at sea level, $a=0.01 \mathrm{rad}$ and $f=10.0 \mathrm{~Hz}$ 


\section{Turbulence POD strategy}

As mentioned before, all the calculations have been performed with $\tilde{\nu}$ as slave to the primitive variables, without any reference $\mathcal{Q}_{0}$. Figures 6.16-6.17 show the difference between the baseline strategy and the one with an independent POD basis presented above.

The ROM performance is very similar in both cases, although the baseline has the obvious advantage of reducing the need to solve the turbulence auxiliary variable transport equations.

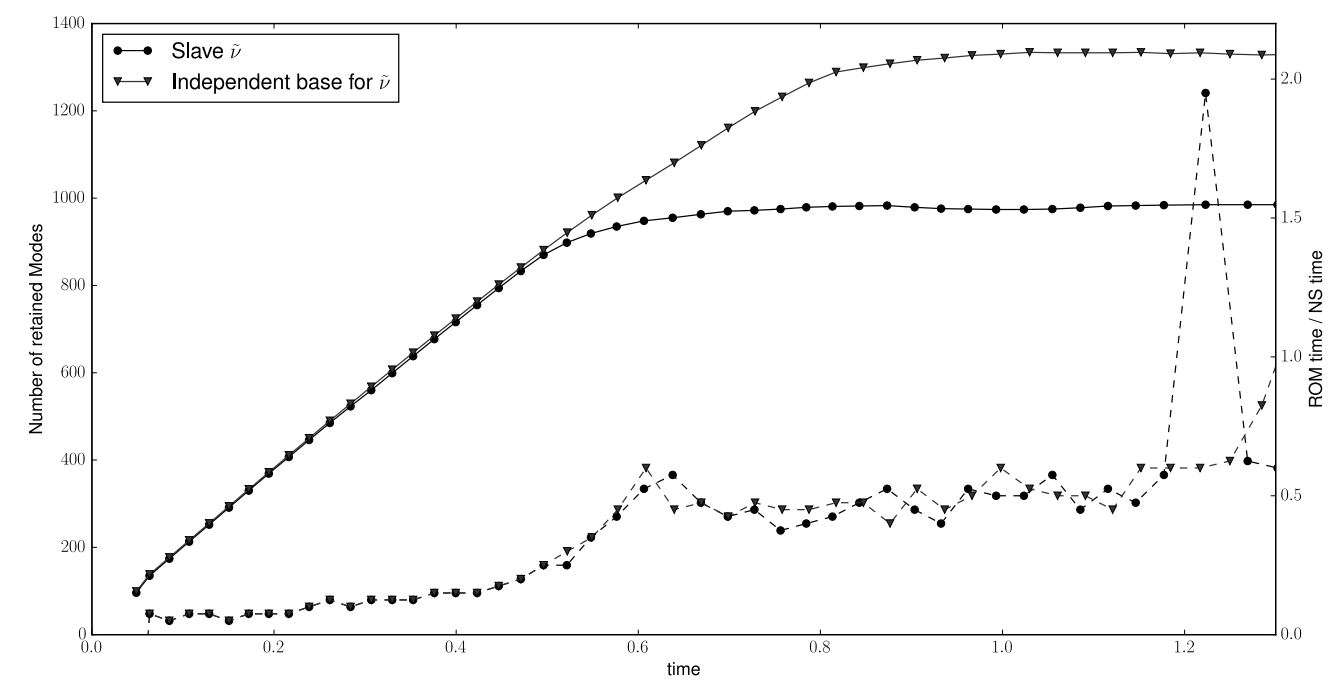

Figure 6.16: Turbulence solution strategy effect on the number of retained modes $(-)$ and $\mathrm{ROM}$ vs NS times ratio (--) for the $\mathrm{M}=0.3, \mathrm{a}=0.1 \mathrm{rad}$ and $\mathrm{f}=10 \mathrm{~Hz}$ case

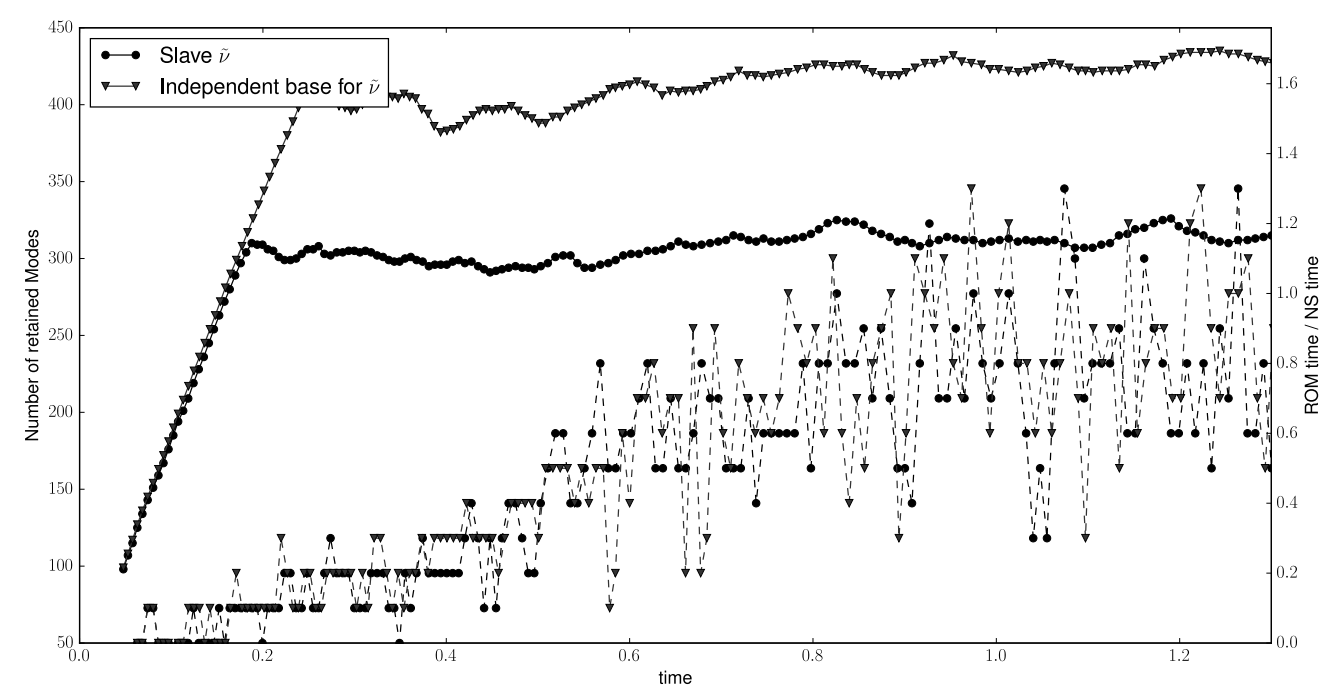

Figure 6.17: Turbulence solution strategy effect on the number of retained modes $(-)$ and $\mathrm{ROM}$ vs NS times ratio (- - ) for the $\mathrm{M}=0.8, \mathrm{a}=0.01 \mathrm{rad}$ and $\mathrm{f}=10 \mathrm{~Hz}$ case 


\section{Effect of $\Delta T^{\mathrm{CFD}}$}

Figure 6.18 shows the effect of $\Delta T^{\mathrm{CFD}}$ on the ROM performance. As shown in the inviscid case, the ROM performance for the initial steps seems to be better the lower $\Delta T^{\mathrm{CFD}}$. As the solution time evolves the contrary effect can be seen.

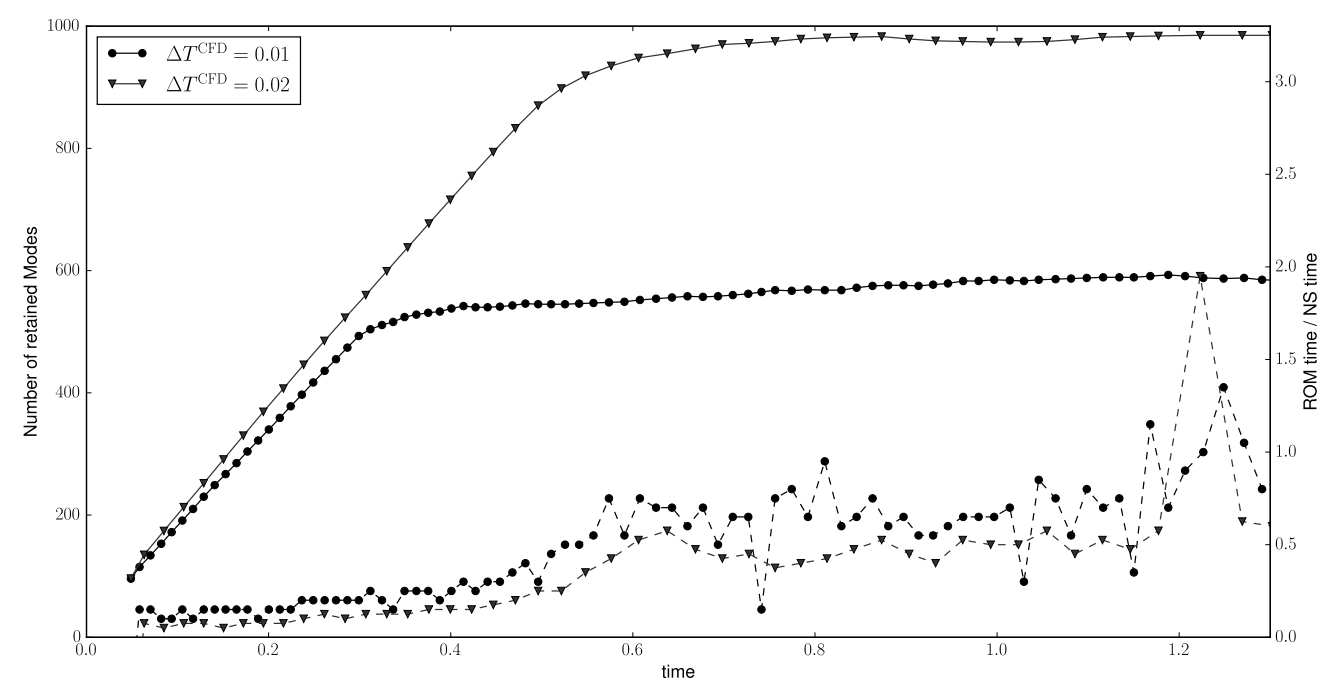

Figure 6.18: $\Delta T^{\mathrm{CFD}}$ effect on the number of retained modes $(-)$ and ROM vs NS times ratio (- -) for the $\mathrm{M}=0.3, \mathrm{a}=0.1 \mathrm{rad}$ and $\mathrm{f}=10 \mathrm{~Hz}$ case

\section{Effect of $\varepsilon_{1}$}

In a similar way as for the inviscid case, the effect of reducing the value of $\varepsilon_{1}$ on the ROM efficiency has been studied by, without modifying any parameter or solution strategy, running three simulations for the cases of $\varepsilon_{1}=10^{-8}, \varepsilon_{1}=10^{-10}$ (the nominal) and $\varepsilon_{1}=10^{-12}$. As it can be seen in figure 6.19 , the number of modes significantly increases, but the efficiency of the ROM remains equivalent.

\section{Convection differencing scheme}

During the CFD validation phase for the 2DZP case ( $\S D .1$ ), it was observed the need to use a second order convection differencing scheme for the momentum equations in order to accurately reproduce the viscous turbulent boundary layer, since too much artificial diffusion is present when using a first order differencing scheme. Let us now explore the effect of such change on the ROM efficiency.

Figure 6.20 shows the comparison for the three different approaches used in the 2DZP case. If the second order differencing scheme is only used for the momentum equation, the number of modes is reduced and the ROM efficiency increased. If only a first order differencing scheme is used for all the equations very few modes are extracted and the ROM efficiency is further improved. This points to the fact that the inclusion of the second order differencing scheme jeopardizes the overall convergence of the full numerical solver, increasing the amount of numerical noise present in the snapshots. 


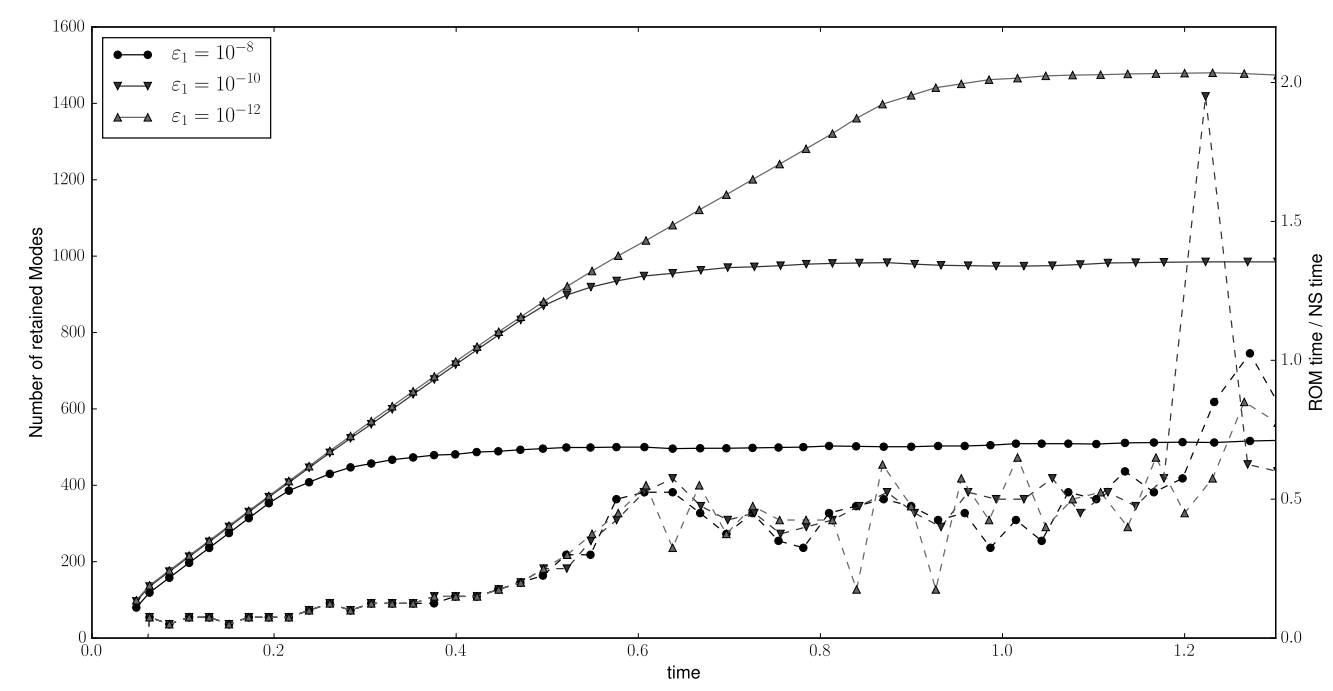

Figure 6.19: $\varepsilon_{1}$ effect on the number of retained modes $(-)$ and ROM vs NS times ratio (- - ) for the $\mathrm{M}=0.3, \mathrm{a}=0.1 \mathrm{rad}$ and $\mathrm{f}=10 \mathrm{~Hz}$ case

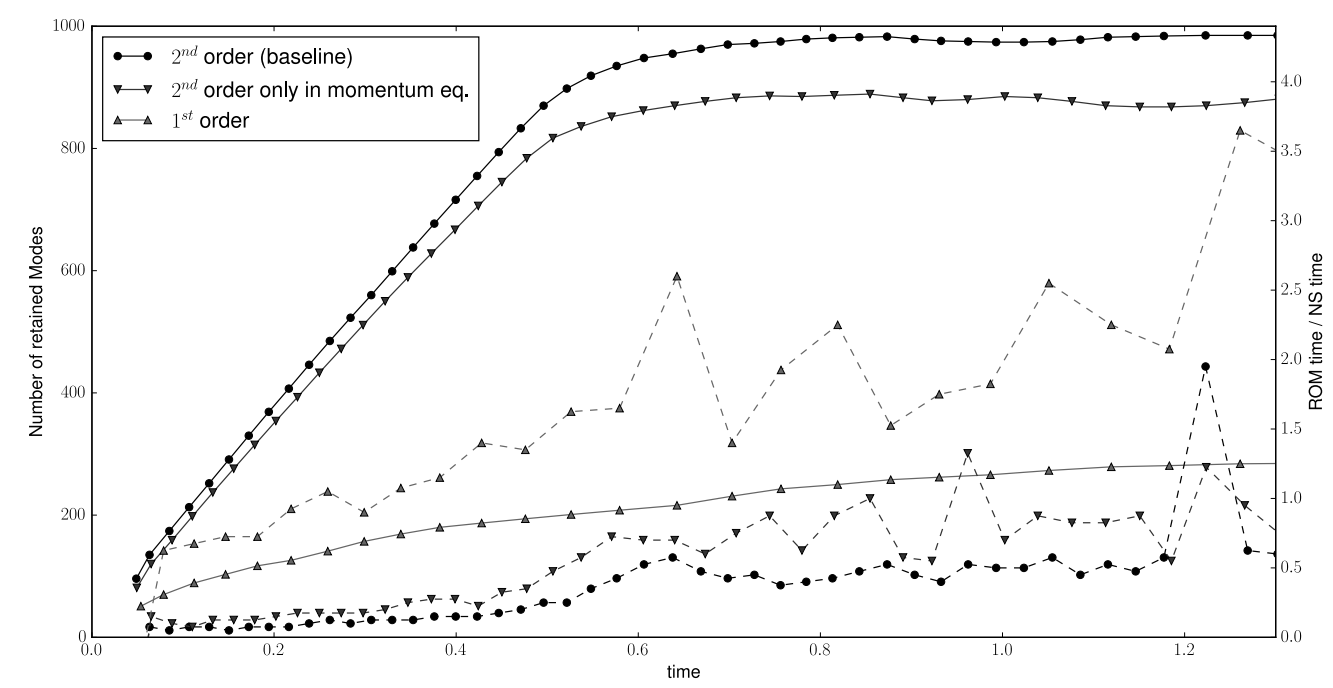

Figure 6.20: Convection differencing scheme effect on the number of retained modes $(-)$ and $\mathrm{ROM}$ vs NS times ratio (--) for the $\mathrm{M}=0.3, \mathrm{a}=0.1 \mathrm{rad}$ and $\mathrm{f}=10 \mathrm{~Hz}$ case 


\subsection{Aeroelastic simulation}

The same procedure as in the inviscid simulation has been carried out, coupling the turbulent viscous fluid solver and the self adaptive ROM to the structural model described in $\S 5.3$.

\subsubsection{Results for the subsonic flow at $M=0.3$}

As a first case to test the adaptive POD on the fly methodology for aeroelastic simulations using the viscous turbulent flow solver, we consider the impulse response of the structure in the subsonic regime, $\mathrm{M}=0.3$.

Similarly to the forced oscillation case, the turbulence auxiliary variable $\tilde{\nu}$ has been made slave to the primitive variables through the POD modes in the baseline approach. In the same way, the POD modes are calculated without any reference. The tunable parameters of the problem appearing in §2.3.2 are selected for the subsonic case considered here as

$$
T_{0}^{\mathrm{CFD}}=0.05 \mathrm{~s}, \quad \Delta T^{\mathrm{CFD}}=0.020 \mathrm{~s}, \quad \epsilon_{1}=10^{-10}, \quad K=20
$$

The aeroelastic simulations are run at a speed below (figure 6.21) and above (figure 6.23) the critical flutter speed. Overall the agreement is very good, with both simulation indiscernible from each other until the end of the simulation, point at which they divert slightly. Also, as in the forced oscillation case, the ROM is able to self adapt to increase its efficiency as more snapshots are available (see figures 6.22-6.24). However, due to the same performance issues as for the forced oscillation, the CPU acceleration factor is relatively small.

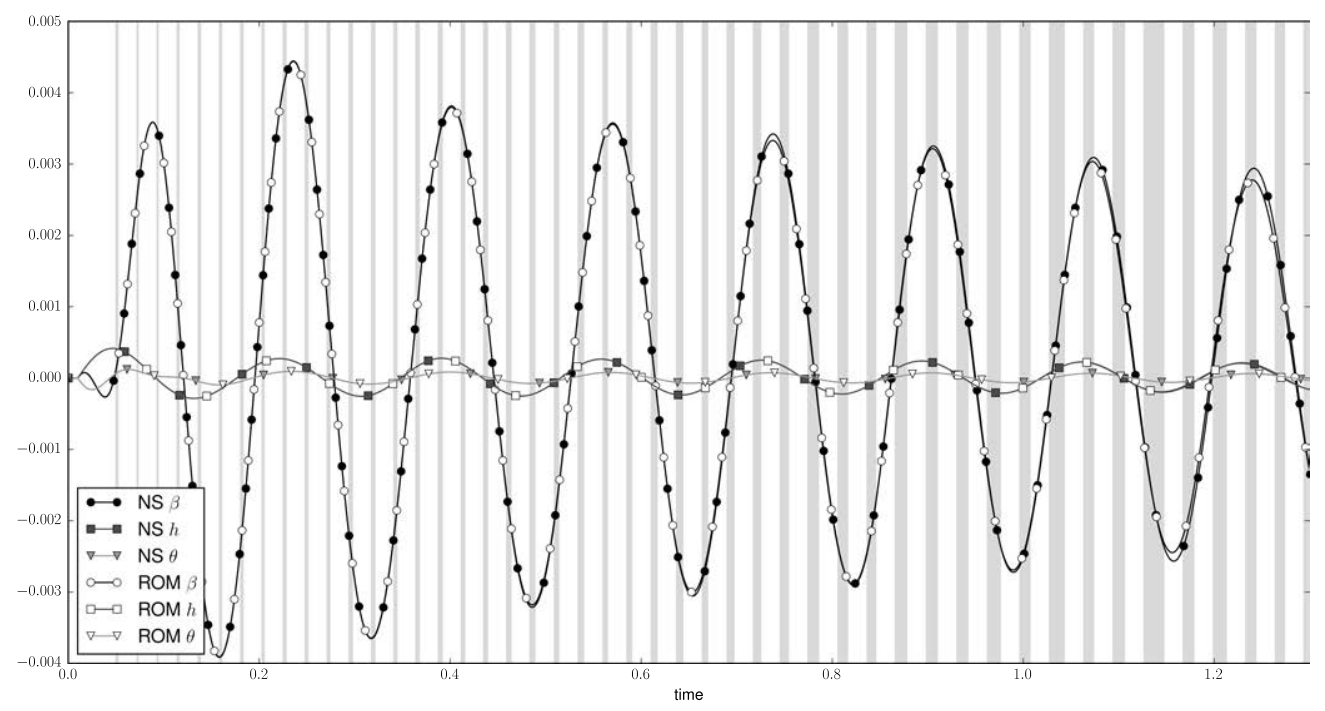

Figure 6.21: POD on the fly vs. the CFD solver. $M=0.3$ at a speed below flutter point 


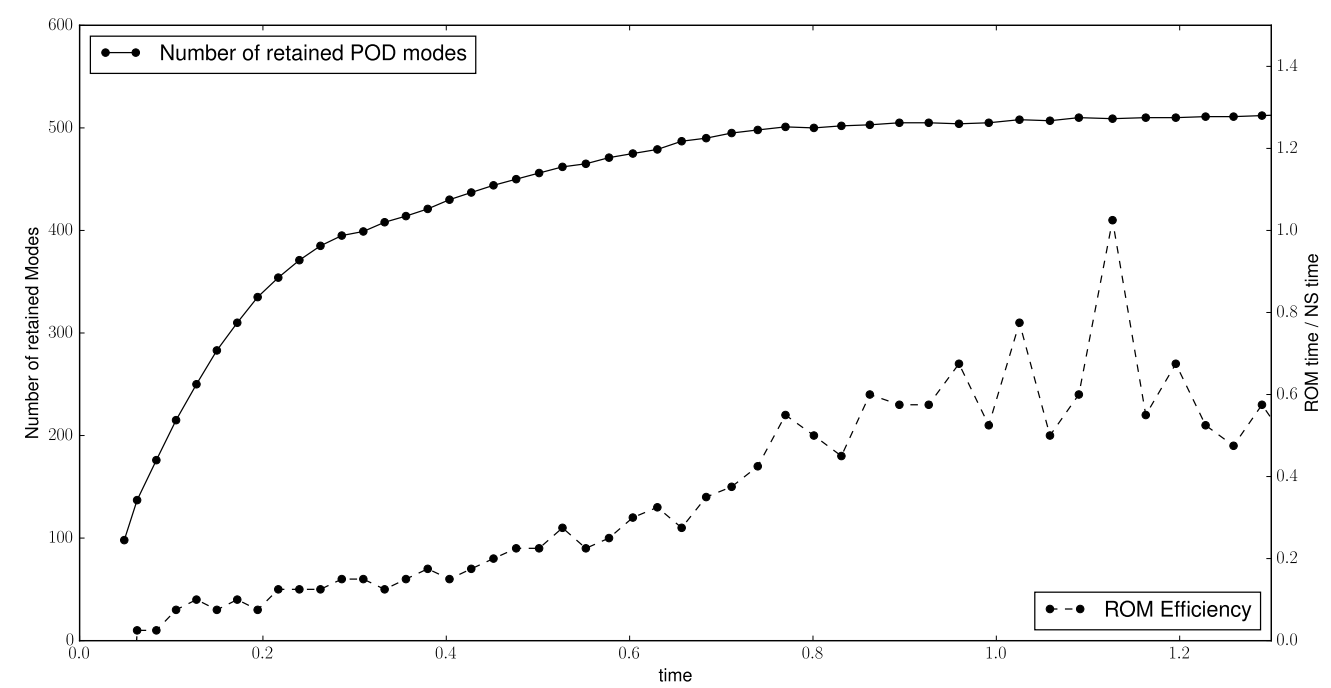

Figure 6.22: Number of retained modes and ROM vs NS times ratio for the $M=0.3$ at a speed below flutter point

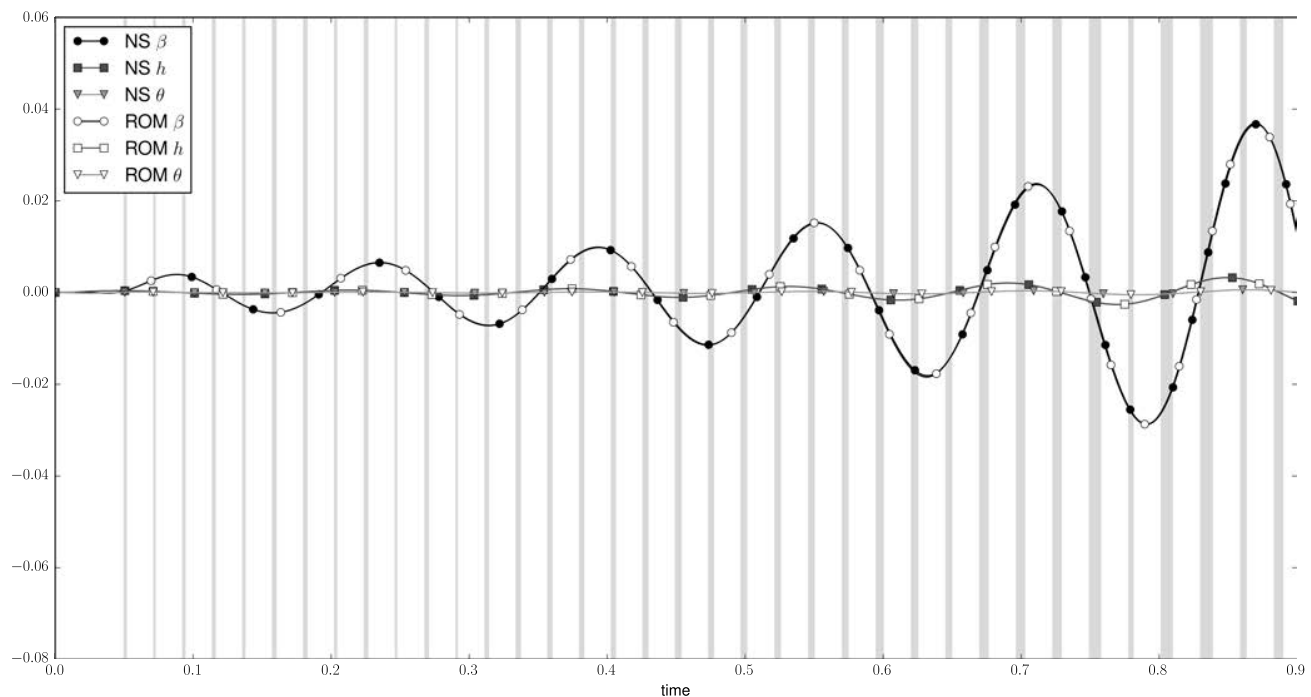

Figure 6.23: POD on the fly vs. the CFD solver. $\mathrm{M}=0.3$ at a speed above flutter point 


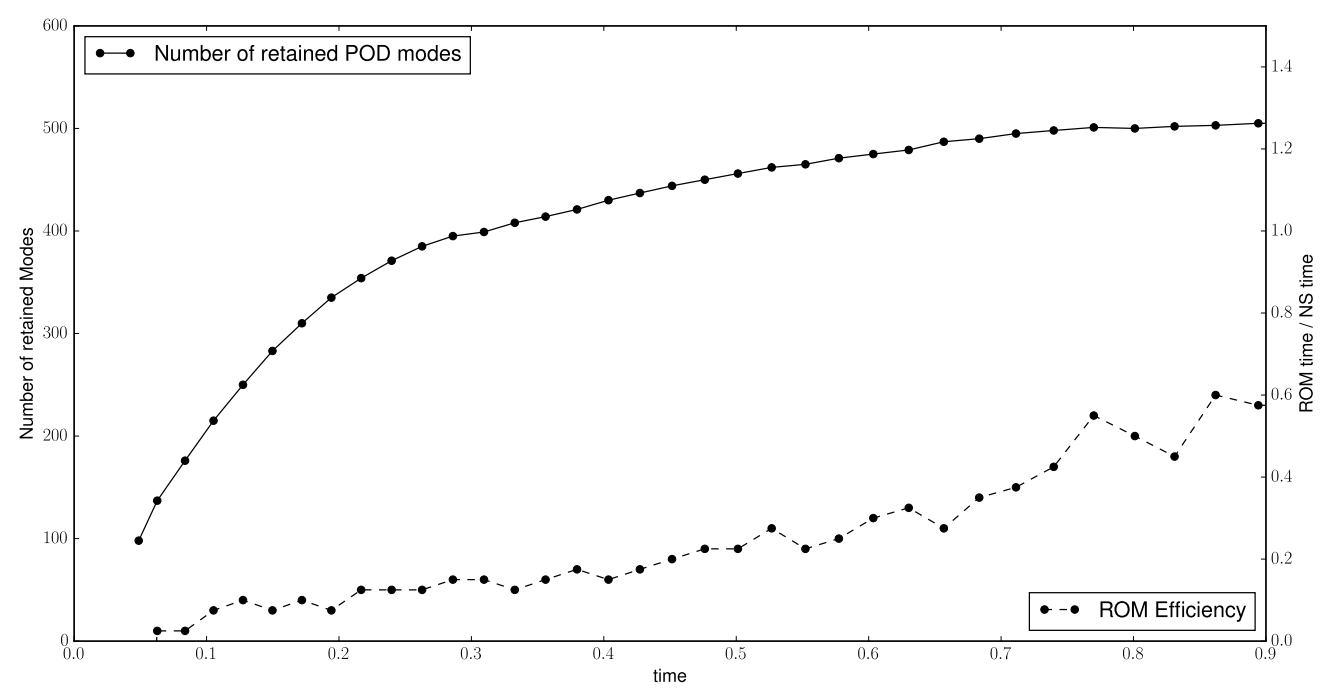

Figure 6.24: Number of retained modes and ROM vs NS times ratio for the $M=0.3$ at a speed above flutter point

\subsubsection{Results for the transonic flow at $M=0.8$}

Let us now increase the Mach number to $M=0.8$. The same comments regarding the need to capture the shock movement as in the forced oscillation case apply here. The tunable parameters of the problem appearing in $\S 2.3 .2$ are selected for the transonic case considered here as

$$
T_{0}^{\mathrm{CFD}}=0.05 \mathrm{~s}, \quad \Delta T^{\mathrm{CFD}}=0.005 \mathrm{~s}, \quad \epsilon_{1}=10^{-10}, \quad K=20
$$

The aeroelastic simulations are run at a speed below (figure 6.25) and above (figure 6.27) the critical flutter speed. Overall the agreement is excellent, better than in the subsonic case, with both simulation indiscernible from each other. Also, the ROM is able to self adapt to slowly increase its efficiency as more snapshots are available (see figures 6.28-6.26). The results are very similar to the forced oscillation case, where the ROM efficiency shows a significant oscillation. It must be noted that for the unstable case there are certain intervals for which the ROM is unable to continue the simulation.

\subsubsection{Remarks on the ROM efficiency}

In the previous sections the selection of parameters and solution method used has been highlighted. Let's turn now the attention to the effect of different parameters on the ROM efficiency. For this, the efficiency of the ROM for different parameter values will be evaluated for some relevant conditions presented above.

\section{Effect of $\Delta T^{\mathrm{CFD}}$}

Figure 6.29 shows the effect of $\Delta T^{\mathrm{CFD}}$ on the ROM performance. As shown in the inviscid case and the forced oscillation case, the ROM performance for the initial steps 


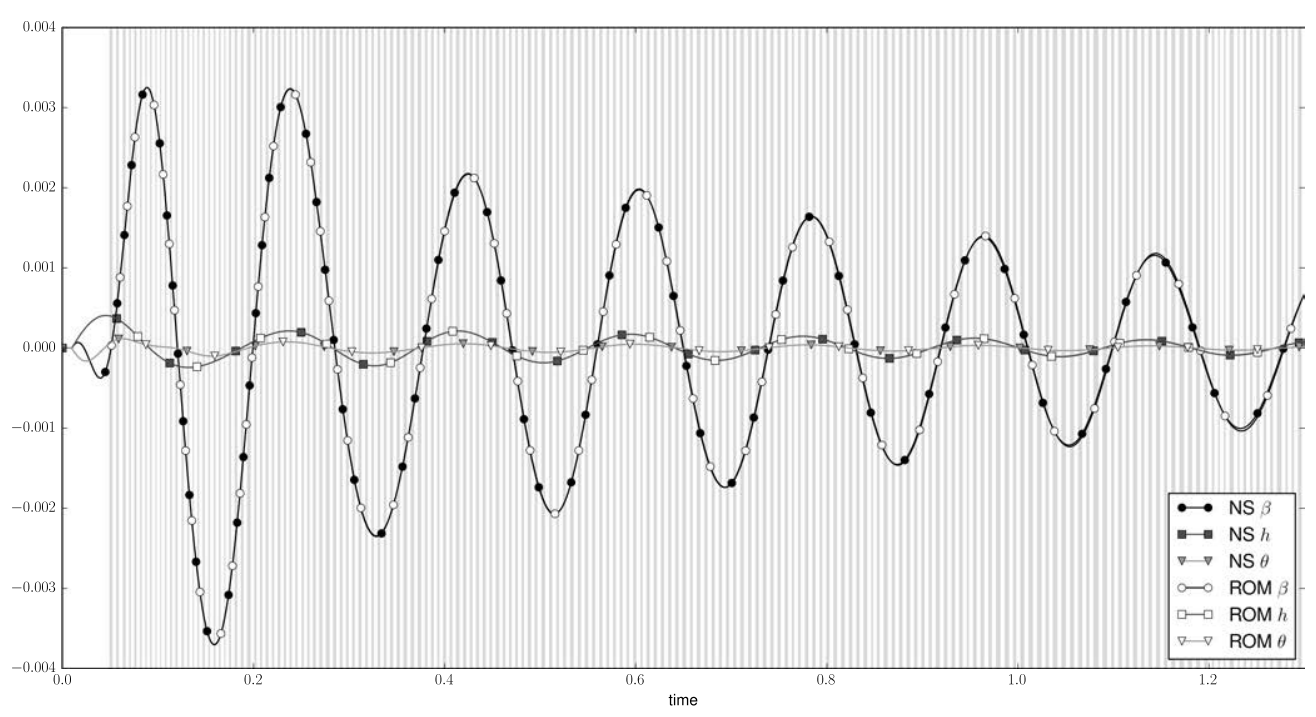

Figure 6.25: POD on the fly vs. the CFD solver. $M=0.8$ at a speed below flutter point

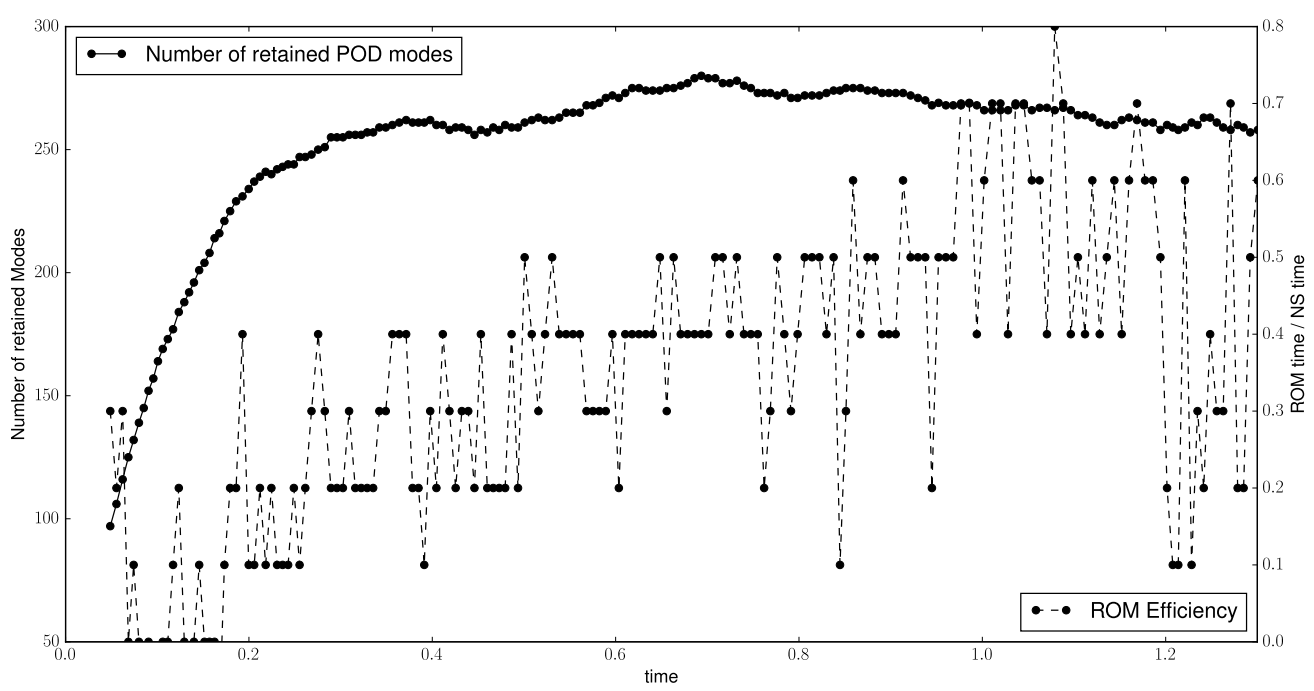

Figure 6.26: Number of retained modes and ROM vs NS times ratio for the $M=0.8$ at a speed below flutter point 


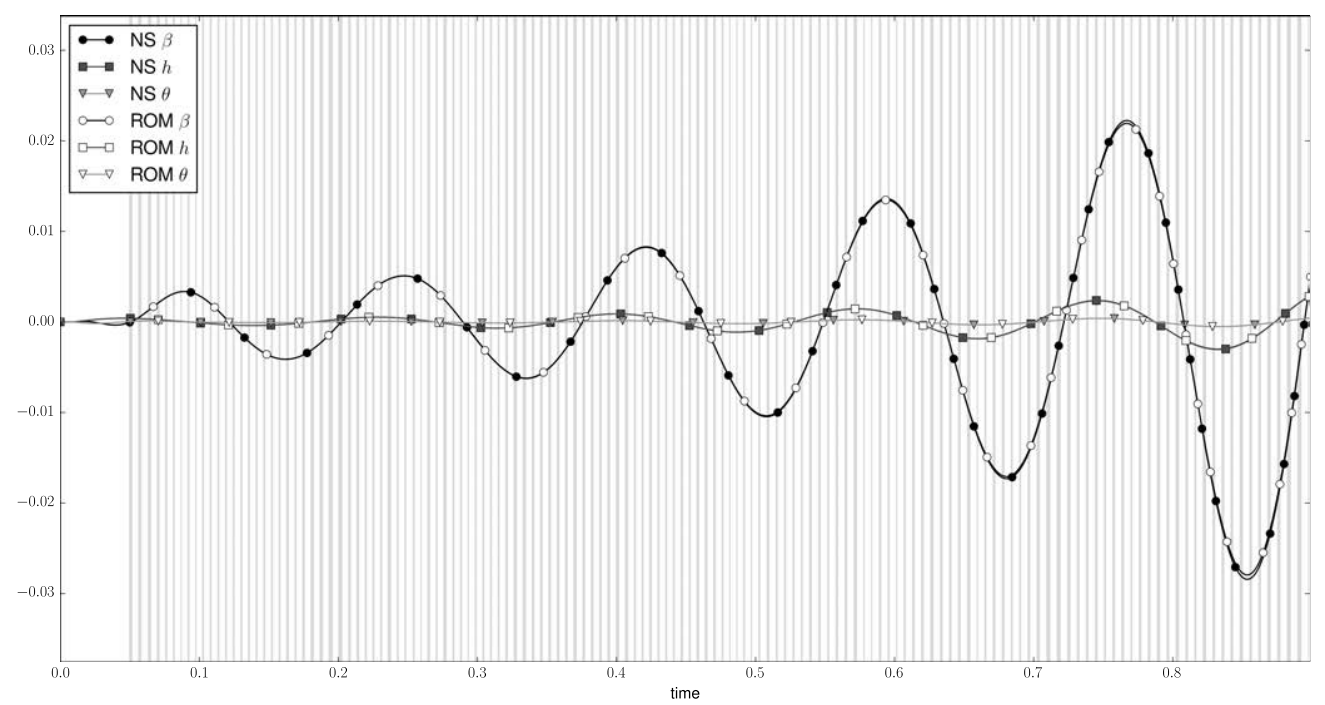

Figure 6.27: POD on the fly vs. the CFD solver. $M=0.8$ at a speed above flutter point

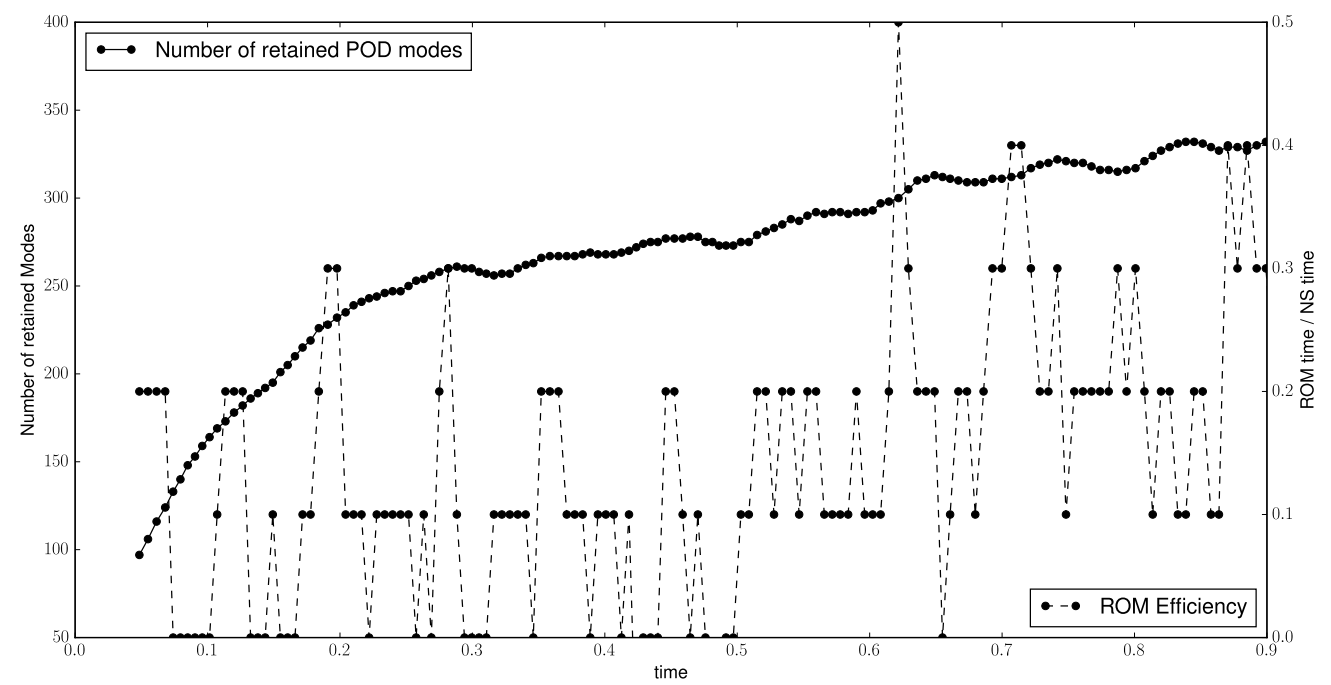

Figure 6.28: Number of retained modes and ROM vs NS times ratio for the $M=0.8$ at a speed above flutter point 
seems to be better the lower $\Delta T^{\mathrm{CFD}}$. Both simulations are very close as the solution time evolves. Also relevant is the fact that the higher value of $\Delta T^{\mathrm{CFD}}$ promotes the use of a higher number of POD modes in the ROM.

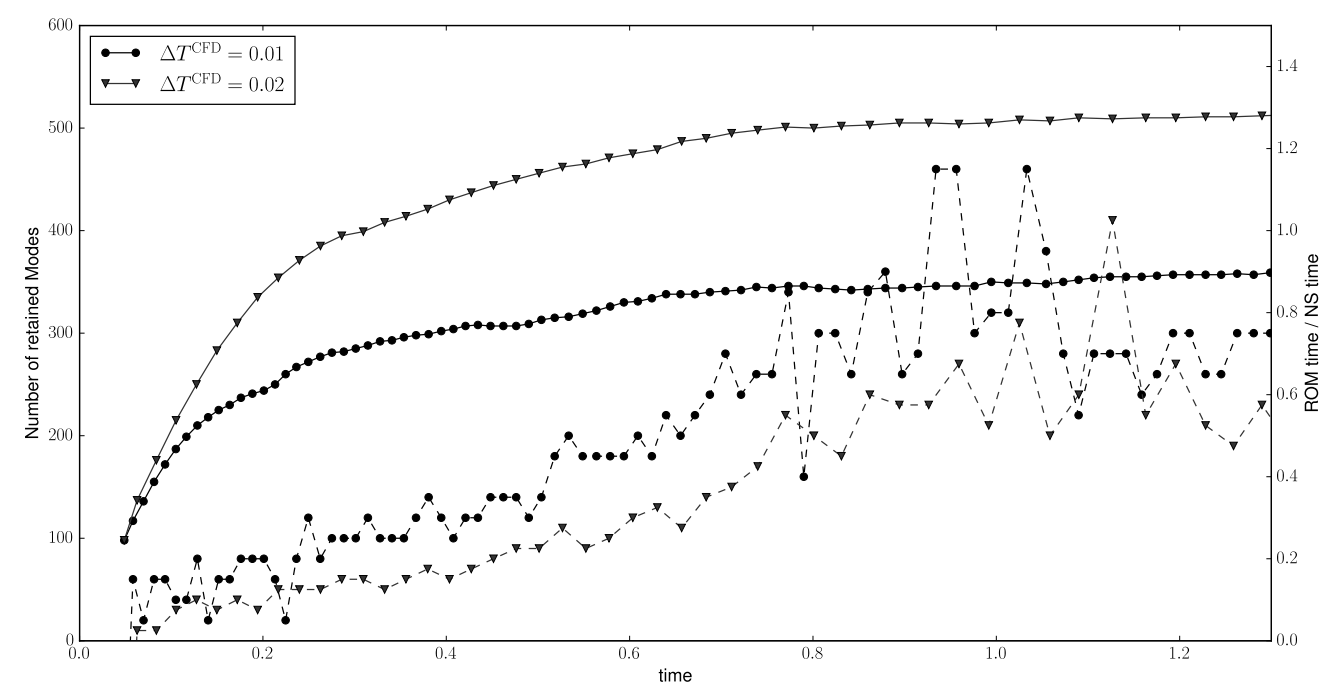

Figure 6.29: $\Delta T^{\mathrm{CFD}}$ effect on the number of retained modes (-) and ROM vs NS times ratio (- -) for the $\mathrm{M}=0.3$ case at a speed below the flutter point

\section{Effect of $\varepsilon_{1}$}

The effect of reducing the value of $\varepsilon_{1}$, the parameter that controls the relative RMS error of the POD modes truncation, has been studied by, without modifying any parameter or solution strategy, running three simulations for the cases of $\varepsilon_{1}=10^{-8}, \varepsilon_{1}=10^{-10}$ (the nominal) and $\varepsilon_{1}=10^{-12}$. As it can be seen in figure 6.30 , the number of modes significantly increases, but the efficiency of the ROM remains equivalent. This effect is consistent with the observations for the forced oscillation case.

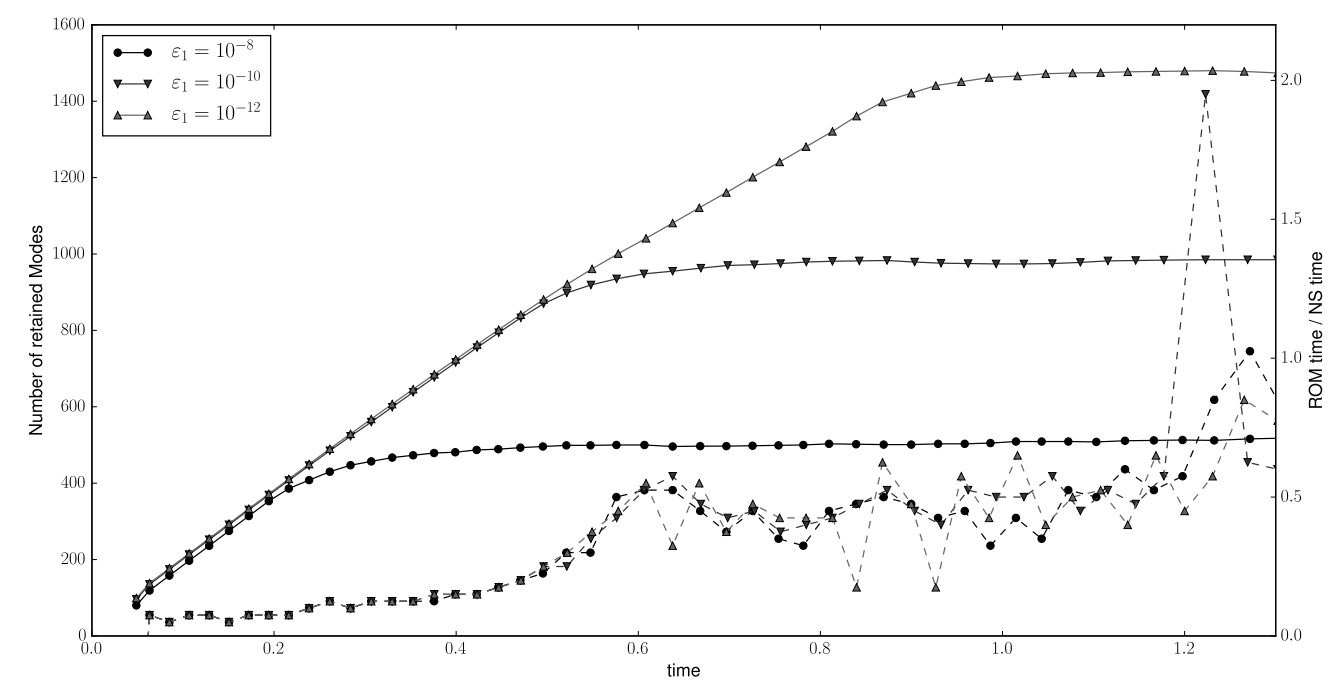

Figure 6.30: $\varepsilon_{1}$ effect on the number of retained modes $(-)$ and ROM vs NS times ratio (- -) for the $M=0.3$ case at a speed below the flutter point 


\subsection{Conclusions}

The extension of the novel POD on the fly method to the simulation of unsteady aerodynamics, and its application to aeroelastic simulations, has been successfully demonstrated for viscous turbulent conditions. Several new ingredients have been incorporated additionally to the method, like the ability to evolve the turbulence auxiliary variable in parallel to the primitive variables.

In order to demonstrate the application of the method, a two-dimensional airfoil has been considered, in contrast with the more computationally expensive, and expected to have higher gains, three-dimensional lifting surface.

The method has shown good accelerations for the subsonic case both in the case of forced motions (pitching and heave oscillations) and impulse response. In the transonic case, the method has to deal with the motion of the strong shocks present on the airfoil, which imposes a shorter validity of the ROM, but the ROM is still capable of performance increases. Overall, it produces results without any loss of accuracy, when compared with the full numerical solver. However, the resulting CPU acceleration factors are smaller than in the inviscid case. This can be attributed to the fact that the numerical solver is slower to converge and, additionally, it requires schemes (to reduce the amount of artificial dissipation) that introduce higher relative levels of numerical noise.

Nevertheless, it has been shown to have a high potential to speed up viscous turbulent unsteady aerodynamic calculations in the framework of computational dynamic aeroelasticity. 


\section{Chapter 7}

\section{Conclusions and future work}

A novel approach has been developed on the creation of a reduced order model ( $R O M$ ) for unsteady aerodynamics, keeping in mind, as final target, its ability to perform computational aeroelastic simulations. The method is based on the well established POD on the fly methodology, which has been successfully applied in a variety of problems (e.g., the Complex Ginzburg-Landau equation) that exhibit fairly complex dynamics (Terragni and Vega, 2014), but are far less demanding from the reduced order modeling point of view than unsteady aerodynamics.

The method has been described in detail in chapter 2. It consists of a self updating ROM based on the modes obtained from the proper orthogonal decomposition (POD) of a previous set of snapshots, or a combination thereof. The ROM is made self adaptive thanks to running in interspersed intervals with the full numerical solver. After the full solver is run for a predetermined amount of time, the POD modes are calculated, and the ROM is constructed by projecting the fluid governing equations into such modes. The residuals are minimized and the equations evolved in time until the ROM loses its validity, due to changes in the system dynamics or truncation instabilities. This event is captured, in the present work, by the inability of the ROM to reduce the residual beyond a fixed, and tunable, factor. At that point, the simulation is reverted to the full solver for a given amount of time, the POD modes calculated and combined with the previous set of modes to form the new ROM, which is used to continue evolving the fluid state. This process repeats until the end of the simulation.

Since the method relies in having full understanding and control over the full numerical solver, a new computational fluid dynamics (CFD) solver of the unsteady Reynolds averaged Navier-Stokes equations (URANS) has been developed and validated for the work in this thesis. A detailed description has been given in chapter 3. It is based on the central-upwind scheme method of Kurganov, Noelle and Petrova (Kurganov et al., 2001), with additions from Greenshields (Greenshields et al., 2010) and Darwish (Darwish and Moukalled, 2003), amongst others. It is additionally coupled with the Spalart-Allmaras turbulence model (Allmaras and Johnson, 2012). The code has been validated for inviscid and turbulent viscous simulations.

In the framework of computational aeroelasticity, chapter 4 has shown how the 
CFD solver has been coupled to a structural solver, such that the fluid forces acting on the body affect its motion and deformation. The computational mesh is subsequently deformed, following the work by Luke (Luke et al., 2012), such that it remains conformal to the structure. This coupling is performed in a loose way to perform computational aeroelasticity simulations.

The method has been applied to the inviscid compressible problem in chapter 5 , considering first the unsteady flow around a rigidly moving two dimensional airfoil, in both subsonic and transonic conditions, covering both pitch and heave motions and various values of the forcing amplitude and frequency. Next, it has been applied to simulate the coupled aeroelastic impulse response of the airfoil under the same compressible subsonic and transonic conditions for velocities above and below the critical flutter speed (the flutter stability boundary). The lack of viscosity poses an additional challenge, while still maintaining the basic ingredients of the fluid governing equations, hence it is considered a good benchmark to show the potential application of the method for aeroelastic simulations. For both the forced and the aeroelastic simulations, the results have shown a very good accuracy (plot indistinguishable results) and significant CPU acceleration factors as defined in equation (5.7), up to the point that in some simulations no more iterations with the full solver are necessary after a given time. Transonic simulations pose the additional challenge of having to deal with the shock motion, since such strong discontinuities can not be expressed as a linear superposition of the different modes. The ROM successfully solves this challenge using more frequent updates, so that it remains in regions where the shock motion is not significant. Overall, the results are very promising. In contrast to traditional preprocessed POD based ROMs, the present ROM has been shown to be capable of adapting to new dynamics that might emerge during the simulation.

The same set of simulations as for the inviscid case have been repeated, including viscosity and the addition of a turbulence model, in order to explore further adaptations required by the method for its application to viscous turbulent simulations. As such, the turbulence auxiliary variable evolution is slave to the primitive variables through the POD modes, since the turbulence transport equation is not projected onto the POD modes. As shown in chapter 6, the created ROM is able to evolve the fluid state in a similar way as for the inviscid cases, and the results produced are of high level of accuracy, but with smaller CPU acceleration factors. This can be attributed to the fact that the numerical solver is slower to converge and, additionally, it requires schemes that introduce a lower amount of artificial dissipation in order to reproduce turbulent conditions, thus introducing higher relative levels of numerical noise.

The developed method has been proven to be robust and efficient in reducing the computational cost associated with the calculation of unsteady aerodynamics, in the computational aeroelasticity framework. This has been demonstrated in this thesis with two-dimensional aeroelastic simulations. The extension to three-dimensional calculations is expected to provide higher CPU acceleration factors. This is due to the 
fact that the POD based ROM works by removing redundancies existing in the previously computed fluid domain snapshot matrix, which are usually higher for attached flows, relevant for industrial aeroelastic simulations. Additionally, the number of modes necessary to represent the solution do not grow as quickly as the number of numerical degrees of freedom when increasing the number of spatial dimensions. Three-dimensional numerical simulations are computationally more expensive and require a much larger number of cells, so it would require the CFD developed code to be parallelized in order to be able to cope with the higher computational cost and memory requirements.

One of the key ingredients of the method is the use of a subset of the cells for the POD modes calculations and projection of the governing equations. The selection of the subset has been performed ad-hoc in the examples above, in an attempt to find a robust one-fits-all approach for subsonic and transonic regimes. Several examples exist in the literature where the selection of the subset is performed based on optimal estimators, cells with maximum gradients, or even iterative approaches. For example, the addition of such methods would allow to select the subset based on the initial set of numerical solver runs (Rapún et al., 2017), so that the selection would be optimal for the condition run.

Turbulent viscous simulations, at least with the currently selected approach, have been shown to produce higher relative numerical noise, hence reducing the redundancy levels present in the snapshot matrix and jeopardizing the capacity of the ROM to further increase the overall computational efficiency. It is advised to explore other numerical schemes, and solution algorithms, that might reduce the relative noise levels, and truly show the full potential of the method, which so far has been demonstrated for the inviscid simulations performed in this thesis.

The POD on the fly method is specially suited for the creation of modes databases (Rapún et al., 2015) that can later be used for running with different conditions or mass cases. The inclusion of this enhancement in the method would significantly reduce the cost of FSI simulations even further, and enable its use in industrial environments.

Transonic static aeroelastic simulations, for which the time dependency is removed, are also a field where an increase in accuracy is required in the framework of maneuver simulations and performance estimations. One of the key difficulties of such simulations is the interaction between the fluid and the structure, alike in the applications performed in this thesis. It is expected that the method can be modified and adapted for such simulations with relative ease.

In conclusion, the POD on the fly methodology has been successfully extended to simulate unsteady flows with the main focus on computational dynamic aeroelasticity. The potential of the method has been shown by successfully running two-dimensional inviscid and viscous turbulent simulations. 


\section{Bibliography}

Ahrens, J. et al. (2005). "ParaView: An End-User Tool for Large-Data Visualization". The Visualization Handbook, p. 717.

Allmaras, S. R. and F. T. Johnson (2012). "Modifications and Clarifications for the Implementation of the Spalart-Allmaras Turbulence model". In: Seventh International Conference on Computational Fluid Dynamics (ICCFD7), pp. 1-11.

Alonso, D., A. Velazquez, and J. M. Vega (2009). "A Method to Generate Computationally Efficient Reduced Order Models". Computer Methods in Applied Mechanics and Engineering 198, pp. 2683-2691.

Alonso, D., J. M. Vega, and A. Velazquez (2010). "Reduced-Order Model for Viscous Aerodynamic Flow Past an Airfoil". AIAA Journal 48, pp. 1946-1958.

Astrid, P. et al. (2008). "Missing Point Estimation in Models Described by Proper Orthogonal Decomposition". IEEE Transactions on Automatic Control 53.10, pp. 2237-2251.

Balajewicz, M. J., E. H. Dowell, and B. R. Noack (2013). "Low-dimensional Modelling of High-Reynolds-Number Shear Flows Incorporating Constraints from the NavierStokes Equation". Journal of Fluid Mechanics 729, pp. 285-308.

Bardina, J et al. (1997). "Turbulence Modeling Validation". In: 28th Fluid dynamics conference, p. 2121.

Barone, M. F. et al. (2009). "Stable Galerkin Reduced Order Models for Linearized Compressible Flow". Journal of Computational Physics 228.6, pp. 1932-1946.

Bartels, R., C. Rumsey, and R. Biedron (2006). "CFL3D User's Manual. General Usage and Aeroelastic Analysis (Version 6.4)". NASA TM 214301, p. 2006.

Batina, J. T. (1985). Effects of Airfoil Shape, Thickness, Camber, and Angle of Sttack on Calculated Transonic Unsteady Airloads. Technical Report NASATM-86320. NASA.

Billah, K. and R. Scanlan (1991). "Resonance, Tacoma Narrows Bridge Failure, and Undergraduate Physics Textbooks". American Journal of Physics 59.2, pp. 118124.

Bisplinghoff, R. L., H. Ashley, and R. L. Halfman (1955). Aeroelasticity. AddisonWesley Publishing Company, Cambridge, Massachusetts.

Borm, O. (2010). "Transonic Density Based Flow Solver". In: 5th OpenFOAM conference, Sweden. 
Boussinesq, J. (1877). Essai sur la théorie des eaux courantes. Vol. 2. Imprimerie nationale, Paris.

Carlson, J.-R. (2011). Inflow/Outflow Boundary Conditions with Application to FUN3D. Technical Report NASA/TM-2011-217181. NASA.

Coles, D. and A. J. Wadcock (1979). "Flying-hot-wire Study of Flow Past a NACA 4412 Airfoil at Maximum Lift". AIAA Journal 17.4, pp. 321-329.

Collar, A. R. (1978). "The First Fifty Years of Aeroelasticity". Aerospace 2.5, pp. 12-20. Conner, M. et al. (1997). "Nonlinear Behavior of a Typical Airfoil Section with Control Surface Freeplay: a Numerical and Experimental study". Journal of Fluids and Structures 11.1, pp. 89-109.

Cook, P., M. Mcdonald, and M. Firmin (1979). Experimental Data Base for Computer Program Assessment. Advisory Report AR-138. AGARD.

Courant, R., K. Friedrichs, and H. Lewy (1967). "On the Partial Difference Equations of Mathematical Physics". IBM journal of Research and Development 11.2, pp. 215234.

Darwish, M. and F Moukalled (2003). "TVD Schemes for Unstructured Grids". International Journal of Heat and Mass Transfer 46.4, pp. 599-611.

Dowell, E., E. Crawley, et al. (1995). A Modern Course in Aeroelasticity. Kluwer Academic Publishers, Dodrecht.

Dowell, E., J. Thomas, and K. Hall (2001). "Transonic Limit Cycle Oscillation Analysis Using Reduced Order Aerodynamic Models". In: 42nd AIAA Structures, Structural Dynamics and Materials Conference and Exhibit, Seattle, WA.

Ellis, P. D. M. (2011). "Laser Palatoplasty for Snoring due to Palatal Flutter: a Further Report". Clinical Otolaryngology 19.4, pp. 350-351.

Garrick, I. E. (1933). Determination of the Theoretical Pressure Distribution for Twenty Airfoils. Technical Report 465. NACA.

Geuzaine, C. and J.-F. Remacle (2009). "Gmsh: A 3-D Finite Element Mesh Generator With Built-in Pre-and Post-processing Facilities". International Journal for Numerical Methods in Engineering 79.11, pp. 1309-1331.

Gordon, W. J. and C. A. Hall (1973). "Construction of Curvilinear Co-ordinate Systems and Applications to Mesh Generation". International Journal for Numerical Methods in Engineering 7.4, pp. 461-477.

Greenshields, C. J. et al. (2010). "Implementation of Semi-Discrete, Non-staggered Central Schemes in a Colocated, Polyhedral, Finite Volume Framework, for High-speed Viscous Flows". International Journal For Numerical Methods in Fluids 63.1, pp. 1-21.

Gregory, N. and C. O'reilly (1973). Low-Speed Aerodynamic Characteristics of NACA 0012 Aerofoil Section, Including the Effects of Upper-Surface Roughness Simulating Hoar Frost. HM Stationery Office, London.

Harten, A. (1983). "High Resolution Schemes for Hyperbolic Conservation Laws". Journal of Computational Physics 49.3, pp. 357-393. 
Hassig, H. J. (1971). "An Approximate True Damping Solution of the Flutter Equation by Determinant Iteration." Journal of Aircraft 8.11, pp. 885-889.

Heeg, J. et al. (2013). "Overview of the Aeroelastic Prediction Workshop". In: 51st AIAA Aerospace Sciences Meeting. Grapevine, TX, United States.

Hirsch, C. (2007). Numerical Computation of Internal and External Flows: The Fundamentals of Computational Fluid Dynamics. Butterworth-Heinemann, Oxford.

Holmes, P. (2005). "Ninety Plus Thirty Years of Nonlinear Dynamics: Less is More and More Different". International Journal of Bifurcation and Chaos 15.9, pp. 27032716.

Jasak, H (1996). "Error Analysis and Estimation for the Finite Volume Method with Applications to Fluid Flows, 1996". PhD thesis. University of London Imperial College.

Jasak, H., A. Jemcov, and Z. Tukovic (2007). "OpenFOAM: A C++ Library for Complex Physics Simulations". In: International Workshop on Coupled Methods in Numerical Dynamics. Vol. 1000, pp. 1-20.

Kalashnikova, I. et al. (2014). "Construction of Energy-Stable Projection-Based Reduced Order Models". Applied Mathematics and Computation 249, pp. 569-596.

Kurganov, A. and E. Tadmor (2000). "New High-Resolution Central Schemes for Nonlinear Conservation Laws and Convection-Diffusion equations". Journal of Computational Physics 160.1, pp. 241-282.

Kurganov, A., S. Noelle, and G. Petrova (2001). "Semidiscrete Central-Upwind Schemes for Hyperbolic Conservation Laws and Hamilton-Jacobi Equations". SIAM Journal on Scientific Computing 23.3, pp. 707-740.

Luke, E., E. Collins, and E. Blades (2012). "A Fast Mesh Deformation Method Using Explicit Interpolation". Journal of Computational Physics 231.2, pp. 586-601.

Marion, J. B. (2013). Classical Dynamics of Particles and Systems. Academic Press, New York.

Mavriplis, D. J. et al. (2009). "Grid Quality and Resolution Issues from the Drag Prediction Workshop Series". Journal of Aircraft 46.3, pp. 935-950.

Mavriplis, D. (1995). "Three-dimensional Multigrid Reynolds-Averaged Navier-Stokes Solver for Unstructured Meshes". AIAA journal 33.3, pp. 445-453.

Moran, J. (1984). An Introduction to Theoretical and Computational Aerodynamics. John Wiley and Sons, New York.

Moreno, R., P. Taylor, and J. Newsom (2012). "A Rigid Horizontal Tail Wind Tunnel Test for High Transonic Mach and High Frequency Unsteady Pressure Acquisition". In: 53rd AIAA Structures, Structural Dynamics and Materials Conference. Honolulu, HI, United States.

Moreno, R., R. Narisetti, and P. Taylor (2015). "A Modification to the Enhanced Correction Factor Technique to Correlate With Experimental Data". In: 56th AIAA Structures, Structural Dynamics, and Materials Conference. 
Nakayama, A (1985). "Characteristics of the Flow Around Conventional and Supercritical Airfoils". Journal of Fluid Mechanics 160, pp. 155-179.

Pulliam, T. H. (1986). "Solution Methods in Computational Fluid Dynamics". Notes for the von Karman Institute For Fluid Dynamics Lecture Series.

Rapún, M.-L., F. Terragni, and J. Vega (2015). "Adaptive POD-based Low-dimensional Modeling Supported by Residual Estimates". International Journal for Numerical Methods in Engineering 104.9, pp. 844-868.

Rapún, M.-L., F Terragni, and J. Vega (2017). "LUPOD: Collocation in POD via LU Decomposition". Journal of Computational Physics 335, pp. 1-20.

Raveh, D. E. (2004). "Identification of Computational-Fluid-Dynamics Based Unsteady Aerodynamic Models for Aeroelastic Analysis". Journal of aircraft 41.3, pp. 620632.

Razak, N., T. Andrianne, and G. Dimitriadis (2011). "Flutter and Stall Flutter of a Rectangular Wing in a Wind Tunnel". AIAA Journal 49.10, pp. 2258-2271.

Rivera, J. J. et al. (1992). "NACA 0012 Benchmark Model Experimental Flutter Results with Unsteady Pressure Distributions". In: Structures, Structural Dynamics, and Materials and Co-located Conferences. AIAA.

Rodden, W. P. Theoretical and computational aeroelasticity, 2011. Crest Publishing, Burbank, California.

Rodden, W. P., P. F. Taylor, and S. C. McIntosh (1998). "Further Refinement of the Subsonic Doublet-Lattice Method". Journal of Aircraft 35.5, pp. 720-727.

Rumsey, C. (2016). Langley Research Center Turbulence Model Resource. URL: https://turbmodels.larc.nasa.gov (visited on 12/01/2016).

Rumsey, C. L. et al. (1996). "Efficiency and Accuracy of Time-accurate Turbulent Navier-Stokes Computations". Computers \& Fluids 25.2, pp. 217-236.

Ryckelynck, D. (2009). "Hyper-reduction of Mechanical Models Involving Internal Variables". International Journal for Numerical Methods in Engineering 77.1, pp. 7589.

Silva, W. and R. Bartels (2004). "Development of Reduced-Order Models for Aeroelastic Analysis and Flutter Prediction Using the CFL3Dv6.0 code". Journal of Fluids and Structures 19.6, pp. $729-745$.

Sousa, V. et al. (2011). "Enhanced Aeroelastic Energy Harvesting by Exploiting Combined Nonlinearities: Theory and Experiment". Smart Materials and Structures 20.9, p. 094007.

Spalart, P. and S. Allmaras (1992). "A One-equation Turbulence Model for Aerodynamic Flows". AIA A Journal 94.

Sutherland, W. (1893). "LII. The Viscosity of Gases and Molecular Force". The London, Edinburgh, and Dublin Philosophical Magazine and Journal of Science 36.223, pp. 507-531.

Sweby, P. K. (1984). "High Resolution Schemes Using Flux Limiters for Hyperbolic Conservation Laws". SIAM Journal on Numerical Analysis 21.5, pp. 995-1011. 
Terragni, F. and J. Vega (2014). "Construction of Bifurcation Diagrams Using POD on the fly". SIAM Journal on Applied Dynamical Systems 13, pp. 330-365.

Terragni, F., E. Valero, and J. Vega (2011). "Local POD plus Galerkin Projection in the Unsteady Lid-driven Cavity Problem". SIAM Journal on Scientific Computing 33, pp. 3538-3561.

Theodorsen, T (1935). General Theory of Aerodynamic Instability and the Mechanism of Flutter. Technical Report 496. NACA.

Tolve, L. (1975). "History of Flight Flutter Testing". In: Proceedings of the 1958 Flight Flutter Testing Symposium. Vol. 385. NASA, pp. 159-166.

Van Leer, B. (1974). "Towards the Ultimate Conservative Difference Scheme. II. Monotonicity and Conservation Combined in a Second-order Scheme". Journal of Computational Physics 14.4, pp. 361-370.

Wesseling, P. (2009). Principles of Computational Fluid Dynamics. Vol. 29. SpringerVerlag, Berlin.

Wright, S. and J. Nocedal (1999). "Numerical Optimization". Springer Science 35.

Xiao, Q. and Q. Zhu (2014). "A Review on Flow Energy Harvesters Based on Flapping Foils". Journal of Fluids and Structures 46.9, pp. 174-191. 
Appendices 


\section{Appendix A}

\section{Inviscid fluid solver Jacobians}

Let $\mathcal{Q}=\{\rho, u, v, w, p\}$ be the state vector joining the primitive variables. The derivative of the inviscid residual $(\mathcal{R})$ with respect to the primitive variables can be obtained as the sum over the faces that conform each cell, such as

$$
\frac{\partial \mathcal{R}}{\partial \mathcal{Q}}=\sum_{f} \frac{\partial \mathcal{R}}{\partial \mathcal{Q}_{f}} \frac{\partial \mathcal{Q}_{f}}{\partial \mathcal{Q}}
$$

where $\frac{\partial \mathcal{Q}_{f}}{\partial \mathcal{Q}}$ depends on the selected face interpolation scheme and $\frac{\partial \mathcal{R}}{\partial \mathcal{Q}_{f}}$ is obtained from the expressions below.

$$
\begin{array}{lll}
\frac{\partial \mathcal{R}_{\rho}}{\partial \rho_{P f}}=\left[\left(\alpha_{f} \phi_{P f}-\omega_{f}\right)\right] ; & \frac{\partial \mathcal{R}_{\rho}}{\partial \rho_{N f}}=-\left[\left(\left(1-\alpha_{f}\right) \phi_{N f}+\omega_{f}\right)\right] ; \\
\frac{\partial \mathcal{R}_{\rho}}{\partial u_{P f}}=\left[\alpha_{f} \rho_{P f}\right] \bar{S}_{f} \cdot \bar{i} ; & \frac{\partial \mathcal{R}_{\rho}}{\partial u_{N f}}=-\left[\left(1-\alpha_{f}\right) \rho_{N f}\right] \bar{S}_{f} \cdot \bar{i} ; \\
\frac{\partial \mathcal{R}_{\rho}}{\partial v_{P f}}=\left[\alpha_{f} \rho_{P f}\right] \bar{S}_{f} \cdot \bar{j} ; & \frac{\partial \mathcal{R}_{\rho}}{\partial v_{N f}}=-\left[\left(1-\alpha_{f}\right) \rho_{N f}\right] \bar{S}_{f} \cdot \bar{j} ; \\
\frac{\partial \mathcal{R}_{\rho}}{\partial w_{P f}}=\left[\alpha_{f} \rho_{P f}\right] \bar{S}_{f} \cdot \bar{k} ; & \frac{\partial \mathcal{R}_{\rho}}{\partial w_{N f}}=-\left[\left(1-\alpha_{f}\right) \rho_{N f}\right] \bar{S}_{f} \cdot \bar{k} ; \\
\frac{\partial \mathcal{R}_{\rho}}{\partial p_{P f}}=\frac{\partial \mathcal{R}_{\rho}}{\partial p_{N f}}=0 ; & \\
\frac{\partial \mathcal{R}_{\rho u}}{\partial \rho_{P f}}=\left[\left(\alpha_{f} \phi_{P f}-\omega_{f}\right) u_{P f}\right] ; & \frac{\partial \mathcal{R}_{\rho u}}{\partial \rho_{N f}}=-\left[\left(\left(1-\alpha_{f}\right) \phi_{N f}+\omega_{f}\right) u_{N f}\right] ; \\
\frac{\partial \mathcal{R}_{\rho u}}{\partial u_{P f}}=\left[\left(\alpha_{f} \phi_{P f}-\omega_{f}\right) \rho_{P f}\right]+\left[\alpha_{f} \rho_{P f} u_{P f}\right] \bar{S} \cdot \bar{i} ; \\
\frac{\partial \mathcal{R}_{\rho u}}{\partial u_{N f}}=-\left[\left(\left(1-\alpha_{f}\right) \phi_{N f}+\omega_{f}\right) \rho_{N f}\right]-\left[\left(1-\alpha_{f}\right) \rho_{N f} u_{N f}\right] \bar{S} \cdot \bar{i} ; \\
\frac{\partial \mathcal{R}_{\rho u}}{\partial v_{P f}}=\left[\alpha_{f} \rho_{P f} u_{P f}\right] \bar{S}_{f} \cdot \bar{j} ;
\end{array}
$$


99

$$
\begin{aligned}
& \frac{\partial \mathcal{R}_{\rho u}}{\partial v_{N f}}=-\left[\left(1-\alpha_{f}\right) \rho_{N f} u_{N f}\right] \bar{S}_{f} \cdot \bar{j} ; \\
& \frac{\partial \mathcal{R}_{\rho u}}{\partial w_{P f}}=\left[\alpha_{f} \rho_{P f} u_{P f}\right] \bar{S}_{f} \cdot \bar{k} ; \\
& \frac{\partial \mathcal{R}_{\rho u}}{\partial w_{N f}}=-\left[\left(1-\alpha_{f}\right) \rho_{N f} u_{N f}\right] \bar{S}_{f} \cdot \bar{k} ; \\
& \frac{\partial \mathcal{R}_{\rho u}}{\partial p_{P f}}=\bar{S}_{f} \cdot \bar{i} \\
& \frac{\partial \mathcal{R}_{\rho u}}{\partial p_{N f}}=-\bar{S}_{f} \cdot \bar{i} \\
& \frac{\partial \mathcal{R}_{\rho v}}{\partial \rho_{P f}}=\left[\left(\alpha_{f} \phi_{P f}-\omega_{f}\right) v_{P f}\right] \\
& \frac{\partial \mathcal{R}_{\rho v}}{\partial \rho_{N f}}=-\left[\left(\left(1-\alpha_{f}\right) \phi_{N f}+\omega_{f}\right) v_{N f}\right] \\
& \frac{\partial \mathcal{R}_{\rho v}}{\partial u_{P f}}=\left[\alpha_{f} \rho_{P f} v_{P f}\right] \bar{S}_{f} \cdot \bar{i} \\
& \frac{\partial \mathcal{R}_{\rho v}}{\partial u_{N f}}=-\left[\left(1-\alpha_{f}\right) \rho_{N f} v_{N f}\right] \bar{S}_{f} \cdot \bar{i} ; \\
& \frac{\partial \mathcal{R}_{\rho v}}{\partial v_{P f}}=\left[\left(\alpha_{f} \phi_{P f}-\omega_{f}\right) \rho_{P f}\right]+\left[\alpha_{f} \rho_{P f} v_{P f}\right] \bar{S} \cdot \bar{j} ; \\
& \frac{\partial \mathcal{R}_{\rho v}}{\partial v_{N f}}=-\left[\left(\left(1-\alpha_{f}\right) \phi_{N f}+\omega_{f}\right) \rho_{N f}\right]-\left[\left(1-\alpha_{f}\right) \rho_{N f} v_{N f}\right] \bar{S} \cdot \bar{j} \\
& \frac{\partial \mathcal{R}_{\rho v}}{\partial w_{P f}}=\left[\alpha_{f} \rho_{P f} v_{P f}\right] \bar{S}_{f} \cdot \bar{k} \\
& \frac{\partial \mathcal{R}_{\rho v}}{\partial w_{N f}}=-\left[\left(1-\alpha_{f}\right) \rho_{N f} v_{N f}\right] \bar{S}_{f} \cdot \bar{k} ; \\
& \frac{\partial \mathcal{R}_{\rho v}}{\partial p_{P f}}=\bar{S}_{f} \cdot \bar{j} \\
& \frac{\partial \mathcal{R}_{\rho v}}{\partial p_{N f}}=-\bar{S}_{f} \cdot \bar{j} \\
& \frac{\partial \mathcal{R}_{\rho w}}{\partial \rho_{P f}}=\left[\left(\alpha_{f} \phi_{P f}-\omega_{f}\right) v_{P f}\right] \\
& \frac{\partial \mathcal{R}_{\rho w}}{\partial \rho_{N f}}=-\left[\left(\left(1-\alpha_{f}\right) \phi_{N f}+\omega_{f}\right) v_{N f}\right] \text {; } \\
& \frac{\partial \mathcal{R}_{\rho w}}{\partial u_{P f}}=\left[\alpha_{f} \rho_{P f} w_{P f}\right] \bar{S}_{f} \cdot \bar{i} \\
& \frac{\partial \mathcal{R}_{\rho w}}{\partial u_{N f}}=-\left[\left(1-\alpha_{f}\right) \rho_{N f} w_{N f}\right] \bar{S}_{f} \cdot \bar{i} \text {; } \\
& \frac{\partial \mathcal{R}_{\rho w}}{\partial v_{P f}}=\left[\alpha_{f} \rho_{P f} w_{P f}\right] \bar{S}_{f} \cdot \bar{j} ; \\
& \frac{\partial \mathcal{R}_{\rho w}}{\partial v_{N f}}=-\left[\left(1-\alpha_{f}\right) \rho_{N f} w_{N f}\right] \bar{S}_{f} \cdot \bar{j} ;
\end{aligned}
$$




$$
\begin{aligned}
& \frac{\partial \mathcal{R}_{\rho w}}{\partial w_{P f}}=\left[\left(\alpha_{f} \phi_{P f}-\omega_{f}\right) \rho_{P f}\right]+\left[\alpha_{f} \rho_{P f} w_{P f}\right] \bar{S} \cdot \bar{k} \\
& \frac{\partial \mathcal{R}_{\rho w}}{\partial w_{N f}}=-\left[\left(\left(1-\alpha_{f}\right) \phi_{N f}+\omega_{f}\right) \rho_{N f}\right]-\left[\left(1-\alpha_{f}\right) \rho_{N f} w_{N f}\right] \bar{S} \cdot \bar{k} ; \\
& \frac{\partial \mathcal{R}_{\rho w}}{\partial p_{P f}}=\bar{S}_{f} \cdot \bar{k} \\
& \frac{\partial \mathcal{R}_{\rho w}}{\partial p_{N f}}=-\bar{S}_{f} \cdot \bar{k} \\
& \frac{\partial \mathcal{R}_{\rho E}}{\partial \rho_{P f}}=\frac{1}{2}\left[\left(\alpha_{f} \phi_{P f}-\omega_{f}\right)\left(u_{P f}^{2}+v_{P f}^{2}+w_{P f}^{2}\right)\right] ; \\
& \frac{\partial \mathcal{R}_{\rho E}}{\partial \rho_{N f}}=-\frac{1}{2}\left[\left(\left(1-\alpha_{f}\right) \phi_{N f}+\omega_{f}\right)\left(u_{N f}^{2}+v_{N f}^{2}+w_{N f}^{2}\right)\right] \\
& \frac{\partial \mathcal{R}_{\rho E}}{\partial u_{P f}}=\left[\left(\alpha_{f} \phi_{P f}-\omega_{f}\right) \rho_{P f} u_{P f}\right]+\alpha_{f}\left[\frac{\gamma p_{P f}}{\gamma-1}+\frac{\rho_{P f}}{2}\left(u_{P f}^{2}+v_{P f}^{2}+w_{P f}^{2}\right)\right] \bar{S}_{f} \cdot \bar{i} ; \\
& \frac{\partial \mathcal{R}_{\rho E}}{\partial u_{N f}}=-\left[\left(\left(1-\alpha_{f}\right) \phi_{N f}-\omega_{f}\right) \rho_{N f} u_{N f}\right] \\
& -\left(1-\alpha_{f}\right)\left[\frac{\gamma p_{N f}}{\gamma-1}+\frac{\rho_{N f}}{2}\left(u_{N f}^{2}+v_{N f}^{2}+w_{N f}^{2}\right)\right] \bar{S}_{f} \cdot \bar{i}_{;} \\
& \frac{\partial \mathcal{R}_{\rho E}}{\partial v_{P f}}=\left[\left(\alpha_{f} \phi_{P f}+\omega_{f}\right) \rho_{P f} u_{P f}\right]+\alpha_{f}\left[\frac{\gamma p_{P f}}{\gamma-1}+\frac{\rho_{P f}}{2}\left(v_{P f}^{2}+v_{P f}^{2}+w_{P f}^{2}\right)\right] \bar{S}_{f} \cdot \bar{j} ; \\
& \frac{\partial \mathcal{R}_{\rho E}}{\partial v_{N f}}=-\left[\left(\left(1-\alpha_{f}\right) \phi_{N f}+\omega_{f}\right) \rho_{N f} v_{N f}\right] \\
& -\left(1-\alpha_{f}\right)\left[\frac{\gamma p_{N f}}{\gamma-1}+\frac{\rho_{N f}}{2}\left(v_{N f}^{2}+v_{N f}^{2}+w_{N f}^{2}\right)\right] \bar{S}_{f} \cdot \bar{j} ; \\
& \frac{\partial \mathcal{R}_{\rho E}}{\partial w_{P f}}=\left[\left(\alpha_{f} \phi_{P f}-\omega_{f}\right) \rho_{P f} u_{P f}\right]+\alpha_{f}\left[\frac{\gamma p_{P f}}{\gamma-1}+\frac{\rho_{P f}}{2}\left(w_{P f}^{2}+v_{P f}^{2}+w_{P f}^{2}\right)\right] \bar{S}_{f} \cdot \bar{k} ; \\
& \frac{\partial \mathcal{R}_{\rho E}}{\partial w_{N f}}=-\left[\left(\left(1-\alpha_{f}\right) \phi_{N f}+\omega_{f}\right) \rho_{N f} w_{N f}\right] \\
& -\left(1-\alpha_{f}\right)\left[\frac{\gamma p_{N f}}{\gamma-1}+\frac{\rho_{N f}}{2}\left(w_{N f}^{2}+v_{N f}^{2}+w_{N f}^{2}\right)\right] \bar{S}_{f} \cdot \bar{k} \\
& \frac{\partial \mathcal{R}_{\rho E}}{\partial p_{P f}}=\left[\left(\alpha_{f} \phi_{P f}-\omega_{f}\right) \frac{1}{\gamma-1}\right]+\alpha_{f}\left[u_{P f} \bar{S}_{f} \cdot \bar{i}+v_{P f} \bar{S}_{f} \cdot \bar{j}+w_{P f} \bar{S}_{f} \cdot \bar{k}\right] \\
& \frac{\partial \mathcal{R}_{\rho E}}{\partial p_{N f}}=-\left[\left(\left(1-\alpha_{f}\right) \phi_{N f}+\omega_{f}\right) \frac{1}{\gamma-1}\right]-\left(1-\alpha_{f}\right)\left[u_{N f} \bar{S}_{f} \cdot \bar{i}+v_{N f} \bar{S}_{f} \cdot \bar{j}+w_{N f} \bar{S}_{f} \cdot \bar{k}\right] .
\end{aligned}
$$




\section{Appendix B}

\section{Theodorsen unsteady aerodynamics}

The classical solution for the subsonic potential flow aerodynamic forces over an airfoil with a control surface is due to Theodorsen (Theodorsen, 1935).

$$
\begin{gathered}
L=\pi \rho b^{2}\left(\ddot{h}-a b \ddot{\alpha}-\frac{T_{1}}{\pi} b \ddot{\beta}-\frac{T_{4}}{\pi} v \dot{\beta}\right) \\
+2 \pi \rho v b C(k) f(t), \\
M_{\alpha}=-\rho b^{2}\left[-a \pi b \ddot{h}+\pi\left(\frac{1}{2}-a\right) v b \dot{\alpha}+\pi b^{2}\left(\frac{1}{8}+a^{2}\right) \ddot{\alpha}+T_{15} v^{2} \beta+T_{16} v b \dot{\beta}+2 T_{13} b^{2} \ddot{\beta}\right] \\
+2 \pi \rho v b^{2}\left(a+\frac{1}{2}\right) C(k) f(t), \\
M_{\beta}=-\rho b^{2}\left[-T_{1} b \ddot{h}+T_{17} v b \dot{\alpha}+2 T_{13} b^{2} \ddot{\alpha}+\frac{T_{18}}{\pi} v^{2} \beta+\frac{T_{19}}{\pi} v b \ddot{\beta}-\frac{T_{3}}{\pi} b^{2} \ddot{\beta}\right] \\
-\rho v b^{2} T_{12}\left(a+\frac{1}{2}\right) C(k) f(t),
\end{gathered}
$$

with

$$
f(t)=\left\{v \alpha+\dot{h}+b\left(\frac{1}{2}-a\right) \dot{\alpha}+\frac{T_{10}}{\pi} v \beta+\frac{T_{11}}{2 \pi} b \dot{\beta}\right\}
$$

In each of the above equations there are two types of contributions. The ones multiplied by $\mathrm{C}(\mathrm{k})$ account for the circulatory terms (i.e. those needed to fulfill the Kutta's hypothesis of zero pressure discontinuity at the trailing edge) whereas the nonmultiplied by $\mathrm{C}(\mathrm{k})$ account for the instantaneous velocity of each individual part in the whole of the airfoil.

$\mathrm{C}(\mathrm{k})$ is known as the Theodorsen's function and is defined by

$$
C(k)=\frac{\int_{1}^{\infty} \frac{x_{o}}{\sqrt{x_{o}^{2}-1}} e^{-i k x_{o}} \mathrm{~d} x_{\circ}}{\int_{1}^{\infty} \frac{x_{o}+1}{\sqrt{x_{o}^{2}-1}} e^{-i k x_{o}} \mathrm{~d} x_{\circ}}
$$


or, expressed in terms of the Hankel's functions, as

$$
C(k)=\frac{H_{1}^{(2)}(k)}{H_{1}^{(2)}(k)+i H_{0}^{(2)}(k)},
$$

where $k=\frac{\omega b}{v}$ is a dimensionless frequency, known as reduced frequency.

$T_{i}$ correspond to geometrical constants of the airfoil (Theodorsen, 1935).

$$
\begin{aligned}
T_{1} & =\frac{-1}{3}\left(1+\sqrt{1-c^{2}}\left(2+c^{2}\right)\right)+c \arccos (c) \\
T_{3} & =-\left(\frac{1}{8}+c^{2}\right)(\arccos 2(c))+\frac{1}{4} c \sqrt{1-c^{2}} \arccos (c)\left(7+2 c^{2}\right)-\frac{1}{8}\left(1-c^{2}\right)\left(5 c^{2}+4\right) \\
T_{4} & =-\arccos (c)+c \sqrt{1-c^{2}} \\
T_{5} & =-\left(1-c^{2}\right)-\arccos { }^{2}(c)+2 c \sqrt{1-c^{2}} \arccos (c) \\
T_{7} & =-\left(\frac{1}{8}+c^{2}\right) \arccos (c)+\frac{1}{8} c \sqrt{1-c^{2}}\left(7+2 c^{2}\right) \\
T_{8} & =-\frac{1}{3} \sqrt{1-c^{2}}\left(2 c^{2}+1\right)+c \arccos (c) \\
T_{10} & =\sqrt{1-c^{2}}+\arccos (c) \\
T_{11} & =\arccos (c)(1-2 c)+\sqrt{1-c^{2}}(2-c) \\
T_{12} & =\sqrt{1-c^{2}}(2+c)-\arccos (c)(2 c+1) \\
T_{13} & =-\frac{1}{2}\left(T_{7}+(c-a) T_{1}\right) \\
T_{14} & =\frac{1}{16}+\frac{1}{2} a c \\
T_{15} & =T_{4}+T_{1} 0 \\
T_{16} & =T_{1}-T_{8}-(c-a) T_{4}+\frac{T_{11}}{2} \\
T_{17} & =-\frac{1}{3} \sqrt[3]{\left(1-c^{2}\right)^{2}}-T_{1}-\frac{T_{4}}{2} \\
T_{18} & =T_{5}-T_{4} T_{10} \\
T_{19} & =-\frac{T_{4}}{2} T_{11}
\end{aligned}
$$

Theodorsen equations meant a big advance to the representation of unsteady aerodynamics. One of their greatest advantages, the representation of the aerodynamics in the frequency domain, making it easier the study of the linear stability of the airfoil, is also one of their biggest disadvantages, as it limits any possible study to the frequency domain.

With the use of Fourier synthesis, the formulation can be extended to the time domain, and therefore be valid for airfoil arbitrary motions. The non-circulatory terms are instantaneous and independent of the motion's frequency, so they can be translated to the time domain without any further considerations.

Focusing our attention on the circulatory terms, whose frequency dependency is kept through $\mathrm{C}(\mathrm{k})$, and transforming $f(t)$ to the frequency domain, then the circulatory 
terms can be expressed in time domain as

$$
\Delta_{c}(t)=\frac{1}{2 \pi} \int_{-\infty}^{\infty} C\left(\frac{\omega b}{v}\right) f(\omega) e^{i \omega t} \mathrm{~d} \omega
$$

Considering the response to a step in any given term of $f(t), \chi_{o}$,equation (B.5) could be reduced to

$$
\left.\Delta_{c}(s)\right|_{\Delta_{o}}=\chi_{o}\left[\frac{1}{2 \pi} \int_{-\infty}^{\infty} \frac{C(k)}{i k} e^{i k s} \mathrm{dk}\right]
$$

where the time, $t$, has been substituted by the dimensionless time $s=\frac{v t}{b}$.

The term in brackets in equation (B.6) is known as the Wagner function. It is relatively simple but is not expressible in terms of elementary functions.

Making use of the Duhammel's superposition, we may conclude that

$$
\Delta_{c}(s)=f(0) \phi(s)+\int_{0}^{s} \frac{\mathrm{d} f(\sigma)}{\mathrm{d} \sigma} \phi(s-\sigma) \mathrm{d} \sigma
$$




\section{Appendix C}

\section{Inviscid aerodynamic solver validation}

The inviscid CFD code has been validated in the subsonic range using pitching motions against the analytical expressions (Theodorsen, 1935). In particular, for $M=0.2$ at sea level, and a forced pitching amplitude of $0.01 \mathrm{rad}$ at $2.5 \mathrm{~Hz}$ (reduced frequency $k=0.116$ ), a lift coefficient $C_{l \alpha}=5.2+0.3 \mathrm{i}$ is obtained with the CFD code, while the analytical prediction by Theodorsen and Center is $C_{l \alpha}=5.2+0.1 \mathrm{i}$. The time history using Theodorsen analytical expressions has been obtained making use of equation (B.7). A comparison between the two methods can be seen in figure C.1, where the agreement is excellent both in amplitude and phase shift of the incremental portion. It must be noted, however, that Theodorsen formulation is not capable of capturing thickness effects, which might explain the shift in the steady portion of the lift. It is also shown in the same figure the comparison between the results obtained for pitch motions in which the mesh is rotated rigidly with the airfoil, and the results obtained when the mesh deforms to accommodate the airfoil motion. The small difference between the two results validates the scheme used to deform the mesh and integrate the fluid equations in the case of deforming meshes.

If the oscillation frequency is increased to $10 \mathrm{~Hz}$ (reduced frequency $k=0.464$ ), while staying at the same conditions, the agreement is still excellent.

Validation in the transonic regime, for small oscillations, is made against results reported by Batina (Batina, 1985). For $M=0.8$, with $k=0.115$, the CFD code produces a lift coefficient due to pitch $C_{l \alpha}=5.8-3.2 \mathrm{i}$ while Batina reports a value $C_{l \alpha}=5.7-4.3 \mathrm{i}$. With $M=0.8$ and a larger reduced frequency, $k=0.462$, the corresponding values for $C_{l \alpha}$ obtained with the CFD code and reported by Batina are $4.1+0.1 \mathrm{i}$ and $4.5+0.2 \mathrm{i}$, respectively.

With the results presented above, the code has been deemed suitable to demonstrate the impact of the incorporation of the adaptive POD based ROM into the unsteady aerodynamic simulations for inviscid flow. 


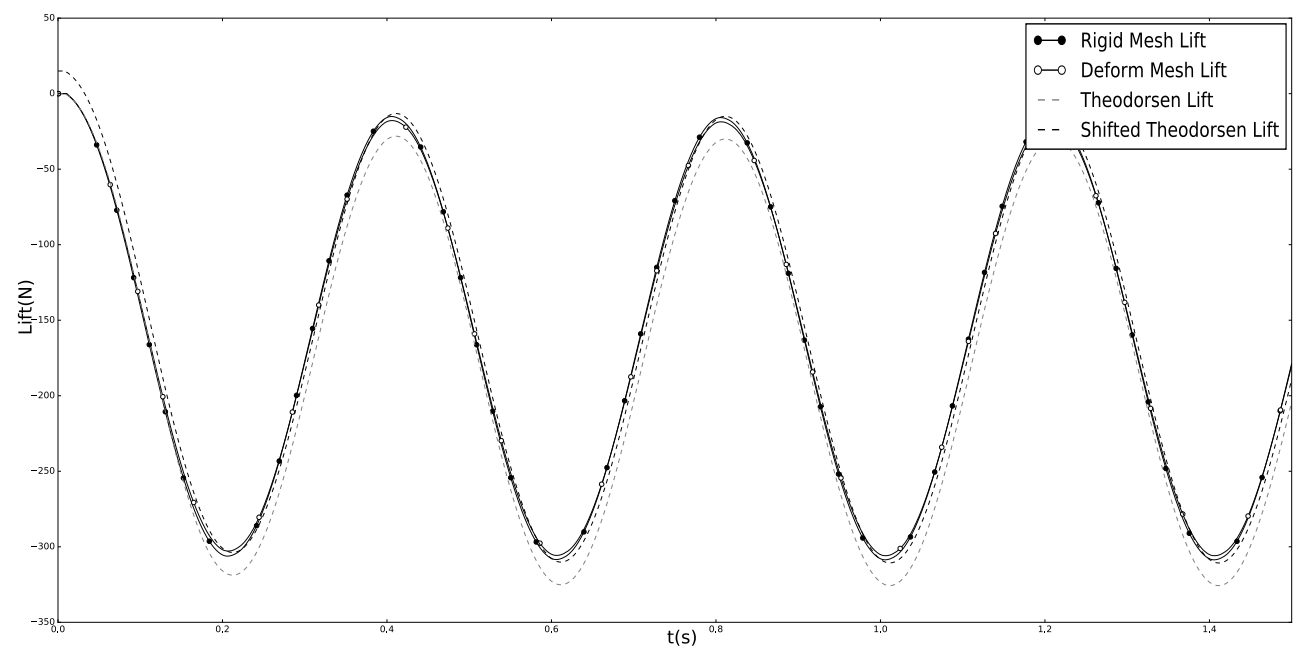

Figure C.1: Lift comparison between the CFD solver and Theodorsen analytical expressions

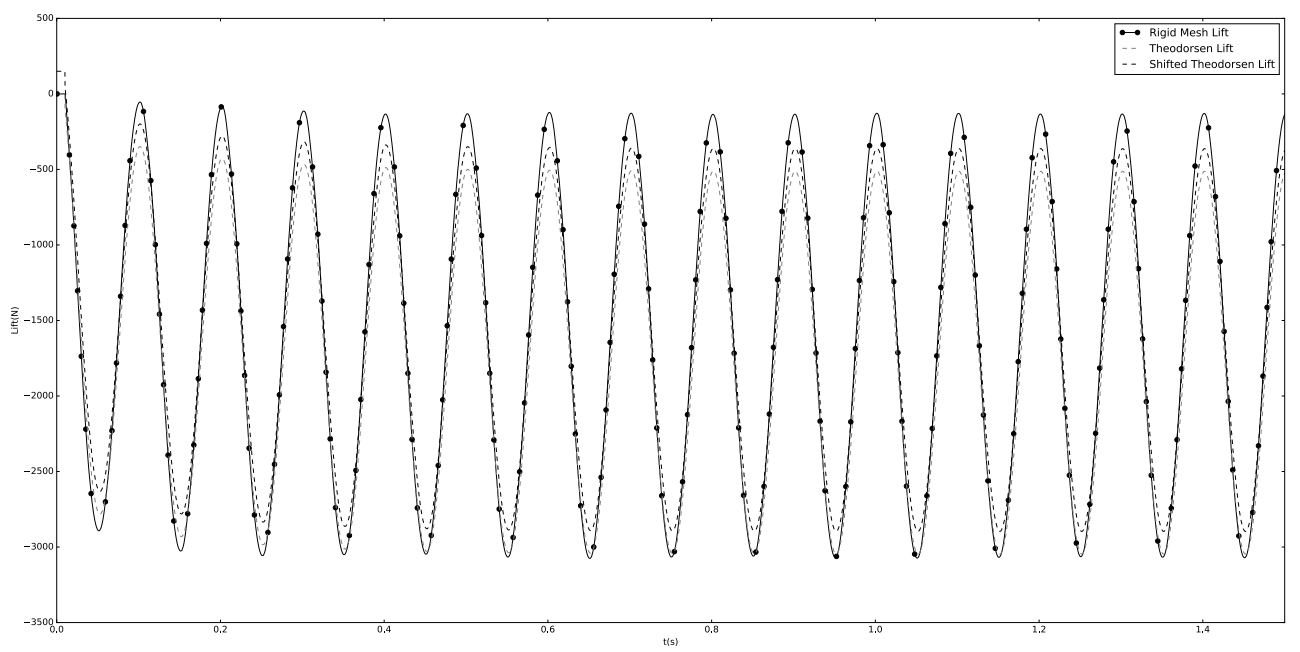

Figure C.2: Lift comparison between the CFD solver and Theodorsen analytical expressions 


\section{Appendix D}

\section{Viscous aerodynamic solver validation}

The numerical solver has been validated against several of the turbulence benchmark cases published by NASA in (Rumsey, 2016). The validation results against the $2 \mathrm{D}$ Zero pressure gradient flat plate (2DZP), the 2D Airfoil near wake (2DANW), the 2D NACA 0012 airfoil (2DN00) and the 2D NACA 4412 airfoil trailing edge separation (2DN44) cases can be seen in the following sections.

Comparison against transonic available data for the 2D NACA 0012 airfoil as well as against the unsteady data generated with the already validated inviscid code are also presented in the sections below.

With the presented results, the code has been deemed suitable to demonstrate the impact of incorporating the adaptive POD based ROM into the unsteady aerodynamic simulations for viscous turbulent flow.

\section{D.1 2D Zero pressure gradient flat plate (2DZP)}

This is a simple case to test the development of viscous boundary layers. It simulates the flow over a flat plate with no pressure gradient imposed. The turbulent flat plate case was run at $\mathrm{M}=0.2$, at a Reynolds number of $\mathrm{Re}=5$ million based on length "1" of the grid. The body reference length is 2 units. Because the solid wall of the grid extended from $\mathrm{x}=0$ to $\mathrm{x}=2$, this means that the $R e_{x}$ at $x=1$ was 5 million, and $R e_{x}$ at $x=2$ (the downstream end of the plate) was 10 million. Figure D.1 shows the layout of the simple flat plate grids used for this study, along with the boundary conditions.

The NASA provided CGNS unstructured quad grid has been used for the $2 \times 273 \times 193$ version ( $2 \times 225$ points on the solid plate). It is the second to finest mesh, with a minimum spacing at the wall of $y=10^{-6}$, resulting in an approximate average $y^{+}$equal to 0.2 . A detail of the mesh, for the second to coarsest mesh, can be seen in figure D.2. The case has been run initially with a first order spatially accurate differencing scheme. A comparison between the obtained velocity profile at $x=0.97$ and the theoretical curve, 


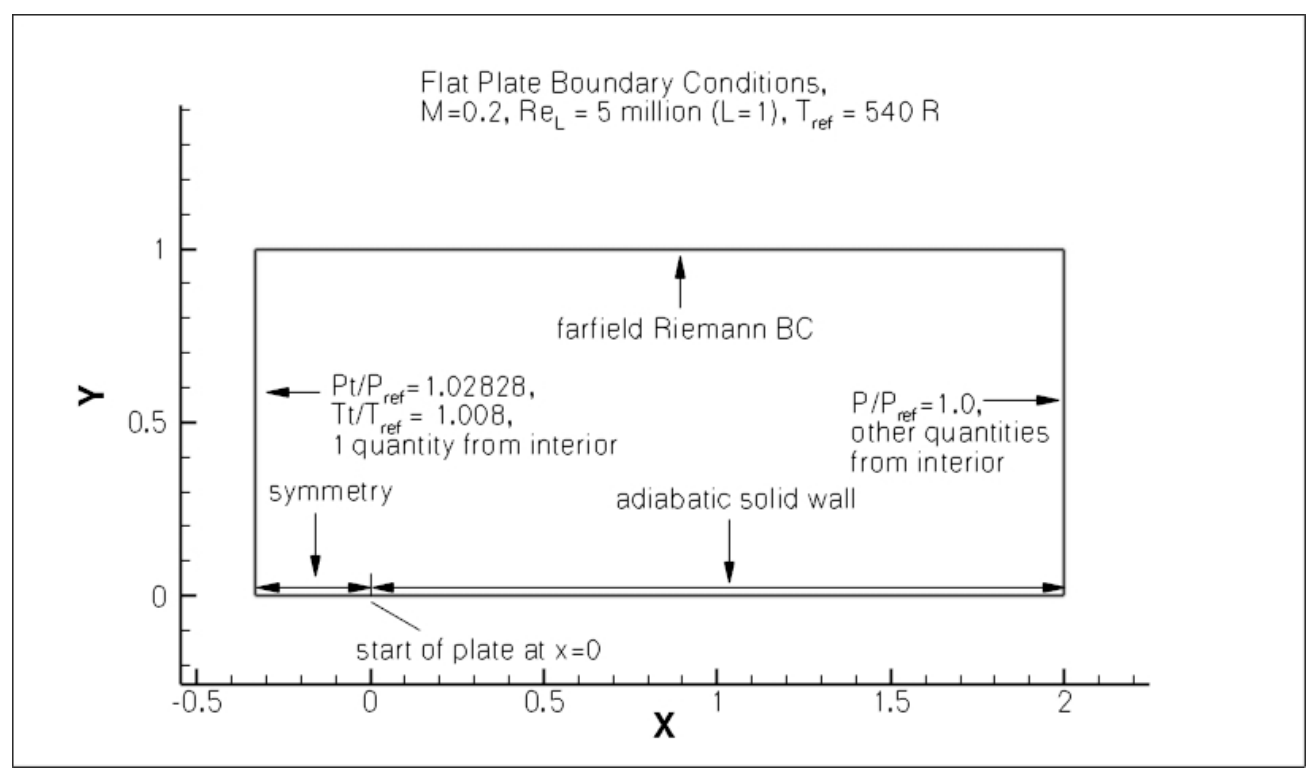

Figure D.1: 2D Zero pressure gradient flat plate geometry description

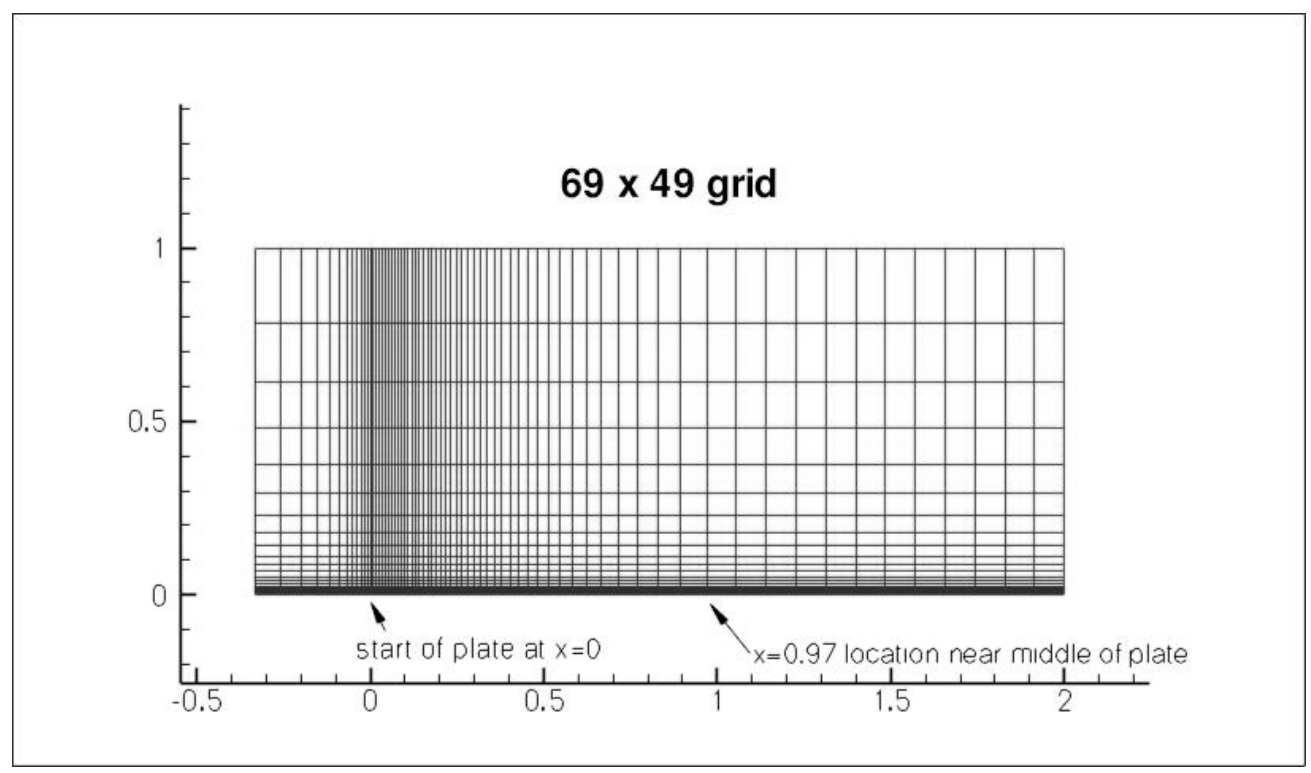

Figure D.2: 2DZP Mesh Description

based on Coles' mean velocity profile along with a van Driest damping near the wall (Bardina et al., 1997), can be seen in figure D.3. The figure shows that the CFD solver is too dissipative and the upstream velocity is recovered much farther away from the wall than expected, given the case conditions. Upon further investigation, it has been seen that the increased apparent dissipation is due to the combination between the first order convection differencing scheme and the KNP additional diffusion in equation (3.9), based on the volumetric flux $\omega_{f}$. This additional diffusion is needed to stabilize the numerical scheme, and can not be made null without jeopardizing the overall solution convergence. However, by multiplying the value of $\omega_{f}$ by 0.1 , even if it is only for the terms corresponding to the momentum conservation equation and the turbulence 
variable transport, the resulting values approach the expected theoretical values.

A further improvement can be obtained by incorporating a second order convection differencing scheme, together with a Van Leer limiter, in the same momentum and turbulence variable equations, while maintaining the original volumetric diffusion values (see black circular markers in figure D.3). With this approach, the comparison is very good, within the uncertainty band from the different theories.

Finally, for consistency, a second order convection differencing scheme, with a Van Leer limiter, has been used for all the convection terms in the fluid governing equations. This is the baseline configuration for all the viscous turbulent calculations presented in this thesis.

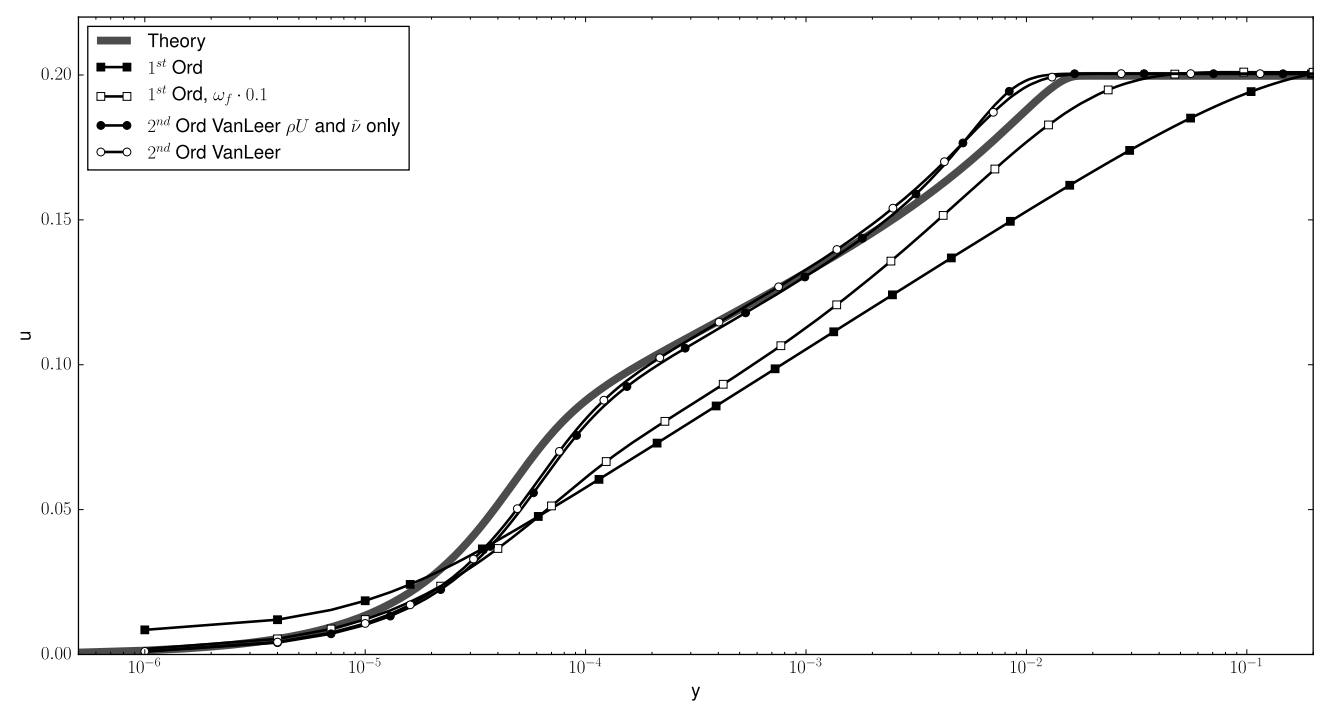

Figure D.3: 2DZP Comparison between theoretical and CFD Obtained boundary layer velocity profile at $\mathrm{x}=0.97$, with different convection differencing schemes

\section{D.2 2D Airfoil near wake (2DANW)}

The 2-D airfoil near wake case involves the flow over a non-symmetric $10 \%$ thick conventional airfoil (referred to as the "Model A-airfoil"). Wake characteristics were measured (Nakayama, 1985) with the airfoil at $0^{\circ}$ angle of attack, $\mathrm{M}=0.088$ and $R e_{c}$ equal to 1.2 million. Figure D.4 shows the domain geometry and the utilized boundary conditions.

The NASA provided CGNS unstructured quad grid has been used for the $2 \times 561 \times 97$ version (2x257 points on the airfoil surface). A detail of the mesh can be seen in figure D.5. It is the third to finest (and coarsest) mesh, with a minimum spacing at the wall of $y=10^{-5}$, resulting in an approximate average $y^{+}$equal to 0.8 . The CFD code has been run with $\mathrm{M}=0.15$ which still remains in the essentially incompressible regime.

The obtained speeds and pressure maps can be seen in figure D.11. The horizontal fluid speed value in the wake obtained with the CFD code is compared against the experimental data for $y=0$ in figure D.6. The agreement is very good in the vicinity of the trailing edge, where the mesh is finer. The fluid speed seems to recover earlier in 


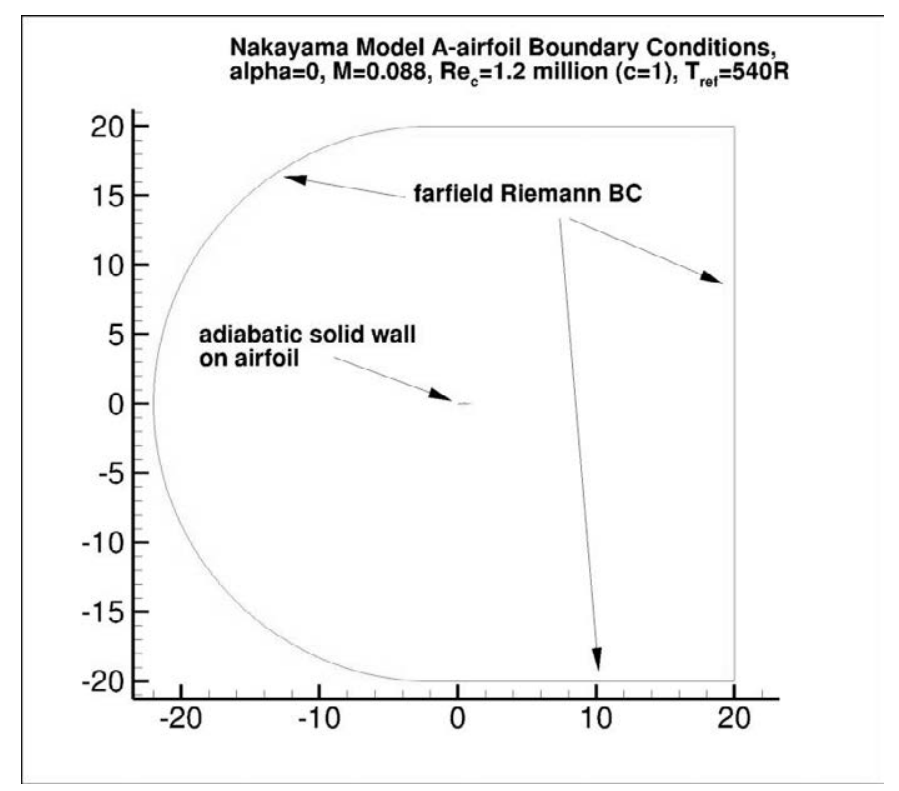

Figure D.4: 2DANW Boundary conditions
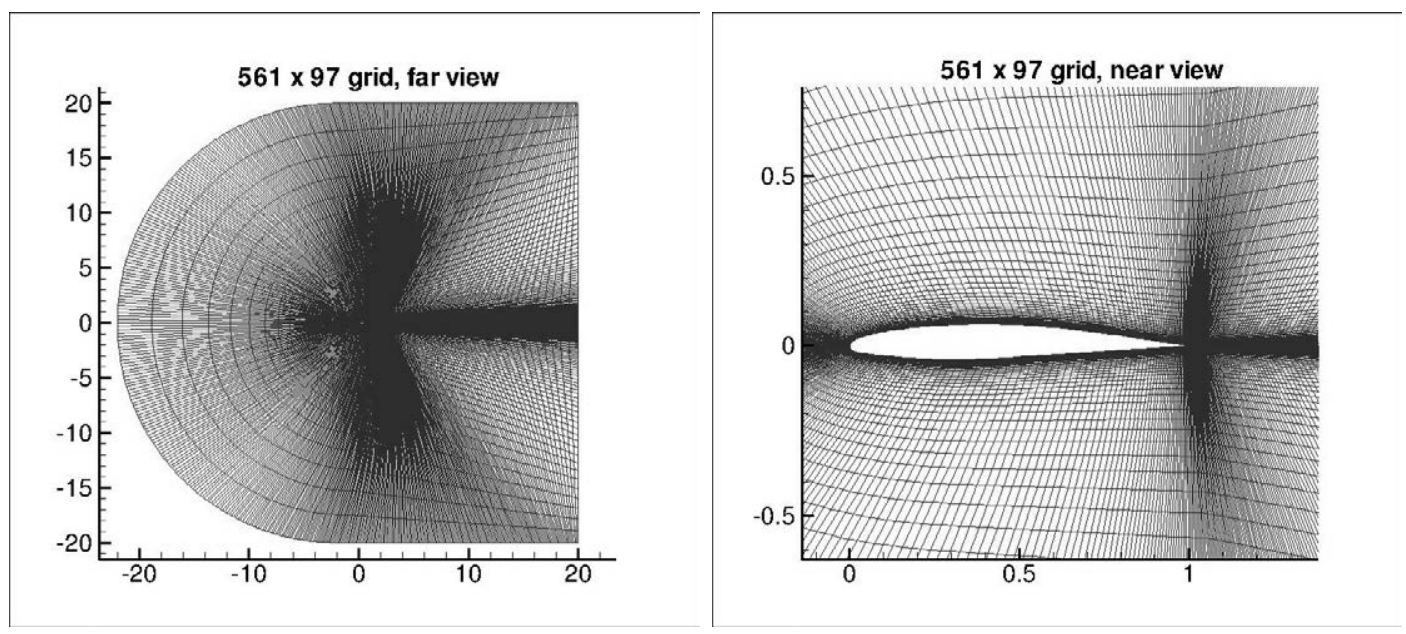

Figure D.5: 2DANW Mesh detail

the CFD analysis compared with the experiment, but it could be attributed to lack of resolution in the mesh as data from further downstream is analyzed. This is consistent with the mesh sensitivity results documented for NASA's CFL3D.

In a similar way, the fluid horizontal speed profiles at three different measured constant $\mathrm{x}$ locations are compared against analytical results in figure D.7. The agreement is very good also in the vicinity of the airfoil, and degrades as the comparison moves further along the wake. As in the previous comparison, this evolution is consistent with mesh sensitiviy results for NASA's CFL3D. 


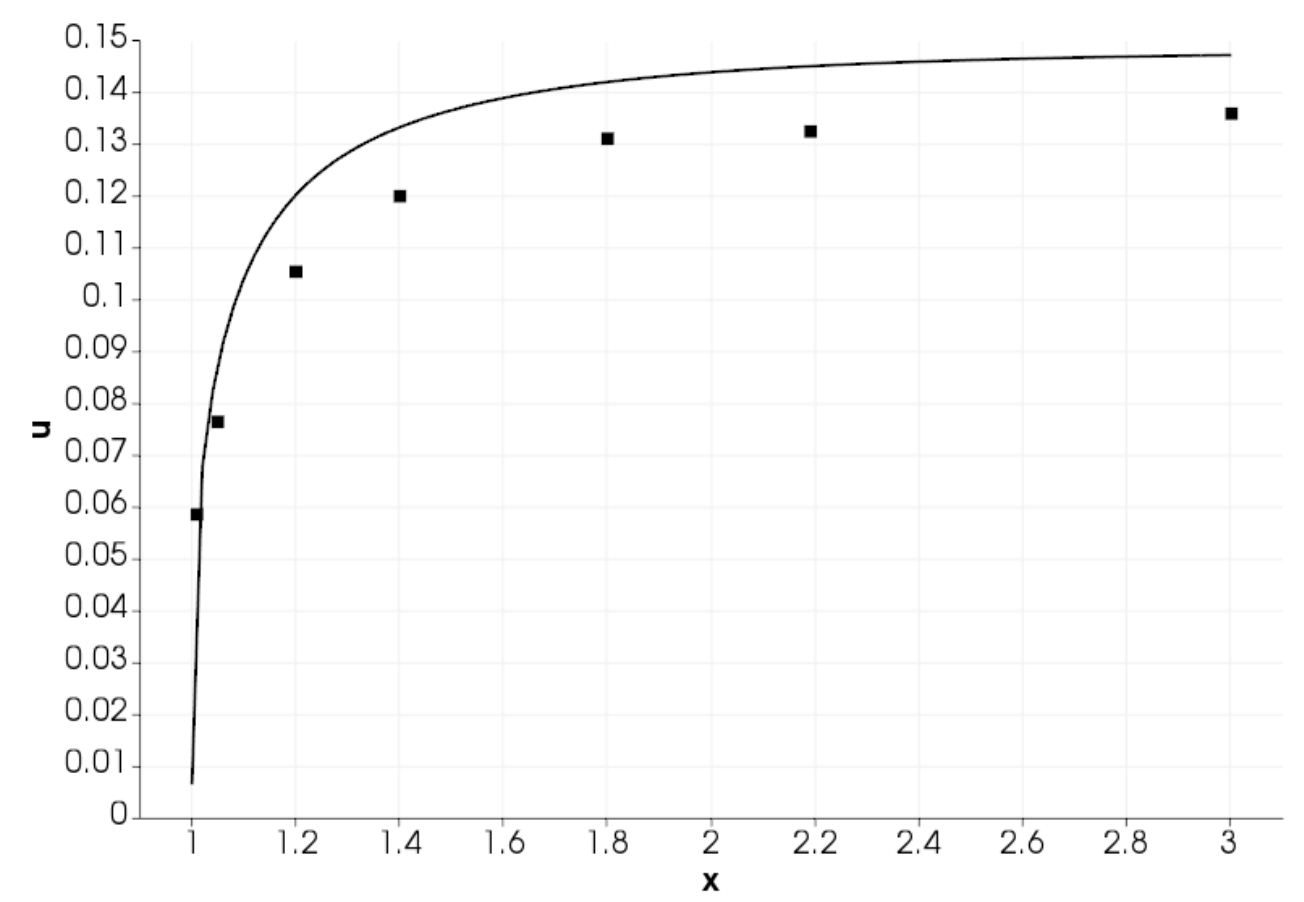

Figure D.6: Velocity profiles at different $\mathrm{x}$ locations in the airfoil wake at $\mathrm{y}=0$

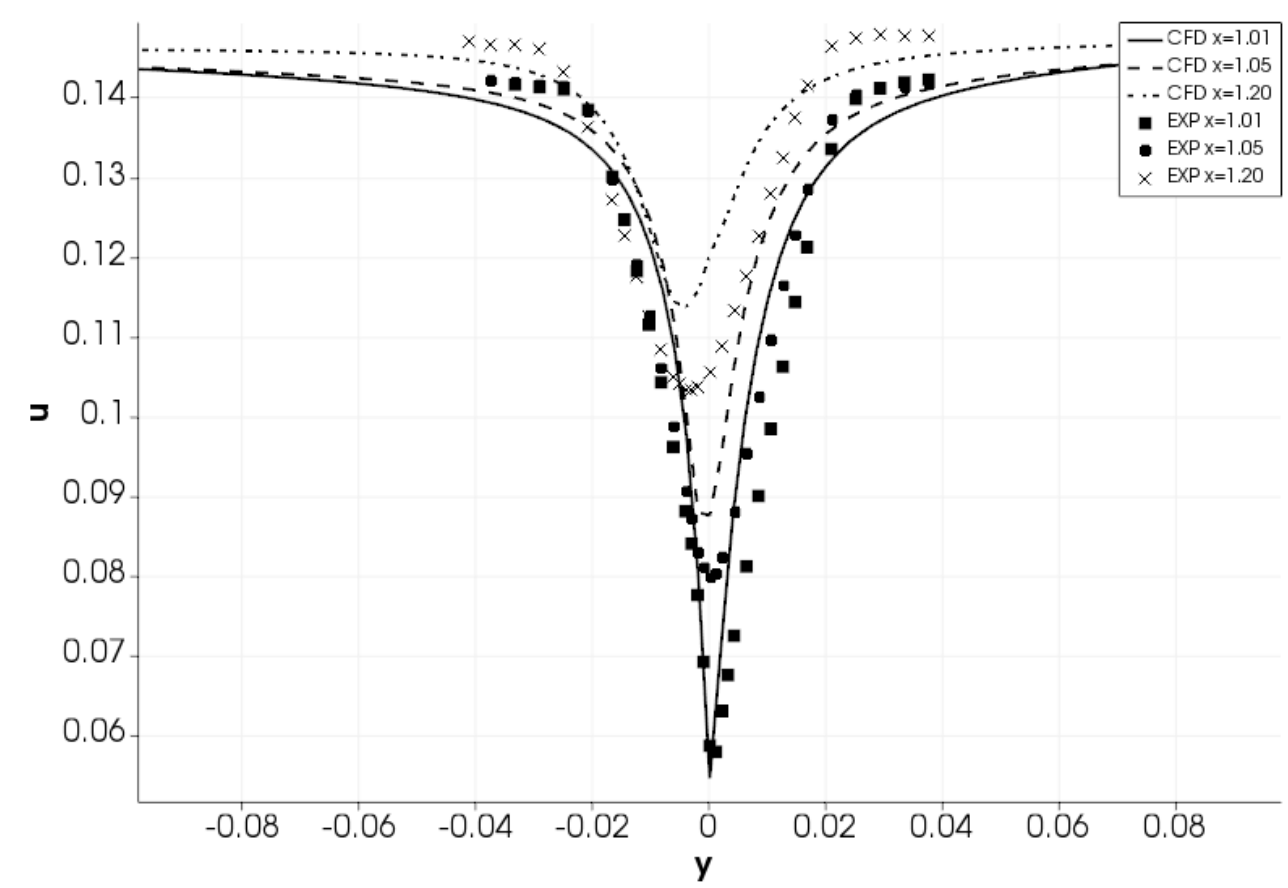

Figure D.7: Velocity profiles at different $\mathrm{x}$ locations in the airfoil wake 


\section{D.3 2D NACA 4412 airfoil trailing edge separation (2DN44)}

The 2-D NACA 4412 airfoil trailing edge separation involves the flow over a NACA 4412 airfoil (cambered, $12 \%$ thickness) at 13.87 degrees of angle of attack. Flow speed measurements were taken (Coles and Wadcock, 1979) at $\mathrm{M}=0.090$ and $R e_{c}$ equal to 1.52 million. Figure D.4 shows the domain geometry and the utilized boundary conditions.

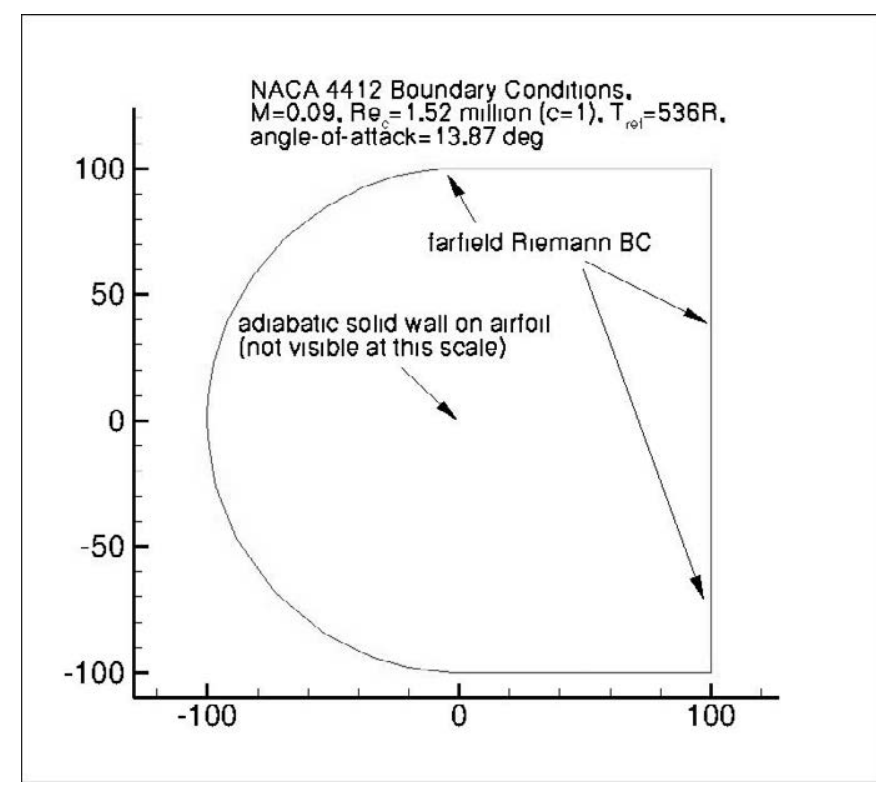

Figure D.8: 2DN44 Boundary conditions

The NASA provided CGNS unstructured quad grid has been used for the $2 \times 897 \times 257$ version ( $2 \times 513$ points on the airfoil surface). It is the second to finest mesh, with a minimum spacing at the wall of $y=4 \cdot 10^{-6}$, resulting in an approximate average $y^{+}$ equal to 0.4. A detail of the mesh, for a mesh that is exactly every-other-point of the one utilized, can be seen in figure D.9. The CFD code has been run with $M=0.10$ which still remains in the essentially incompressible regime.
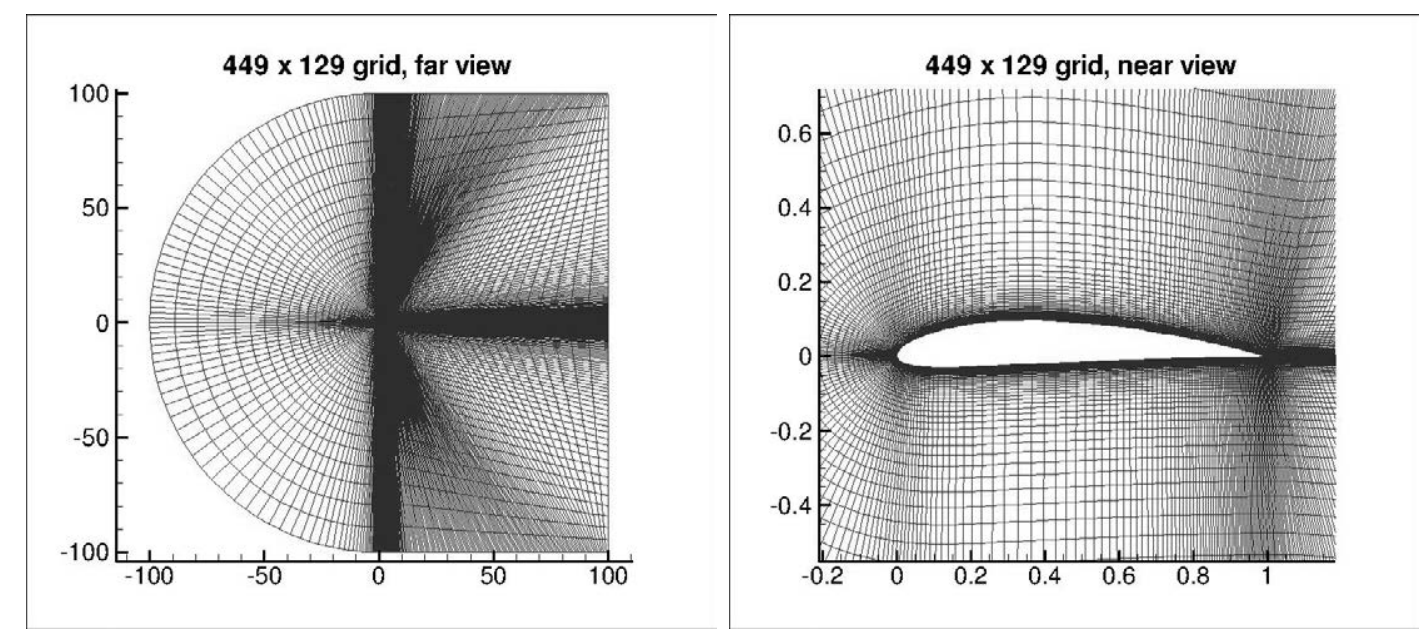

Figure D.9: 2DN44 Boundary conditions 
The experimental data was used to create a velocity contour plot and streamlines, that can be seen together with the ones generated by the CFD created in this thesis in figure D.12. The agreement is excellent between the CFD runs and the experimental data.

The flow speeds along three different lines (first, third and last visibles in figure D.12left) are compared for the experimental data and the CFD obtained results in figure D.10. The agreement is excellent, in line with NASA's CFL3D reproduced data.

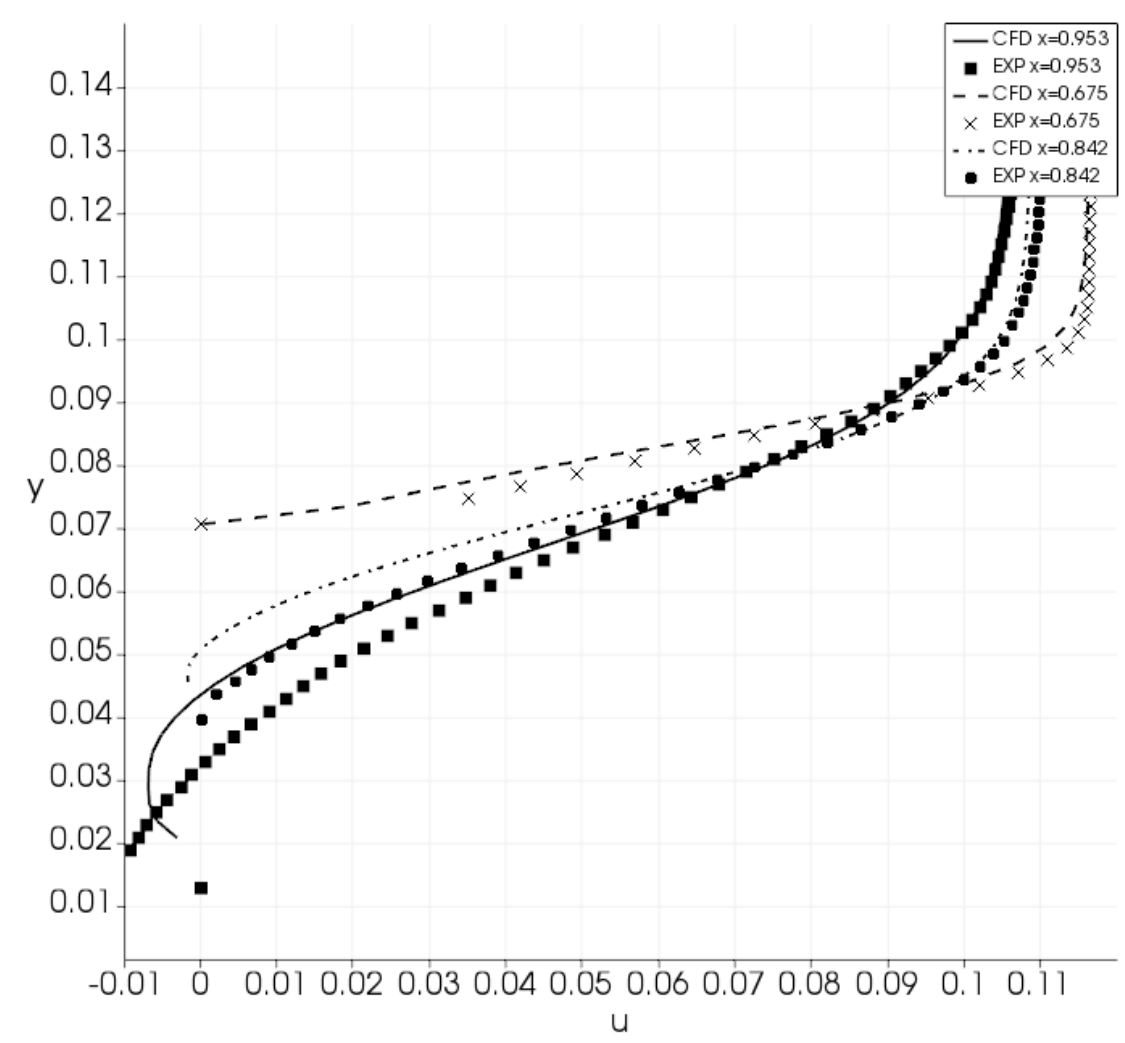

Figure D.10: 2DN44 Velocity profiles along different lines in the vicinity of the trailing edge 

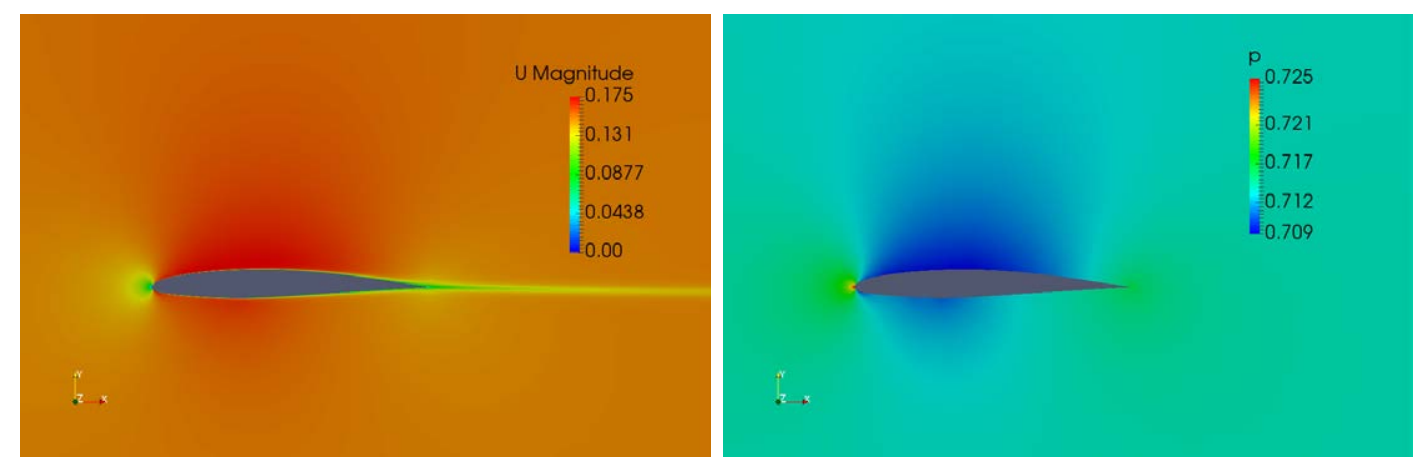

Figure D.11: 2DANW CFD Obtained pressure and velocity maps
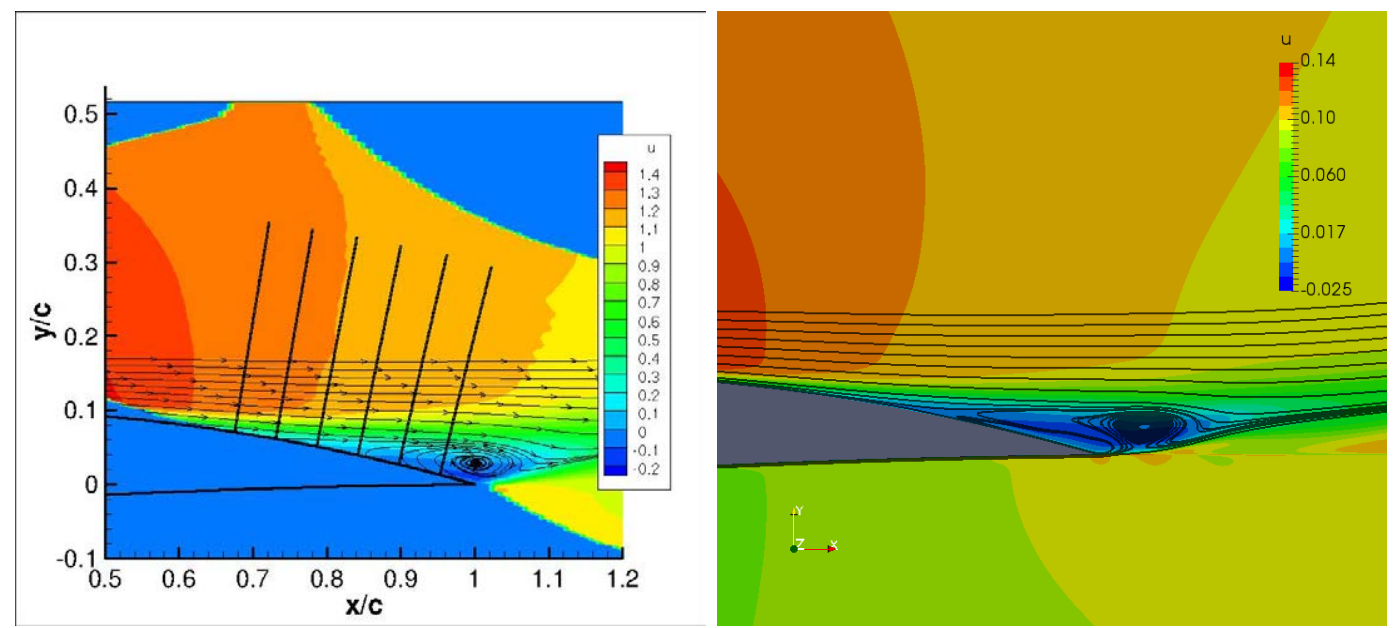

Figure D.12: 2DN44 Experimental (left) and CFD generated (rigth) velocity map and streamlines 


\section{D.4 2D NACA 0012 airfoil (2DN00)}

The 2-D NACA 0012 airfoil validation case involves the flow over a NACA 0012 airfoil (symmetric, $12 \%$ thickness) at several angles of attack. Balance and pressure data are available from multiple data sources, but NASA turbulence models resource recommends the comparison against the Gregory and O'Reilly data (Gregory and O'reilly, 1973). The recommendation from the same resource is to run the cases at $M=0.15$ and $R e_{c}$ equal to 6 million. Figure D.4 shows the domain geometry and the utilized boundary conditions. The NASA provided CGNS unstructured quad grid has been used for the $2 \times 449 \times 129$ version (2x257 points on the airfoil surface). A detail of the mesh can be seen in figure D.14. It is the third to finest mesh (and coarsest), with a minimum

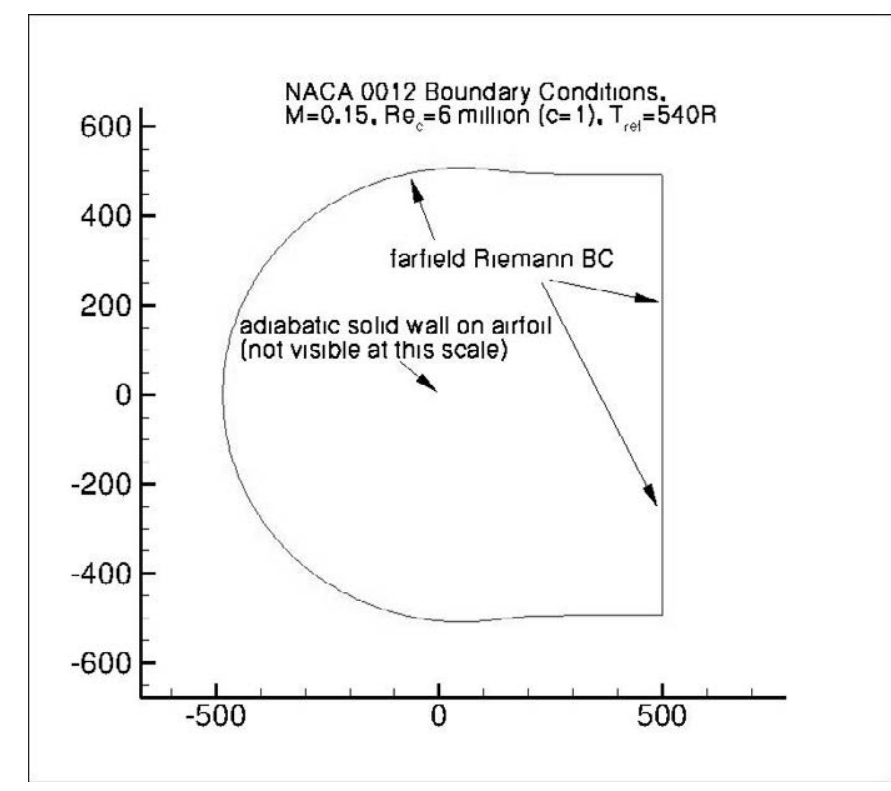

Figure D.13: 2DN00 Boundary conditions
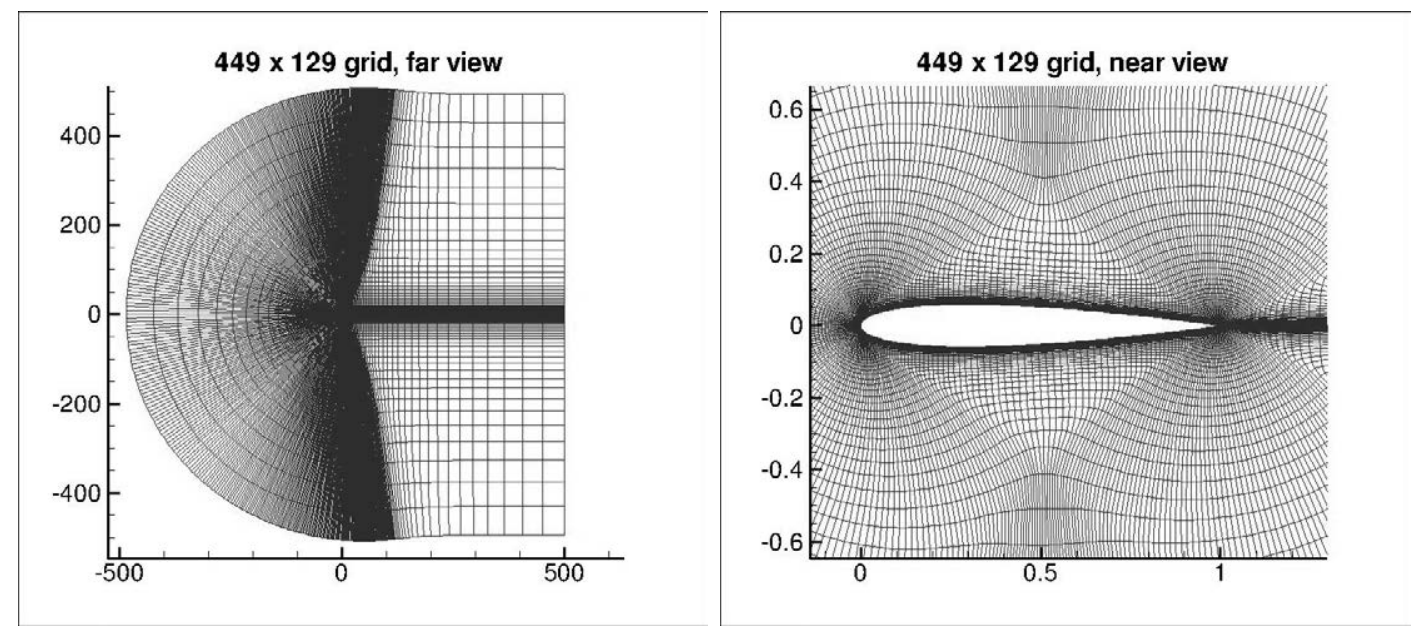

Figure D.14: 2DN00 Mesh detail 
spacing at the wall of $y=1.6 \cdot 10^{-6}$, resulting in an approximate average $y^{+}$equal to 0.8. The CFD code has been run with fully turbulent inflow conditions with $\tilde{\nu}=3.0$.

Figure D.16 shows the comparison between the experimental data (for the upper surface only) and the CFD generated data. As it can be seen, the agreement is excellent for the three different angles of attack.

Also of interest is the comparison with the experimental overall airfoil lift. The obtained lift values, superimposed on top of NASA resource provided figure, can be seen in figure D.15. The agreement is very good, although it slightly over estimates the lift before the stall occurs.

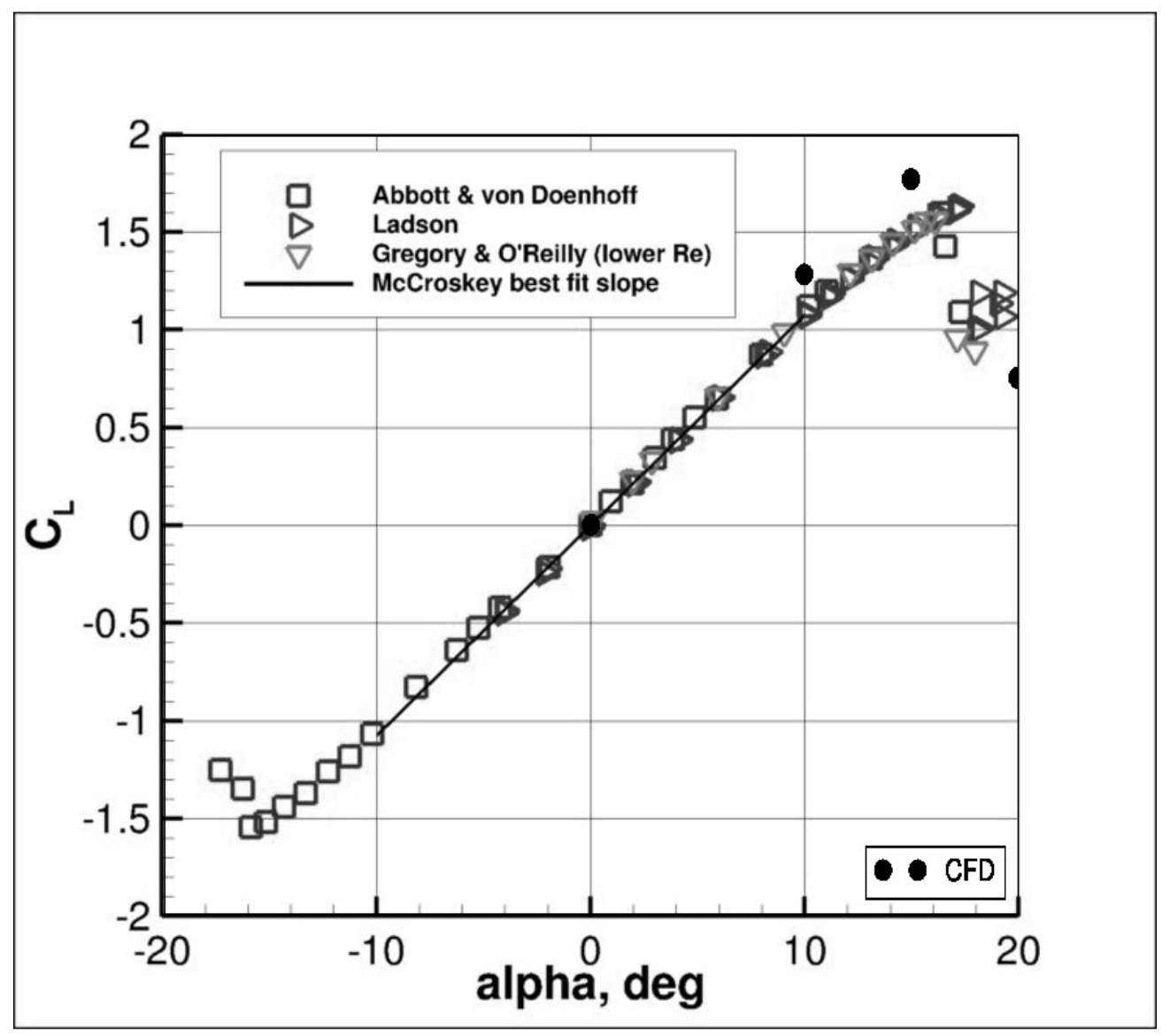

Figure D.15: 2DN00 Obtained $C_{L}$ superimposed on top of available experimental data 

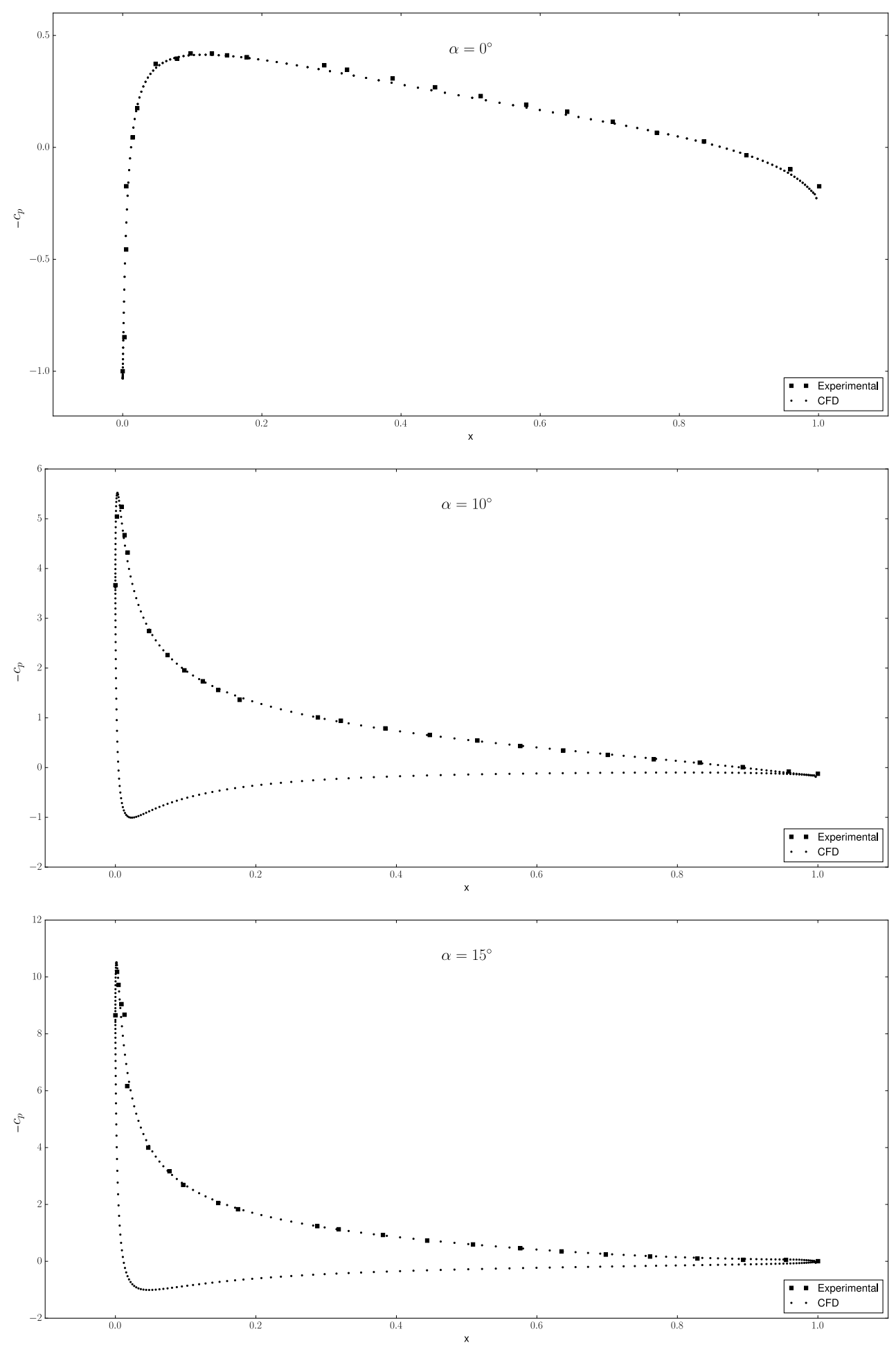

Figure D.16: Experimental (upper surface) and CFD generated pressure distributions over the airfoil for $\alpha=0^{\circ}$ (top), $\alpha=10^{\circ}$ (middle) and $\alpha=15^{\circ}$ (bottom) 


\section{D.5 2D NACA 0012 Transonic comparison with experiment}

The same mesh as in the previous section has been run at transonic conditions for $\mathrm{M}=0.756$ at $R e_{c}$ equal to 4 million at $\alpha=0^{\circ}$ and $\alpha=1.95^{\circ}$. The pressure distribution is compared against available experimental data (Cook et al., 1979, p. A1-9, A1-13).

The comparison between the available experimental pressure data over the airfoil and the obtained by the CFD can be seen in figure D.17. The agreement is overall very good in terms of pressure distribution, with some small discrepancies in the location of the shock. The CFD overpredicts slightly the presence of the shock in the case of $\alpha=0^{\circ}$. In the case with $\alpha=1.95^{\circ}$ the shock position is predicted to be slightly further aft $(\approx 6 \%)$ when compared to the experimental data. Note that no refinement has been performed in the mesh in order to capture the shock adequately.
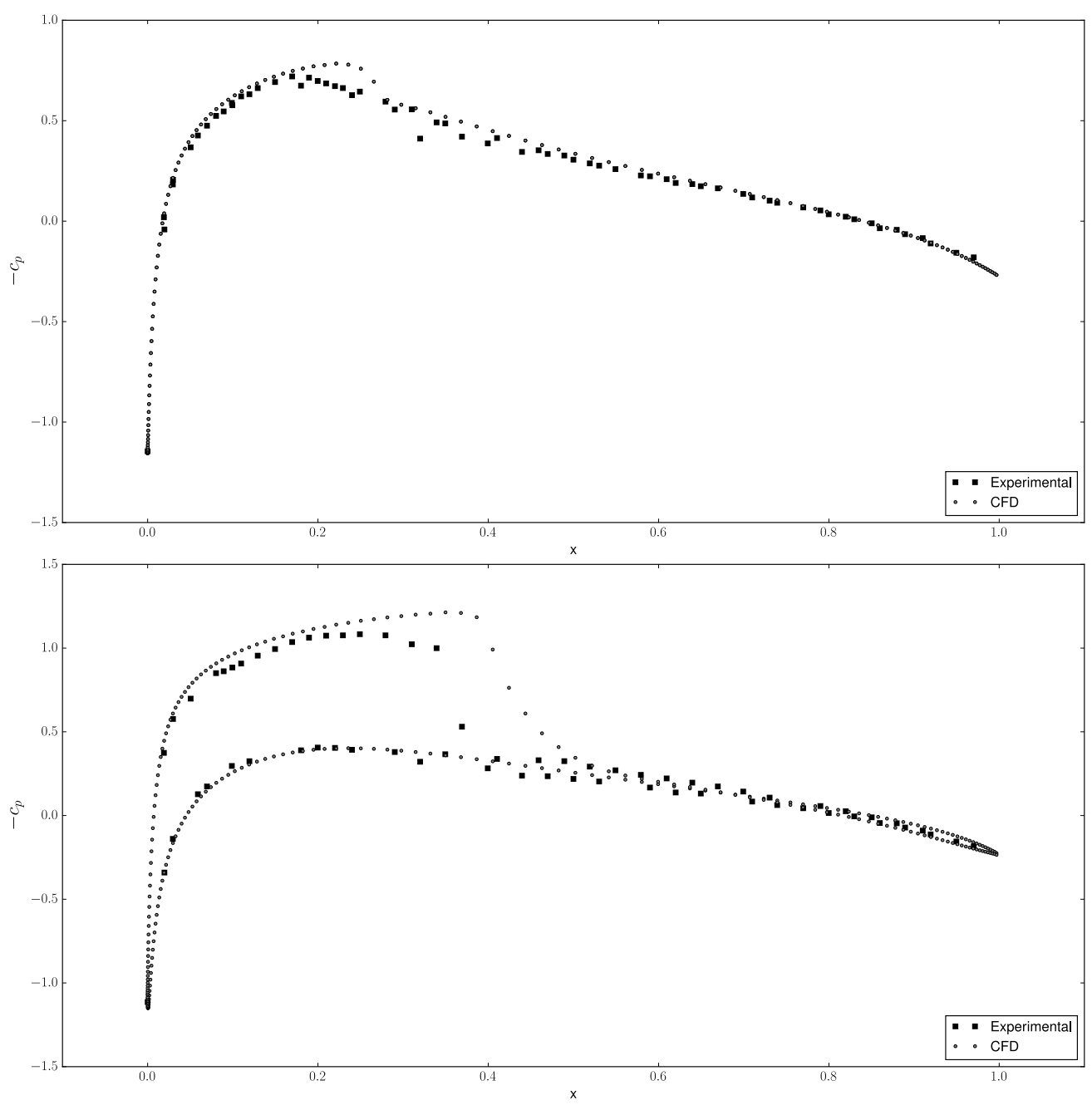

Figure D.17: NACA0012 at $\mathrm{M}=0.756$, pressure data comparison between CFD and experimental data for $\alpha=0^{\circ}$ (top) and $\alpha=1.95^{\circ}$ (bottom) 


\section{D.6 2D NACA0012 forced oscillation comparison with in- viscid simulations}

An extra validation step has been taken with the comparison between the inviscid and turbulent viscous simulations. Figure D.18 shows a comparison between the resulting lifts for a forced angle of attack variation of the form $\alpha=a(\cos (2 \pi f t)-1)$, with $a=0.01 \mathrm{rad}$ and $f=10 \mathrm{~Hz}$, at $\mathrm{M}=0.30$ and $\mathrm{M}=0.80$.

For $\mathrm{M}=0.30$ there is a delay of the lift for the viscous case, with respect to the inviscid case, of approximately $8^{\circ}$. The viscous simulations result in approximately $7 \%$ higher forces.

For $\mathrm{M}=0.80$ there is a delay of the lift for the inviscid case, with respect to the viscous case, of approximately $5^{\circ}$. The viscous simulations result also in an approximately $5 \%$ higher forces.

Both simulations give comparable results, given the fact that the runs are performed in equivalent, but different, numerical meshes.
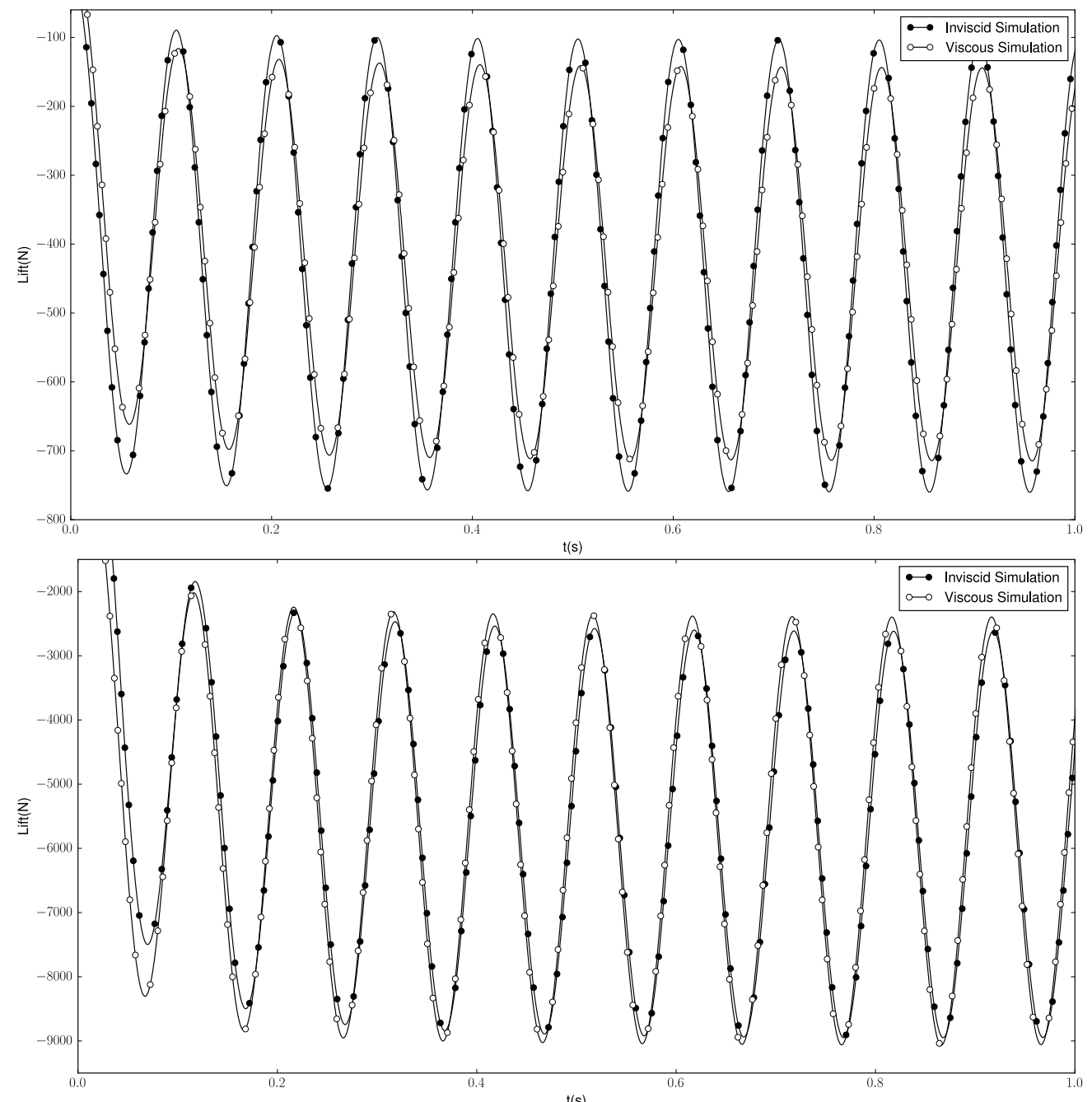

Figure D.18: 2D NACA0012. Lift comparison between inviscid and viscous turbulent simulations at $\mathrm{M}=0.30$ (top) and $\mathrm{M}=0.80$ (bottom) 